\title{
Charakterisierung von einer retrograden Modulation synaptischer Transmission im Kleinhirn der Ratte
}

\author{
Dissertation \\ zur Erlangung des Doktorgrades \\ der Mathematisch-Naturwissentschaftlichen Fakultäten \\ der Georg-August-Universität Göttingen
}

vorgelegt von

Marco Alberto Diana aus Mailand

Göttingen, den 31. Januar 2003 
D7

Referent: Prof. Erwin Neher

Koreferent: Prof. Theo Geisel

Tag der mündlichen Prüfung: 31. Januar 2003 


Sono un povero stupido so solo che Chi e' stato e' stato e chi e' stato non e' Chi c'e' c'e' e chi non c'e' non c'e' Consumati gli anni miei, vistosi movimenti sulla Terra, grandiosi necessari, futili patetici Come fare non fare, quando dove perche' E ricordando che tutto va come va Come fare non fare, quando dove perche' E ricordando che tutto va come va

A tratti, Giovanni Lindo Ferretti, C.S.I. 



\section{Acknowledgments}

There are many people who have accompanied me through these years: some continuously, some only for short but important periods. There were some who were close to me from Italy, others in Goettingen, others now in Paris, there are a few who have bridged periods and countries and who are still there.

First of all, the lab: Alain Marty and Isabel Llano have given me the chance to work with them, to explore (sometimes crazy) hypotheses with much freedom and many means while, at the same time, trying to correct my focus when it was pointing in too many directions.

Of course, I want to thank Prof. Neher, my official $\mathrm{PhD}$ supervisor and a constant point of reference at the MPI in Göttingen.

In Italy, my small family followed my wanderings always with warmth and support: my mother, my sister Michela and Mino (who constantly and endlessy fed me during my frequent stays in Milan), la gatta Marta, la mia Chiara and Lia, my quasi-sister from the Dibit time; then Giorgio, Lisa e il suo fidanzato nonche' mio migliore amico Federico, IlariaPoldaMaria, Alessandra.

The bunch in Goettingen: my dear friend Julieto, Box, Uta, Dario, Lars, Ralf, Dieter and the squash boys, Olivier and Elena and, especially, Roberta. Thank you Sigrid for the help with the immunocytochemistry.

Then, thank you Sandra.

In Paris: Carolissima, Philippe and Jacsue "les Aschers", Rossella, Thibault, Ivan Ivanovich, Elena, Marco and Manuela and, how could I not mention them?, Tonino, Lazare and, finally, Gnasu.

Now, those stronger than borders: Micaela, thanks Christophe for everything, Vera, Joel, Paola and Martin, Celine, Prof. Dr. Pilot Yusuf, Kevin and Alessio.

I am deeply indebted, in space and time, to Miss Alligalli.....

Finally, there were emotions from other sources, just one for all: Giovanni Lindo Ferretti e i suoi CSI, who were (sometimes too) often in my ears. 



\section{Summary}

In recent years, a lot of interest has been given to retrograde modulatory mechanisms of synaptic transmission: in many regions of the central nervous system, substances released from the postsynaptic cell are indeed able to control afferent inputs through the activation of presynaptic pathways.

In this thesis, I present data concerning one such phenomenon called Depolarization-induced Suppression of Inhibition (DSI). DSI consists in a short-term form of inhibition of GABAergic transmission from basket/stellate interneurons in the molecular layer of the cerebellum onto Purkinje cells. The calcium entry following a depolarization of the postsynaptic cell leads to a fully reversible decrease of the afferent inhibitory input, which recovers in little more than 1 minute at room temperature. DSI was studied in sagittal slices from the cerebella of juvenile rats, using electrophysiological, calcium imaging and morphological techniques, as follows:

* The DSI of action potential-dependent and independent GABAergic currents was characterised by recording spontaneous synaptic activity from single Purkinje cells.

* These data were complemented by performing paired recordings from presynaptic interneurons and postsynaptic Purkinje cells. The perforated patch technique was used to record from and stimulate presynaptic interneurons: this prevented the irreversible rundown of synaptic currents given by the presynaptic whole-cell configuration.

* DSI was unequivocably shown to consist in a presynaptic reduction of synaptic transmission. The study of several synaptic parameters in paired recordings confirmed and expanded previous experimental data suggesting a presynaptic site of origin of DSI.

* The nature of the retrograde messenger(s) was identified: postsynaptically-produced endogenous cannabinoids activate presynaptic type 1 cannabinoid receptors (CB1Rs) to in- 
hibit transmission during DSI.

* Immunohistochemical techniques confirmed the presence of the receptors on presynaptic structures in the molecular layer.

* Imaging experiments of calcium transients in interneuron axons showed that CB1Rs exert their inhibitory action at least by reducing the amount of calcium entry per action potential into presynaptic terminals.

* To account for the total decrease of transmission, a quantitative evaluation of all the components of DSI was done. This predicted that DSI must involve a further, so far not described, form of inhibition. Indeed, by depolarizing Purkinje cells, an early excitatory and a late inhibitory modulation of the spontaneous firing frequency of interneurons was found in paired recordings. These phenomena were characterized; the late, inhibitory one was shown to depend on the activation of presynaptic CB1Rs and to give an important quantitative contribution to DSI (accounting for $23 \%$ of the overall inhibition of the spontaneous GABAergic input onto Purkinje cells).

* Interneurons were shown to form an extensive network of electrically connected cells; the widespread inhibitory effect on firing during DSI was evidenced through its action on gap junctional spikelets.

All these different facets of DSI were discussed and possible molecular mechanisms mediating them were proposed. Particular attention was given to the characterization of the powerful modulatory action of the cannabinoid system on GABAergic synaptic transmission. 


\section{Contents}

\section{$\underline{\text { Acknowledgments }}$}

\section{Summary}

1 Cerebellar Purkinje cells and their inhibition $\underline{15}$

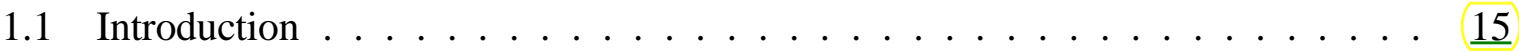

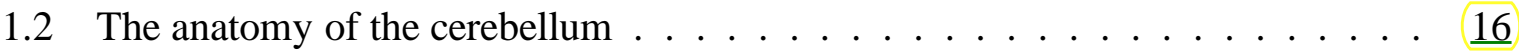

1.3 Cell types and synaptic connections . . . . . . . . . . . 18

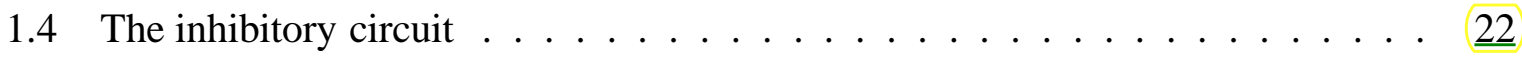

1.5 The basket/stellate cell to Purkinje cell synapse: a closer look . . . . . . . . $\underline{25}$

1.5.1 The basket/stellate cell to Purkinje cell synapse in a developmental perspective .................... . . 26

1.6 A few words on electrical synapses . . . . . . . . . . . . 28

2 Synapses and Retrograde Messengers $\underline{\mathbf{3 1}}$

2.1 Depolarization-induced Suppression of Inhibition (DSI) . . . . . . . . . 32

2.1 .1 Calcium dependence of DSI . . . . . . . . . . . . 33

2.1.2 DSI is expressed presynaptically? ............ $\underline{35}$

2.1.3 Which retrograde messenger(s) for DSI $\ldots \ldots \ldots . \ldots 37$

2.1.4 Action Potential propagation failures as (one of many) possible mechanisms for DSI . . . . . . . . . . . . . . . . . . . . . . . 39

2.1.5 Unresolved Questions . . . . . . . . . . . . . $\underline{40}$

3 The Cannabinoid System $\underline{41}$

3.1 Cannabinoids and their receptors $\ldots \ldots \ldots \ldots \ldots$ 
3.1.1 Type $1(\mathrm{CB} 1 \mathrm{R})$ and Type $2(\mathrm{CB} 2 \mathrm{R})$ cannabinoid receptors . . . . . $4 \underline{41}$

3.1.2 What about a CB3 receptor... . . . . . . . . . . 45

3.1.3 ...and actions through other receptors? . . . . . . . . $4 \underline{45}$

3.2 Endogenous cannabinoids: identity, metabolism and uptake . . . . . . . . $\underline{46}$

3.2 .1 Identity and metabolism . . . . . . . . . . . . . .

3.2 Uptake .........................

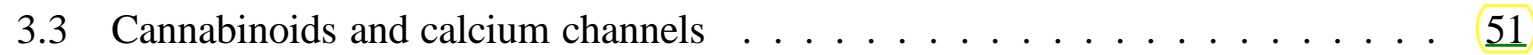

3.4 Cannabinoids and potassium channels . . . . . . . . . . . $\underline{52}$

3.4.1 Influence of presynaptic $K^{+}$channels on synaptic strength . . . . . $\underline{52}$

3.4.2 Reported effects of CB1R activation on potassium conductances . . . $\underline{54}$

3.5 Other possible targets . . . . . . . . . . . . . . $\underline{55}$

4 Materials and Methods $\quad \underline{59}$

4.1 Preparation for electrophysiology . . . . . . . . . . . . . . 59

4.2 Electrophysiological recordings ............... . . $\underline{60}$

4.3 Electrophysiological data analysis . . . . . . . . . . . . . . 62

4.3.1 Analysis of the DSI of mIPSCs, of sIPSCs and of interneuron action potentials ...................... 62

4.3.2 Analysis of the DSI of eIPSCs . . . . . . . . . . . $6 \underline{63}$

4.4 Fluorometric calcium imaging experiments and their analysis . . . . . . . $\underline{65}$

4.5 Morphological reconstructions . . . . . . . . . . . . . 66

4.6 Immunohistochemistry . . . . . . . . . . . . . . . . 67

5 Results: DSI characterization $\underline{69}$

5.1 DSI of spontaneous TTX-sensitive and of miniature TTX-insensitive IPSCs . . $\underline{69}$

5.2 DSI as measured in paired recordings . . . . . . . . . . . . . 71

5.2.1 DSI as measured with presynaptic perforated patch recording: why the perforated patch and not the whole-cell configuration? . . . . . $\underline{72}$

5.2.2 Purkinje cell depolarizations induce DSI of eIPSCs . . . . . . . $\underline{75}$

5.2.3 Reliability of induction and time course of DSI . . . . . . . . . . 79

5.2.4 DSI induction is not dependent on the intracellular solution of the post-

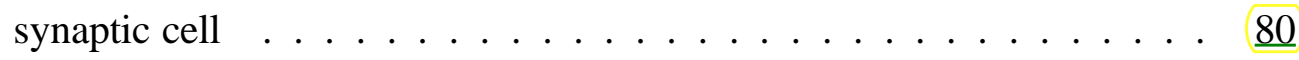

5.2 .5 Increase in the failure rate during DSI . . . . . . . . . . $\underline{82}$ 
5.2.6 Paired Pulse Ratio (PPR) and DSI . . . . . . . . . . . . . $\underline{84}$

5.2.7 Kinetic parameters of eIPSCs and DSI . . . . . . . . . $\underline{86}$

5.2 .8 Coefficient of variation and DSI . . . . . . . . . . $\underline{88}$

6 Results: DSI and cannabinoids 91

6.1 Endocannabinoids are the retrograde messengers mediating DSI . . . . . . 91

6.1.1 Localization of CB1Rs in the cerebellum of juvenile rats . . . . . . . 91

6.1.2 Implication of CB1Rs in DSI: paired recording experiments . . . . 93

6.1.3 Effect of CB1R activation and inhibition on mIPSCs . . . . . . . . 97

6.1.4 Effect of CB1R activation and inhibition on DSI, as measured in miniature recording experiments . . . . . . . . . . . . . . . 99

6.1.5 Effect of WIN 55, 212-2 on presynaptic calcium transients . . . . . . . 99

6.2 DSI, CB1R activation and interneuron firing frequency . . . . . . . . . 101

6.2.1 Purkinje cell depolarizations inhibit firing in basket/stellate cells . . . . 101

6.2.2 DSI-inducing protocols increase the threshold for action potential triggering in basket/stellate cells . . . . . . . . . . . . . . . . 106

6.2.3 DSI-induced inhibition of gap junctional spikelets in basket/stellate cells $\underline{108}$

6.2.4 The inhibition of interneuron firing is mediated by endogenous cannabinoids . . . . . . . . . . . . . . . . . . . . 114

6.2.5 CB1R activation by WIN 55, 212-2 inhibits interneuron firing . . . . 116

6.2.6 What mechanisms for the CB1R-induced inhibition of firing? . . . . 117

6.2.7 The increase in firing rate during Purkinje cell depolarization: a "true" or an artefactual effect? . . . . . . . . . . . . . . . . . . 120

7 DISCUSSION

7.1 The various components of DSI: a quantitative evaluation . . . . . . . . . 123

7.1.1 Further arguments for the existence of multiple mechanisms underlying DSI . . . . . . . . . . . . . . . . . . . 128

7.2 Paired Pulse Ratio: comparison with previous work . . . . . . . . . . . . 129

7.3 Increased failure rate during DSI . . . . . . . . . . . . . . . . 131

7.4 An endocannabinoid as retrograde messenger for cerebellar DSI . . . . . . 131

7.5 What about the glutamate hypothesis? . . . . . . . . . . 133

7.5.1 Glutamate, group 1 mGlu receptors and endocannabinoids . . . . . . 134 
7.6 Controlling presynaptic spike rate: a novel form of plasticity $\ldots \ldots \ldots \underline{136}$

7.6.1 The "early", excitatory effect . . . . . . . . . . . . . 137

7.6.2 The "late", inhibitory effect . . . . . . . . . . 138

7.6.3 A comparison with the hippocampus . . . . . . . . . . . 140

7.7 Cannabinoids and gap junctions $\ldots \ldots \ldots \ldots \ldots \ldots$

Bibliography $\quad \underline{143}$

Lebenslauf $\quad \underline{168}$ 


\section{Chapter 1}

\section{Cerebellar Purkinje cells and their}

\section{inhibition}

\subsection{Introduction}

As indicated by its name (in latin cerebellum means small cerebrum, with the latter word denoting the brain), the cerebellum is a well separated and distinct part of the CNS, located dorsally with respect to the main cerebral structure (see Fig.3.1).

Although it is a very ancient part of the vertebrate brain and its dimensions vary enormously between animal species, its cellular and circuital organization has remained remarkably constant along the evolutive tree, this fact most likely indicating the universality of its role and functions. The cerebellum, for its peculiar structure, has been for many decades (for some centuries now, it would also be appropriate to say) "THE" model to study for morphologists. We have detailed descriptions of its organization, which run back to the first years of the nineteenth century (just notice, for example, that Purkinje, who gave his name to the biggest cerebellar cell type, described the molecular layer in 1838 or that Ramon y Cajal started his career as CNS morphologist in 1886, mainly concentrating on the cerebellum).

As far as research on basic synaptic mechanisms is concerned, the features of its structure are extremely favourable; we will see, for example, that the morphology of the molecular layer and of the there lying axons of GABAergic interneurons makes the task of finding synaptically connected cell pairs incomparably easier with respect to other central areas like the neocortex, the hippocampus or the retina.

Since the aim of this thesis has been to characterize a synaptic phenomenon, which is at high 
probability a property common to many different central connections, I will not describe the different models, which try to specify the macroscopic functions of the cerebellum in the context of the overall functionality of the CNS; I will limit myself to depict the general structure of the rodent cerebellum, especially highlighting the properties giving advantages to the experimental work I have performed, and the possible topics related to its postnatal development, which could have influenced it.

\subsection{The anatomy of the cerebellum}

Unique structure of the central nervous system straddling the midline without interruption, the mammalian cerebellum can be divided into three main areas: a central vermis (which constitutes almost all of the cerebellum in lower vertebrates) and two simmetrical lateral hemispheres, whose dimensions are correlated with the position on the evolutive tree of the animal species considered.

At a bigger magnification, the general cellular and morphological organization of the cerebellum remains constant throughout these divisions and can be described by first examining it more closely in sagittal sections (see Fig. 1.1): one characteristics appearing at the very first look is that the cerebellar cortex is an extremely folded structure: the several invaginations of its cortex (called lobules) allow a great total surface to be packed in a relatively small region (just think that the human cerebellum covers a total area of 50,000 squared centimeters - on average 1000 $\mathrm{mm}$ wide and $50 \mathrm{~mm}$ wide - in a $6 \mathrm{~cm} \times 5 \mathrm{~cm} \times 10 \mathrm{~cm}$ volume). Although morphologists are still discussing about a universal classification of cerebellar foldings, it can be stated that the rodent brain has four main lobes (along the rostro caudal axis: the anterior, the central, the posterior and the inferior lobes; sometimes central and posterior lobes are altogether defined as posterior lobe while the inferior lobe is also commonly called flocculonodular lobe), themselves further subdivided into ten smaller lobules. it is worth remarking that the lobules run parallel to the transversal direction: a sagittal section anywhere along the medio-lateral axis, thus, reproduces basically the same gross (and, we will see, even microscopic) structure and the whole cerebellum could be imagined as given by the alignment along the transversal axis of consecutive sagittal slices.

The cerebellar cortex is divided into well defined layers; from the external pial surface to the 


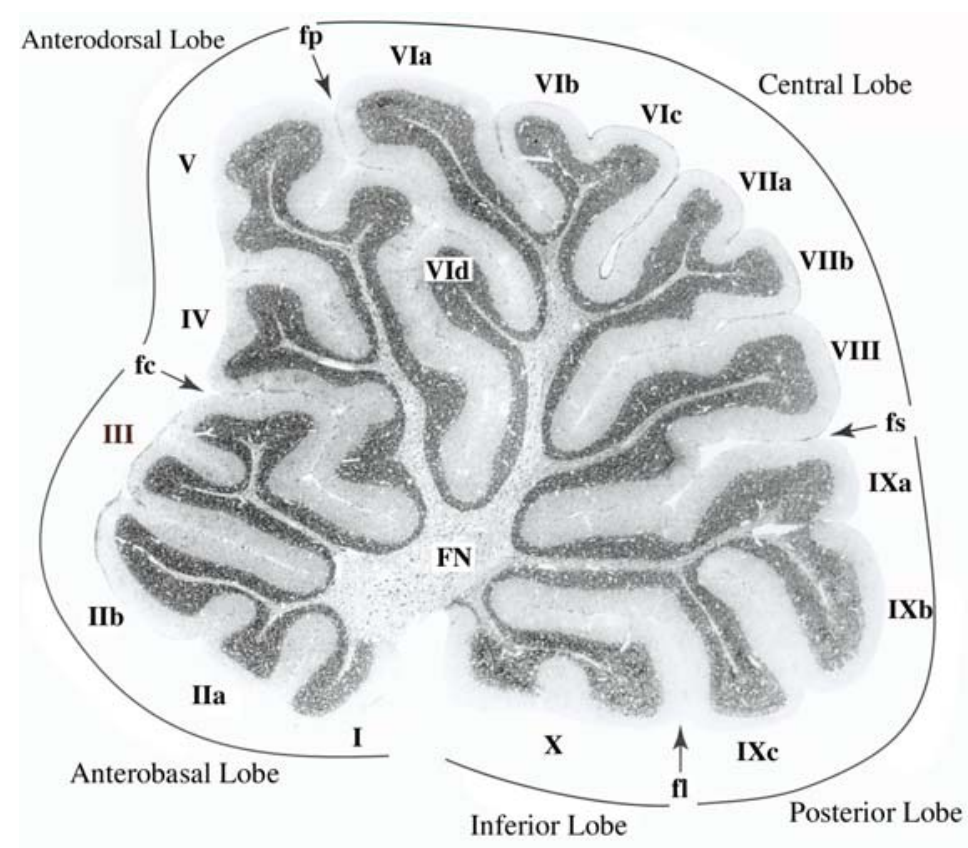

Figure 1.1: The rat cerebellum as seen from sagittal sections. The boundaries of the four different main lobes can be observed; the anterior lobe has been further subdivided by these authors (from Altman and Bayer, 1997) into 2 subdivisions. The 10 smaller lobules are indicated by latin numbers. Notice that this image exactly represents the experimental preparation used in this thesis $(\mathrm{fc}=$ fissura preculminata; $\mathrm{fl}=$ fissura posterolateralis; $\mathbf{f p}=$ fissura prima; $\mathbf{f s}=$ fissura secunda; $\mathrm{FN}=$ fastigial nucleus). 
internal white matter, three layers can be distinguished: the molecular layer, the Purkinje cell layer and, finally, the granule cell layer (see Fig.6.1).

As suggested by its name, the granule cell layer contains the somata of the granule cells and those, fewer in number, of inhibitory neurons like Golgi and Lugaro cells. The Purkinje cell layer is composed of a monolayer of Purkinje cell soma, running parallel to the pial surface and, thus, to the lobular foldings, and by Bergmann glial cell somata, which are closely juxtapposed to the soma of the Purkinje cells; finally, the molecular layer contains the parallel fibers (the ascending/ed axons of the granule cells), the enormous arborizations of Purkinje cell dendrites, other glial cell somata and the so-called stellate and basket cells, which are the GABAergic interneurons whose synapses onto Purkinje cells are the object of my studies.

Besides the modularity and, thus, the great homogeneity of the cerebellum, another striking and extremely useful property is its geometrical regularity: Purkinje cell dendrites lie planarly in the sagittal plane containing the soma from which they originate (the same plane of Fig.1.1 and Fig.1.2), which also contains the Bergmann cell neurites extending up to the pial surface, the axons of basket and stellate cells and their bifurcations and collaterals, the climbing fibers synapsing onto the Purkinje cells and, even if with more lateral spread, the axons of Purkinje cells. On the contrary, the parallel fibers, after leaving their originary granule cell soma, climb up the granule cell layer to split with a "T" junction in the molecular layer and then continue their course through consecutive Purkinje cell dendritic arborizations (where they synapse) in the transversal plane.

In the following Fig.1.2 and Fig.1.3, this ortogonal, planar organization of the cerebellum can be appreciated; both illustrations are from the 1995's re-edition of Ramon y Cajal's 1911 "Histologie du systeme nerveux de l'homme et des vertebres". Ramon y Cajal has given us descriptions of the cerebellar cortex, which are still today unrivalled for precision, detail and beauty.

\subsection{Cell types and synaptic connections}

Purkinje cells are the only output of the cerebellum. Their axon exits the lower part of the soma at the edge of the granular cell layer, continues its way downwards while sending off collaterals, which ascend again to form a plexus at the level of the Purkinje cell layer and of their first dendritic arborizations, where they form synapses. The main axonal shaft reaches the deep 


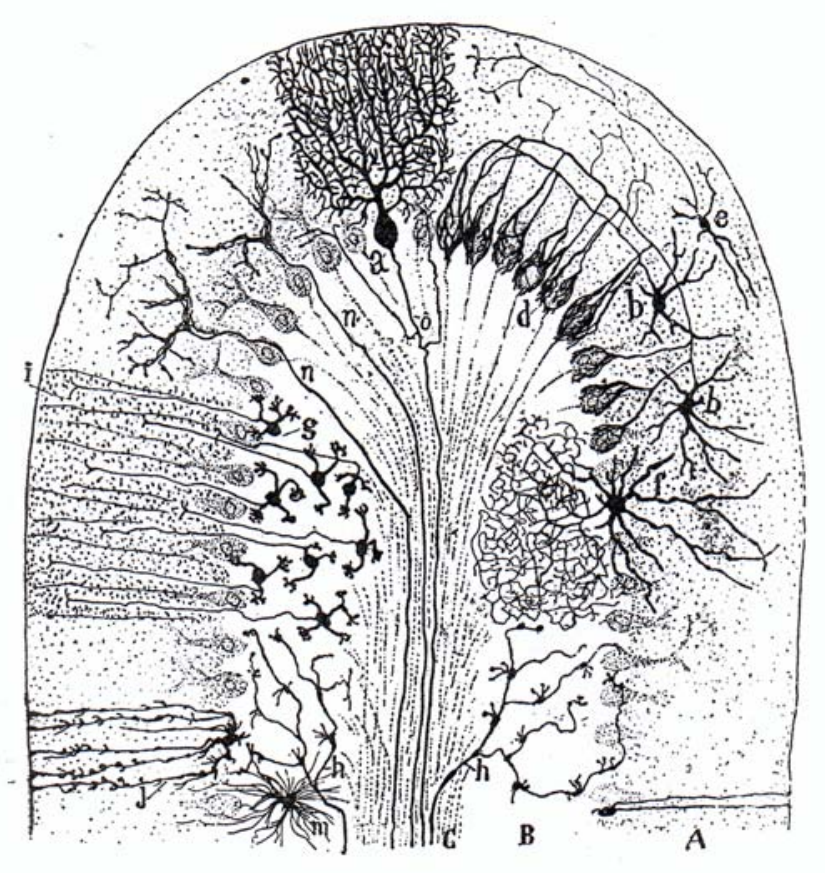

Figure 1.2: Microscopic structure of the cerebellum in the sagittal plane. This picture, taken from Ramon y Cajal, 1995, shows in detail the cellular types and the distribution of their fibers in the sagittal cerebellum; one lobule is illustrated. Purkinje cells form a one cell thick layer at the border between the molecular layer (ML; indicated by A) and the granular cell layer (GL; indicated by B); notice the extensive dendritic tree of these cells, which extends up to the more external border of the section (the only fully depicted Purkinje cell is indicated by the letter a in the upper part of the reproduction). Some basket (b) and stellate (e) cells are shown in the ML on the right side of the section; their axons run parallel to the Purkinje cell layer. Granule (g) and Golgi cells (f) cells are also portrayed, as well as afferent mossy (h) and climbing (n) fibers. This picture represents the cellular structure present in the preparation, which has been used for the experiments of this dissertation. 


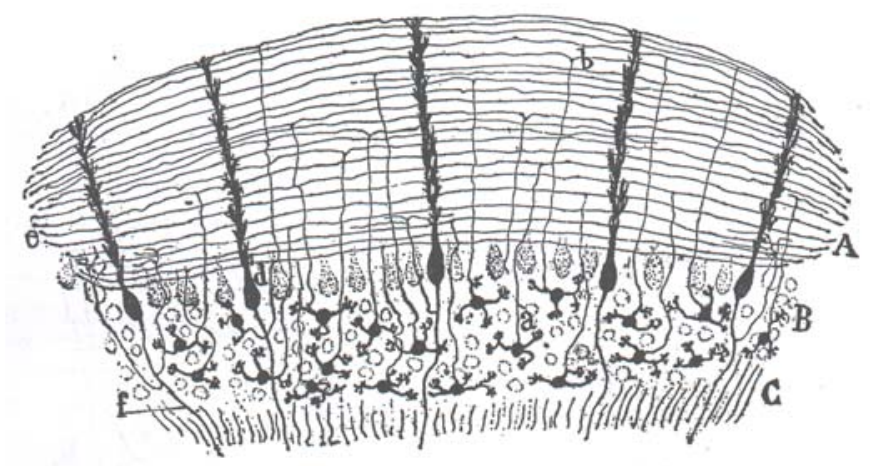

Figure 1.3: Here, Ramon y Cajal depicts the cerebellar structure along a transversal plane. In this direction, parallel fibers (the axons of the granule cells) run up from to the GL (B) to the ML (A), where they divide (b) and continue their course perpendicularly through consecutive Purkinje dendritic trees, where they form synapses.

cerebellar nuclei where it forms GABAergic synapses onto the principal cells of this area: thus, the cerebellar output is inhibitory.

There are two independent systems bringing information into the cerebellum: the first one is given by the so called climbing fibers, myelinated axons originating from the neurons of the inferior olive in the brainstem: after losing their myelination in the granular cell layer, they crawl and climb over the Purkinje cell dendritic arborizations forming up to several hundreds functional glutamatergic synapses onto it (see Fig.1.4); it should moreover be remarked that each Purkinje cell receives input only from one climbing fiber, in a process of input selection strictly controlled during development. The second input comes from the mossy fibers, which, in contrast to the climbing fibers, originate from many distinct CNS regions: for example, the vestibular nerve and nuclei, the spinal cord, the reticular formation and the cortex. They enter the cerebellum through the cerebellar peduncles and, similarly to the climbing fibers, they send off collaterals to the cerebellar deep nuclei before branching and ending up synapsing in the granule cell layer onto granule cell dendrites. These synapses are glutamatergic, thus excitatory.

This second, external excitatory input to the cerebellum is transmitted to Purkinje cells by the parallel fibers after a first integrative step in the granular cell layer.

The parallel fibers, as explained, run along the transversal axis of the molecular layer for several hundred microns, forming glutamatergic synapses both on stellate, basket and Golgi cell dendrites and on Purkinje cell dendritic spiny branchlets (on the contrary, climbing fiber terminals 


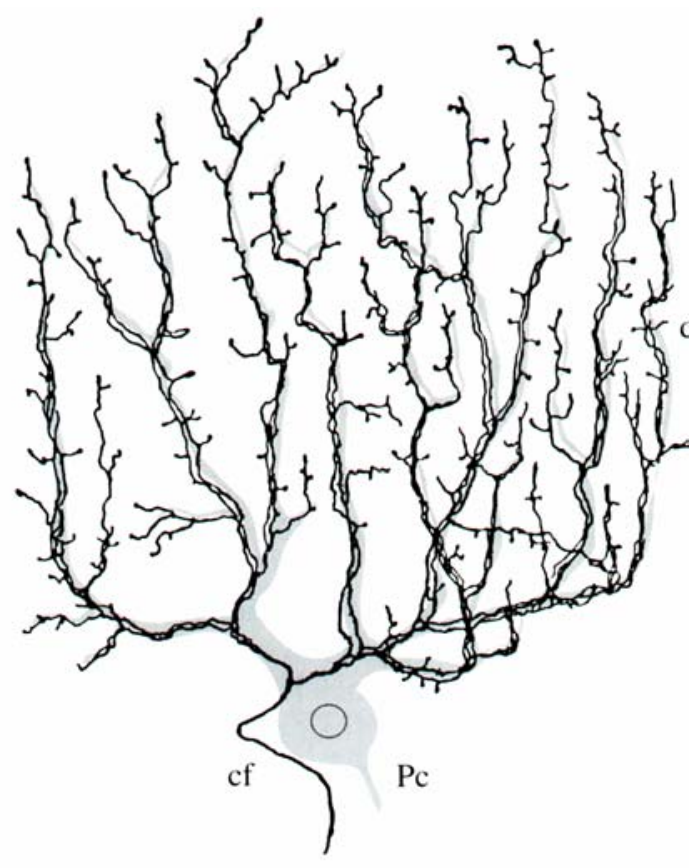

Figure 1.4: A Purkinje cell (Pc, light grey) receives inputs from a single climbing fiber (cf): in this illustration (by Ramon y Cajal, 1911 and reproduced in Altman and Bayer, 1997) a climbing fiber can be seen crawling along the dendritic arborizations of a Purkinje cell, where it forms multiple glutamatergic synapses. This synaptic input from climbing fibers is one of the most powerful excitatory drive onto a single cell in the whole central nervous system; typically, Purkinje cells react to this stimulation with the so called "complex spike", a prolonged and strong depolarization mediated in large part by calcium conductances (see Shepherd, 2001). 

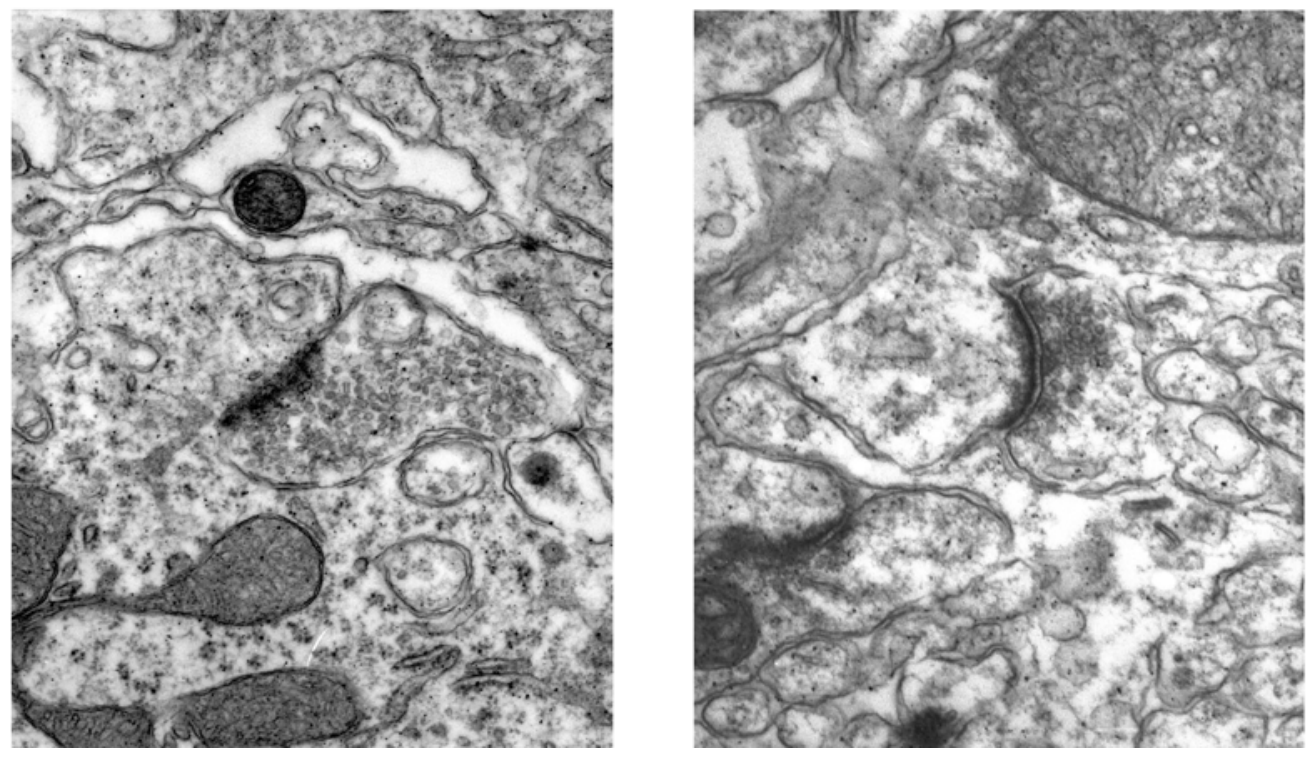

Figure 1.5: EM images of parallel fiber terminals onto dendritic spines of a juvenile (12 days old) Purkinje cell. The asymmetrical distribution of electron dense material (notice the darker staining of the postsynaptic membrane with respect to the presynaptic one) and the presence of small, round vesicles identify the connection as an excitatory synapse; they are most likely formed by parallel fibers and not by climbing fibers for the presence of a postsynaptic spine-like structure and for the fact that climbing fiber terminals most often form several active zones per area of connection, with no specific presynaptic bouton but, rather, a widespread and sparse terminal area.

occur on the smooth dendritic body or on bigger protuberances bulging from the main dendritic shafts of the Purkinje cells).

In Fig.1.6 two such connections can be seen at EM level; I have taken these images from a 12 days old rat. Adult Purkinje cells receive an enormous number of such contacts, from 150,000 to 175,000 ; this is the highest number of synaptic inputs a single neuron receives in the whole CNS.

\subsection{The inhibitory circuit}

The excitatory input to Purkinje cells is modulated by the inhibitory circuit present in the cerebellum; there are four main cellular types that exert an inhibitory action: GABAergic basket and stellate cells, whose cell bodies lie in the molecular layer, GABAergic Golgi cells and mixed 
GABA/Glycinergic Lugaro cells (Dumoulin et al., 2001), whose cell bodies are located in the granular cell layer.

In this thesis, I have studied the synaptic connections between basket/stellate cells and Purkinje cells. Even though basket and stellate cells are always dealt with in the literature on the cerebellum as different cell types, actually no physiological criteria or molecular marker have been found, which can distinguish them in terms of functionality. The distinction between the two types is given only by the position of their somata in the molecular layer: GABAergic interneurons placed in the lower third of the molecular layer are defined as basket cells, interneurons in the outer two-thirds are stellate cells.

There is a continuous change in morphological features while moving towards the external pial surface; low basket cells have normally bigger bodies, a few, thick dendrites which can spread throughout the molecular layer to reach the outer limits of the cerebellar cortex and an axon extending up to several hundred microns in the sagittal direction parallel to the Purkinje cell layer.

The main axonal shaft makes synaptic terminals onto Purkinje cell and other interneuron dendrites and soma, while also sending off collaterals at right angles in both directions: while ascending branches are normally thin and short, descending ones reach the Purkinje cell layer and create further arborizations, which wrap up in a basket-like structure around the Purkinje cell bodies and, finally, around the initial section of the Purkinje axon.

A peculiarity of these "low" interneurons is that collaterals from many of these cells (even more than 20 in adult animals) are packed together around the Purkinje axon hillock, with surprisingly few axo-axonic synapses there (see Somogyi and Hamori, 1976): the so-called pinceau is, thus, most likely a mainly electrical shunting device, able to inhibit the Purkinje cell output only by electrical field interactions (Korn and Axelrad, 1980), as seen for example in the goldfish Mauthner cell (Korn et al., 1978; see how pinceaux normally appear in light microscopy in Fig.3.2).

The most external stellate cells are normally smaller in dimensions (in the most superficial regions of the molecular layer, stellate cells can have even only from 5 to $9 \mu$ m-diameter somata), with shorter dendrites extending mainly sagittally in the molecular layer and shorter axons, which synapse on the distal dendritic arborizations of Purkinje cells. Intermediate interneurons have morphological characteristics, which change without abrupt points of discontinuity along the molecular layer. 


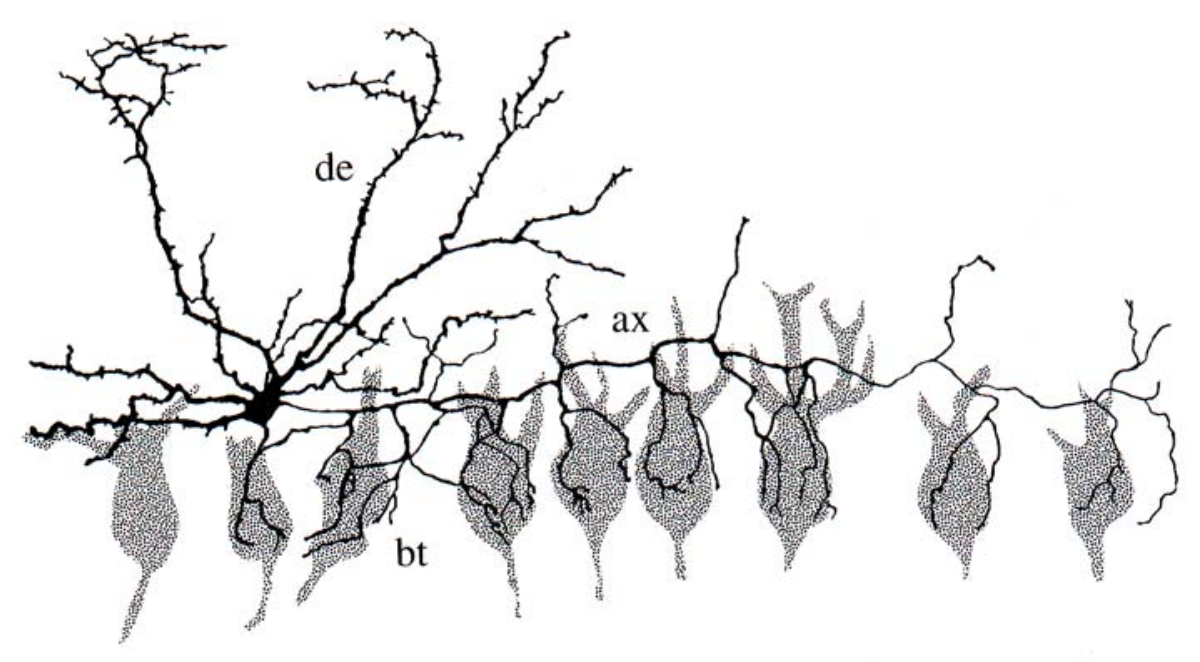

Figure 1.6: A basket cell from an albino rat in an illustration from Ramon y Cajal, 1911. The long, sagittal axon (ax) can be seen extending its collaterals around several consecutive Purkinje cells. Its dendrites (de) extend vertically throughout the molecular layer and are covered with spines; bt = basket cell terminal areas.

Stellate/basket cell dendrites receive glutamatergic synapses from parallel fibers on spines; the strong excitation produced in interneurons by these glutamatergic inputs gives the well described disynaptic feed forward inhibition recorded in Purkinje cells in vivo after parallel fiber stimulation (Eccles et al., 1966). The enormous advantage that this morphology offers in order to study the properties of basket/stellate cell to Purkinje cell synapses is provided by the "monodirectionality" of GABAergic axons; after originating from the cell soma or, in some cases, from primary dendritic arborizations, axons run through the molecular layer, making synapses on their way and always running linearly in the same sagittal plane (see Fig. 1.6). As it can be deduced, coplanarity of pre and postsynaptic structures and monodirectionality of the axonal course are great advantages when electrophysiological recordings of synaptically connected cell pairs are envisaged. In my experiments, the probability that two chosen cells are connected amounts to about $50 \%$ of the trials, which is remarkably high for this kind of experiments in a CNS area. vv 

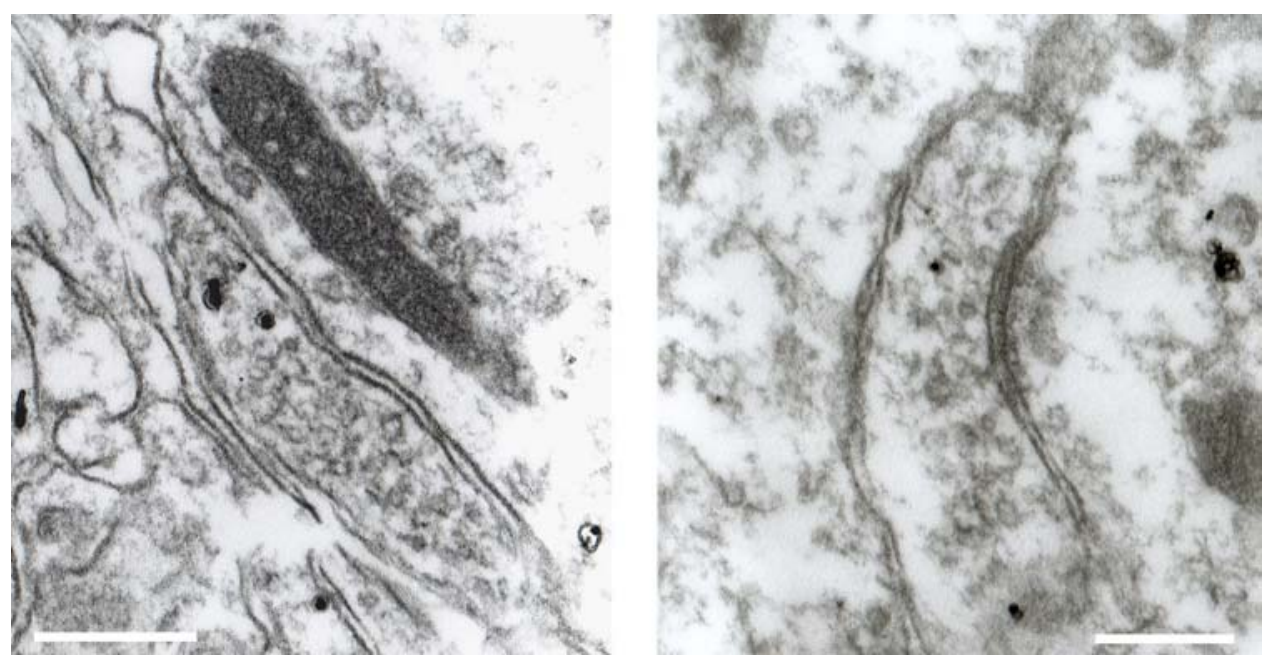

Figure 1.7: EM images taken from a 17 days old mouse. 2 symmetric synapses impinging on the soma (left) and on the dendrite (right) of a Purkinje cell can be observed. Putative basket/stellate cell to Purkinje cell synapses like these are characterized by the presence of flat oval vesicles and by pre- and postsynaptic thickenings of roughly similar widths; they are typically thought of being inhibitory. On the left, the section was likely cut slightly out of the plane comprising the active zone. Black dots are silver-enhanced gold particles showing the localization of the calcium binding protein parvalbumin; Bars $=1 \mu \mathrm{m}$.

\subsection{The basket/stellate cell to Purkinje cell synapse: a closer look}

Figure 1.7 is an EM image, which shows putative basket/stellate cell terminals onto a Purkinje cell in a young mouse. The microscopic appearance of GABAergic synapses is completely different from that of glutamatergic ones (as seen in Fig.1.5); inhibitory synapses are symmetrical (they do not show electron dense material on the postsynaptic side), they are characterized by flattened, oval vesicles and are normally not located on spines, but rather on smooth somatic or dendritic membrane surfaces.

Basket cell collaterals can form powerful synaptic connections with Purkinje cell somas, consisting of en passant boutons with several, clearly distinguishable active zones, while stellate cells preferably synapse with smaller terminals on dendritic structures (Altman and Bayer, 1997).

Cerebellar stellate/basket cells are spontaneously active: in in-vivo conditions, they fire at rates varying between 7 and $30 \mathrm{~Hz}$ (see for example Eccles et al., 1966); this spontaneous activity is mantained even in the slice preparation, quantitatively at physiological temperatures (Hausser and Clark, 1997) and at reduced rates (3 to $10 \mathrm{~Hz}$ ) at room temperature. The in-vitro basal, 
spontaneous firing of these cells is not dependent on synaptic inputs in terms of absolute rates, but rather in terms of regularity: pharmacological block of glutamatergic inputs induces only a negligible change in the firing rate (Clark and Hausser, 1997), mainly due to the fact that, in in-vitro conditions, excitatory inputs are mostly inactive. On the contrary, the activation of parallel fibers leads to a strong increase in interneuron firing rates, both in in-vivo (Eccles et al., 1966) and in in-vitro conditions (Carter and Regehr, 2000).

Similarly, the pharmacological block of GABAergic synaptic currents leads to a slight increase in fire frequency and to a more regular pattern of discharge. However, the important remark here is that stellate/basket cells are spontaneously firing neurons, that their pattern of activity is similar both in in-vitro and in in-vivo conditions and that synaptic activity is, thus, used to modulate the temporal profile of GABAergic input onto Purkinje cells. Consistently with the previous data, both in-vivo and in slices, electrophysiological recordings show a high frequency of spontaneous events with pharmacological and ionic properties typical of GABAergic currents and which can be related to the spontaneous interneuron firing activity (Eccles et al., 1966; Vincent and Marty, 1993 in Purkinje cells; Llano and Gerschenfeld, 1993 for GABAergic synapses onto stellate/basket cells).

What are the principal physiological properties of the synapses between stellate/basket and Purkinje cell?

A careful answer to this question must be given while keeping in mind the extremely important changes, which take place during the development of the rat cerebellum.

\subsubsection{The basket/stellate cell to Purkinje cell synapse in a developmental perspective}

During the first 4 postnatal weeks, the cerebellar cortex experiences great changes at all levels: Purkinje cells develop their impressive dendritic tree up to the 30th day after birth, granule cells migrate from the external cortical area along Bergmann glia neurites to reach their final destination in the granular cell layer and to finally proceed in the extension of their parallel fibers; stellate/basket cell are generated, migrate and develop in the first three weeks. The molecular layer increases remarkably in size, consistently with the developing extension of Purkinje cell dendrites, while being occupied by GABAergic interneurons: both types originating from precursors cells in the white matter, basket cells develop first (mostly between the 2nd and 11th day of life), followed by the more external stellate cells (between the 5th and 17th day). The final position in the molecular layer is reached at around the 21 st day after birth (Altman and 
Bayer, 1997).

Consistently with this developmental pattern, the number of basket/stellate cells per Purkinje cell increases with time, reaching a total value of at least 10 in adulthood (Korbo et al., 1993); although no quantitative data are available, also the number of synaptically connected GABAergic interneurons per Purkinje cell should have a similar positive pattern with time.

Under a strictly physiological point of view, the strength of the interneuron to Purkinje cell synaptic connections also changes with development: from high release probability synapses at age 11-15, characterized by paired-pulse depression (even if I will show that my data do not support the view of depressing synapses at this stage), by a low failure frequency and by a low coefficient of variation $(\mathrm{CV})$, they gradually change in time to become facilitating connections at high failure frequency (around 30\%) and with high CV (Pouzat and Hestrin, 1997). These presynaptically located modifications in synaptic function are associated with morphological changes like the shortening and fragmentation of basket cell active zones onto Purkinje cells (Larramendi, 1969), a decrease in the density of axonal varicosities (which are likely to be areas of accumulation of voltage-dependent calcium channels, and thus putative synaptic areas: Forti et al., 2000) and a general reorganization of all the contact areas with Purkinje cells: for example, climbing fibers to PC contacts translocate in the first three weeks from mainly somatic regions to more external, dendritic ones and are gradually replaced by basket cell axon collaterals (Altman and Bayer, 1997); in the following, these also colonize the PC axon hillock where they form the pinceaux specializations. Importantly, this happens at later stages than the time of my experimental activity.

Moreover, at an EM analysis GABAergic synapses start appearing frequently on basket/stellate cell soma only at the beginning of the second postnatal week (Altman and Bayer, 1997): thus, during development, the whole axonal plexus of these interneurons is likely to be subjected to a heavy remodelling process.

All the quoted physiological parameters support the hypothesis of a strong decrease in the release probability with development, taking place independently from the impressive changes in PC postsynaptic morphology (for example, filtering problems from unclamped compartments, as the adult PC dendritic trees actually are, do not contribute to the developmental changes observed with electrophysiological recordings: Pouzat and Hestrin, 1997); this does not anyway exclude that eventual, concomitant changes in postsynaptic responsiveness to GABA might take place, since no information is available about the regulation of GABA receptor subunit compo- 
sition with growth at these synapses.

Although this description induces many questions and doubts about the generalization of physiological findings obtained at early developmental stages to mature conditions, there are advantages in using young animals (11 to 15 days old in my case) which cannot be overlooked. Besides general euristic considerations, like the remark that slices from juvenile rats are usually healthier and conserve a higher number of surviving cells on their surface, the limited extension of the PC dendrites allow a much better control of the cell voltage in whole-cell conditions and, thus, a better quality of the electrophysiological recordings; the higher release probability at basket/stellate cell to PC synapses makes the signal to noise ratio a rewarding element for studying phenomena of synaptic plasticity, where big, reversible changes in synaptic responsiveness are to be quantified.

Moreover, as far as synaptic plasticity phenomena like DSI are concerned, we know that DSI is present also in the adult cerebellum (R. Rivosecchi, personal communication) and that it is present with the same pharmacological characteristics in the adult hippocampus.

Thus, in our case, developmental considerations appear to be all but of scarce influence in generalizing our results to adult animals.

\subsection{A few words on electrical synapses}

A further characteristics of cerebellar basket and stellate cells, which accumunates them to several GABAergic neurons in the CNS, is the presence of direct electrical synapses or gap junctions; electrical synapses are specialized sites where gap-junction channels bridge the plasma membranes of two adjacent neurons.

Very briefly, gap junctions are formed by proteins called connexins; these are membrane spanning proteins, six of which combine to give hemichannels known as connexons. Two connexons on two apposing plasma membranes can align to form intercellular channels, which then cluster to give the organized structures that we call gap junctions (for a review on connexins: White and Paul, 1999; see Fig.1.8).

By providing a low-resistance pathway for ions and and small molecules, electrical connections permit the direct transmission of electrical signals (what is also known as electrical coupling) between nerve cells. There are very important functional implications from the presence of 

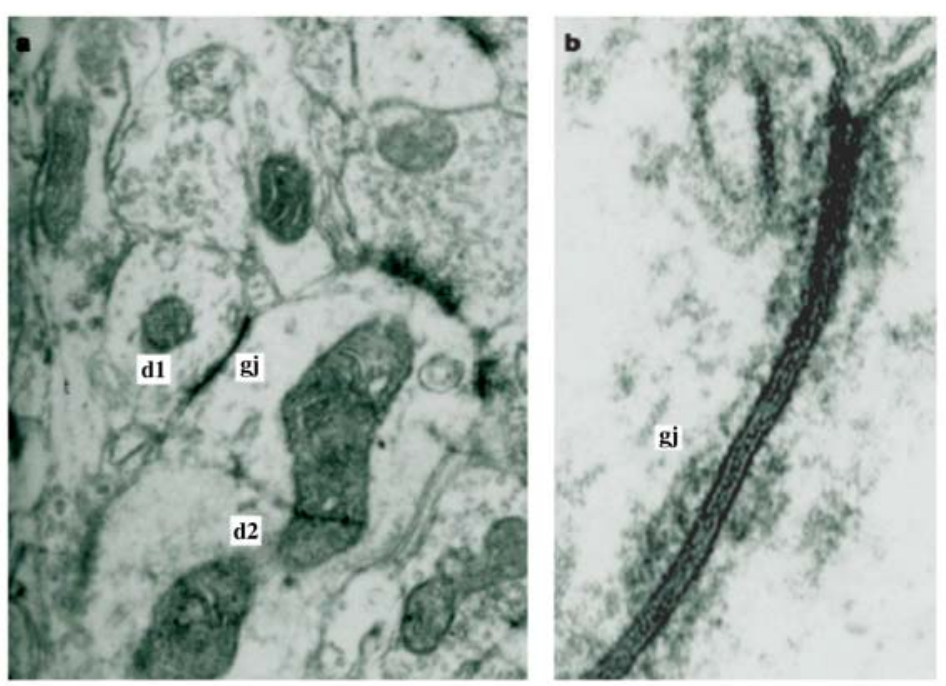

Figure 1.8: Gap junctions between dendrites of inhibitory neocortical interneurons. A gap junction (gj) is shown at different magnification (a: $\times 25,000$; b: $\times 180,000)$ between two dendrites of basket cells $(\mathrm{d} 1$ and d2 in a) of the adult primate motor cortex. B shows the typical multilayered structure of these connections and the accumulation of dense material nearby in the dendritic cytoplasm. (From Galarreta and Hestrin, 2001). 
electrical synapses between neurons: gap junctions were found to help mediate the synchronization of firing of GABAergic interneurons (Galarreta and Hestrin, 1999, Gibson et al., 1999; Mann-Metzer and Yarom, 1999). Thus, they are likely to play a fundamental role in the network oscillations, which interneurons have been found to generate both in theoretical (Traub et al., 2001) and experimental models (Michelson and Wong, 1994 and Whittington et al., 1995; Beierlein et al., 2000; see for a review on all these subjects: Galarreta and Hestrin, 2001). As far as the cerebellum is concerned, these specialized junctions have been described both morphologically (in adult cats and rats: Sotelo and Llinas, 1972) and, very recently, also physiologically in the adult guinea pig cerebellum (Mann-Metzer and Yarom, 1999). These data notwithstanding, the actual importance and, especially, the rate of occurrence of gap junctions is a long-standing open point in cerebellar interneurons from rat brain; I will show in the result sections that electrical synapses are actually a common finding in stellate/basket cells and I will try to discuss their role in connection with DSI and with cannabinoid action. 


\section{Chapter 2}

\section{Synapses and Retrograde Messengers}

The classical concept of interneuronal communication holds that information flows from the axon terminals of the presynaptic neuron to the dendrites of its postsynaptic partner.

This idea comes from the early, pioneering work of Santiago Ramon y Cayal: he proposed the "law of dynamic polarization", which, in the 1995 English re-edition of his masterful 1911 book (Ramon y Cajal, 1995) is stated as follows:

"Does this mean that incoming and outgoing impulses...pass indiscriminately from cell body to cell body, from one dendrite to another, from one axon to another, or from one of these three elements to any other? Or, conversely, is there some well established and immutable rule that determines what parts of one neuron may contact another cell? Everything we know about the function of the nervous system points to the latter supposition as true, and indicates that there is infact such a law. Our own observations have led us to define this law as follows:

a functional synapse or useful and effective contact between two neurons can only be formed between the collateral or terminal axonal ramifications of one neuron and the dendrites or cell body of another neuron."

We now know that Ramon y Cajal's use of the word "only" in the previous quotation is not entirely accurate and that, most likely, he was aware of the possibility of pushing his conjecture too far: indeed, already at the time he had recognized that most invertebrate neurons are unipolar and that, for example, vertebrate granule cells in the olfactory bulb and retinal amacrine cells lack axons.

In a second set of exceptions, recent results established that neurons are able to release neuro- 
transmitters from structures closely resembling "classical" axonal synaptic terminals but which are, according to today's identification criteria, dendritic. For example, in the external plexiform layer of the olfactory bulb, granule cell dendritic spines and mitral cell dendrites are reciprocally connected (Shepherd, 1998). A second example comes from the thalamus, where GABAergic so-called F-terminals make vesicle-containing asymmetrical contacts with dendrites of principal relay cells, thus mediating feed-forward inhibition of retinogeniculate excitatory inputs and of parabrachial cholinergic inputs (Sherman and Guillery, 2001).

In addition, many central neurons appear to be capable of releasing neurotransmitter-like substances from less organized areas of their somatodendritic compartment; these can have a strong modulatory influence on the activity of afferent neurons. These substances are able to modulate neurotransmitter release from afferent presynaptic terminals and include dopamine (Cheramy et al., 1981; Jaffe et al., 1998), dynorphin (Drake et al., 1994), glutamate and GABA (Zilberter, 2000; Zilberter et al., 1999), oxytocin and vasopressin (Kombian et al., 1997), BDNF (Korte et al., 1996) and, as I will describe thoroughly later, endogenous cannabinoids.

Modulating synaptic input in a direction opposite to that proposed by Cajal, these substances can therefore be defined as "retrograde messengers".

\subsection{Depolarization-induced Suppression of Inhibition (DSI)}

Retrograde signalling has been well established for Depolarization-induced Suppression of Inhibition (DSI), a form of short-term plasticity of GABAergic synapses onto cerebellar Purkinje cells (Llano et al., 1991; Vincent et al., 1992), hippocampal CA1 pyramidal cells (Pitler and Alger, 1992; Pitler and Alger, 1994) and cultured rat hippocampal neurons (Ohno-Shosaku et al., 1998).

DSI is triggered by postsynaptic mechanisms: depolarizations of a Purkinje cell in the cerebellum (Llano et al., 1991) or of a CA1 pyramidal cell in hippocampal slices (with depolarizing pulses in voltage-clamp: Pitler and Alger, 1994 or through retrogradely propagating spikes: Pitler and Alger, 1992 and Morishita and Alger, 2001) give rise to an increase in intracellular calcium concentration, which triggers a transient inhibition of GABAergic currents, fully reversible in a range of a few tens of seconds at room temperature.

DSI is not a phenomenon limited to GABAergic synapses: a very similar form of inhibition, in terms of time course, postsynaptic calcium dependence, presynaptic expression and phar- 
macology, has been recently found for glutamatergic synapses made by parallel and climbing fibers onto Purkinje cells (Kreitzer and Regehr, 2001a) and by glutamatergic Schaeffer collaterals onto CA1 pyramidal cells (Ohno-Shosaku, 2002b). In analogy to DSI, this phenomenon has been called Depolarization-induced Suppression of Excitation (DSE).

The depolarization-induced suppression of incoming synaptic input is, thus, likely to be quite a general mechanism of activity dependent regulation of the information flow in the CNS.

\subsubsection{Calcium dependence of DSI}

The increase in intracellular calcium is both a necessary and a sufficient condition for DSI: indeed, postsynaptic dialysis with intracellular solutions containing the calcium chelators BAPTA and EGTA block the induction of DSI (Glitsch et al., 2000; Pitler and Alger, 1992) while, on the other side, postsynaptic calcium uncaging is sufficient to trigger the phenomenon in hippocampal neurons (Wang and Zucker, 2001; Wilson and Nicoll, 2001).

Where does the calcium necessary for DSI come from? Is calcium-induced calcium release important for DSI, since activation of intracellular stores is known to occur in Purkinje cells following depolarizating pulses (Llano et al., 1994)? Unfortunately, no pharmacological tests have been done directly addressing this latter question. Nonetheless, hippocampal DSI shows a dependence from the voltage reached during DSI-inducing protocols that closely superimposes with the activation curve for voltage-dependent calcium conductances (Lenz et al., 1998). The superposition of the dependence of DSI and calcium conductances upon voltage suggests a linear relationship between DSI and calcium currents, thus apparently excluding regenerative processes in the liberation of calcium and, implicitly, intracellular stores.

As for Purkinje cells, DSI strongly depends on the length and the number of the depolarizing pulses and on the extracellular calcium concentration (Glitsch et al., 2000); these elements suggest the importance of somato-dendritic calcium conductances (most likely of the P-type; Mintz et al., 1992) in elevating calcium and induce DSI. Nevertheless, calcium signals can get supralinear in response to Purkinje cell depolarization, in a way which is blocked by pharmacological agents interfering with the functioning of intracellular calcium stores (Llano et al., 1994); this suggests that part of the calcium necessary to produce the retrograde messenger for DSI may come from these stores, although a direct proof is still to obtain.

A further, interesting point about the calcium dependence of DSI concerns the differences between cerebellum and hippocampus; indeed, in cerebellar Purkinje cells, a much higher calcium 
chelating power of the intracellular solution is required to block DSI than in the hippocampus: in Purkinje cells, $40 \mathrm{mM}$ of the fast buffer BAPTA is required to decrease DSI from a control $65-70 \%$ to about $15-20 \%$, while the same concentration of the slower buffer EGTA reduces DSI significantly less; on the contrary, 10mM BAPTA is enough to fully inhibit DSI in hippocampal CA1 cells (Glitsch et al., 2000; Pitler et al., 1992).

These data can have more than one explanation: cerebellar DSI induction may require a lower increase in calcium (different metabolic pathways for the production of the retrograde messenger(s) might be involved), and/or calcium may act more locally in Purkinje cells than in hippocampal CA1 cells (for example, the distance between calcium sources and enzymatic machinery could be smaller in the former cell type); or, on the other side, simply a difference in the density of membrane voltage-dependent calcium conductances or in the endogenous calcium buffer capacity of the two cell types could not be excluded (but notice, in this regard, that the buffer capacity is around ten times higher in Purkinje than in CA1 cells: Fierro et Llano, 1996 and Helmchen et al., 1996).

The hypothesis of a distinct sensitivity to calcium is supported by measurements of DSI halfsaturation as a function of intracellular calcium: in the range of $200 \mathrm{nM}$ for dendritic signals and 40nM for somatic ones in Purkinje cells (Glitsch et al., 2000), as calculated by grading the length and amplitude of the DSI-inducing protocol, and in the micromolar range for pyramidal cells (Wang and Zucker, 2001), as calculated, in this latter case, in calcium uncaging experiments.

Another important point to underline is that, in Purkinje cells, a second form of GABAergic synaptic plasticity comes to be associated with higher increases in the intracellular calcium concentration than the ones required for DSI; this is called Rebound Potentiation (RP), it is induced by the same protocols used to saturate DSI and also by climbing fiber stimulation, and consists in a long-lasting potentiation of transmission (up to 75 minutes). RP is fundamentally distinct from DSI in its expression mechanisms, being a fully postsynaptic event, and it is blocked by dialysis with only 10mM BAPTA (Llano et al., 1991; Kano et al., 1992).

It is tempting to speculate that a precise hierarchy in the sign of GABAergic synaptic plasticity exists; moderate elevations in calcium concentration might selectively activate only the presynaptic, short-lasting decrease in synaptic inhibition given by DSI, while higher values would trigger DSI followed by the postsynaptic, longer lasting RP. In this way, Purkinje cell integration of incoming inputs could be tuned according to the previous postsynaptic history, 
considering that the activity of stellate/basket cells is fundamentally important to modulate the Purkinje cell membrane time constant, its input resistance (Hausser and Clark, 1997) and the dendritic calcium transients elicited by excitatory synapses (Callaway et al., 1995). DSI, for example, could open a short time window in which excitatory inputs impinging on electrotonically distant dendritic branches might be more effectively associated and integrated by the Purkinje cell; on the contrary, RP would work to further isolate synaptic inputs; in addition, it could reduce the potentially plastic effects on transmission given by excitatory input-induced calcium transients (as for LTD of parallel fiber synapses; Wang et al., 2000 and, for review: Ito, 2001). A lot of evidence supporting the existence of physiological paradigms able to modulate GABAergic transmission is available: for example, both climbing and parallel fiber activation leads to calcium augmentations in Purkinje cells (Konnerth et al., 1992; Midtgaard et al., 1993), with supralinear signals at single spine level in case of temporal coincidence of the two inputs (Wang et al., 2000); furthermore, high frequency bursts of parallel fibers can activate mGluRs (Batchelor and Garthwaite, 1993), release calcium from intracellular stores and lead to endocannabinoid production (Maejima et al., 2001), while, as mentioned, climbing fibers can induce RP. Thus, in principle, many possible physiological stimuli could be capable of selecting which form of GABAergic plasticity to switch on.

The pattern we have now is similar to a sophisticated mechanical gear, where many single constitutive parts have been dismantled and characterized: we still miss an instruction booklet allowing us to have a general view of the interaction of the single parts, that is, we still miss precise information about what is moving what and in which physiological conditions this can happen.

\subsubsection{DSI is expressed presynaptically?}

Although induced postsynaptically, DSI is a presynaptic mechanism; in the cerebellum, evidence for this comes from the very first paper characterizing DSI: Llano et al., 1991, showed that the calcium entry following Purkinje cell depolarizations leads to the decrease of the frequency of TTX-independent miniature synaptic currents (mIPSCs). The amplitude of these events (which are external calcium-independent, Llano et al., 2000) is not changed after DSI while, on the contrary, the postsynaptic sensitivity to exogenously applied GABA is augmented, even if with a time course and a duration different from DSI (it is the already mentioned phe- 
nomenon of Rebound Potentiation: Llano et al., 1991).

A more recent paper (Yoshida et al., 2002) shows that DSI is associated with an increase in the Paired Pulse ratio of extracellularly evoked IPSC; this is another element for a presynaptic expression, since changes in the Paired Pulse ratio are inversely associated with a change in the release probability (Manabe et al., 1993; Zucker, 2002).

Surprisingly, in CA1 cells neither mIPSC frequency nor amplitude are modified during DSI (Pitler and Alger, 1994), although this is a delicate point: hippocampal DSI concerns only a subset of stratum radiatum/pyramidale interneurons, which express CB1Rs and whose synaptic activity onto CA1 cells is mediated exclusively by $\mathrm{N}$-type calcium conductances (Wilson et al., 2001). It is not known what the percentage contribution of these interneurons to the overall recorded mIPSC population is; this means that DSI could affect only this subpopulation of events and that the effect could be statistically lost in the background of unaffected minis.

Consistently with this view, in their first studies characterizing hippocampal DSI, Alger's group used carbachol to activate presynaptic acetylcholine muscarinic receptors and increase the frequency of miniature GABAergic events; this experimental procedure led to the increase of the amount of DSI of spontaneous events obtained by depolarizing CA1 cells and, importantly, to a statistically significant (12\%; Pitler and Alger, 1994) decrease in the mIPSC frequency during DSI. It is possible that muscarinic receptor activation directly excites only or mainly the same subpopulation of interneurons which is affected by DSI, making the inhibition of minis statistically "visible" to analysis.

Nevertheless, when potassium is added to the external solution in TTX mIPSCs become calcium dependent and their frequency, but not amplitude, is inhibited during DSI (Wilson and Nicoll, 2001, although Alger et al., 1996 show different results).

Moreover, substituting strontium for extracellular calcium leads to the appearance of asynchronous IPSCs following evoked IPSCs, which are indistinguishable from TTX-insensitive quantal mIPSCs and which are reduced in number but not in size during DSI (Morishita and Alger, 1997).

Finally, postsynaptic responses to exogenously applied GABA are not modified by DSI (Pitler and Alger, 1992), the Paired Pulse Ratio increases (Wilson and Nicoll, 2001; but notice that a previous study reached the opposite conclusion: Alger et al., 1996) and the number of failures in eIPSCs given by weak extracellular stimulation increases during DSI (Alger et al., 1996). All these experiments strongly suggest a presynaptic locus of expression for DSI and are not con- 
sistent with a postsynaptic change in sensitivity to GABA and/or number of synaptic receptors.

\subsubsection{Which retrograde messenger(s) for DSI?}

The question arises of the nature of the information which is exchanged, in a direction opposite to synaptic transmission, between the two sides of GABAergic synapses during DSI: indeed, DSI induction is postsynaptic (the increase in intracellular calcium concentration), while its expression is presynaptic (a downregulation of release probability and/or a modulation of action potential shape or propagation effectiveness). This implies the calcium-dependent production of a messenger, which, retrogradely, can convert the postsynaptic depolarization into a presynaptic decrease in synaptic output.

As explained earlier, many substances can be released from somatodendritic structures in order to influence the output of surrounding, afferent fibers. Particularly, in the case of phenomena of short and long-term synaptic plasticity a number of molecules have been proposed as possible mediators. Hippocampal Long-Term Potentiation (LTP) is the most thoroughly studied example of a long term plasticity phenomenon (Bliss and Collingridge, 1993); at present, the hypothesis of a postsynaptic locus of expression for LTP has gained most of the consensus on the scientific community (Malinow and Malenka, 2002), nevertheless, in the first half of the nineties several pieces of evidence had been collected showing a role for freely diffusible molecules like Nitric Oxide (NO), Carbon Monoxide, Arachidonic Acid and Platelet Activating Factor (Williams et al., 1989; Kato et al., 1994; for a comprehensive review also dealing with retrograde communication during synapse development and maturation: Fitzsimonds and Poo, 1998), as well as, more recently, for the growth factor BDNF (Korte et al., 1995, 1996).

As for DSI, Glitsch et al., 1996 excluded several possible diffusible messengers like NO, adenosine and GABA, and found that glutamate was a plausible candidate. The application of the specific mGluR2 agonist DCGIV both mimicked and occluded DSI; consistently with this finding, the broad spectrum glutamate receptor agonist/antagonist AP3 significantly reduced the DSI of mIPSCs.

The interpretation of these data was further supported by similar findings in the hippocampus: group 1 mGluR agonists mimicked and occluded DSI in CA1 pyramidal cells and the mGluR antagonist MCPG significantly reduced DSI (Morishita et al., 1998); at the same time, these results can be obtained also by applying other endogenous excitatory amino acids like L-cysteine sulfinic acid, which is known to activate glutamate receptors and to be released by 
central neurons (Morishita and Alger, 1999). Furthermore, the involvement of presynaptic pertussis toxin-sensitive G-protein was proposed (Morishita et al., 1997), thus reinforcing the idea of a retrograde messenger working through the activation of presynaptically located G-protein coupled receptors.

Two more points in favour of the glutamate hypothesis are then to be stressed: it has been shown that classical neurotransmitters (glutamate from cortical pyramidal cells and GABA from cortical interneurons) can be released from somatodendritic compartments to retrogradely activate metabotropic receptors (mGluRs and GABAb, respectively); the sensitivity to clostridial toxins of these phenomena suggests a vesicular origin for these transmitters and an exocytotic-like release process (Zilberter et al., 1999; Zilberter, 2000). Furthermore, like many neurons, Purkinje cells contain millimolar concentrations of glutamate and isoforms of SNARE proteins different from those involved in "classical" synaptic vesicle fusion events, but which can mediate vesicle fusion in other specialized cellular compartments like in epithelial cell apical membranes (e.g. SNAP23, VAMP7 and syntaxin 3 are: Coco et al., 1999 and Galli et al., 1998).

In spite of these data, the glutamate hypothesis has never been totally convincing, for several reasons. The block of DSI by specific antagonists is incomplete in the hippocampus and extremely small in the cerebellum (on mIPSCs: Glitsch and Jack, 2001; on sIPSCs: R.Rivosecchi, personal communication). Moreover, AP3 is an extremely aspecific agent, thus its effects could result from an interference with presynaptic effector pathways it could share with DSI.

These facts have left the search for the retrograde messenger open.

My data dealing with this subject will be thoroughly described in the results section and in the discussion, nonetheless I can here say in anticipation that in the last two years the glutamate hypothesis has turned out to be incorrect: hippocampal, cerebellar, cultured cell DSI and cerebellar DSE of climbing and parallel fiber input onto Purkinje cells and of glutamatergic inputs onto CA1 cells are all mediated by endogenous cannabinoids produced by the principal cells and acting on presynaptic CB1 receptors (Wilson and Nicoll, 2001; Wilson at al., 2001; Kreitzer and Regehr, 2001a, 2001b; Ohno-Shosaku et al., 2001; Diana et al., 2002; Yoshida et al., 2002); on the other hand the use of specific antagonists of glutamate metabotropic receptors have been found unable to block DSI or DSE (Wilson and Nicoll 2002, Kreitzer and Regehr, 2001a, 2001b; Diana et al., 2002; Yoshida et al., 2002).

In the discussion section, I will describe the possible reasons suggested by several recent studies (Maejima et al., 2001; Varma et al., 2001; Ohno-Shosaku et al., 2002), why glutamate pathways 
could actually have a modulatory action on the cellular machinery producing cannabinoids, thus affecting also DSI and DSE.

\subsubsection{Action Potential propagation failures as (one of many) possible mechanisms for DSI}

What are the possible mechanisms by which DSI inhibits transmission?

Once given that a certain molecule is the retrograde messenger, this question is equivalent to asking what this molecule targets downstream its presynaptic receptors; in the section of this thesis devoted to cannabinoids I describe thoroughly what the different possibilities are: voltage-dependent conductances modulation (calcium and potassium channels, for example) and a direct action on the release machinery.

I just want to highlight here a series of experiments concerning DSI, which open the exciting possibility that DSI also affects the presynaptic spike rate.

Indeed, both in the hippocampus (Alger et al., 1996) and in the cerebellum (Vincent and Marty, 1993), the hypothesis of an axonal block of action potential propagation was proposed very early.

In the hippocampus, Alger et al., 1993, found that DSI was not associated with an increase in the Paired Pulse Ratio of extracellularly evoked GABAergic currents in CA1 pyramidal cells. As mentioned earlier, events leading to a change in release probability induce a change in the PPR; these authors' findings led them to search for mechanisms affecting transmission at steps upstream the calcium entry into terminals and the subsequent activation of the release machinery: one possibility is the block of action potential conduction along presynaptic axons. Moreover, aspecific agents whose targets include conductances involved in action currents, like 4-AP and veratridine, reduced DSI (Alger et al., 1996).

In the cerebellum, the evidence in favour of a block of conduction and/or an inhibition of firing generation is strong. Vincent and Marty, 1993, have shown that DSI spreads along the Purkinje cell layer: undepolarized Purkinje cells display a decrease in impinging GABAergic currents following the depolarization of a nearby cell. This form of spreading presents the following important properties:

- unstimulated cells are affected at distances up to $75 \mu \mathrm{m}$, well over the predictable ranges for passive diffusion, even though this distance is calculated between the somata of recorded Purkinje cells. Actual distances between cannabinoid release sources (like Purkinje den- 
drites) and targeted CB1Rs could actually be lower;

- only time-locked events are affected. In view of the peculiar geometry of the molecular layer, basket/stellate cell axons synapse on several consecutive Purkinje cells; thus, an action potential leads to synaptic currents in nearby cells contacted by the same interneuron which are correlated in time. It has been shown that DSI spreading is correlated with the percentage of the events in common between two neighbouring Purkinje cells (Vincent and Marty, 1993).

- TTX abolishes the inhibition of GABAergic currents in undepolarized Purkinje cells; thus, a mechanism directly dependent on the integrity of action potential generation and/or propagation is involved in the spreading of DSI.

In view of this evidence and of the possible mechanisms of regulation of ionic conductances by cannabinoids, the hypothesis of a direct action of cerebellar DSI on action potentials is likely and its study is part of this thesis work.

\subsubsection{Unresolved Questions}

Following are the points, which were still open at the beginning of this thesis work and which guided my experimental work:

1. Can cerebellar DSI properties be thoroughly investigated at the level of the synapses made by a single interneuron onto a Purkinje cell? In this way, can its presynaptic expression be fully proved?

2. Is the DSI of mIPSCs quantitatively and qualitatively exactly the same as the DSI of TTXsensitive synaptic current? That is: are there actually several mechanisms which, acting in parallel, account for the depression of GABAergic transmission?

3. What are these mechanisms?

4. Is inhibition of spiking frequency involved in DSI and, if this is the case, to what extent is it responsible for DSI?

5. What is the retrograde messenger? Is the "glutamate hypothesis" correct? 


\section{Chapter 3}

\section{The Cannabinoid System}

Cannabinoids have a long history of consumption for recreational and medical reasons.

On one hand, Marijuana, the common name for the product extracted from the plant Cannabis sativa, has been used for over 4000 years as a recreational drug for its mind-altering effects while the problems connected with its abuse are still a hot socio-political subject of debate in these days. On the other side, there are established therapeutic actions of cannabinoid substances, from antiemetics, analgesic, and anticonvulsant to appetite stimulating effects which make them important targets of scientific and medical study.

Cannabinoids have been shown to exert cellular effects on many organs, including the immune system, the liver and reproductive system, although most of the behavioural and pharmacological actions studied so far involve the central nervous system.

A thorough review of the actions of cannabinoids falls outside the scope of this thesis. Here, I will merely present a description of the cannabinoid system in the CNS, with a special focus for the implications on synaptic physiology.

\subsection{Cannabinoids and their receptors}

\subsubsection{Type 1 (CB1R) and Type 2 (CB2R) cannabinoid receptors}

Although the effects of cannabis have been known for a long time, our knowledge of the chemistry and physiology of cannabinoids is quite recent, starting around ten years ago; two types of cannabinoid receptors have so far been identified: CB1 in 1990 (Matsuda et al., 1990), and CB2 in 1993 (Munro et al., 1993). 
Both belong to the big superfamily of G-protein coupled receptors; G-protein coupled receptors possess seven transmembrane domains and, when not binding an agonist, they are uncovalently linked to trimeric (composed of the $\mathrm{G} \alpha, \mathrm{G} \beta$ and $\mathrm{G} \gamma$ subunits) G-proteins, themselves bound to a GDP molecule. The binding of an agonist leads to the detachment of the G-protein from the receptor, to the replacement of GDP with GTP on the G $\alpha$ subunit and to several downstream effects on different cellular systems, exerted by the $\mathrm{G} \alpha$, the $\mathrm{G} \beta / \gamma$ subunits and the receptor itself. It appears clear from this model that, in principle, G-protein coupled receptor activation could trigger a plethora of independent effects and, as described later, this could be the case for the action of CB1Rs on GABAergic synaptic terminals in the cerebellum. In particular, CB1 and $\mathrm{CB} 2 \mathrm{Rs}$ are coupled to the pertussis toxin sensitive Gi/o-protein subtype and, downstream to this and classically for this G-protein type, the $\mathrm{G} \alpha$ subunit is negatively coupled to the enzyme adenylate cyclase (Matsuda et al., 1990; Slipetz et al., 1995) and positively coupled to mitogen-activated protein kinase (Bouaboula et al., 1995).

While the CB2R is restricted to the periphery, where it has been found in the marginal zone of the spleen (Munro et al., 1993), in tonsils and in cells of the immune system (B-cells, monocytes and T-cells; see Munro et al., 1993; Galiegue et al., 1995), the CB1 receptor is one of the most abundantly expressed G-protein coupled receptors in the brain, besides being also localized on adrenal glands, heart, prostata, uterus, ovary, testis and on presynaptic sympathetic nerve terminals. Stressing the potential importance of this receptor for brain functionality, it can be mentioned that in many brain areas CB1R mRNA density is greater than that of physiologically important receptors such as neuropeptide receptors and is comparable to that of catecholamine and even of glutamate receptors (Herkenham et al., 1990). These results indicate a high potential relevance of cannabinoid effect for the understanding of brain function.

In the rat brain, the localization of CB1Rs has been well characterized on a subcellular level (see for example Herkenham et al., 1990 and 1991; Matsuda et al., 1993; Pettit et al., 1998, from which Fig.3.1 has been taken; Tsou et al., 1998; Egertova and Elphick, 2000) with immunohistochemical techniques. The receptor is present virtually in every brain area: very strong is the expression in the molecular layer of the cerebellum and, in particular, in parallel fiber terminals onto Purkinje cells, from which they are absent, and in the pinceaux regions of GABAergic basket cells terminals onto Purkinje cells (Tsou et al., 1998 and Fig.3.2 extracted from Egertova and Elphick, 2000); in the latter case, the quoted studies were done on adult animals and gave no further indication on CB1R presence on interneuron axons, two elements which prompted us 


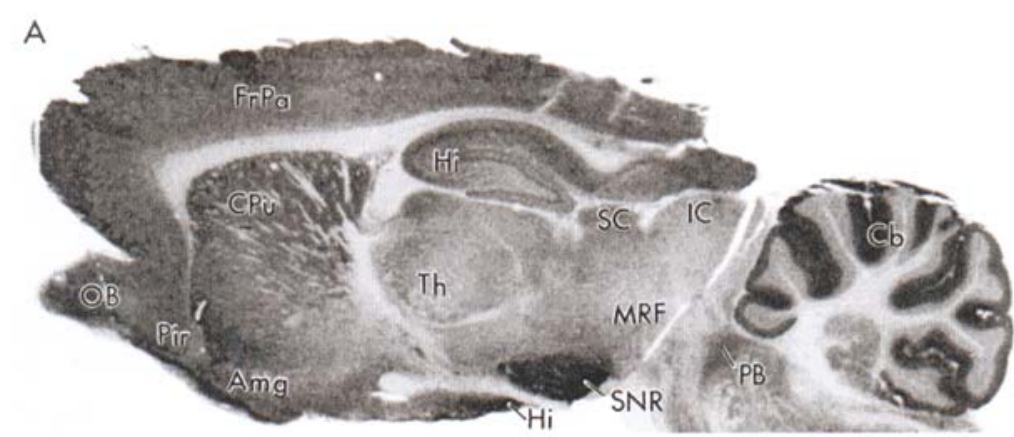

B

Figure 3.1: Immunohistochemical staining in sagittal sections of the rat brain using a CB1R specific antibody (A) and the same antibody preadsorbed with its corresponding peptide antigen (B); it can be seen the widespread distribution of this receptor in mammalian brain with particularly high levels of expression in the cerebellum (Cb), hippocampus (Hi), Substantia Nigra pars reticulata (SNR) and the basal ganglia (Caudate and Putamen: CPu). Scale bar $=1.42 \mathrm{~mm}$. From Pettit et al., 1998 .

to do immunocytochemical and electromicroscopy studies to collect further data in this regard. High levels can also be found, for example, in the basal ganglia (which, with the cerebellum, are likely to mediate the negative effects of cannabis on posture and locomotion) and in the hippocampus, where the receptor is present only on GABAergic interneurons that express even the peptide cholecystokinin (Katona et al., 1999 and Tsou et al., 1999) but not, for example, on glutamatergic neurons and terminals. Intensely stained neurons (even if in smaller amounts than in the hippocampus) and fibers (both dendrites and axons) can then be found almost everywhere, from other cortical structures like the cerebral cortex and the olfactory bulb, to the amygdaloid complex, the nucleus accumbens, the substantia nigra and lower areas like the spinal cord and the periaqueductal gray (importantly for the analgesic effects of cannabinoids). Consistently with the morphological and the behavioral data, physiological studies in the last years have been confirming the presence of functional CB1Rs in all these areas: CB1R activa- 


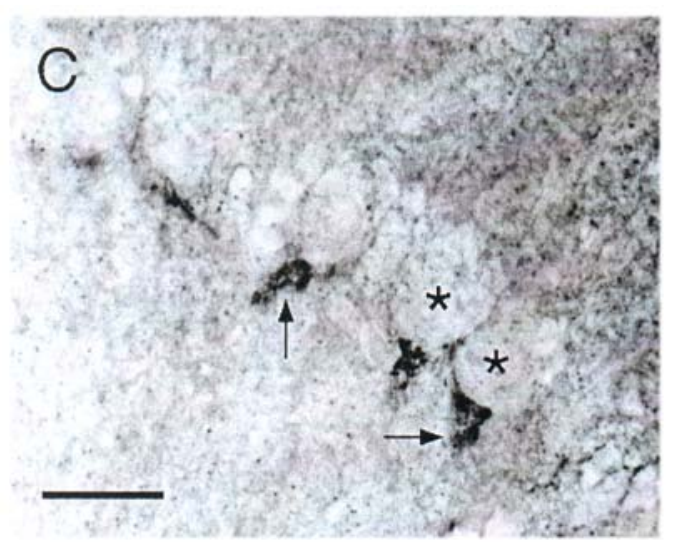

Figure 3.2: CB1 immunoreactivity in the cerebellum of the rat. While the molecular layer shows a uniform staining (not depicted here), in the Purkinje cell layer the staining is concentrated in cap-like structures around the basal part of Purkinje cell soma, the so called pinceaux which are formed by basket cell axons wrapping around the Purkinje axon hillock. Scale bar $=50 \mu \mathrm{m}$. From Tsou et al., 1998.

tion has been shown to inhibit GABA release in, for example, the rostral ventromedial medulla and periaqueductal gray (Vaughan et al., 1999, 2000), hippocampus (Katona et al., 1999; Hajos et al., 2000; Hoffmann and Lupica, 2000), cerebellum (Takahashi and Linden, 2000), nucleus accumbens (Manzoni and Bockaert, 2001), striatum (Szabo et al., 1998), substantia nigra pars reticulata (Chan and Yung, 1998); inhibitory effects on glutamatergic transmission have been shown in the mouse Nucleus Accumbens (Robbe et al., 2001), in hippocampal CA1 pyramidal cells (likely not through CB1Rs, but through a yet uncloned receptor, as explained later; Misner and Sullivan, 1999), in the cerebellum at the parallel fibers to Purkinje cell synapse (Levenes et al., 1998), prefrontal cortex (Auclair et al.,2000), substantia nigra pars reticulata (Szabo et al., 2000), periaqueductal gray (Vaughan et al., 2000), striatum (Gerdeman and Lovinger, 2001; Huang et al., 2001) and spinal cord substantia gelatinosa (Morisset and Urban, 2001).

It is important to underline the fact that CB1R activation ALWAYS exert an inhibitory action on synaptic transmission, be it GABAergic or glutamatergic, and that these effects are mediated by presynaptic mechanisms even in cases, like the striatum, where CB1Rs are present on both sides of corticostriatal glutamatergic synapses (Rodriguez et al., 2001; for a general review centered on the presynaptic aspects of cannabinoid action see Schlicker and Kathmann, 2001). 


\subsubsection{What about a CB3 receptor....}

Two papers have presented evidence in favour of the existence in the CNS of a so far uncloned, third cannabinoid receptor; actually, such a hypothesis could explain contradictory morphological and physiological data concerning, in particular, glutamatergic cells in the hippocampus. In the hippocampus, indeed, CB1 receptors are expressed only in cholecystokinin containing GABAergic interneurons (Tsou et al., 1999; Katona et al., 1999) and not in glutamatergic, principal cells; this is in flagrant contrast with several lines of physiological evidence, which in many cases show effects of exogenous cannabinoids both on CA1 pyramidal cells (like modulation of the potassium M current: Schweitzer, 2000; or a reduction in CA1 cell input resistance: Kirby et al., 2000) and on Schaffer collateral glutamatergic terminals onto CA1 cells (Ameri et al., 1999; Misner and Sullivan, 1999; Hajos et al., 2001 and Ohno-Shosaku et al., 2002b), where cannabinoids inhibit transmission with a pharmacological characterization typical of cannabinoid-like receptors (even if not coincident: THC, the active compound of Marijuana, does not activate these putative new pathways: the effects of cannabis assumption could, thus, be ascribed only to $\mathrm{CB} 1$ and $\mathrm{CB} 2 \mathrm{Rs}$ ).

Importantly, the effects on glutamatergic, but not GABAergic, transmission are still present in CB1R knock-out mice (Hajos et al., 2001), and, furthermore, membranes from many CNS areas of CB1R knock-out mice still bind radioactively-labeled GTP following application of the putative endogenous cannabinoid anandamide and of the high-affinity CBR agonist WIN55-212 (Breivogel et al., 2001); thus, the evidence suggests that a third, G-protein-coupled cannabinoid receptor might exist and might be important, for example, for the anticonvulsant actions of cannabinoids, seen its apparent specific connection with glutamatergic, excitatory pathways.

\subsection{3 -...and actions through other receptors?}

Finally, it must be remarked that the endogenous cannabinoid anandamide is also an agonist (even if with a smaller potency than in the case of CB1Rs) of a different family of channel proteins, the so called Vanilloid Receptors (VR; Caterina and Julius, 2001); these mediate many noxious responses in the spinal cord and the peripheral nervous system (for example, they signal the piquancy of hot chili pepper and they are sensitive to low $\mathrm{pH}$ environment and high temperature) and are also highly expressed in the CNS, for example in the cerebellum (for a review on this subject, see: Szallasi and Di Marzo, 2000). 
Two studies report about CNS effects of vanilloid receptor activation: the Paired Pulse ratio of population spikes recorded in the CA1 region of rat hippocampal slices is reduced by anandamide, and this action is blocked by a VR antagonist (Al-Hayani et al., 2001); moreover, anandamide and vanilloid agonists dramatically increase glutamatergic transmission in the locus coeruleus (Marinelli et al., 2002).

At present, vanilloid receptors are orphan receptors, that is: no endogenous ligand has been so far found for them. In various systems, there is a partial superposition between vanilloid and cannabinoid receptor localization and pharmacology; this is the case of anandamide and of the molecule AM 404, which is at the same time a VR agonist and a blocker of the cannabinoid transporter. Expanding the range of possible physiological implications of endogenous cannabinoids, vanilloid receptors could, thus, help explain central and peripheral phenomenona, whose properties are inconsistent with a "classical" cannabinoid system: this is the case for the CB1Rindependent inhibition of gap junctions by anandamide in astrocytes (Venance et al., 1995), and for the reported vasodilator effect of anandamide through sensory neurons (Zygmunt et al., 1999).

\subsection{Endogenous cannabinoids: identity, metabolism and uptake}

\subsubsection{Identity and metabolism}

The CB1 receptor was first identified in 1990 as an orphan receptor. Although its chemical structure was known since the beginning of the sixties, $\Delta 9$ (-)-tetrahydrocannabinol (THC), Marijuana's principal psychoactive ingredient, is not produced by mammalian organisms, so that even the existence of specific cannabinoid receptors was for many years considered uncertain: indeed, THC's lipophilicity and its cell-membrane disrupting actions could in principle account for marijuana's complex physiological effects.

The synthesis of a biologically active cannabinoid compound led to a first characterization of cannabinoid receptors (Devane et al., 1988), then to the cloning of CB1 (Matsuda et al., 1990) and to the identification of the first endogenous cannabinoid from the porcine brain: anandamide (AEA; from the sanskrit "bringer of inner bliss and tranquillity"), the amide of arachidonic acid (another putative messenger involved in the control of synaptic efficacy) and ethanolamine (Devane et al., 1992).

Other cannabinoid receptor ligands distinct from anandamide have been found since then (see 


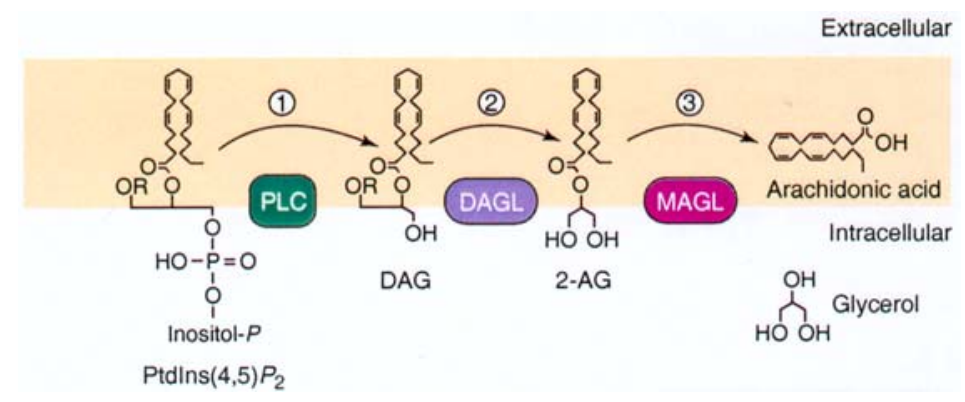

Figure 3.3: Proposed metabolic pathways for the formation and the inactivation of 2-arachidonylglycerol (2AG). Phospholipase C activation produces two second messengers: 1,2-diacylglycerol (DAG) and IP ${ }_{3}$; DAG then serves as a substrate for the enzyme DAG lipase to produce 2-AG. After being released extracellularly, 2-AG can bind to cannabinoid receptors and then transported intracellularly to be further hydrolised to arachidonic acid and glycerol by the enzyme monoglyceride lipase (MAGL; from Piomelli et al., 2000).

for a review Di Marzo et al., 1998): special attention is now devoted to 2-arachidonoylglycerol (2-AG), first isolated from dog intestine (Mechoulam et al., 1995).

Why has attention been focused particularly on AEA and 2-AG? The main reason is that these two molecules are produced in neuronal tissue (2-AG, after stimulation of Schaffer glutamatergic collaterals on pyramidal cells: Stella et al., 1997; AEA in the striatum following sopamine $\mathrm{D}_{2}$ receptors: Giuffrida et al., 1999) and cultured neurons (2-AG: Stella et al., 1997; AEA and/or its precursors: Di Marzo et al., 1994 and Cadas et al., 1996) in a calcium- and depolarizing agent- dependent way, thus suggesting possible physiological mechanisms of synthesis and release in the CNS.

The proposed metabolic pathways leading to their release are depicted in Fig.3.3 and Fig.3.4; importantly, experimental evidence in favour of these schemes has already been described (Cadas et al., 1996, 1997 and Stella et al., 1997).

Moreover, these ligands are not stored as classical neurotransmitters are; they are synthesized directly from lipid precursors present on cellular membranes and released in the extracellular space to activate CB1Rs on presynaptic terminals. This model assumes that endocannabinoids could be produced in neuronal somatodendritic compartments although this is still only a speculation: the uncertainty on this point mainly derives from the fact that the biosynthetic enzymes are still being investigated and no means actually exist to localize endocannabinoid products in subcellular areas.

The suggested biosynthetic pathways contain in both cases calcium-dependent steps: for anan- 
damide the enzyme $\mathrm{N}$-acyl transferase works in a calcium-dependent way (Cadas et al., 1996), while, for 2-AG (normally present at 170 times the amount of AEA in brain tissues: Stella et al., 1997), phosphoinositide-specific phospholipase C can be calcium-dependent (Rebecchi and Pentyala, 2000). These pieces of evidence notwithstanding, it is still an open question whether endocannabinoids can be produced also in a calcium-independent way and whether, where calcium is inequivocably involved, calcium concentration increases are by itself sufficient to induce synthesis. These considerations arise especially for the reported cases, in which the biosynthetic pathway is activated following G-protein coupled receptor activation, for example following mGluR1 receptor activation in Purkinje cells and CA1 hippocampal neurons (Maejima et al., 2001; Varma et al., 2001). The only case where calcium concentration increases seem "per se" to be the only requirement for cannabinoid production is during the induction of DSI (see the chapter specifically devoted to this phenomenon of synaptic plasticity); reviewing the remaining literature on the subject, this cannot be extended: the calcium ionophores and depolarizing agents used to characterize the calcium dependence of AEA and 2-AG productions are all agents that in neuronal tissues and dissociated cultures lead to aspecific, massive release of neurotransmitters from presynaptic fibers. Consistently, both in cultures (Cadas et al., 1996 and Stella and Piomelli, 2001) and in vivo (Giuffrida et al., 1999), AEA production depends on the integrity of action potential generation and on receptor activation: through dopaminergic $\mathrm{D}_{2}$ Rs in vivo (Giuffrida et al., 1999) and through combined NMDA and muscarinic receptors in cultures (and the following intracellular store activation: Stella and Piomelli, 2001); likewise, 2-AG is produced in hippocampal slices after stimulation of Schaeffer afferent fibers to CA1 pyramidal cells (Stella et al., 1997) and, in cultures, in an NMDA-dependent way (Stella and Piomelli, 2001).

Finally, AEA and 2-AG could also be produced in the same system independently from each other and in response to different receptor stimulations, something that could be fundamental in the fine tuning of the cannabinoid system in in-vivo conditions (Stella and Piomelli, 2001). In general, the scheme which has at present emerged is still largely incomplete in many important aspects, in spite of the ever increasing evidence indicating that AEA and 2-AG are endogenous neuroactive compounds. 


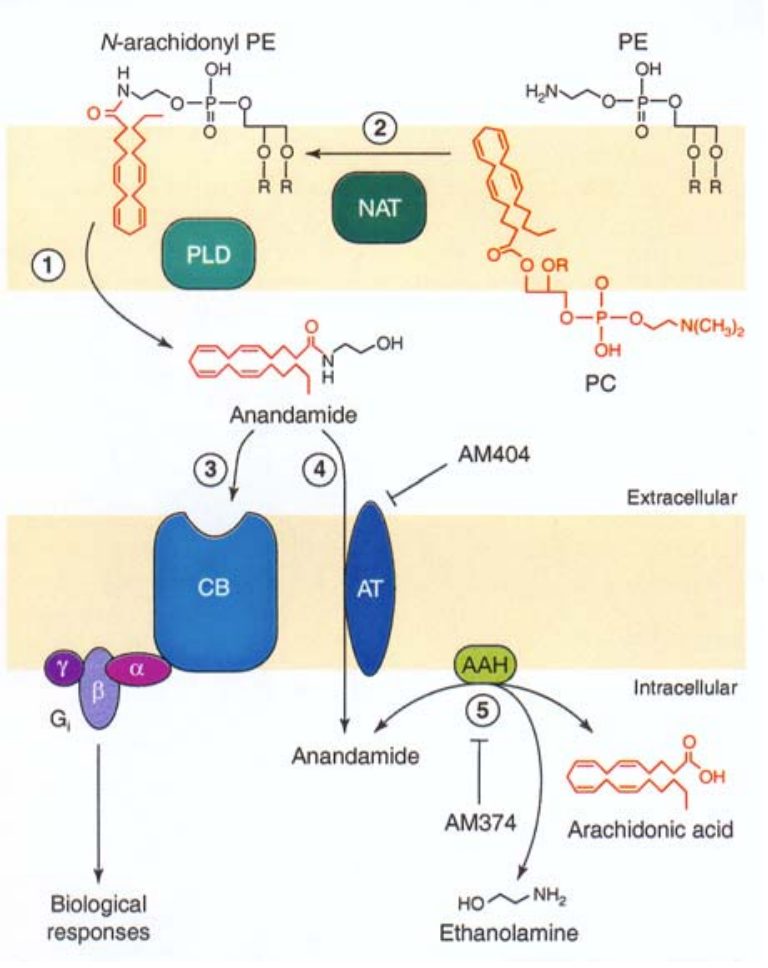

Figure 3.4: Formation and inactivation of anandamide. Phospholipase D (PLD) can catalyse the hydrolysis of $\mathrm{N}$-arachidonyl phophatidylethanolamine ( $\mathrm{N}$-arachidonyl PE or NAPE), which itself can derive from $\mathrm{N}$ acyl transferase (NAT)-mediated transfer of an arachidonate moiety from phosphatidylcholine (PC) to PE. Anandamide can then be removed from the extracellular milieu by a carrier-mediated transport and intracellularly degraded to ethanolamine and arachidonic acid by a fatty acid amide hydrolase (AAH). Notice that the membrane localizations of NAT and PLD are still speculative (From Piomelli et al., 2000). 


\subsubsection{Uptake}

Endogenous cannabinoids are partially lipophilic molecules which, after synthesis on membrane compartments, can be released and can diffuse extracellularly until actively recaptured and degraded. The degree of diffusion is likely to depend on the structure of the extracellular matrix and on the localization of the putative specific transporter responsible for the $\mathrm{Na}$ independent, active uptake of anandamide and 2-AG (Beltramo et al., 1997; Beltramo and Piomelli, 2000); the existence of such a transporter is suggested by the high affinity, temperature dependence, substrate specificity and substrate saturation of the process of endocannabinoid accumulation in glial cells and neurons.

Consistently, the uptake inhibitor AM-404 has been shown to prolong several pharmacological responses elicited by anandamide (e.g. vasodepressor responses in Calignano et al., 1997) or to reveal a basal production of endocannabinoids (CB1R-dependent inhibition of synaptic transmission in hippocampal slices: Wilson and Nicoll, 2001).

Evidence from physiological experiments suggests that endocannabinoid signalling might be an extremely local phenomenon, limited to less then $20 \mu \mathrm{m}$ from the source (Wilson and Nicoll, 2001) in the hippocampus, or even with undetectable diffusion, as in the case of mGluR- induced cannabinoid production in cerebellar slices (Maejima et al., 2001).

Once transported intracellularly, cannabinoids can be degraded by the enzyme fatty acid amide hydrolase (FAAH; Cravatt et al., 1996), which, unsurprisingly, shows a complementary expression to fibers possessing CB1Rs: for example, in the cerebellum, FAAH is highly expressed by Purkinje cells, from where CB1Rs are absent (Tsou et al., 1998b; Egertova et al., 1998); FAAH can thus be involved in the cannabinoid signaling system, as suggested by the biochemical suggested schemes of formation and inactivation for these substances. This is supported by the supersensitivity to anandamide and to the 15-fold higher levels of this compound in knock-out mice for FAAH (Cravatt et al., 2001).

Finally, the enzyme brain monoglyceride lipase (MGL) has recently been suggested to partecipate in the selective degradation of 2-AG, but not of AEA, in cortical neurons, thus widening the spectrum of possible degradative pathways for cannabinoids in the CNS (Dinh et al., 2002). 


\subsection{Cannabinoids and calcium channels}

A primary target of action in regulating synaptic transmission for endogenous cannabinoids are voltage-dependent calcium channels. Similarly to many receptors coupled to pertussis toxinsensitive $\mathrm{G}$ proteins, CB1Rs inhibit $\mathrm{N}$ and $\mathrm{P} / \mathrm{Q}$ type calcium conductances in several cell lines, from expression systems (Caulfield and Brown, 1992; Mackie and Hille, 1992; Mackie et al., 1993; Mackie et al., 1995) to primary neurons (Pan et al., 1996; Twitchell et al., 1997). Further evidence was obtained in pharmacological occlusion experiments between calcium conductance toxins and exogenous CB1R agonist application: in some cases, cannabinoid-mediated synaptic depression could be explained by a selective targeting of the calcium currents responsible for synaptic transmission (N-type channels in GABAergic interneurons to CA1 pyramidal cell synapses: Wilson et al., 2001; simultaneous Q- and N-type inhibition in cultured glutamatergic autapses: Sullivan, 1999); in others, the use of more aspecific blockers has suggested a role for these conductances in synaptic inhibition, though not providing specific information on the involved isoforms (see in the hippocampus: Hoffman and Lupica, 2000; in rat striatum: Huang et al., 2001).

Using imaging techniques, Kreitzer and Regehr, 2001a, have shown that endogenous cannabinoids lead to a decrease in calcium entry per action potential in cerebellar climbing fiber terminals; though suggesting a role for calcium conductances, these experiments cannot still exclude other mechanisms leading to the same effect, as reviewed later in this chapter.

As for basket/stellate cells to Purkinje cells synapses: transmission in these structures is mostly mediated by P/Q-type channels (as assessed pharmacologically on transmission by Stephens et al., 2001 and on axonal action potential-induced calcium transients by Forti et al., 2000) and CB1Rs are able to down-regulate these conductances; it is thus plausible that at least part of the transmission from these GABAergic synapses might be inhibited in this way.

It is then worth mentioning two more facts concerning $G$ protein-mediated inhibition of calcium conductances:

1. the modulation is exerted through the direct interaction between the $\mathrm{G} \beta / \gamma$ subunits and the channel protein (Herlitze et al., 1996; Ikeda, 1996) and the process is cAMP-independent (the inhibition of adenylate ciclase takes place through the $\mathrm{G} \alpha$ subunit; see later for the possible consequences on the functionality of our synapses of the CB1R-induced inhibi- 
tion of adenylate cyclase);

2. N-type channels are twice as sensitive to inhibition by G-protein as P/Q conductances ( Zhang et al., 1996; Currie and Fox, 1997). This could lead to a different percentage of GABAergic synaptic inhibition attributable to calcium channels in the hippocampus and in the cerebellum; consistently, while in the former case all of the CB1R effect on evoked transmission could be simply explained by N-type blockade (see Wilson et al., 2001), in the cerebellum there are several elements which suggest the involvement of several independent mechanisms in parallel.

\subsection{Cannabinoids and potassium channels}

\subsubsection{Influence of presynaptic $K^{+}$channels on synaptic strength}

A further possibility for cannabinoids to modulate synaptic transmisission is through the upregulation of potassium channels.

Several voltage-dependent potassium channels have been cloned and characterized: the remarkable diversity of subunits underlying these currents allows the formation of channels with very different biophysical and pharmacological properties (see for a very extensive review: Coetzee et al., 1999).

Every cellular type in the CNS expresses a unique pattern of potassium conductances: this sets in large part the differences between cell types in action potential waveform, in its modulation in periods of high frequency firing and in the range of admissible spike frequencies. Furthermore, several of these conductances can be modulated by second messenger-mediated pathways, making the action potential waveform a possible target for the action of neuromodulators.

The activation of potassium conductances can affect synaptic activity in several ways:

1. Limiting the duration of action potentials: the shape of the action potential has a fundamental importance in determining the synaptic strength; indeed, action potential-induced synaptic transmission is triggered by calcium ions entering the cell at the depolarized potentials reached during firing (Katz and Miledi, 1967; Dodge and Rahamimoff, 1967). By limiting the action potential duration, voltage-dependent potassium channels lead to the deactivation of calcium conductances, to the interruption of the calcium flow and, thus, of 
evoked transmission. Since synaptic transmission is dependent on the amount of calcium entry with a supralinear dependence on calcium (Dodge and Rahamimoff, 1967; Augustine and Charlton, 1986; Mintz et al., 1995; Bollmann et al., 2000; Schneggenburger and Neher, 2000), it follows that any manipulation leading to a small modification of the repolarization phase of an action potential can have dramatic effects on neurotransmitter release; this has already been shown in several different systems (see Augustine, 1990; Coates and Bulloch, 1985; Delaney et al., 1991; Klein and Kandel, 1982; Spencer et al., 1989; and more recently: Sabatini and Regehr, 1996; Vyshedskiy et al., 2000; Geiger and Jonas, 2000) and in some of these cases a direct role for potassium conductances has been found (Siegelbaum et al., 1982 and Hoechner et al, 1986 in Aplysia; Sabatini and Regehr, 1997; Vyshedskiy et al., 2000) or suggested (as in Jackson et al., 1991 and Geiger and Jonas, 2000).

2. Through a direct action on the release machinery: this is for example the suggested scenario for the inhibitory actions of opioids on GABAergic transmission in the Periacqueductal Gray (PAG; Vaughan et al., 1997), where $\mu$ - opioid receptors inhibit the frequency of calcium-independent mIPSCs by activating an $\alpha$ DTX - sensitive potassium conductance. Particularly interesting is that, in these terminals, $\mu$ receptors are coupled to the potassium conductance through a pathway which involves arachidonic acid and/or derivatives; although metabolically distinct, these molecules are chemically close to the endogenous cannabinoids $2-\mathrm{AG}$ and anandamide.

3. Blocking the generation and/or the conduction of action potentials in axons; this possibility is raised by previous theoretical (see for example Segev, 1990) and experimental work (Macagno et al., 1987; Luscher et al., 1994a/b and Debanne et al., 1997), although other studies in acute slices failed to reveal conduction failures using imaging techniques (for example in Forti et al., 2000; and Cox, et al., 2000; Koester and Sakmann, 2001; Kreitzer and Regehr, 2001a). This inhibitory effect on firing by cannabinoids might in principle take place both through shunting effects due to local decreases in membrane resistance and/or to local hyperpolarizations. 


\subsubsection{Reported effects of CB1R activation on potassium conductances}

\section{Rapidly inactivating I(A) currents:}

As far as the rapidly inactivating I(A) current is concerned, in cultured hippocampal neurons, cannabinoids produce a shift towards more positive voltages of the inactivation curve, thus leading to an increased activation of I(A) near resting values (Deadwyler et al., 1993); this phenomenon is dependent on pertussis toxin and on the cAMP pathway (Deadwyler et al., 1995), in particular on PKA activity (Hampson et al., 1995). Nonetheless, regarding these data, a point must be underlined: the apparent contradiction between the assignment of these effects to activation of CB1Rs and the fact that all the available morphological evidence goes against the presence of CB1Rs on hippocampal glutamatergic neurons; as already mentioned, an explanation for these contrasting reports can come from the likely existence of a so far uncloned CB receptor (Hajos et al., 2001).

\section{Inwardly rectifying GIRK channels:}

The four different GIRK channel isoforms (see Coetzee et al.,1999) giving rise to inwardly rectifying potassium conductances are important elements in the control of cellular excitability: ubiquitous, they work by hyperpolarizing the membrane potential in cardiac and secretory cells and in neurons. Inwardly rectifying channels can be activated by cannabinoids in expression systems (Mackie et al., 1995; Henry and Chavkin, 1995; Ho et al., 1999), in a pertussis toxin sensitive way and by direct binding of G-protein $\beta / \gamma$ subunits (Reuveny et al., 1994; and Krapivinsky et al., 1995 for the heteromeric GIRK1-GIRK4 heart channel).

In particular Ho et al.,1999 have described a positive interaction between the cannabinoid pathway and heteromeric inwardly rectifying conductances composed of GIRK1 and GIRK4 subunits; extremely interesting is then the high level of expression of the GIRK4 subunit in the cerebellar pinceaux, and, thus presumably along molecular layer axons (Iizuka et al., 1997). The activation of GIRK4-containing channels could lead to transient local shunting effects and effects both on action potential reliability of propagation and its waveform, thus contributing to the inhibition of synaptic transmission occurring during DSI.

\section{Studies in acute slices:}

All the previous studies have been done in cultured cells or neurons, an element that strongly limits their physiological significance; in more physiological conditions, two recent reports suggest that CB1R-mediated inhibition of transmission is given by the activation of voltage- 
dependent potassium conductances: Robbe et al. (2001) have shown that the inhibitory action of exogenous cannabinoids on glutamatergic transmission in mouse Nucleus Accumbens slices is blocked by application of the aspecific potassium channel blockers barium and 4-AP, and that this block is mantained when synaptic transmission is brought back to control levels by decreasing the extracellular calcium concentration (this is an important control, since application of these agents leads to a broadening of the action potential, an increase in calcium in-flow and a strong increase in synaptic transmission; this implies the possibility that the decreased effectiveness of cannabinoids might be due to the inability of the CB1R activated pathways to counteract these potentiating effects). Interestingly, all this happens independently from the cAMP-PKA cascade.

Similarly, Daniel and Crepel (2001) have shown in cerebellar slices that action potential-induced calcium transients in parallel fibers are inhibited by WIN55,212-2 application, and have proposed that this effect contributes to the synaptic inhibition at these synapses by CB1Rs (see Levenes et al., 1998 and Takahashi and Linden, 2000). Also in this case, bath application of 4-AP blocks the effect of WIN and control experiments similar to those of Robbe et al., 2001 seem to exclude a saturation problem.

In contrast with these studies, there are two other recent studies performed on hippocampal GABAergic neurons in slices: Hoffmann and Lupica (2000) showed that the CB1R-induced depression of GABAergic transmission onto CA1 pyramidal cells, which is fully blocked by bath applied barium and 4-AP, is recovered when decreasing extracellular calcium, thus excluding potassium channels from the cannabinoid effect.

The two articles above are the only elements so far to build a bridge between CB1 receptors and potassium conductances in physiologically relevant systems. The pharmacology of potassium channels still lacks of great specificity and this surely doesn't permit to exclude possible artefactual results; notwithstanding this limitation, these reports suggest the possibility of an involvement of potassium channels in DSI, which is an extremely interesting hypothesis, worth being further investigated.

\subsection{Other possible targets}

The range of possible targets modulated by cannabinoid receptors is not limited to the voltagedependent ion channels involved in synaptic transmission. Current evidence also supports the 
possibility of a more direct inhibition of the release machinery. Cannabinoids affect external calcium- and action potential-independent transmission in many distinct regions of the CNS; these so called miniature events are classically considered to come from the spontaneous fusion of synaptic vesicles with the presynaptic membrane; they thus represent a sequence of randomly occurring molecular events succesfully leading to transmission.

Cannabinoids potently reduce the frequency (but never the amplitude) of TTX-insensitive miniature GABAergic currents (mIPSCs) in rat PAG (Vaughan et al., 2000), rat Rostral Ventromedial Medulla neurons (Vaughan et al., 1999), mouse Nucleus Accumbens (Manzoni et Bockaert, 2001), rat Superficial Medullary Dorsal Horn (Jennings et al., 2001) and cultured hippocampal neurons (Irving et al., 2000) and, for glutamatergic mEPSCs, in the mouse Nucleus Accumbens (Robbe et al., 2001), in hippocampal CA1 pyramidal cells (Misner and Sullivan, 1999) and, in the cerebellum, at the parallel fibers to Purkinje cell synapse (Levenes et al., 1998; but see later).

Nonetheless, it should be underlined that not in all the systems where cannabinoids inhibit action potential dependent transmission, this inhibition is accompanied by a reduction in miniature event frequency. For example, GABAergic miniature currents are not inhibited in the Amygdala (Katona et al., 2001) nor, interestingly, at the same GABAergic synapses onto hippocampal CA1 pyramidal cells which express DSI of evoked currents.

Finally, glutamatergic systems where minis are not influenced by cannabinoids are cultured hippocampal neurons (Sullivan, 1999) and, in contrast to the report of Levenes et al. (1998), the parallel fiber to Purkinje cell synapses (Takahashi and Linden, 2000). In this case, the discrepancy might be produced by differences in the analysis methods, seen the difficult detectability of these events, which are extremely small and hardly distinguishable from the basal noise.

What could be the possible mechanisms of action of cannabinoids on the GABAergic release machinery in the cerebellum?

It must be first remarked that mIPSCs from basket/stellate onto Purkinje cells are strongly modulated by agents interfering with the cAMP pathway: membrane permeant cAMP analogues and adenylate cyclase activators lead to a strong increase in mIPSC frequency (Llano and Gerschenfeld, 1993; shown to be PKA-dependent in Kondo and Marty, 1997); consistently, neuromodulators like the monoamines noradrenaline (NA) and serotonin, known to increase cAMP-related pathways (in both cases the effect is PKA-dependent: see Kondo and Marty, 1997, ; Mitoma and Konishi, 1999), have remarkable potentiating effects on mini frequency. From these data, it 
seems clear that increased cAMP levels lead to a rise in release probability; on the other hand, other G-protein-coupled receptors like mGluR2 (Prezeau et al., 1992), GABAb (Kaupmann et al., 1997) as well as CB1Rs (Matsuda et al., 1990) are negatively coupled to adenylate cyclase in expression systems and strongly inhibit miniature release (see Glitsch et al., 1996 for mGluR2 and Than and Szabo, 2002 for GABAb), suggesting that also this type of depression may take place through cAMP.

However, there is no evidence indicating that adenylate cyclase is constitutively active in control conditions in presynaptic GABAergic terminals. Kondo and Marty (1997) reported a higher, not a lower, mini frequency in slices pre-treated with an adenylate cyclase inhibitor with respect to control slices, while Glitsch ( $\mathrm{PhD}$ thesis, 1998) reports no significant effects of the same agent in the same preparation. In addition, Kondo and Marty found no difference in mIPSC frequency between control and staurosporine-treated slices (staurosporine is an unspecific protein kinase inhibitor), suggesting that Protein Kinase A is not constitutively active. Nonetheless, these can be considered as preliminary data of a line of research which has not been pursued further and, in my view, they still leave this important question open.

Establishing that the resting cAMP concentration suffices to upmodulate pathways related to synaptic transmission and firing frequency (see for example how noradrenaline upregulates Ih in cAMP-dependent way to increase basket cell spike rate in the cerebellum: Saitow and Konishi, 2000) would automatically candidate cAMP-related pathways as targets of inhibitory actions by G-protein coupled receptors.

cAMP could thus have complex effects on our synapses, with multiple modulatory pathways involved. As mentioned, the cloned CB1R is negatively coupled to adenylate cyclase and so its action on miniature release perfectly fits into this scheme; not surprisingly, the action of cannabinoids on the brain has long been associated with modulations in cAMP levels (Martin, 1988).

One possible mechanism of transmission control by cAMP-mediated processes is a direct modification of proteins involved in vesicle fusion. There is already strong evidence in this direction: for example in the Drosophila Neuromuscular Junction (NMJ), an external calcium-independent but PKA-dependent effect on miniature transmission depends on the presence of functional synaptobrevin (Yoshihara et al., 2000), which is one of the SNARE proteins involved in vesicular fusion. Furthermore, SNAP-25, another of the protein forming the SNARE complex, can be phosphorylated by PKA (Risinger and Bennet, 1999) as well as the synaptic vesicle proteins 
synapsin I and II (see for example, Hosaka et al., 1999; synapsins have long been thought to be involved in synaptic vesicle movement along actin filaments) and rabphilin-3A (Fykse et al., 1995). Moreover, it has recently been shown that small guanine nucleotide exchange factors have in their structure cAMP-binding domains through which this cyclic nucleotide can directly modulate their function (deRooij et al., 1998; Kawasaki et al., 1998), thus raising the possibility that synaptic proteins could have similar domains too. The change in release probability caused by cAMP-dependent processes could also be due to more subtle effects, like a change in the number of vesicles in a high release probability state (like in the calyx of Held: Sakaba and Neher, 2001 or at Drosophila NMJ: Kuromi and Kidokoro, 2000) through facilitated recruitment from other reserve pools in synaptic terminals.

Particularly interesting is, finally, the potentiating effect of the upregulated hyperpolarizationactivated Ih on synaptic transmission at the crayfish neuromuscular junction (Beaumont and Zucker, 2000). Here, serotonin increases the levels of cAMP in nerve terminals, in this way coming to modulate the Ih conductance and, finally, synaptic strength; this process cannot be explained only by the depolarization of the synaptic terminals produced by a shift in the Ih activation curve, which is often the effect of neuromodulators pathways on this conductance (McCormick and Pape, 1990; Maccaferri and McBain, 1996 just for two out of several examples). What is proposed is a direct action on the release machinery and/or a recruitment of synaptic vesicles into the readily releasable pool.

The important message is that regulation of voltage-dependent conductances can also induce changes in synaptic action which are not strictly dependent on their influence on the cell membrane voltage. 


\section{Chapter 4}

\section{Materials and Methods}

All the experiments, both electrophysiological and morphological ones, have been performed on 11-15 days old rats.

\subsection{Preparation for electrophysiology}

Animals were anesthetized with Metofane (Janssen-Cilag, Neuss, Germany); after decapitation, the cerebellar vermis was fastly removed and placed into bicarbonate-buffered saline (BBS) at $4-5^{\circ} \mathrm{C}$ for 5-10 minutes; colder cutting solutions led to a massive lost of interneurons in the lower third of the molecular layer and to a general worsening of the slice conditions. 180 $\mu \mathrm{m}$-thick slices were then cut in the parasagittal orientation with a Leica VT1000S vibratome (Nussloch, Germany) and left for other 5-10 min in BBS at room temperature before being moved to the same medium at $34^{\circ} \mathrm{C}$ for one hour and then back to BBS at room temperature for the rest of the experimental day. This protocol optimized slice conditions for paired recordings, especially by increasing the percentage of interneurons surviving the cutting procedure. When recording uniquely from Purkinje cells, slices were incubated only in the warmer BBS. Slices were then transferred to the recording chamber and perfused with BBS at a rate of 1-1.5 ml / min at room temperature. BBS contained (in $\mathrm{mM}$ ): $\mathrm{NaCl} 125, \mathrm{KCl} 2.5, \mathrm{CaCl} 2, \mathrm{MgCl}_{2} 1$, $\mathrm{NaH}_{2} \mathrm{PO}_{4} 1.25, \mathrm{NaHCO}_{3} 26$, glucose $10, \mathrm{pH} 7.4$ equilibrated by continuously bubbling with $95 \% \mathrm{O}_{2}, 5 \% \mathrm{CO}_{2}$ (All these chemicals were from Sigma-Aldrich, Taufkirchen, Germany). Except where indicated, GABAergic currents were pharmacologically isolated with NBQX (2 $10 \mu \mathrm{M}$ ) and APV (20 - $50 \mu \mathrm{M}$; both from Tocris Cookson, Bristol, UK) in the bath BBS. To record TTX-insensitive miniature IPSCs (mIPSCs), TTX (200 or 500 nM; Sigma-Aldrich) was 
directly added to the BBS.

Purkinje cells and interneurons were identified using an upright microscope (Axioscope, Zeiss, Jena, Germany) with differential interference contrast optics, a $63 \times, 0.9$ NA water immersion objective and a 0.63 NA condenser.

In cerebellar sagittal slices, Purkinje cell identification was rendered unequivocal by their characteristic position and the dimensions of their soma. Basket cells in the lower third of the molecular layer were rare in our slices; they were the most sensitive cell type to the cutting stress, but, once present and healthy, they could easily be distinguished from Bergmann glial cells for their bigger soma. Higher basket and stellate cell soma have smaller diameter and, at the developmental stage of our animals, can be easily confused with migrating granule cells. In this case, electrophysiological criteria such as the ability of firing spikes were used.

\subsection{Electrophysiological recordings}

Experiments were performed with either a single or a double EPC-9 amplifier (HEKA Elektronic, Darmstadt, Germany) controlled by the Pulse software (HEKA Elektronic).

Recording pipettes were pulled from borosilicate glass capillaries (Purkinje cells: 2 - $3 \mathrm{M} \Omega$ in chloride-based internal solution, 3.2 - 4.2 $\mathrm{M} \Omega$ in gluconate-based solution; interneurons: 8-12 $\mathrm{M} \Omega$ for paired recordings; 4 - $7 \mathrm{M} \Omega$ for calcium imaging) using a List L/M-3P-A vertical puller (List Medical, Darmstadt, Germany). Purkinje cells were recorded from in the standard wholecell, voltage-clamp configuration with several intracellular solutions; most of the experiments have been conducted with cesium-based solutions, to increase the Purkinje cell input resistance and better clamp the cell voltage. Different types and concentrations of anions were used (in $\mathrm{mM})$ :

- SOLUTION 1:

CsCl 150, $\mathrm{MgCl}_{2}$ 4.6, $\mathrm{CaCl}_{2}$ 0.1, Hepes 10, EGTA 1, Na-ATP 4, Na-GTP 0.4.

This solution was used to record spontaneous IPSCs (sIPSCs) and mIPSCs for the results of chapter 5. At the Purkinje cell holding potential of $-70 \mathrm{mV}$, the calculated $-10 \mathrm{mV}$ chloride reversal potential gave rise to inward synaptic currents, greatly facilitating event detection.

For evoked synaptic currents in paired recordings, either the previous solution was used or two other cesium-gluconate based ones, with different chloride concentrations; a serious problem 
using intracellular solutions containing high chloride concentrations was given by the instability of the access resistance, which constantly and steadily tended to worsen with time in whole-cell. In this sense, gluconate-based solutions offered a great advantage, by increasing the stability of the experiment; this was counterbalanced by the smaller amplitude of GABAergic events:

- SOLUTION 2:

CsGluconate 150, $\mathrm{MgCl}_{2}$ 4.6, $\mathrm{CaCl}_{2}$ 0.1, Hepes 10, EGTA 1, Na-ATP 4, Na-GTP 0.4

- SOLUTION 3:

CsGluconate 130, $\mathrm{CsCl} 20, \mathrm{MgCl}_{2}$ 4.6, $\mathrm{CaCl}_{2}$ 0.1, Hepes 10, EGTA 1, Na-ATP 4, NaGTP 0.4

The following solution was used for Purkinje cells only for the few experiments described in Fig.5.8:

- SOLUTION 4:

KGluconate 150, $\mathrm{MgCl}_{2}$ 4.6, $\mathrm{CaCl}_{2}$ 0.1, Hepes 10, EGTA 1, Na-ATP 4, Na-GTP 0.4

Presynaptic interneurons were recorded from in the perforated-patch configuration using either SOLUTION 4 or the following, low chloride-containing one:

\section{- SOLUTION 5:}

KGluconate 155, $\mathrm{MgCl}_{2}$ 1, $\mathrm{CaCl}_{2}$ 0.1, Hepes 10, EGTA 1, Na-ATP 4, Na-GTP 0.4

To record evoked IPSCs (eIPSCs) in paired recordings, Amphotericin B was used to get the perforated patch configuration on presynaptic interneurons; aliquots of AmphB were freshly prepared in DMSO at a saturating concentration of $1 \mathrm{mg} / 15 \mu \mathrm{l} ; 1.5-2 \mu \mathrm{l}$ were then dissolved in $0.5 \mathrm{ml}$ intracellular solution and solubilization was achieved by vortexing and shortly sonicating. DMSO final concentration in the intracellular solution was, thus, comprised between 1:250 or 1:350; this content has never provided problems in obtaining stable seals and durable recordings. The solution was then kept at room temperature in the darkness during experiments. Pipette tips were filled by immersion into the AmphB-free solution and then backfilled with AmphB.

Several gigaohm seals could be achieved with this procedure; nevertheless, correct presynaptic stimulation couls also be obtained with seals in the order of hundreds of megaohms. Typically, after maximum $5 \mathrm{~min}$ from sealing enough electrical access into the cell could be achieved to induce unclamped presynaptic action potentials by depolarizations from a holding potential of 
$-60 /-70 \mathrm{mV}$ to between 0 and $+20 \mathrm{mV}$ for $0-10 \mathrm{~ms}$ in voltage-clamp; access into interneurons normally improved with time until final, steady values of 30 - $40 \mathrm{M} \Omega$ for the best experiments. Presynaptic series resistance was never compensated. In many experiments, leak currents were subtracted from the raw trace by using the $\mathrm{P} / \mathrm{N}$ algorithm directly controled by the Pulse software.

Alternatively, in current clamp (CC), positive currents of different amplitudes were injected into the cell to induce single action potentials. In paired pulse experiments, the same stimulation protocol in $\mathrm{VC}$, as well as in $\mathrm{CC}$, was repeated at the intervals indicated for each case. Interneurons were stimulated at $0.2 \mathrm{~Hz}$. Two different DSI induction protocols were used: Purkinje cells were depolarized either 8 times for $100 \mathrm{~ms}$ to $0 \mathrm{mV}$ at $1 \mathrm{hz}$ or once to $0 \mathrm{mV}$ for $1 \mathrm{~s}$. Both protocols led to maximal DSI induction, which has been shown in our system to be strictly dependent upon the increase in calcium concentration (Glitsch et al., 2000) and, so, on the depolarization protocol.

For the series of experiments studying the effect of Purkinje cell depolarizations on firing frequency, interneurons were recorded in current clamp through the perforated patch configuration; to obtain a stable firing pattern, small currents in the order of a few picoamperes had sometimes to be injected although, in most cases, interneurons were spontaneously active at $0 \mathrm{pA}$ current.

\subsection{Electrophysiological data analysis}

All the analysis has been performed using home-made routines within the IGOR programming environment (Wavemetrics, Lake Oswego, USA); for sIPSC and mIPSC analysis, these were kindly provided by Dr. Christophe Pouzat.

\subsubsection{Analysis of the DSI of mIPSCs, of sIPSCs and of interneuron action potentials}

mIPSCs and sIPSCs were continuously recorded during $30 \mathrm{~s}-1$ min-long pre- and 1 min-long post-DSI periods. Individual IPSCs were detected by analysing the first and second derivatives

of previously smoothed current traces. Mini peaks corresponded to points of null first derivative and of positive second derivative. The amplitude of each event was given by the difference between the peak amplitude and the amplitude of the point where the first derivative of the smoothed trace altered its sign, as found by running backwards on the trace, starting at the peak. An event was accepted if its amplitude was higher than a threshold set for each experiment, 
according to the noise level (normally in the range from 10 to $20 \mathrm{pA}$ ). Because of the large difference between the IPSC risetime (20\% - 80\% risetime: 1 to $2 \mathrm{~ms}$ ) and decay time ( $\tau$ on the order of $10-15 \mathrm{~ms}$ ), the detecting method led to a reliable detection and to a negligible error in the measure of amplitudes of overlapping events. Visual inspection of the entire traces was always performed to ensure that the parameters of the detection routine were optimally set for each experiment, and to verify that the analysis program did not produce significant errors. Time was then divided into bins (duration, $2 \mathrm{~s}$ ) and the frequency, the average amplitude and the cumulative amplitude (given by the sum of the amplitudes of all the events falling into a bin) of the IPSCs in each bin were calculated to give the time course of these parameters. After normalization with respect to the average pre-DSI values, control and test (in the case of pharmacological treatments) DSIs were separately pooled together. DSI was calculated as the reduction in the average (over the bin values in the chosen time window) frequency, amplitude and cumulative amplitude over a period from 2 to $12 \mathrm{~s}$ after Purkinje cell depolarization with respect to the pre-DSI period.

The same program was used also to detect presynaptic action potentials recorded from current clamped interneurons; in this case the points of minima were represented by the action potential peaks, after changing the sign of the voltage trace to increase detectability. The threshold was changed accordingly (range: 10 to $20 \mathrm{mV}$ ). Organization of control and test periods into $2 \mathrm{~s}-$ long bins and normalisation procedures for action potential frequencies were the same as for mIPSCs and sIPSCs. The DSI of action potentials was calculated between 4 and $10 \mathrm{~s}$ after the induction protocol, where spike inhibition was maximal.

\subsubsection{Analysis of the DSI of eIPSCs}

The detection algorithm was essentially the same as for mIPSCs and sIPSCs.

The risetime was calculated by finding the couples of sampled points on the rising phase of each evoked current which comprises the $20 \%$ and $80 \%$ of the calculated event amplitude, respectively; each couple of points was then fitted linearly and the occurrence time of the $20 \%$ and $80 \%$ points calculated on the fitting line. The latency was given by the time between the peak of the presynaptic action potential and the $20 \%$ point on the rising phase of the eIPSCs. Each evoked current was fitted with a double exponential starting from the point of peak amplitude; the decay time constant was given by averaging the two exponential time constants weighted for the relative amplitude contribution of the 2 components. 
Evoked currents were recorded during 60 or $90 \mathrm{~s}$ both in the pre-DSI and post-DSI periods. Control and test (for pharmacological treatments) periods consisted of several DSI trials (3-5) each. For each trial, eIPSC amplitudes were normalized with respect to the average amplitude of pre-depolarization eIPSCs. Control and test time courses for an experiment were obtained by pooling together these single normalized control and test DSIs. The extent of this form of DSI $_{\text {IIPSC }}$ was calculated as the percentage reduction in peak amplitude for the average eIPSC obtained from a variable number of eIPSCs immediately following DSI induction, according to the time window for which DSI was measured. Normally, the maximal DSI was calculated from the first 3 post-protocol eIPSCs, which covered the first $10 \mathrm{~s}$ during DSI. Paired pulse ratioes were calculated as the percentage change in the amplitude of the average second eIPSC versus the amplitude of the average first eIPSC for a same sample of evoked currents: at least 30 sweeps before and 30 sweeps after a pharmacological treatment were considered, whereas the sweeps taking place in the first 40s following DSI induction (8 eIPSCs for a $0.2 \mathrm{~Hz}$ stimulation rate) compared with the ones spanning the 60-90s preceding DSI for DSI experiments. Similarily, to compare eIPCS failure rate, latency, risetime and decay time constants before and after DSI, the first post-depolarization $40 \mathrm{~s}$ were considered. This time window was chosen to try and reduce the variability of these measures originating from the extreme intrinsic variability of synaptic transmission in our system.

Synaptic parameters like the variance over mean ratio ( $/ \mathrm{m}$ ratio) and the inverse of the squared coefficient of variation $\left(\mathrm{CV}^{-2}\right)$ were calculated for an eIPSC sample of at least 30 sweeps in control and test periods, for pharmacological experiments. In order to limit the effect of the fast time course of recovery of DSI on the variability of the evoked currents, in the case of DSI experiments groups of three consecutive eIPSCs were produced in the control and test periods, average amplitudes and $\mathrm{CV}^{-2}$ were calculated for each group and the values for the first two triplets in the test period were normalized to the average control values. In this way, for each paired recording two points were obtained, corresponding to two different intervals of the test period, in which DSI could be considered stationary.

Levels of significance were set at 0.05 and calculated using the the Mann-Whitney and/or Wilcoxon signed-ranks (where control and test periods could be compared in single experiments) tests. 


\subsection{Fluorometric calcium imaging experiments and their analysis}

Action potential-dependent calcium transients were recorded in cerebellar interneuron axons as described earlier (Forti et al., 2000). All the experiments were performed with a single EPC-9 amplifier (HEKA) using the following intracellular solution:

\section{- SOLUTION 6:}

KGluconate $155, \mathrm{KCl} 10, \mathrm{MgCl}_{2}$ 2.4, Hepes 10, Na-ATP 2.4, Na-GTP 0.4, whose osmolarity (around 326mOsm) was corrected by adding the fluorescent dye Oregon Green 488 BAPTA-1 (OG1; $250 \mu$ M; Molecular Probes, Eugene, Or, USA) dissolved in distilled water.

Fluorescence images were obtained using an excitation-acquisition system from T.I.L.L. Photonics (Planegg, Germany). Light coming from a 75 W Xenon lamp was focused onto a scanning monochromator set at $488 \mathrm{~nm}$ and coupled to the microscope by a quartz fibre and a lens; a dichroic mirror and high-pass emission filter centred respectively at 505 and $507 \mathrm{~nm}$ were placed in the light pathway. Images were acquired by a Peltier-cooled PCO SensiCam camera connected to a 12 bit A/D converter with a maximal resolution of $640 \times 480$ pixels (pixel size is $0.25 \mu \mathrm{m}$ after a $63 \times$ magnification).

Interneurons were recorded in voltage clamp. After allowing 8-12 min from break in to let the dye diffuse into the cell, a few images were taken with a short exposure time $(20-30 \mathrm{~ms})$ to study the morphology of the recorded cell and find an axonal area showing recognizable varicosities.

During recording, series of 24 images with an exposure time of $50 \mathrm{~ms}$ and a resolution of 200 $\times 200$ pixels were taken every 3 to $5 \mathrm{~min}$ to monitor calcium transients. In each series, 4 depolarising steps to $0 \mathrm{mV}$ at $50 \mathrm{~Hz}$ were applied to the cell simultaneously with the 6th image, to induce unclamped action potentials in the axon.

There were clearly recognizable signs of photodamage, which developed with repeated fluorescence acquisition sequences, as follows: a large negative increase of the baseline inward current; an increase in the electrical baseline noise; the appearance of squared inward currents on the repolarization phase of each stimulus pulse; failure of action potentials to elicit fluorescent transients. A thorough quantification, which well applies to this series of experiments too, can be found in Forti et al., 2000. At the onset of one of these signs, experiments were interrupted. 
In control experiments, calcium transients and action currents recorded in voltage clamp decreased with time in whole-cell. Calcium signals showed a biphasic run-down, with a faster component in the first $30 \mathrm{~min}$, followed by a smaller and slower component.

For analysis, 4 background regions and 6 to 10 regions of interest (ROIs) located on axonal structures were chosen. The mean background was subtracted from the fluorescence of each pixel $i$ and the percentage change in calcium at image $n$ was computed as $\Delta F_{i}(n) / F_{i} 0=100\left(F_{i}(n)-\right.$ Fi0)/ $/ F_{i} 0$ where $F_{i} 0$ and $F_{i}(n)$ are respectively the background-subtracted average resting fluorescence and the background-subtracted fluorescence at image n. ROI values were averaged to obtain an estimate of the increase in fluorescence in the axon for each image. The time course for the experiment was then obtained by normalizing the maximal changes in fluorescence for each series by the value of the first series in the experiment. WIN 55,212-2 was applied after 4 control sequences of images, at times ranging from 25 to 43 min after break-in. To pool together the experiments, time was divided into 5 min bins with the moment of WIN application considered as $\mathrm{t}=0$. Values from each experiment falling in a corresponding bin were then averaged and bin values were normalized with respect to the pre-application period. The points in WIN 55,212-22 were corrected for rundown by using a linear extrapolation of the control period $(t<0)$, normally consisting in 4 image series. The time course of fluorescence for control experiments was obtained with the same procedure and $\mathrm{t}=0$ was set at breaking-in into the recorded interneuron.

\subsection{Morphological reconstructions}

The morphology of synaptically connected cells was recovered as follows. During paired recordings, Purkinje cells were filled through the recording pipette with Lucifer Yellow (0.3 $\%$; Sigma). Typically, at the end of a perforated patch recording the cell body of the presynaptic interneurons remained intact after withdrawal of the recording pipette. A second intracellullar pipette was then filled with the lipophilic dye Dil (Molecular Probes) dissolved in methylene chloride ( $0.5 \%$; Sigma). After the formation of a dye crystal on its tip, the pipette was placed in contact with the cell soma and rapidly removed, in order to permit the deposition of the crystal, without damaging the cellular structure. The slices were then fixed for $48 \mathrm{hrs}$ at $4-5 \mathrm{C}$ in $4 \%$ paraformaldehyde (Sigma) in 0.15 M Phosphate Buffered solution (PB) and left in PB for 48 hrs at the same temperature to permit full diffusion of the lipophilic dye. Finally, the slices 
were mounted on glass slides in DAKO (Dako, Carpinteria, Ca, USA) fluorescence mounting medium. Series of images at incremental z-axes positions were taken and superimposed using a Zeiss LSM 410 confocal microscope equipped with two different lasers at $488 \mathrm{~nm}$ and $543 \mathrm{~nm}$ wavelength for Lucifer Yellow and Dil, respectively.

\subsection{Immunohistochemistry}

After rat anesthesia and decapitation, the cerebellar vermis was extracted and fixed in phosphate buffered solution (PB $0.15 \mathrm{M}$ ) with $4 \%$ paraformaldehyde for $1 \mathrm{hr} .80 \mu \mathrm{m}$ thick parasagittal slices were then cut and left in the fixative solution for further $3 \mathrm{hr}$. These sections were blocked with bovine serum albumin (BSA; 1\%) in PB (blocking buffer: BB) for $1 \mathrm{hr}$ and, then, incubated in the same solution plus the primary antibody (1:500) in 2 steps; a first incubation for $24 \mathrm{hr}$ at $4-5^{\circ} \mathrm{C}$ with Triton X-100 (0.4\%), followed by another $24 \mathrm{hr}$-long period at the same temperature without the permeabilizing agent in the BB. An affinity purified primary antibody against the CB1R C-terminal (Hajos et al., 2000), whose specificity was thoroughly tested in the laboratory of origin, was used. The antibody was provided by Dr. Ken Mackie of the University of Washington, Seattle, USA.

The incubation with a Cy3-conjugated goat anti-rabbit secondary antibody (1:100; Jackson ImmunoResearch, West Grove, PA, USA) was done for $3 \mathrm{hr}$ at room temperature. Slices were washed in BB and in PB and, finally, mounted on glass slides in DAKO fluorescent mounting medium. Controls consisted in incubations with only the secondary antibody.

Images were acquired with a Zeiss (Oberkochen, Germany) LSM 410 confocal microscope (excitation light: $543 \mathrm{~nm}$ ).

\section{Phosphate Buffered solution (PB 0.15 M)}

For 1 liter of concentrated solutions of $\mathrm{Na}_{2} \mathrm{HPO}_{4}(0.3 \mathrm{M})$ and of $\mathrm{NaH}_{2} \mathrm{PO}_{4}(0.3 \mathrm{M})$, respectively:

- $\mathrm{Na}_{2} \mathrm{HPO}_{4}: 53.1 \mathrm{~g}$

- $\mathrm{NaH}_{2} \mathrm{PO}_{4}: 41.4 \mathrm{~g}$

From these stocks, 1 liter of final PB can be obtained:

- $0.3 \mathrm{M}$ solution $\mathrm{Na}_{2} \mathrm{HPO}_{4}: 405.0 \mathrm{ml}$ 
- $0.3 \mathrm{M}$ solution $\mathrm{NaH}_{2} \mathrm{PO}_{4}: 95.0 \mathrm{ml}$

brought to volume with $500 \mathrm{ml}$ of water; $\mathrm{pH}$ and osmolarity were corrected to 7.4 and 320 mOsm, respectively. 


\section{Chapter 5}

\section{Results: DSI characterization}

\subsection{DSI of spontaneous TTX-sensitive and of miniature TTX-insensitive IPSCs}

Cerebellar DSI is known to involve both a TTX-insensitive component and a TTX-sensitive component.

I first analysed the characteristics of the DSI of spontaneous IPSCs. sIPSCs were recorded from voltage-clamped Purkinje cells using a high chloride intracellular solution; this strongly improved the process of event detection by maximizing the driving force for this ion through the GABA receptors.

The upper trace in Fig.5.1A illustrates the response of sIPSC to a series of $8 \times 100 \mathrm{~ms}$ depolarizing pulses at $1 \mathrm{~Hz}$. Similarly to the $1 \mathrm{~s}$-long, single depolarization to $0 \mathrm{mV}$ widely used for other DSI experiments described in this thesis, such protocol has been shown to produce a fully saturated DSI in Purkinje cells (Glitsch et al., 2000). The train induced a very strong reduction in SIPSC, which slowly recovered over a time course of about 1 minute. In individual experiments, the peak reduction in cumulated sIPSC amplitude ranged from $81.54 \%$ to 19.48 $\%$ over the ten seconds following the end of the depolarizing train to the Purkinje cells. The middle graph in Fig.5.1A shows the time course of the cumulative amplitude calculated over two s-long bins, for a $30 \mathrm{~s}$ control period and the first post-depolarization minute: sIPSCs recovered to control with a half-time of $37.88 \pm 3.79 \mathrm{~s}$ (value from $\mathrm{n}=13$ experiments).

Average normalised results from such experiments indicate a peak reduction in the sIPSC cumulative amplitude of $64.16 \pm 4.65 \%(n=14)$ during DSI. Reductions in the frequency and in the average amplitude of sIPSCs contributed in equal measure to the decrease of the cumulative 


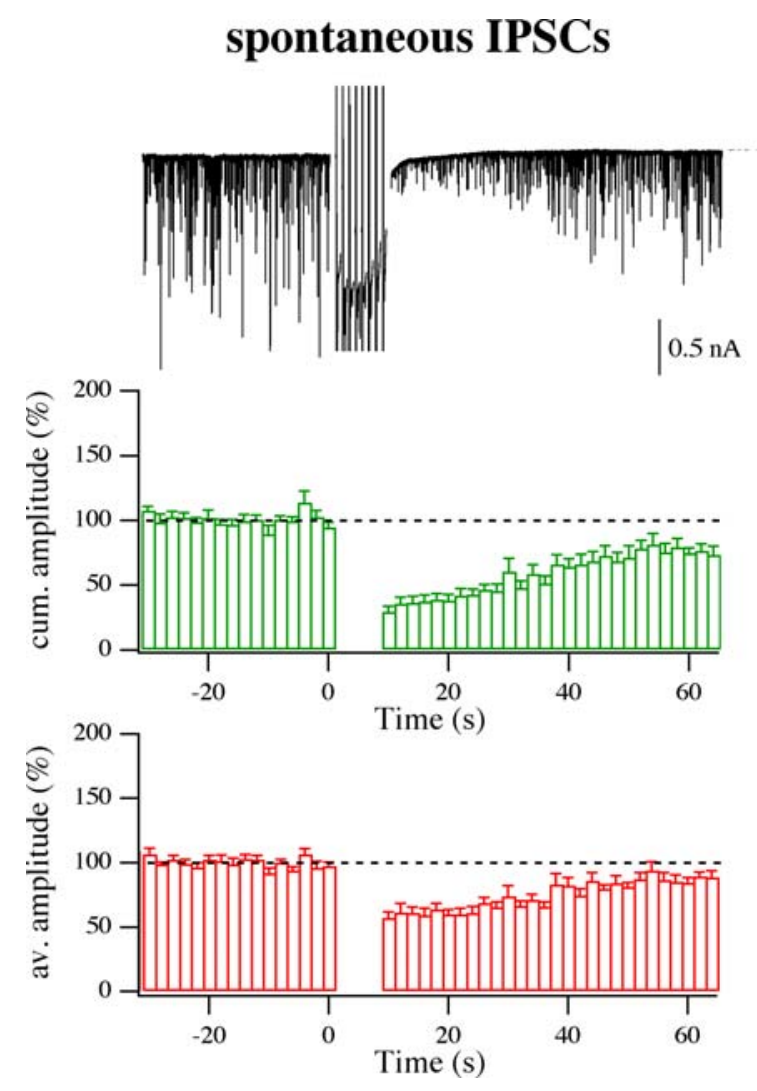

\section{miniature IPSCs}
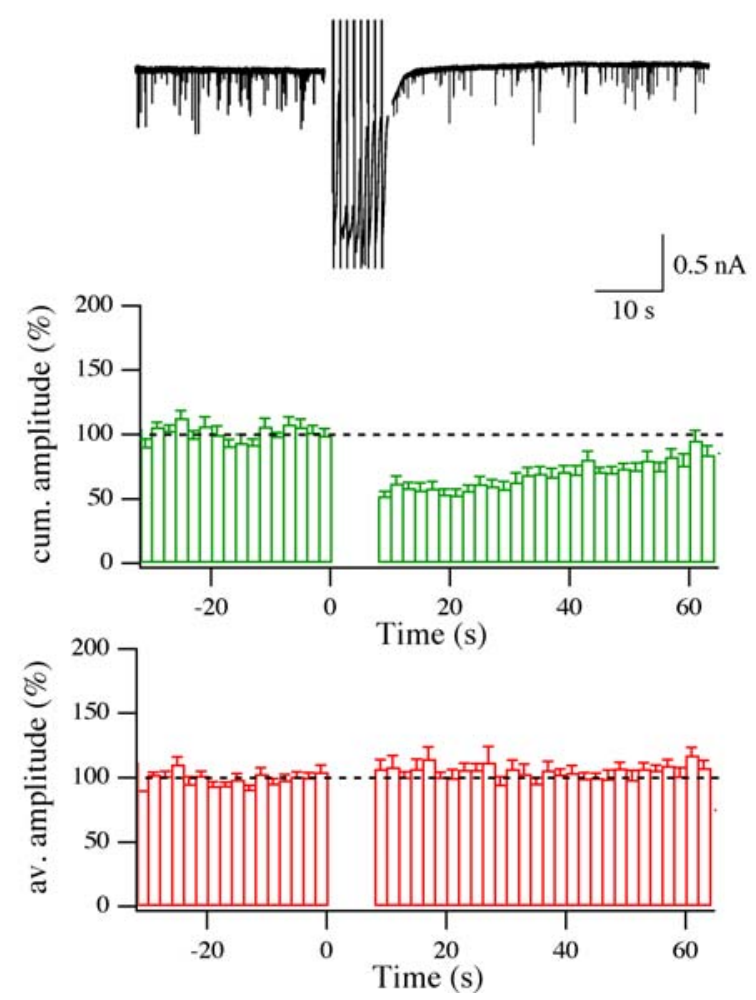

Figure 5.1: DSI of sIPSCs and of mIPSCs. All the data shown in this figure pertain to recordings of synaptic activity from single Purkinje cells. In A (sIPSCs), action potential-induced and external calcium- and action potential-independent synaptic currents contribute to the overall population of recorded IPSCs. The upper trace shows a typical DSI protocol: after a control period, the Purkinje cell is depolarized 8 times for $\mathbf{1 0 0}$ ms to $0 \mathrm{mV}$, at $1 \mathrm{~Hz}$; the trace shows the consequent dramatic inhibition of sIPSCs and, thereafter, the recovery phase of the sIPSCs over a period of $60 \mathrm{~s}$. In this cell, 4 such protocols were averaged, yielding $79.63 \pm$ $0.47 \%$ inhibition in the sIPSCs cumulative amplitude, calculated over the first $10 \mathrm{~s}$ following the end of the pulse train; the decreases in the frequency and in the average amplitude contribute in equal measure to this reduction: $53.57 \pm 4.33 \%$ and $55.19 \pm 4.29 \%$, respectively. The 2 lower graphs show the time course of the DSI of cumulative and average amplitudes for $n=14$ experiments performed with this protocol; as for the single case depicted in A, frequency (not shown) and average amplitude are equally inhibited during DSI and recover back to control levels with the same time course. Fig.1B is organised the same way as Fig.1A, but describing the DSI of miniature IPSCs. In the depicted experiment, the inhibition of the mIPSC cumulative amplitude was $64.75 \pm 3.98 \%$ over 4 DSI trials. In contrast to the sIPSC results, the reduction of mIPSC frequency totally accounts for the DSI of the cumulative amplitude; the lower graph shows that the average amplitude of IPSCs remains totally unaffected by the induction protocol (average graphs from 13 cells). 
amplitude: they amounted to $39.93 \pm 4.74 \%$ and to $41.34 \pm 4.25 \%$, respectively (See the lower graph of Fig.5.1A for the time course of the normalised average amplitude; data concerning the frequency are not shown).

Similar experiments were performed with the presence in the bath of TTX (200nM); a DSI protocol for one of them is shown in the trace up in Fig.1B. The average reduction of the cumulative amplitude for the whole series of experiments was $43.34 \pm 3.48 \%(n=13)$; this value is significantly smaller than that of the synaptic currents in the absence of TTX $(0.005<\mathrm{p}<$ 0.01). In contrast to the situation found without TTX, the entire effect could be accounted for by a change in the event frequency $(45.25 \pm 2.97 \%$, not shown here), while the mean amplitude was unchanged (-5.66 $\pm 4.94 \%$; lower graph of Fig.1B).

This result, which is in agreement with earlier reports (Llano et al., 1991; Glitsch et al., 1996), confirms that DSI does not modify the quantal size as measured from mIPSC recordings, and suggests that the expression of DSI is primarily presynaptic. Here, we note in addition that the half recovery time measured in TTX was $35.12 \pm 3.37 \mathrm{~s}(\mathrm{n}=10)$, which was not different from that observed for TTX-dependent sIPSCs. Thus, even though the extent of DSI differs for TTX-dependent sIPSCs and for TTX-independent mIPSCs, the time course was the same in the two cases.

\subsection{DSI as measured in paired recordings}

sIPSCs must be viewed as a mix of mIPSCs and of action potential-evoked IPSCs. In this sense, it is important to compare the frequency of sIPCSs and of mIPSCs in the two separate series of experiments, whose results in terms of DSI have been described in the previous paragraph: $11.13 \pm 1.43 \mathrm{~Hz}$ (range from $3.46 \mathrm{~Hz}$ to $20.19 \mathrm{~Hz} ; \mathrm{n}=14$ ) and $8.20 \pm 1.39 \mathrm{~Hz}$ (range from 2.73 to $17.66 \mathrm{~Hz} ; \mathrm{n}=15)$, respectively. These values show that action potential-independent IPSCs could contribute in a substantial amount to the overall population of recorded sIPSCs. The finding that sIPSCs were more reduced than mIPSCs during DSI therefore indicated that actionpotential evoked IPSCs were even more strongly inhibited than sIPSCs. This strong inhibition could involve a reduction of the frequency of presynaptic action potentials, of the quantal size of evoked IPSCs or of the probability of neurotransmitter release. To sort out these possibilities, paired recordings were performed between presynaptic interneurons and postsynaptic Purkinje cells. 
Vincent and Marty (1996) described an inverse correlation between the strength of these synaptic connections and the distance in the molecular layer of the presynaptic interneurons; therefore most experiments were performed using interneurons located in the lower two-thirds of the molecular layer as presynaptic partners.

\subsubsection{DSI as measured with presynaptic perforated patch recording: why the perforated patch and not the whole-cell configuration?}

We first performed whole-cell recordings of the presynaptic neurons in order to control the ionic composition and the firing rate of the presynaptic cell. While DSI could be readily obtained under these conditions (not shown), the analysis of the results was severely restricted by the fact that an irreversible decline ("rundown") of the synaptic strength occurred within 10-20 min of presynaptic whole-cell recording (Fig.5.2 shows an experiment in these conditions; notice that presynaptic washout was previously mentioned for the axonal autoreceptor current in Pouzat and Marty, 1999).

To try and perform longer and more stable experiments, we decided to use Amphotericin B in the presynaptic pipette and, thus, to apply the perforated-patch technique to stimulate presynaptic interneurons. This technique turned out to be extremely favourable to record from these cells; enough access to elicit unclamped action potentials could be obtained a few minutes after sealing and, most importantly, evoked currents from postsynaptic Purkinje cells could be recorded without significant run-down for long periods (up to more than $1 \mathrm{hr}$ ), as shown in Fig.5.3.

These results suggest that a diffusible, water soluble substance was responsible for the washout observed with conventional presynaptic whole-cell recording. They further opened the way to a quantitative study of DSI in paired recordings. For this reason, the remaining part of this chapter presents results obtained with the perforated patch technique.

Under these conditions, voltage pulses to $0 \mathrm{mV}$ were applied presynaptically at $0.2 \mathrm{~Hz}$, giving rise to an unclamped action potential and to an evoked IPSC in the corresponding, postsynaptic Purkinje cell (see the white traces in Fig.5.4 or the control traces on the upper left of Fig.5.5). Evoked IPSCs typically were in the nA range: the average amplitude of the synaptic connections was $1001.97 \pm 251.62 \mathrm{pA}(\mathrm{n}=22$ pairs with a postsynaptic chloride-based solution), calculated for each experiment from at least 30 eIPSCs. eIPSCs fluctuated markedly between trials: the variance over mean ratio and the inverse of the squared coefficient of variation were 


\section{whole-cell configuration}
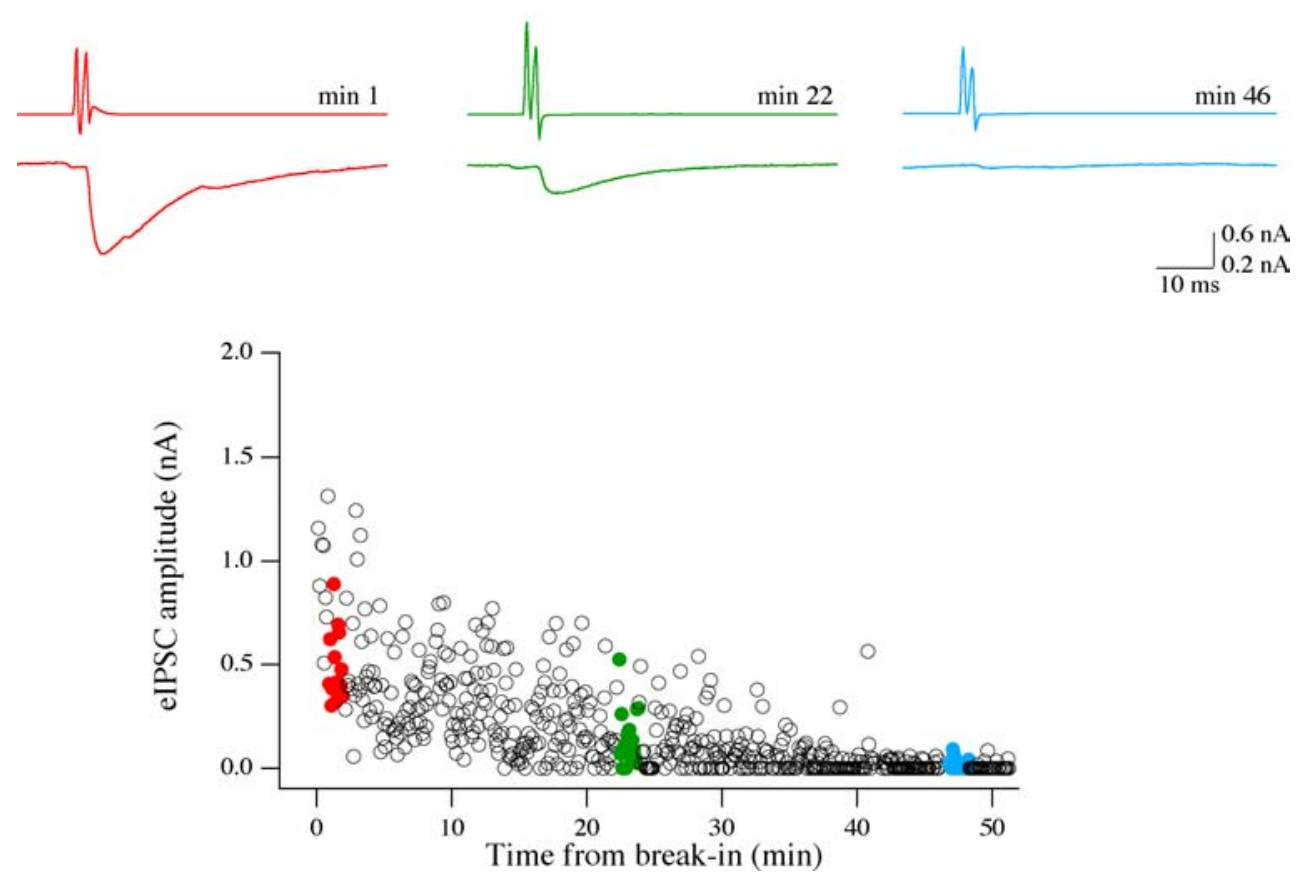

Figure 5.2: The presynaptic whole-cell configuration induced an irreversible run-down of the evoked synaptic currents. This figure shows a typical paired recording, in which the presynaptic interneuron was voltage clamped in the whole-cell configuration with a KGluconate-based solution. It can be noticed that the amplitude of the evoked current in the postsynaptic Purkinje cell slowly decreased with time of presynaptic dialysis. The run-down was irreversible and appeared to depend on the presynaptic access resistance. The traces are averaged currents of the evoked sIPSCs, whose amplitudes are represented on the time plot by filled circles of the corresponding color. This series of experiments suggests that a fundamental, soluble component was washed during the experiment, thus leading to the disappearance of the synaptic connection. The presynaptic interneuron was stimulated at $0.2 \mathrm{~Hz}$. Time zero was set at the moment of the rupture of the presynaptic seal. 


\section{perforated-patch configuration}
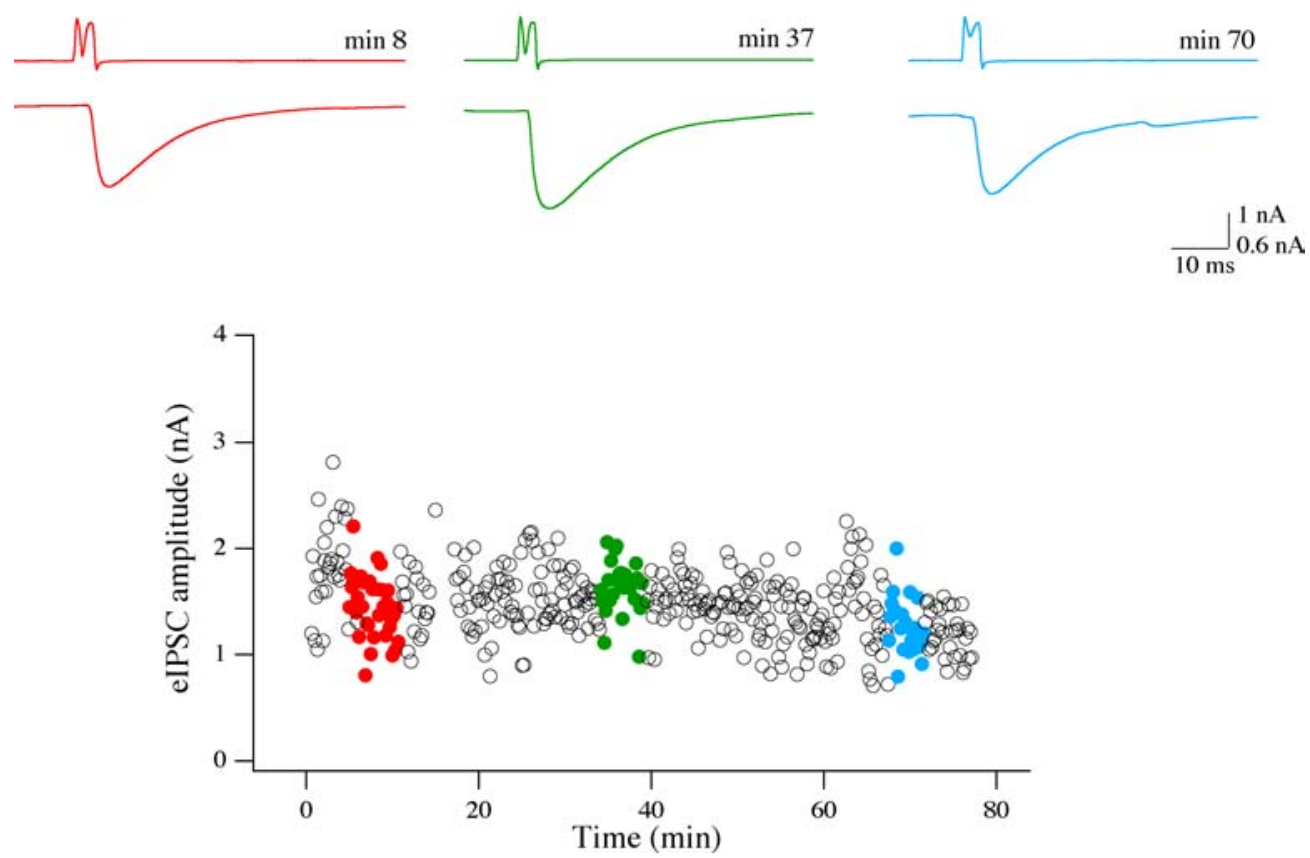

Figure 5.3: Presynaptic perforated patch prevented wash-out of evoked currents and allowed long and stable recording conditions. This figure is organised in the same way as Fig.5.2: the whole experiment is shown in the lower time plot, where each symbol represents the amplitude of 1 evoked current; filled, colored symbols represent the eIPSCs which have been averaged, in parallel with the corresponding presynaptic currents, to give the traces represented above. In this case, time zero was when the presynaptic access was sufficient to trigger an unclamped action potential with a depolarizing pulse. Typically, in most experiments this happened within the first 5 minutes after establishing the seal; the series resistance slowly and continuously improved during the recording, down to minimum values in the order of $30 \mathrm{M} \Omega$. 
$80.17 \pm 12.40 \mathrm{pA}$ and $12.15 \pm 1.99(\mathrm{n}=22)$, repectively.

In a fraction of the experiments, the morphology of the pre- and postsynaptic cells was recovered. Fig.5.4 illustrates a typical example of a low stellate cell synapsing onto a Purkinje cell, with sample pre and postsynaptic currents depicted in white in the lower right corner; connected pairs typically displayed extensive and/or more than a single area of potential contacts, suggesting the presence of multiple release sites. The choice which has been taken to look for presynaptic interneurons located in the lower two-thirds of the molecular layer led to putative synaptic contacts which, in none of the cases examined $(n=8)$, were ever situated on higher order dendritic arborizations. In only very few cases, the morphology of low basket cells were recovered: confirming morphological data on symmetrical synapses in this system at young ages, no axon collaterals forming structures resembling basket cell pinceaux were detected. Low basket cells normally displayed several and extended points of contacts with Purkinje cell bodies and with the primary dendrite, on average more than the contacts typical stellate cells have been found to establish. The bias in the choice of the presynaptic cells, in order to maximise the chances of retrieving strong connections explains the differences in average eIPSC amplitudes between this work and a previous study performed in the same lab (see Vincent and Marty, 1996).

\subsubsection{Purkinje cell depolarizations induce DSI of eIPSCs}

A typical DSI experiment is illustrated in Fig.5.5 and Fig.5.6. After a series of control eIPSCs (normally 18 covering a 90 s-long period), presynaptic stimulations were interrupted and the Purkinje cell was depolarized to induce DSI ( $1 \mathrm{~s}$ pulse to $0 \mathrm{mV}$ ). Shortly thereafter, presynaptic cell stimulations were resumed. During DSI, presynaptic currents were unchanged (Fig.5.5, traces in the upper central inset beneath the schematic description of the depolarization protocol to the Purkinje cell); on the contrary, the evoked IPSCs were markedly reduced from the first post-induction protocol train on (Fig.5.5, compare the upper postsynaptic traces in control, left, versus during DSI, right). The lower graph of Fig.5.5 shows the time course for the same DSI trial as for the traces in the upper part of the figure: each symbol represents the amplitude of one evoked current, red circles represent the $90 \mathrm{~s}$ following DSI induction. It can be noticed that the mean amplitude of evoked IPSCs recovered to values similar to control within 1 min.

Fig.5.6A shows the entire time course of the evoked IPSCs for the same experiment: several 


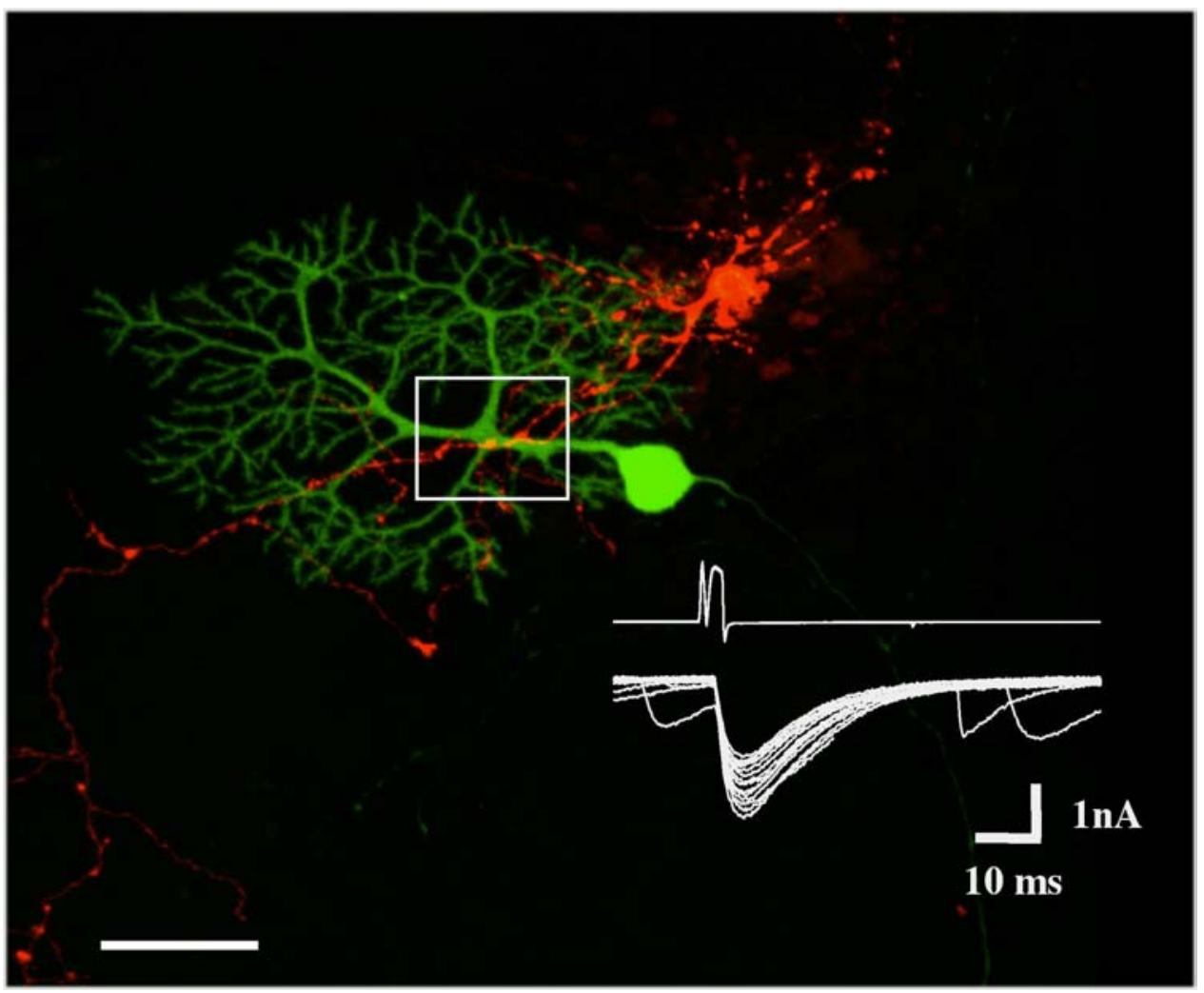

Figure 5.4: Morphological reconstruction of a synaptically connected stellate/Purkinje cell pair. This figure has been obtained by digitally superimposing 60 consecutive pictures, taken with a confocal microscope at $500 \mathrm{~nm}$ increments on the vertical axis. This picture shows the typical morphology of the cell pairs used throughout this thesis work; the presynaptic cell is a low stellate (red; Dil staining), which synapses onto the proximal dendrites of a Purkinje cell (green; Lucifer yellow staining). There are usually several putative synaptic points of contacts: the white box designates an area of wide superposition of the cellular membranes with 2 presynaptic sweelings. Morphologically, they closely resemble the varicosities where calcium signals have been described to originate in response to trains of somatically induced action potentials (Forti et al., 2000). These so called "hot spots" are likely to represent presynaptic terminals. Bar $=\mathbf{3 0} \mu \mathrm{m}$. 

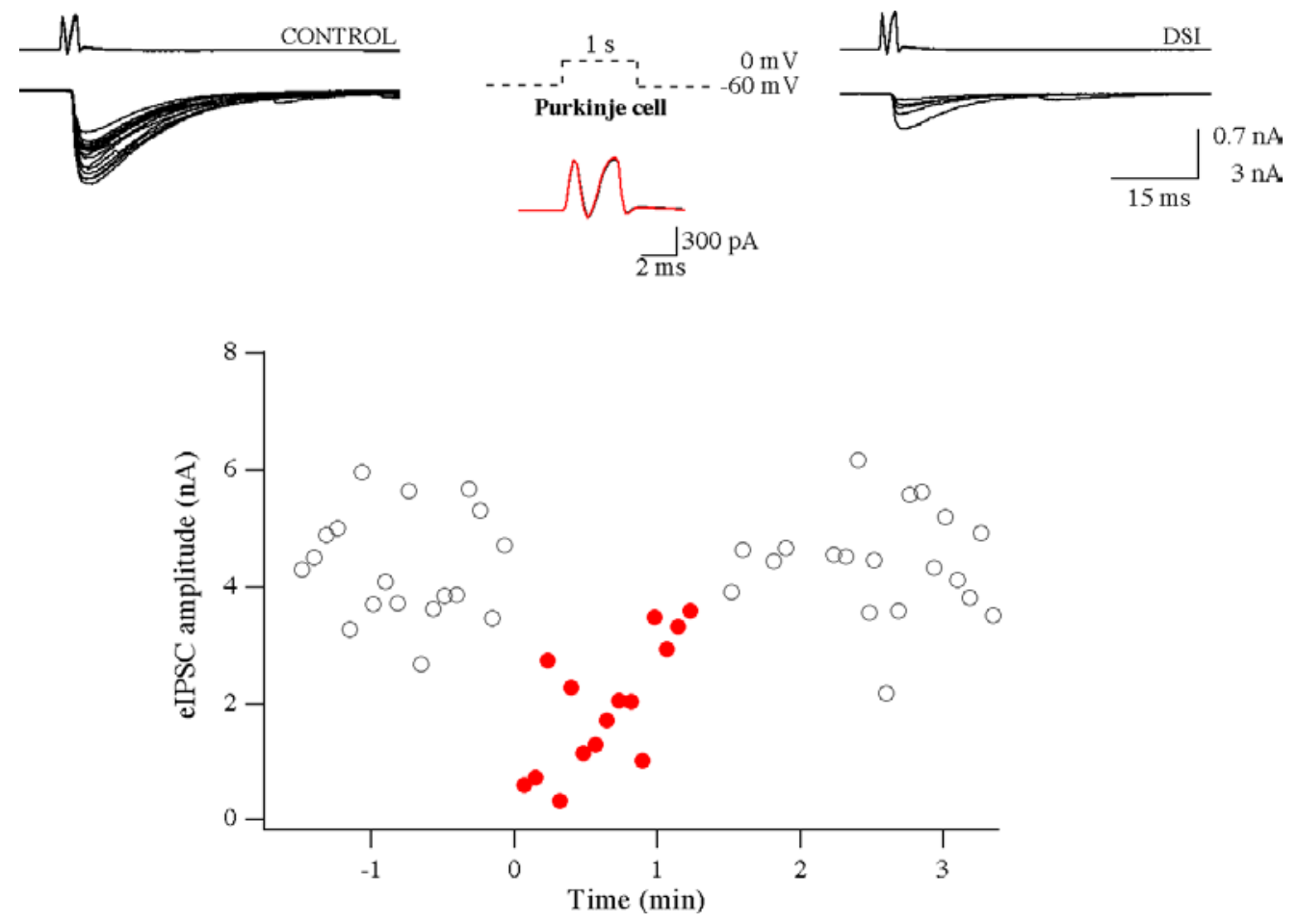

Figure 5.5: DSI can be evoked in paired recordings. This figure shows a DSI trial for a paired recording. DSI is induced by depolarizing the Purkinje cell once to $0 \mathrm{mV}$ for $1 \mathrm{~s}$. In the upper part of the figure, on the left single evoked currents recorded in the Purkinje cell are shown for the control period with the corresponding presynaptic traces; on the right, 5 eIPSCs after the induction protocol are shown; notice the dramatic decrease in the amplitude of the currents. In the middle inset, the induction protocol is schematically drawn and, below, average presynaptic currents are shown superimposed for the control (black) and the test (red) period; no change in presynaptic currents related to the unclamped action potential are detectable at the somatic level. Below, the time course for this DSI trial is shown. Each circle is the amplitude of an evoked current; red, filled ones represent the first 1.5 min during DSI. 

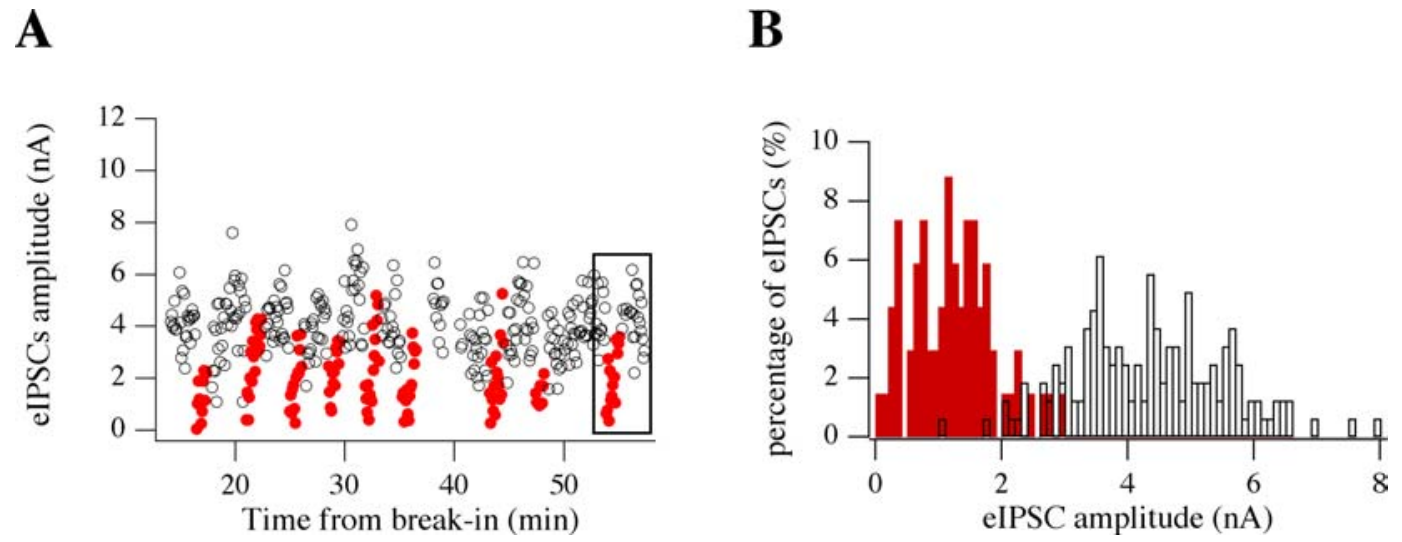

Figure 5.6: This figure illustrates other general features for the experiment of Fig.5.5; in A, the time course of the experiment is shown. Each series of filled red dots represents the $90 \mathrm{~s}$ following a DSI induction protocol; the squared box highlights the DSI trial used for the previous figure. Notice that DSI can be reliably induced for a period of over $\mathbf{4 0}$ minutes, correspondingly with the stability of the strength of this synaptic connection. $\mathrm{B}$, the bar (100 pA bins) histograms shows the density distribution of control eIPSC amplitudes (white bars) and of test eIPSCs (red bars) for the pooled DSI trials of this experiment; the $90 \mathrm{~s}$ preceding DSI were chosen for each depolarization as control (corresponding to 18 eIPSCs, at $0.2 \mathrm{~Hz}$ ) and the following $35 \mathrm{~s}$ as test (8 eIPSCs); notice the clear difference between the two distributions. This implies that action potential failures cannot account for the whole depression seen in DSI and that a mechanism interefering directly with synaptic release must be involved. In this experiment, DSI amounted to a $69.73 \%$ inhibition of the evoked transmission. Consistently with the strength of this connection (control eIPSCs were in the order of several nAmperes), no failures were detected either in control or during DSI. 
DSI trials were performed and DSI could be reliably induced throughout the experiment; red circles always represent the first 1.5 min during DSI. The box points out the single DSI trial depicted in Fig.5.5. Taking advantage from the stationarity in time of the amplitude of evoked IPSCs in the control periods, Fig.5.6B depicts the histogram of the eIPSC amplitudes in the control (open bars) and in the first $35 \mathrm{~s}$ (corresponding to the first 8 eIPSCs) after DSI induction for the pooled DSI trials performed in this experiment. Considering this time window, DSI for this pair amounted to a $69.73 \%$ inhibition of the evoked currents. Even though both histograms are quite broad, reflecting a substantial range for amplitude fluctuations in each condition, there was very little overlap between the two distributions. This excludes that DSI would work simply by inducing blocks of action potential propagation, because if this were the case, the distribution of non-failure events would be the same during DSI and in the control period.

\subsubsection{Reliability of induction and time course of DSI}

The reliability of induction and the time course of DSI shown for the exemplar pair in the 2 previous figures were confirmed when all the pairs, in which DSI was studied with this protocol, were pooled together.

Fig.5.7A shows the value of DSI as a function of the time in the whole-cell configuration for the postsynaptic Purkinje cells. Each DSI trials for each of the $n=17$ performed paired recordings were considered singularly, according to the time of induction from the rupture of the pipette seal on the Purkinje membrane: time was binned in 5 min-long periods and DSI values falling in the corresponding bin averaged. If more than 1 DSI trial for a single pair were falling into a same bin, values were averaged in such a way to have only one value per experiment per bin. As DSI values, the percentage reductions over control of the eIPSC amplitudes in the postdepolarization $35 \mathrm{~s}$ were considered for this analysis. No washout of DSI was visible: DSI remained constant up to $90 \mathrm{~min}$ after the whole-cell configuration was established. As an example, the values of DSI between 5 and $10 \min (55.76 \pm 7.16 \%, \mathrm{n}=11$ DSI trials $)$ and between 50 and $55 \min (61.99 \pm 6.15 \%, \mathrm{n}=13$ DSI trials) after breaking into Purkinje cells are not statistically different $(\mathrm{p}<0.05)$.

Fig.5.7C shows the average time course of DSI from 17 paired recordings. The maximal DSI (calculated over the first 3 eIPSCs after the DSI induction protocol) was $68.81 \pm 4.65 \%$, with values ranging from $23.17 \%$ to $94.35 \%$; In the hippocampus, DSI results are extremely variable in paired recordings: some pairs have essentially $100 \%$ DSI, while others have a negligible DSI 
level (Wilson et al., 2001); this is likely to reflect the different sensitivity to cannabinoids of distinct interneurons subtypes (Katona et al., 1999 ; Tsou et al., 1999). In sharp contrast to this situation, we found that in our preparation, the distribution of DSI values was rather homogeneous, with values from most experiments centered around 70\% (Fig.5.7B). The half-time of recovery to control levels was $42.11 \pm 2.80 \mathrm{~s}$ (average from $\mathrm{n}=11$ experiments), which is not significantly different from the aforementioned average values for mIPSCs and sIPSCs $(\mathrm{p}>0.05)$.

Furthermore, it is extremely interesting to remark that there was no statistical difference between the DSI of the eIPSCs and of the sIPSCs ( $p>>0.10)$.

\subsubsection{DSI induction is not dependent on the intracellular solution of the postsynaptic cell}

Most experiments for this thesis were performed with a postsynaptic cesium-based solution; cesium works by blocking and/or reducing potassium conductances, thus leading to a strong increase in input resistance. This facilitated the clamping of the cell and the amplification of calcium entry into the cell by depolarizing protocols, but it clearly represented an unphysiological condition. This difference from "in vivo" conditions could be particularly important in Purkinje cells; indeed, these cells present a very high level of expression of functional potassium conductances, and, moreover, potassium A-like conductances have been suggested to play an important role in setting the amount of dendritic calcium increases in response to glutamatergic inputs onto Purkinje cells (Chan et al., 1989 and Midtgaard et al., 1993).

We, thus, decided to verify whether DSI was present in paired recordings also when the depolarization protocol was given to Purkinje cells recorded with a more physiological, potassium gluconate-based solution. The result I obtained was that, indeed, DSI could be seen also in these conditions, even though with a greater variability between pairs than with postsynaptic cesium. A typical DSI protocol is shown in Fig.5.8. Evoked currents in this intracellular solution were outward at the holding potential at which Purkinje cells were held. Over 11 repeated trials throughout the experimental period, the depression of eIPSCs due to DSI was on average $58.35 \pm 7.21 \%$.

Thus, also the calcium concentration increases due to depolarizations in potassium are sufficient to induce DSI, excluding possible artifacts coming from the use of cesium as main postsynaptic cation. 
A

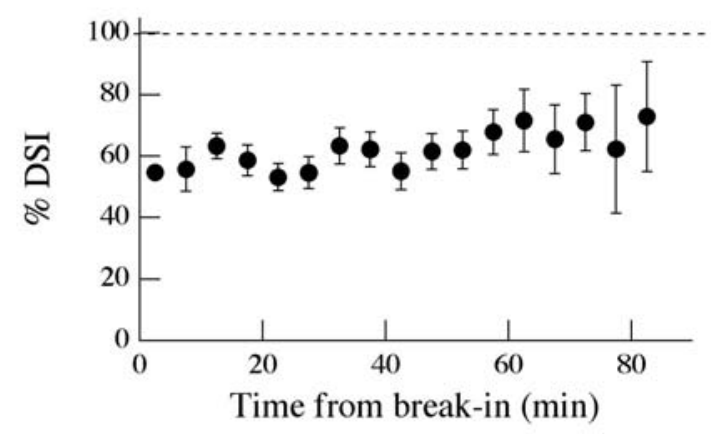

B

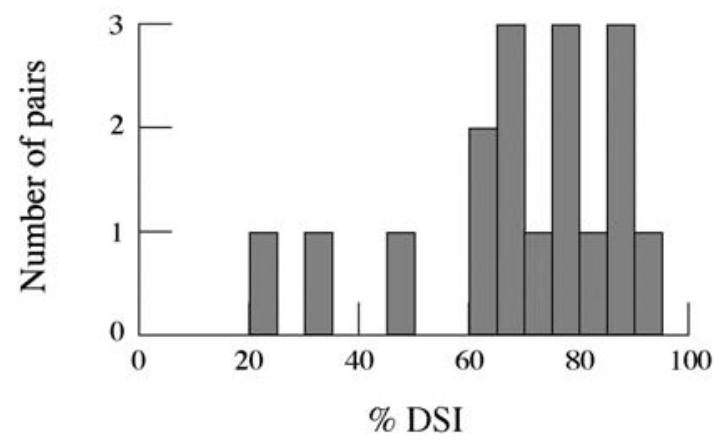

C

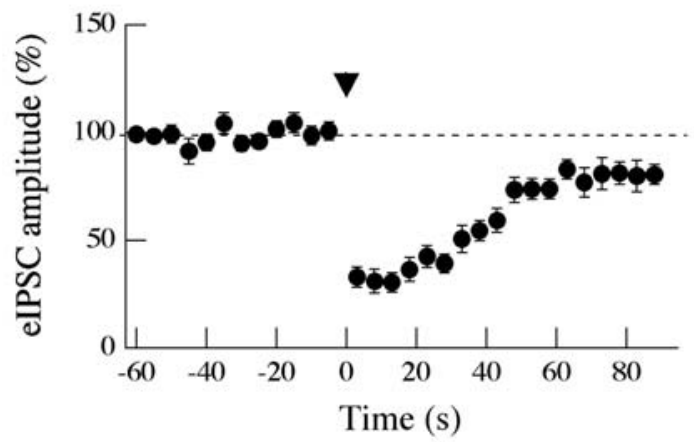

Figure 5.7: Time reliability of the induction and time course of the DSI of evoked IPSCs. 17 cell pairs have been pooled together to obtain the average values for this figure. In A, DSI values are plotted over time of postsynaptic whole-cell configuration, which was splitted into 5 min bins. Single DSI trials from each experiment were pooled according to their time of occurrence; thus, each value here represents average DSIs from several distinct experiments. It can be seen that DSI remains constant at least up to 90 min after breaking into the Purkinje cell. The first 8 eIPSCs during the test period were used. In B it is shown the distribution of DSI values for the same 17 paired recordings; notice that most DSI values are centered around $70 \%$. In $\mathrm{C}$, the normalized time courses for each experiment (itself calculated over several DSI trials) were averaged and again normalized over the $30 \mathrm{~s}$ preceding DSI. The time constant of recovery for the DSI of eIPSCs is not significantly different from the ones of mIPSCs and of sIPSCs, as explained in detail in the text. 


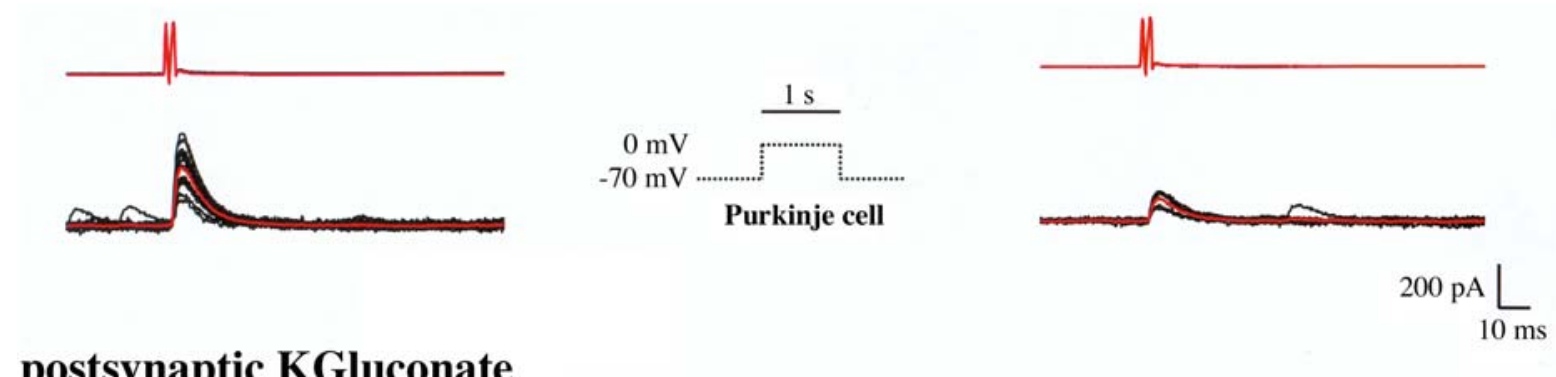

Figure 5.8: DSI induction was independent from the postsynaptic intracellular solution. A single DSI trial from a paired recording is shown, in which the postsynaptic intracellular solution was based on potassium as the main cation and gluconate as the main anion (this latter condition renders the GABAergic currents inward). This solution should in principle reduce the amount of calcium entry into Purkinje cells per depolarizing pulse with respect to a cesium-based solution and it should, thus, reproduce experimental conditions closer to a physiological state. The depolarization of the Purkinje cell induced DSI even with this experimental protocol; in this pair, DSI was $58.35 \pm 7.21 \%$ over $n=11$ repeated trials. Black traces in control (left) and DSI (right) are single evoked currents, red ones are their averages.

\subsubsection{Increase in the failure rate during DSI}

Previous experiment using extracellular stimulation suggested an increase in the failure rate during DSI (Vincent et al., 1992; Alger et al., 1996). However, any quantitative interpretation of these experiments was difficult because of uncertainties on the number of stimulated presynaptic cells and on the number and pattern of action potentials in individual interneurons per stimulation pulse.

An increase in the failure rate was readily apparent in virtually all DSI experiments performed in paired recordings (e.g. see Fig.5.9A for a typical experiment). To increase the number of sample data analyzed, for each DSI trial the failure rate during DSI was calculated for the first 8 eIPSCs following the Purkinje cell depolarization.

On average, GABAergic synapses onto Purkinje cells have been reported to have a high release probability in the juvenile (11 to 14 days old) rats, from which these data have been collected (Pouzat and Hestrin, 1997); my experiments confirm that, consistently with these results, the failure rate was very low in control conditions, amounting to $1.17 \pm 0.30 \%$ (ranging from $0 \%$ to maximum $3.19 \%$ ) of the evoked IPSCs ( $\mathrm{n}=15$ pairs). During DSI, the failure rate increased to $20.20 \pm 4.06 \%$ (range: $0 \%$, for 2 paired recordings where failures were not present in control 
A
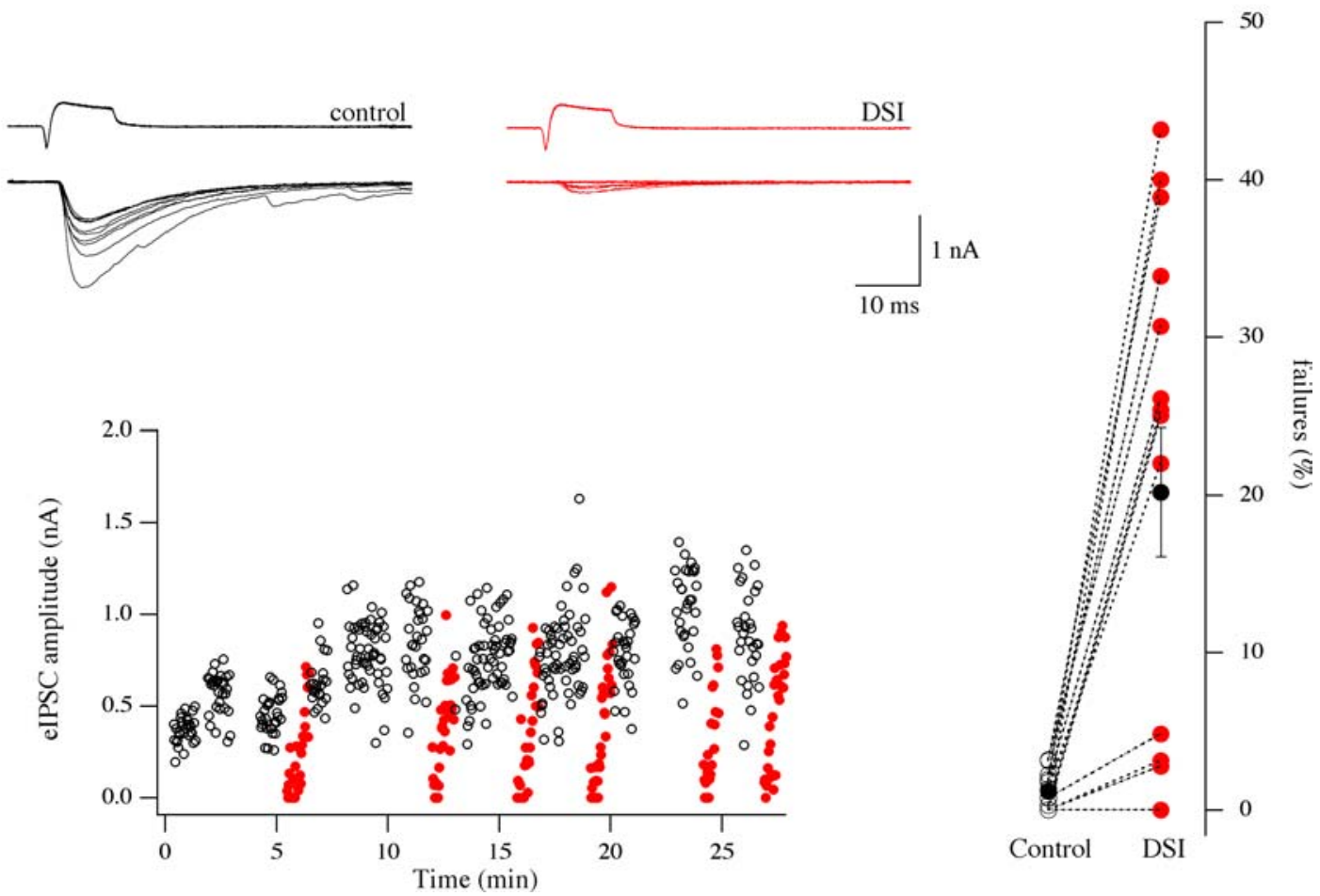

Figure 5.9: DSI was characterised by an increase in the rate of failures of evoked IPSCs. In A, a DSI trial from a paired recording shows the appearance of failures in transmission (right) in the test period, notwithstanding the remarkable strength of the synaptic connection in control (left traces). The time plot depicts an important element for this experiment, as well as for many paired recordings performed in order to characterise DSI: Rebound Potentiation (RP). The first depolarization protocol in the Purkinje cell induced DSI and, on a slower time course, a potentiation of transmission, which recovered to control levels (not shown in this example) in a range of times in the order of tens of minutes. As explained in the text, RP is a purely postsynaptic phenomenon: it changes neither the amount of DSI obtainable by the induction protocol nor the percentage of failures. In the experiment of part $\mathrm{A}$, the failure rate increased from $0.30 \%$ to $25.36 \%$ while DSI amounted to $73.11 \%$ ( $n=14$ DSI trials). In $B$, all the values from the 15 pairs where the failure rate was quantified are shown; in each experiment except 2 (where no failures were present either in control or in DSI) the failure rate increased during DSI. 
either, to $43.16 \%$; $\mathrm{p}<<0.05$, Wilcoxon matched-pairs rank test). Fig.5.8B shows the corresponding values for all the 15 pairs; in all, but the 2 aforementioned cell pairs where no change with DSI was detected, the failure rate increased significantly.

The lower graph of Fig.5.9A shows the time course for the eIPSCs for the experiment, from which the upper traces were extracted. It is worthwhile remarking the increase in the amplitude of the evoked IPSCs taking place after the first DSI trial in this paired recording. Usually recovering to control levels over several tens of minutes, this increase in synaptic transmission corresponded to Rebound Potentiation; this phenomenon has already been extensively described (Llano et al., 1991; Kano et al., 1992) and, as said in the introductory chapter devoted to DSI, RP is postsynaptically expressed, it requires higher calcium concentrations than DSI to be induced and, importantly, in my experiments it simply scaled up evoked currents without modifying the amount of inducible DSI.

\subsubsection{Paired Pulse Ratio (PPR) and DSI}

In the CA1 region of the hippocampus, there exist contradictory reports about the relationships between the Paired Pulse Ratio and DSI. One early paper claimed that DSI was not associated to any modification in the PPR (Alger., 1996). These data were subsequently challenged by another group, which reported an increase in the PPR during DSI, in line with a presynaptic site of expression for this phenomenon (Wilson and Nicoll, 2001).

In my experiments, presynaptic GABAergic interneurons were stimulated twice at $50 \mathrm{~Hz}$, once every $5 \mathrm{~s}$ (see some control and test evoked currents for one DSI trial in the left part of Fig.5.10Aa). For each DSI trial, the PPR was calculated as the ratio between the average amplitude of the second evoked currents and the average amplitude of the first eIPSCs for both control and test period. This avoids possible artifactual results in the evaluation of this parameter (Kim and Alger, 2001). As for the failure experiments, the first 8 eIPSCs during DSI were considered as test period: this was done to reduce the effect on the PPR of the great variability in the amplitude of the evoked currents at this synapse.

Interestingly, the first result of these experiments was that, in the control, these synapses are neither depressing (contrary to the previous reports, in both Pouzat and Hestrin, 1997 and Caillard et al., 2000) nor facilitating. The PPR for an interstimulation interval of $20 \mathrm{~ms}$ was $95.64 \pm 5.90 \%(n=11)$. As far as DSI was concerned, Fig.5.10B shows that in all the experiments $(n=11)$ the PPR increased in the test period (see the average left traces in Fig.5.10Aa). 
A

a

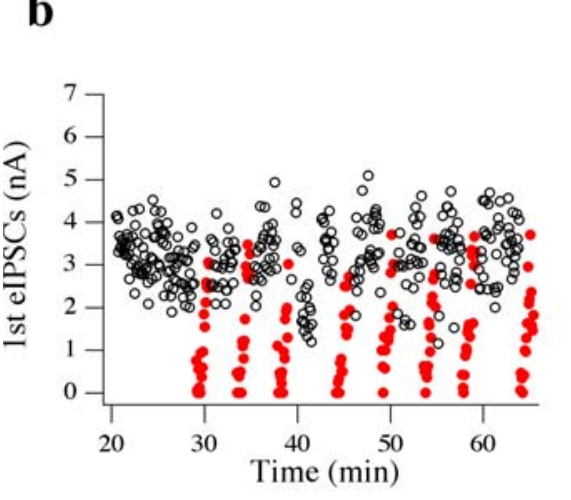

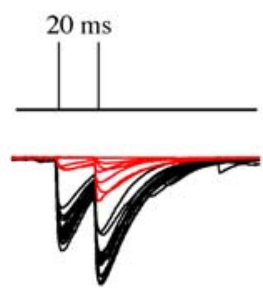

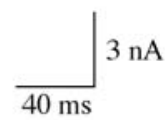
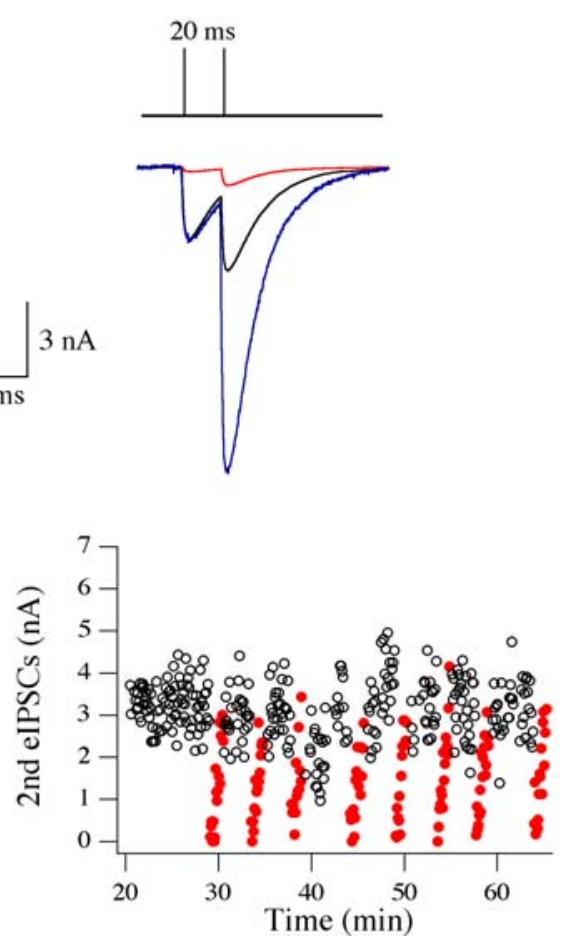

B

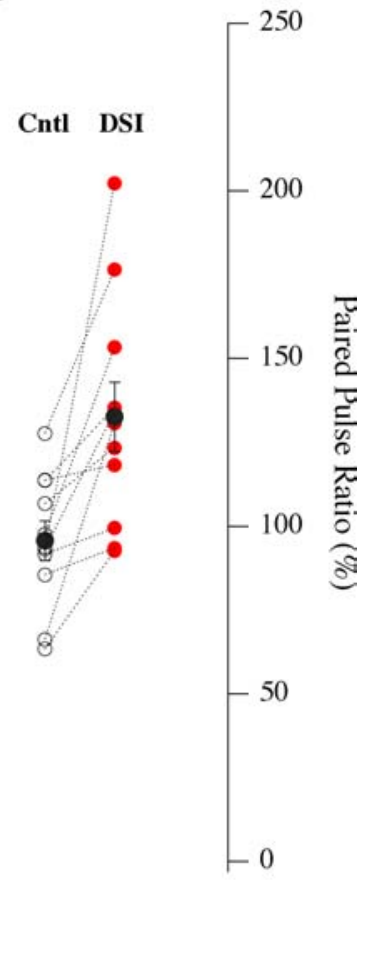

Figure 5.10: The Paired Pulse Ratio (PPR) increased during DSI. In paired recordings, presynaptic interneurons were stimulated twice at $50 \mathrm{~Hz}$ every $5 \mathrm{~s}$ and the ratio between the average amplitude of the second eIPSCs and of the first eIPSCs were quantified for the control and the test period. In the shown experiment, in a, control (black) and DSI traces (red) are shown on the left and their averages on the right. The blue trace represents the average currents during DSI scaled to the average amplitude of the first eIPSCs in control. Below, in b, the time plot further stresses the stability of synaptic connection throughout the whole experiment for both stimulations and the reliability of DSI induction. In this experiment, the PPR increased from $97.5 \%$ to $153.22 \%$ and DSI was $85.06 \%$ and $78.7 \%$ for the first and second eIPSCs, respectively $(n=8$ DSI trials). 
On average, the PPR value during DSI was $132.56 \pm 10.33 \%(\mathrm{p}<<0.05, \mathrm{n}=11$; Wilcoxon test) compared to the control value quoted above .

As expected, during DSI the second evoked IPSCs was significantly less inhibited than the first eIPSC: $47.11 \pm 4.84 \%$ compared with $58.62 \pm 3.83 \%(\mathrm{n}=11 ; \mathrm{p}<0.05$, Wilcoxon test $)$. Thus, DSI increased the PPR, consistently with a modulation of the presynaptic release probability.

\subsubsection{Kinetic parameters of eIPSCs and DSI}

Any change in the postsynaptic responsiveness to neurotransmitter and/or in the electrotonic properties of the postsynaptic membrane (and, thus, in the final somatic integration of dendritic synaptic currents) might be reflected in a change of the risetime and the decay time constant of the evoked currents.

I analyzed these parameters in paired recordings; the risetime was calculated as the time taken by the evoked currents to increase from $20 \%$ to $80 \%$ of their maximal amplitude. In control, the $20 \%$ to $80 \%$ risetime was $1.46 \pm 0.20 \mathrm{~ms}$ (range from $0.795 \mathrm{~ms}$ to $2.66 \mathrm{~ms}$ ) and the change in the test period was not significant to $1.57 \pm 0.22 \mathrm{~ms}(\mathrm{n}=13 ; \mathrm{p}>0.05$, Wilcoxon test $)$.

In all the experiments, a monoexponential fit well described the decay phase of the eIPSCs; at room temperature, where all these have been performed, the average decay time constant was $12.71 \pm 0.72 \mathrm{~ms}$ (range $6.36 \mathrm{~ms}$ to $15.55 \mathrm{~ms}$ ) in control conditions and $13.18 \pm 1.18 \mathrm{~ms}$ during DSI (range $8.58 \mathrm{~ms}$ to $19.85 \mathrm{~ms}, \mathrm{n}=10$; $\mathrm{p}>0.05$, Wilcoxon test), not statistically different. Thus, no change in these kinetic parameters was detected: this argues against a strong postsynaptic component of DSI and does not support the view that DSI simply scaled down eIPSCs without a direct action on the presynaptic release probability.

Another important parameter for evoked synaptic current is the latency between presynaptic action potential and postsynaptic response. Certain presynaptic mechanisms of inhibition, such as a reduced excitability of the axon arborization, could lead to a change in the latency of the evoked currents. The latency of eIPSCs was evaluated as the time between the peak of the presynaptic action potential, as recorded through the perforated patch, and the $20 \%$ point of the evoked current with respect to its maximal amplitude. No change was observed during DSI; latency went from $1.99 \pm 0.18 \mathrm{~ms}$ in control (range $1.39 \mathrm{~ms}$ to $2.85 \mathrm{~ms}$ ) to $2.04 \pm 0.17 \mathrm{~ms}$ during DSI (range from $1.15 \mathrm{~ms}$ to $2.85 \mathrm{~ms}, \mathrm{n}=11 ; \mathrm{p}>0.05$, Wilcoxon test).

See Fig.5.11 for the average and the detailed description of the changes of these parameters in each single experiment. 

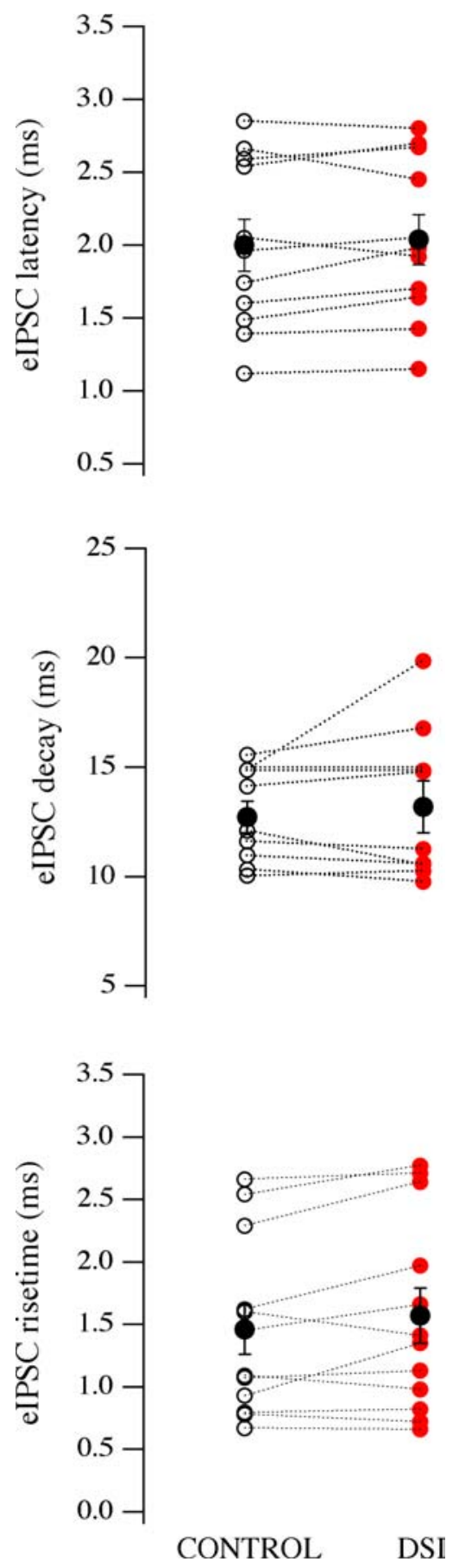

Figure 5.11: The kinetic parameters of eIPSCs were not modified during DSI. From top to bottom, the values for eIPSC latency ( $n=11$ experiments), decay time constant $(n=10)$ and risetime $(n=13)$ are shown for control (open circles) and test (filled circles) periods. Dotted lines connect the points corresponding to a same experiment. These three quantities were not affected on average by DSI, as shown by filled, black symbols, which are the average values from all the experiments. Decay time constant and risetime are parameters, which are typically analised to evaluate possible changes in postsynaptic electrotonic properties; their stability further supports the idea that postsynaptic modifications do not contribute in any form to DSI. Here, values during DSI have been calculated over the first 8 eIPSCs of the test period. 
For the same reasons as for the PPR measurements, the first post depolarization 8 eIPSCs were considered as test period for all the three properties.

Taken together, these results support the view that DSI is a purely presynaptic phenomenon.

\subsubsection{Coefficient of variation and DSI}

The so called Coefficient of Variation ( $\left.\mathrm{CV}=\frac{\text { standarddeviation }}{\text { mean }}\right)$ method has been extensively used in the past to study the locus of modification (pre versus postsynaptic) of synaptic transmission following induction of plasticity phenomena (see for example Bekkers and Stevens, 1990 and Malinow and Stevens, 1990 in the case of Long-Term Potentiation).

The "classical" study of variations in the CV is based on a simple binomial model representing quantal synaptic transmission; the invariance between release sites of the quantal parameters $\mathrm{n}$ (number of release sites), $\mathrm{p}$ (probability of release) and q (postsynaptic quantal size) is assumed. With this hypothesis, the amplitude of evoked synaptic currents and their variance $\sigma^{2}$ are respectively given by:

$$
\begin{gathered}
e I P S C=n \times p \times q \\
\sigma^{2}=n \times p \times q^{2} \times(1-p)
\end{gathered}
$$

In the case of a form of synaptic plasticity, the ratio between $C V_{\text {pre }}^{2}$ versus $C V_{\text {post }}^{2}$ becomes a function of the ratio between the eIPSC amplitudes after and before plasticity:

$$
\frac{C V_{\text {pre }}^{2}}{C V_{\text {post }}^{2}}=\frac{(1-p)_{\text {pre }}}{(1-p)_{\text {post }}} \frac{(n \times p)_{\text {post }}}{(n \times p)_{\text {pre }}}
$$

which becomes:

$$
\frac{C V_{\text {pre }}^{2}}{C V_{\text {post }}^{2}}=\frac{[(1-p) \times q]_{\text {pre }}}{[(1-p) \times q]_{\text {post }}} \frac{e I P S C_{\text {post }}}{e I P S C_{\text {pre }}} .
$$

Thus, for a form of synaptic depression like DSI (i.d.: $\frac{e I P S C_{p o s t}}{e I P S C_{p r e}}<1$ ), if $\frac{[(1-p) \times q]_{p r e}}{[(1-p) \times q]_{p o s t}}<1$, the reduction in synaptic strength is attributable uniquely to a decrease in $\mathrm{p}$.

The assumptions, upon which these equations are based, strongly limit the applicability and significance of this method: indeed, it is not straightforward that both release probability and quantal size are identical between release sites. This might be particularly true for synaptic connections presenting several, differently located presynaptic terminals; nevertheless, the CV method can be extended to more complicated cases, where, for example, a certain variability is 


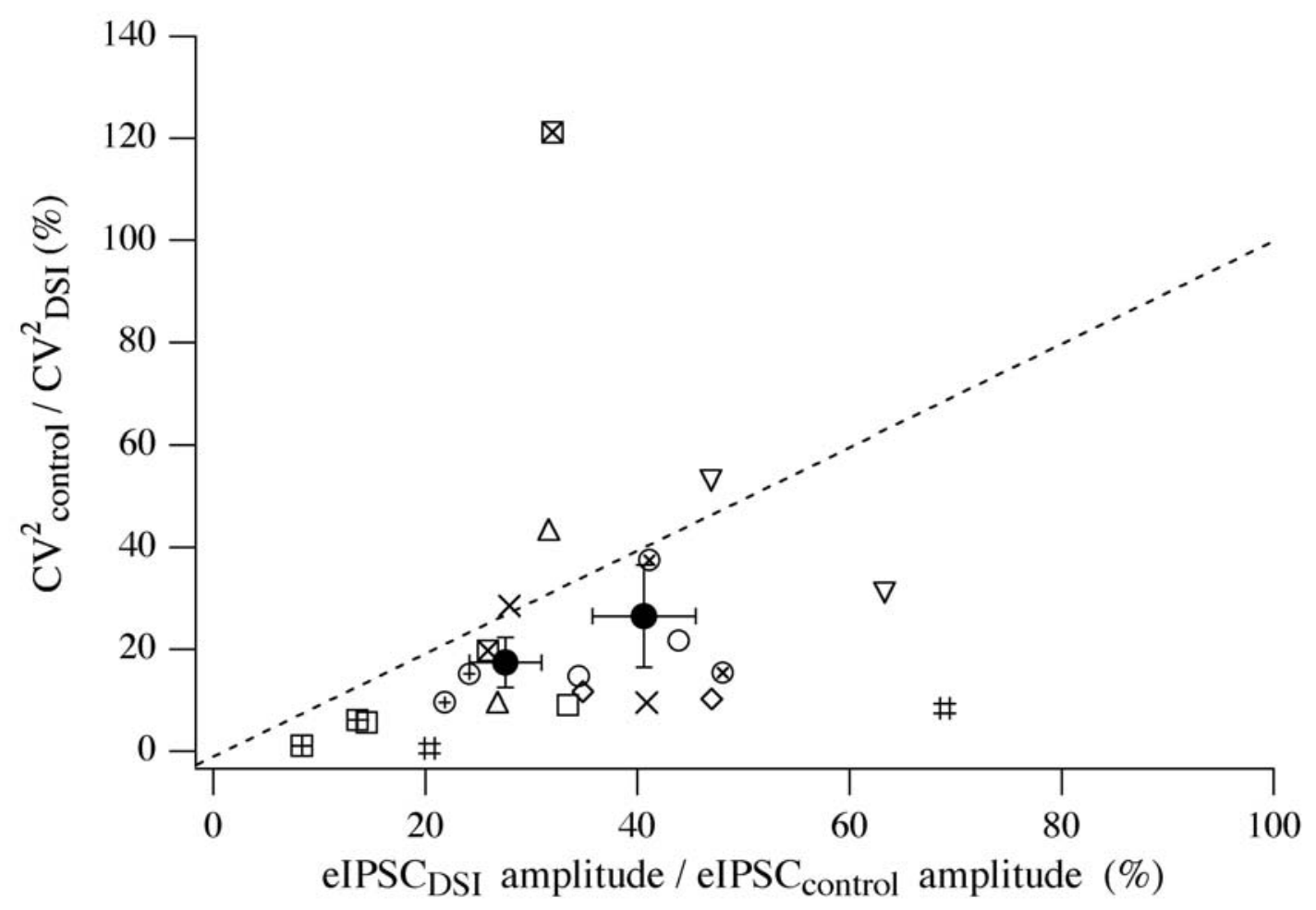

Figure 5.12: Analysis of Coefficient of Variation (CV) changes during DSI. As explained in more detail in the text, consecutive eIPSCs were divided into groups of 3 in order to calculate the synaptic parameters. During DSI, 2 triplets were created for each trial, covering the first 5 to 15 and the first 20 to 30 seconds following the induction protocol (presynaptic stimulation was at $0.2 \mathrm{~Hz}$ ); values from triplets were, then, normalized for the control. Each paired recording considered for this analysis $(n=11)$, so, contributed with two average values (depicted here by pairs of identical symbols), corresponding to these two successive test periods. Values for triplets from different experiments were then averaged (black filled circles). Simple, although limiting, hypotheses allow a binomial representation of synaptic transmission to produce information about the site of expression of plasticity phenomena. In the case of forms of synaptic depression, points below the diagonal are explained by a decrease in the probability of release $p$. The data coming from these experiments are consistent with this interpretation for DSI. 
allowed for all the parameters used (Faber and Korn, 1991). So, the behaviour of this parameter remains of great interest.

As seen, the recovery time of DSI was extremely fast in my recordings; it was, thus, fundamental to measure the $\mathrm{CV}$ of the eIPSCs over periods of (at least relative) stationarity of this phenomenon; looking at the time course of Fig.5.7C, we decided to divide the eIPSCs into groups of 3 consecutive events (triplets) for which to calculate the statistical parameters. Both the control and the test periods were partitioned in this way and, for each DSI trial, $C V^{2}$ and average amplitudes for the first two triplets during DSI were normalized for the corresponding averages in control. Finally, the values for all the distinct trials from an experiment were averaged; each paired recording, thus, gave a single contribution for each of the 2 triplets in DSI to the final pooled statistics.

The results from 11 paired recordings are illustrated in Fig.5.12; each couple of identical symbols pertain to the same experiment, the black filled circles represent the average values for the 2-post DSI triplets. As clearly shown, the great majority of the points, as well as the averages, are well below the diagonal line; this suggests a purely presynaptic site of expression for DSI, with a decrease in $\mathrm{p}$ mediating it. For the first and the second average triplet, $\frac{C V_{\text {pre }}^{2}}{C V_{\text {post }}^{2}}$ were 17.36 $\pm 4.9 \%$ and $26.47 \pm 10.07 \%$, respectively, with correspondingly amplitude ratioes of $27.54 \pm$ $3.42 \%$ and $40.62 \pm 4.89 \%(\mathrm{n}=11)$.

In our view, all the results shown in this chapter fully demonstrate the purely presynaptic nature of DSI; furthermore, these data suggest that a novel modulatory mechanism related to the generation and/or to the propagation of presynaptic spikes should be involved in DSI (see in section 7.1 how this consideration arises).

In the next chapter, this and other points concerning the nature of the retrograde messenger will be approached. 


\section{Chapter 6}

\section{Results: DSI and cannabinoids}

\subsection{Endocannabinoids are the retrograde messengers mediating DSI}

\subsubsection{Localization of CB1Rs in the cerebellum of juvenile rats}

Previous reports have shown a high level of expression of CB1Rs in the cerebellum (Tsou et al., 1998; Egertova and Elpick, 2000). Nonetheless, two main considerations have prompted us to reinvestigate the presence and precise localization of CB1Rs in our system. First, we worked with younger animals (11-15 days old rats) than in the previous studies (adult rats); synaptic structures and protein expression are known to differ between these two age groups. Secondly, although a strong CB1R expression level was detected in basket cell pinceaux and, more moderately, on basket cell terminal-like areas around Purkinje cell bodies, no clear demonstration was previously given of the presence of receptors in basket/stellate cell axons and synaptic terminals in the molecular layer.

The pattern of expression that has been obtained is illustrated in Fig.6.1. An antibody raised against the C-terminal portion of the protein was used. There is a strong, widespread punctate staining in the molecular layer, which shows the maximal expression of the receptor (Fig.6.1 A-D). This probably corresponds to parallel fibers running perpendicularly to the slice plane and is fully consistent with previous morphological (Tsou et al., 1998; Egertova and Elphick, 2000) and physiological (Levenes et al., 1998; Takahashi and Linden, 2000) data. No signal could be detected from Purkinje cell somata or dendrites (A-D) or in the smaller linear fibers belonging to Bergmann glial cells, which run centrifugally to the slice outer limit from the Purkinje cell layer (A, B). The signal abruptly ends at the border between molecular layer and 

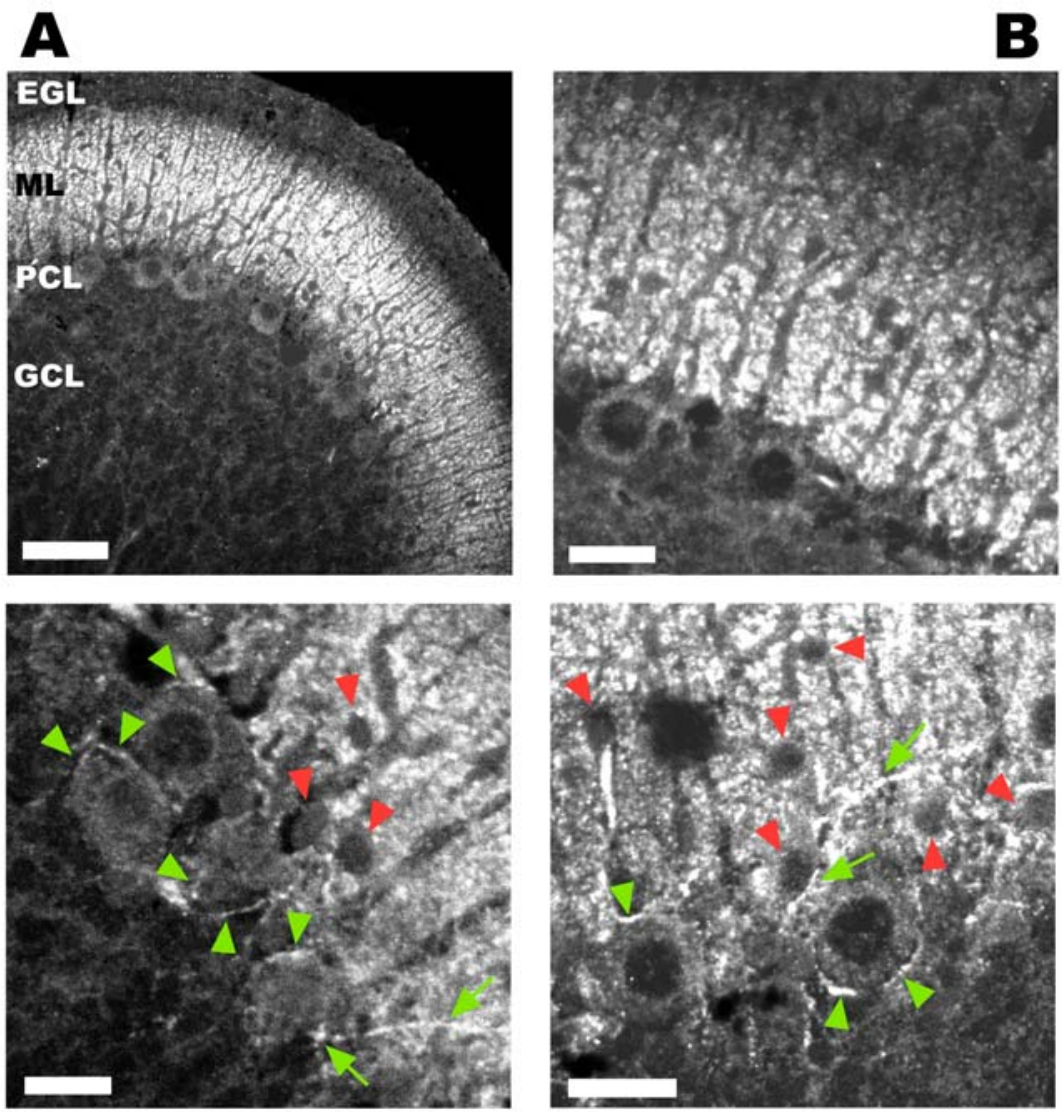

C

D

Figure 6.1: A-D: Immunohistochemical detection of CB1Rs in the cerebellum of a 12 days-old rat. A,B: Purkinje cell somata (large cell bodies with big dark nucleus in the PCL), dendrites and Bergmann glia fibers appear unstained. The strong punctate signal likely comes from parallel fibers. The EGL, which is still present at this developmental stage, is devoid of protein, probably due to the absence of parallel fibers and GABAergic fibers. A series of small, unstained somata can be seen in the lower part of the ML, presumably basket cell bodies. C,D: At high magnification near the PCL, punctate staining of the ML is clearly visible together with unstained cell somata (red arrowheads) corresponding to basket or stellate cells and/or migrating granule cells. Green arrowheads indicate the fluorescence surrounding Purkinje cell soma which is presumably due to basket cell synaptic terminals. Intensely stained basket or stellate cell axons contacting Purkinje cell somata are indicated by green arrows (C, D). Calibration bars: $50 \mu \mathrm{m}$ (A), and $20 \mu \mathrm{m}$ (B, C and D). Abbreviations in A: EGL=external germinal layer; ML=molecular layer; PCL=Purkinje cell layer; GCL=granule cell layer. 

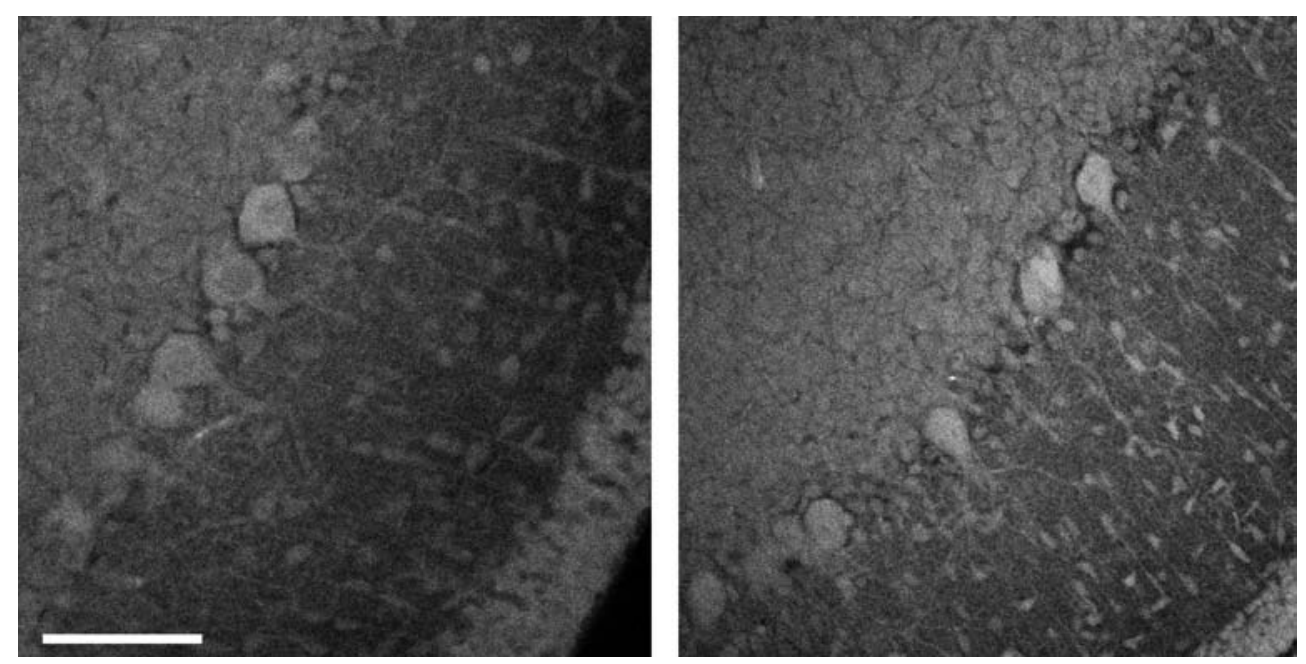

Figure 6.2: Two examples of confocal images from slices incubated only in the secondary antibody; notice that no aspecific signal can be detected. Calibration bar: $40 \mu \mathrm{m}$.

external germinal layer. Sometimes a faint mesh of fibers surrounding granule cell bodies could be detected in the granule cell layer. In B and, at higher magnification, in C and D (red arrowheads), cell bodies are not stained; more generally, no cell soma was revealed by the antibody in the molecular layer, suggesting that migrating granule cells, glial cells and, most importantly, GABAergic interneuron somata do not express CB1Rs. On the other hand, a strong signal was detected around Purkinje cell bodies in structures resembling basket cell synaptic terminals $(\mathrm{C}$, $\mathrm{D}$, green arrowheads).

Furthermore, and most importantly for our goals, fibers running longitudinally (C and D, green arrows) and giving out branches surrounding Purkinje cell somata were stained: these structures have all the morphological properties of GABAergic axons synapsing onto Purkinje cells. Sometimes, fibers running along Purkinje cell primary dendrites and extending in the granular layer were visible, likely corresponding to climbing fibers (data not shown). In slices incubated only with the secondary antibody, no aspecific signal was detected (Fig.6.2). These data indicate that, in young animals, CB1Rs are present in axons and synaptic terminals of cerebellar basket and stellate cells, as well as in parallel and climbing fibers.

\subsubsection{Implication of CB1Rs in DSI: paired recording experiments}

To study the possible involvement of cannabinoids in the DSI of evoked currents, we tested agents interfering with CB1R activity to investigate their effects on the DSI of evoked transmis- 
$\mathbf{A}$
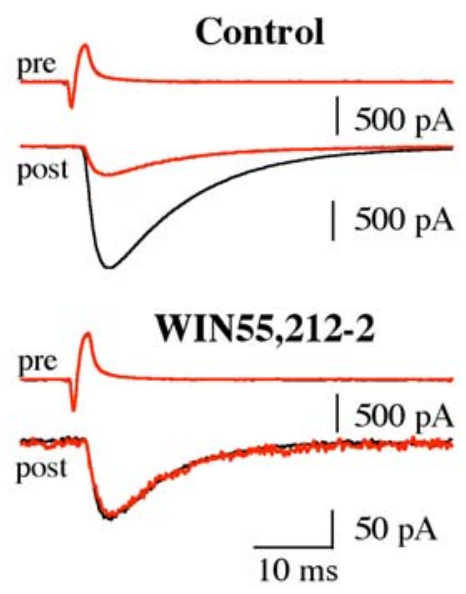

B

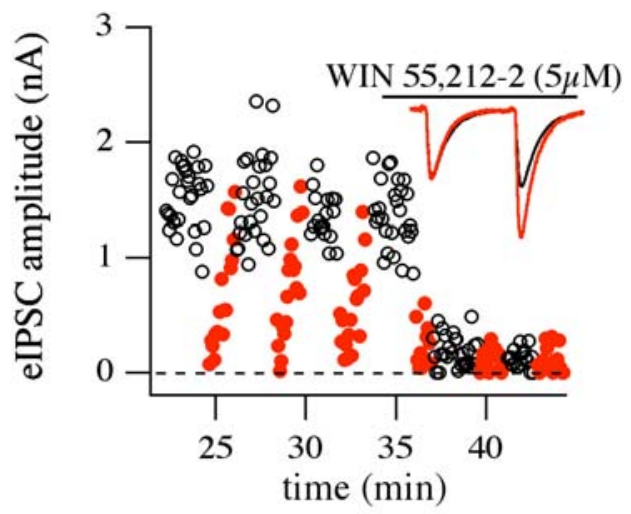

Figure 6.3: In paired recordings DSI is occluded by the CB1R agonist WIN 55, 212-2. A: Pre- and postsynaptic current sweeps averaged from one DSI trial, before (from 18 eIPSCs; black trace), and after (first 3 post-depolarization IPSCs; red trace) the depolarizing pulse. Upper panel: in control saline, the presynaptic current is not altered by the DSI-inducing protocol, while the postsynaptic current is strongly decreased (average control DSI: $86.9 \pm \mathbf{2 . 6 \%}, \mathrm{n}=6$ DSI trials). Lower panel: in the presence of $5 \mathrm{mM}$ WIN 55, 2122 , the postsynaptic current is markedly reduced (note 10-fold change in vertical scale). In addition, the red and black postsynaptic traces are now superimposed, indicating an abolition of DSI (average DSI in WIN 55, 212-2: $15.1 \pm 8.5 \%, n=4$ trials). B: Peak amplitude of individual eIPSCs plotted over time, same experiment as in $\mathrm{A}$. $\mathrm{t}=\mathbf{0}$ min corresponds to Purkinje cell break-in. Open circles correspond to control eIPSCs and red circles to eIPSCs measured 0-90 s after the DSI protocol. Inset: Averaged paired pulse currents elicited by 50 ms interpulse interval stimulations. Currents in WIN 55, 212, 2 (average of 55 traces; red trace) have been normalized with respect to the first paired evoked current in the control trace (average of 77 traces; black trace). In this experiment, the paired pulse ratio increases from $113 \%$ in the control to $182 \%$ in WIN 55, 212, 2, calculated as the ratio between the amplitude of the averaged second eIPSC and the amplitude of the averaged first eIPSC. 
A
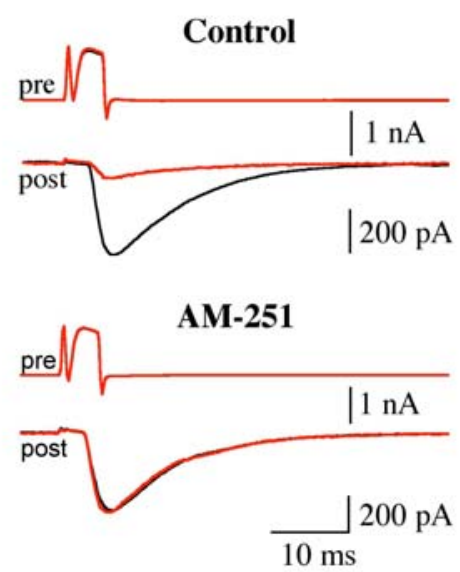

B

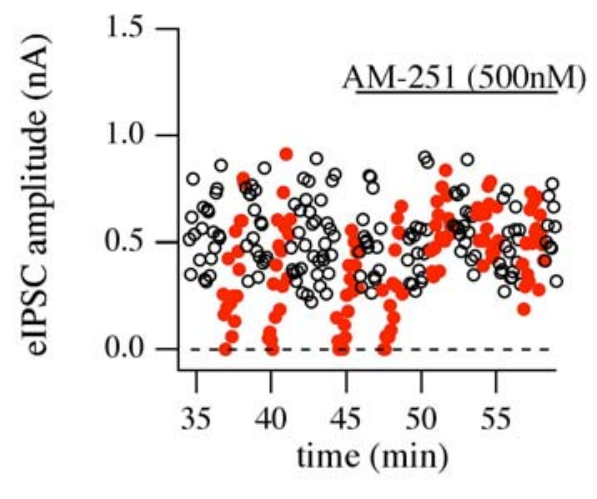

Figure 6.4: DSI is completely prevented by the CB1R antagonist AM-251. A: In this experiment, the initial amount of DSI observed in the control was $89.9 \pm 2.6 \%$ ( $\mathrm{n}=9$ DSI trials). Perfusion of $500 \mathrm{nM}$ AM-251 slightly increased the amplitude of eIPSCs, and markedly reduced DSI (to $15.2 \pm 5.0 \%$; $n=10$ trials, lower panel). No leak subtraction was applied to the presynaptic traces. B: Time plot of the effects of AM-251 for the same experiment. Same symbols as in Fig.6.3.

sion; experiments were performed in paired recordings between synaptically connected basket/stellate cells and Purkinje cells. DSI was assayed by depolarizing Purkinje cells to $0 \mathrm{mV}$ for $1 \mathrm{~s}$ at 3-4 minute intervals. eIPSCs were elicited every $5 \mathrm{~s}$.

Within minutes of perfusion with the cannabinoid agonist WIN 55, 212-2, the mean eIPSC amplitude was strongly reduced (to $10.3 \pm 2.1 \%$ of the control; $n=5$; Fig.6.3B). In the presence of WIN 55, 212-2, the paired pulse ratio at $50 \mathrm{~ms}$ interpulse interval was significantly increased (from $101.9 \pm 7.1 \%$ in the control to $143.4 \pm 10.8 \%$; $\mathrm{p}<0.05, \mathrm{n}=5$; see the traces in the inset of Fig.6.3B for one experiment), and the inverse of the square of the coefficient of variation $\left(\mathrm{CV}^{-2}\right)$ was very markedly decreased (from $21.5 \pm 9.3$ in the control to $0.96 \pm 0.25, \mathrm{p}<0.05$ ); both results suggest a presynaptic locus for the inhibition. In WIN 55, 212-2, the DSI protocol became ineffective (Fig.6.3A, left, lower panel). On average, the mean DSI value fell from 88.8 $\pm 3.7 \%$ in the control to $19.9 \pm 7.8 \%$ (Fig.6.5B, open bars). As shown in the summary plot of Fig.6.5A (open bars), the amplitudes of eIPSCs observed after a DSI protocol in control saline and before the DSI protocol in the presence of WIN 55, 212-2 were not significantly different. This suggests that the abolition of DSI seen in WIN 55, 212-2 is due to an occlusion effect and that WIN 55, 212-2 and DSI share a common pathway, though not necessarily implying that 


\section{A}
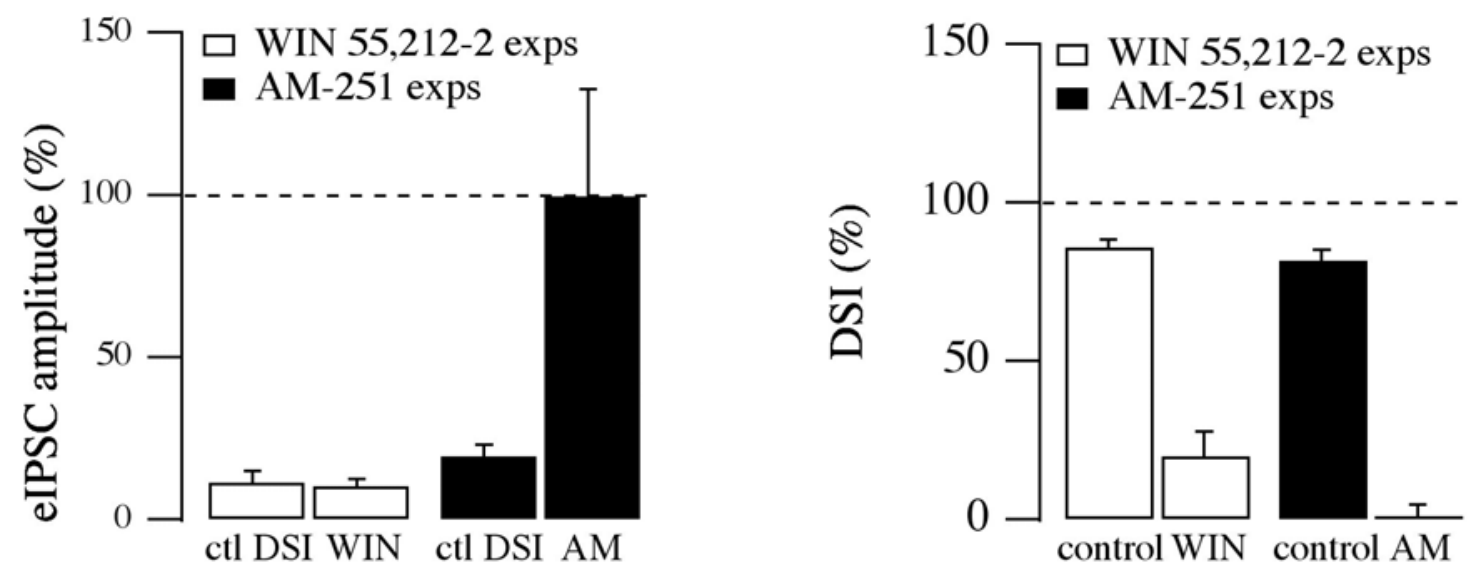

Figure 6.5: A: Histograms summarizing 5 experiments where the pharmacological test was with WIN 55, 212-2 (white bars) and 6 experiments with AM-251 (black bars); values are normalized relatively to the amplitude of eIPSC observed in control saline. ctl DSI bars are the mean eIPSC amplitudes during DSI in control saline. WIN and AM bars are the mean eIPSC amplitudes, respectively in the presence of WIN 55, 212-2 and AM-251. Note that WIN 55, 212-2 and the DSI protocol reduce the eIPSCs to about the same level, while AM-251 does not modify the amplitude of eIPSC. B: Same experiments as in A. Comparison of the amount of DSI observed in control conditions (control), and in the presence of either WIN 55, 212-2 (WIN) or AM-251 (AM). Notice the complete abolition of DSI in AM-251. 
DSI involves CB1R activation.

Fig.6.4 presents the results of the converse experiment, which consisted of exposing the slice to the CB1R antagonist AM-251. In control runs without DSI protocols, AM-251 had variable consequences on the amplitude of eIPSCs, but no net effect was apparent when averaging across experiments (mean ratio in AM-251 to control values, $99.5 \pm 33.0 \%$; $=5$, Fig.6.5A). AM-251 also failed to alter significantly the value of $\mathrm{CV}^{-2}$ (mean ratio in AM-251 to control values, $115.1 \pm 24.6 \% ; \mathrm{n}=5$ ). Thus, contrary to WIN 55, 212-2, AM-251 does not modify the basal synaptic transmission. However, AM-251 dramatically reduced DSI (control: $81.6 \pm 3.5 \%$; in AM-251: $1.1 \pm 3.6 \% ; n=5, p<0.05$; Fig.6.5B, filled bars), strongly suggesting that CB1Rs are involved in the establishment of DSI.

\subsubsection{Effect of CB1R activation and inhibition on mIPSCs}

We next asked whether CB1R agonists/antagonists would also act on mIPSCs. As reported earlier (Takahashi and Linden, 2000), WIN 55, 212-2 strongly inhibited the frequency of mIPSCs. This effect developed over a period of 10-15 min after addition of WIN 55, 212-2, at which point in time the reduction amounted to $55.6 \pm 6.7 \%$ of the control (Fig.6.6C; $p<0.05, n=10$ ), and it remained stable thereafter. At the same time period, WIN 55, 212-2 failed to affect the mIPSC mean amplitude (mean ratio to control: $90.0 \pm 10.7 \%, \mathrm{n}=10$; data not shown). These results suggest that activation of $\mathrm{CB} 1 \mathrm{Rs}$ leads to a decrease of the release probability of presynaptic vesicles. The effects of the CB1R antagonist AM-251 on mIPSCs were next investigated. On average, no significant effect on mean amplitude was observed. After 10-15 min in AM251, an increase in the mean frequency was observed (Fig. 3C; mean ratio to control values before AM-251 application: $1.33 \pm 0.06 \%$; $\mathrm{p}<0.05, \mathrm{n}=11$ ). However the significance of this effect was doubtful, because in many "sham experiments" without changing to AM-251, a spontaneous increase in frequency was observed, so that there was no statistical difference between AM-251 experiments and sham experiments. Thus the increase observed after AM-251 application could reflect a spontaneous upward trend of mIPSC frequency with time, rather than a genuine effect of AM-251. 

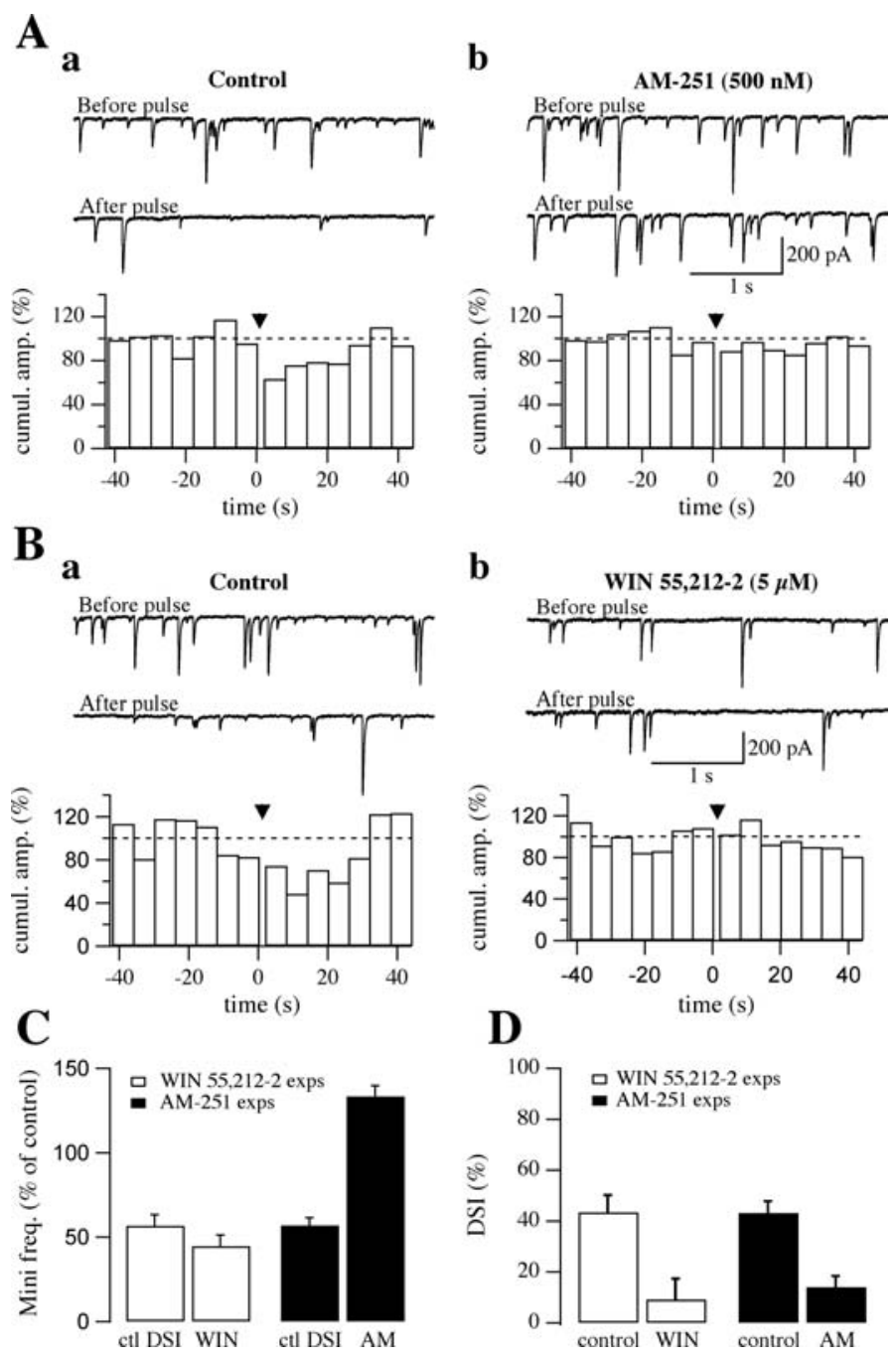

Figure 6.6: Effects of WIN 55, 212-2 and of AM-251 on DSI of mIPSCs. Here, DSI of mIPSCs was measured in TTX $(500 \mathrm{nM})$ following a 1s-long Purkinje cell depolarization. Sweeps are current samples recorded in Purkinje cells. A: Effects of AM-251. Aa: In control saline, the depolarizing pulse resulted in an inhibition of mIPSC frequency. Histogram: Averaged response to 4 DSI trials. DSI was measured as the percentage decrease in cumulative amplitude over a period of 2.5 to $12.5 \mathrm{~s}$ following the depolarizing pulse. Ab: In the presence of $500 \mathrm{nM}$ AM-251, DSI is markedly reduced (average histogram for 3 trials). B: Effects of WIN 55, 212-2. Ba: DSI in control saline. Bb: After perfusion with $5 \mathrm{mM}$ WIN 55, 212-2 mIPSC frequency was markedly reduced and DSI was abolished. C: Summary results from 4 experiments with WIN 55, 2122 (white bars) and 5 experiments with AM-251 (black bars). Ordinates indicate frequencies of mIPSCs (calculated over 5 min recordings) normalized with respect to the frequency of mIPSC observed in control saline before DSI. ctl DSI bars are the mean mIPSCs frequency during DSI in control saline. WIN and AM bars are the mean mIPSCs frequency, respectively in the presence of WIN 55, 212-2 and AM-251. D: Same experiments as in C. Comparison of the amount of DSI observed in control conditions (control), and in the presence of either WIN 55, 212-2 (WIN) or AM-251 (AM). Most of the experiments for this figure were performed by Carole Levenes. 


\subsubsection{Effect of CB1R activation and inhibition on DSI, as measured in miniature record- ing experiments}

Because DSI of mIPSCs (DSI ${ }_{m I P S C}$ ) and DSI of evoked IPSCs (DSI ${ }_{I I P S C}$ ) have different properties (reviewed in Marty and Llano, 1995), their sensitivity to cannabinoids may be different. Therefore, the effects of WIN 55, 212-2 and AM-251 were reexamined in the presence of TTX (500 nM), as illustrated in Fig.6.6. For these experiments, we used the same depolarizing pulse protocol as before (1s-long depolarizations to $0 \mathrm{mV}$, applied every 3-4 min). Fig.6.6A illustrates an experiment where AM-251 reduced DSI from 40.1\% down to 9.8\%, and Fig.6.6B shows another experiment with a DSI value of $31.8 \%$ in the control, which decreased in the presence of WIN 55, 212-2 to 9.8\%. Average results indicate that both compounds significantly reduce DSI $_{m I P S C}$ to about the same level (Fig.6.6D: AM-251 reduces DSI values from 43.1 $\pm 4.8 \%$ to $14.0 \pm 4.4 \%, \mathrm{n}=5$; WIN $55,212,2$ reduces DSI values from $43.4 \pm 6.8 \%$ to 9.1 $\pm 8.2 \%, \mathrm{n}=4 ; \mathrm{p}<0.05$ in both cases). These results show that the activation of CB1Rs is a necessary and sufficient condition for the DSI of both evoked and miniature transmission in the cerebellum. Nevertheless, in contrast to $\mathrm{DSI}_{E I P S C}, \mathrm{DSI}_{m I P S C}$ is still significant in AM-251 (p $<0.05)$. This residual DSI could be due to some glutamate released from depolarized Purkinje cell, as previously proposed (Glitsch et al., 1996). However, blockade of group II mGluRs did not further decrease the amount of DSI persisting in AM-251. Indeed, a residual $\mathrm{DSI}_{n I P S C}$ of $20.1 \pm 4.7 \%(n=4)$ was still obtained in the presence of a combination of the specific group II mGluR antagonist LY $341495(5 \mu \mathrm{M})$ and of AM-251.

\subsubsection{Effect of WIN 55, 212-2 on presynaptic calcium transients}

In an attempt to elucidate the mechanism of action of cannabinoids, we performed measurements of calcium transients in the axons and presynaptic terminals of interneurons, following procedures developed earlier in our laboratory (Forti et al., 2000). Interneurons were loaded from the soma with the calcium dye Oregon-Green1 (OG1). Axons were then imaged during stimulation of the cells with short trains of action potentials (4 spikes at $50 \mathrm{~Hz}$ ). This resulted in highly localized calcium transients in axonal "hotspots" that include en passant synapses and terminals contacting Purkinje cell somata (Forti et al., 2000). Rundown of calcium transients was corrected for by linear extrapolation. Upon application of WIN 55, 212-2 the kinetics of the transients remained unchanged, but their amplitude was decreased. Fig.6.7 illustrates one of these experiments, where the reduction was $34.2 \pm 4.6 \%$ after 15 min of application 
A
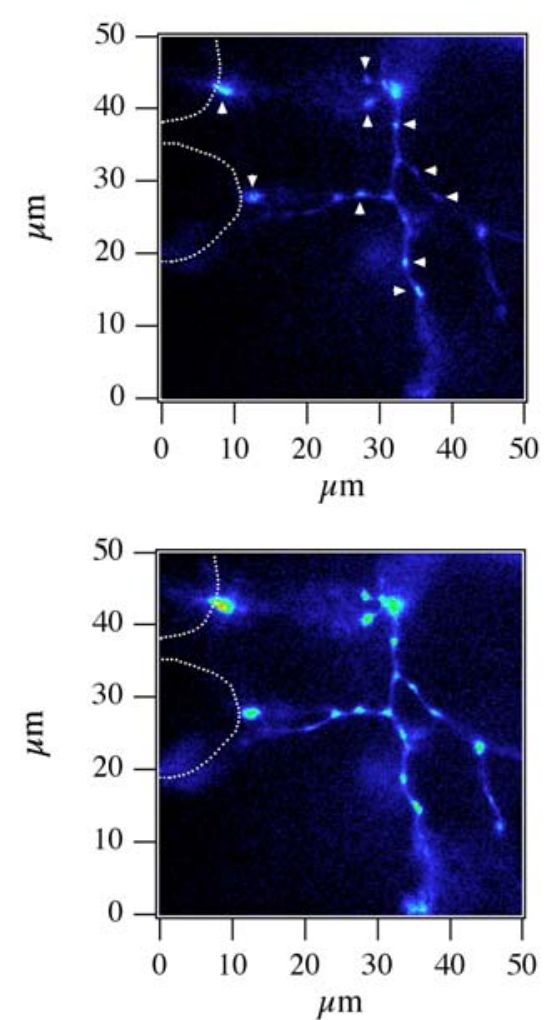

B
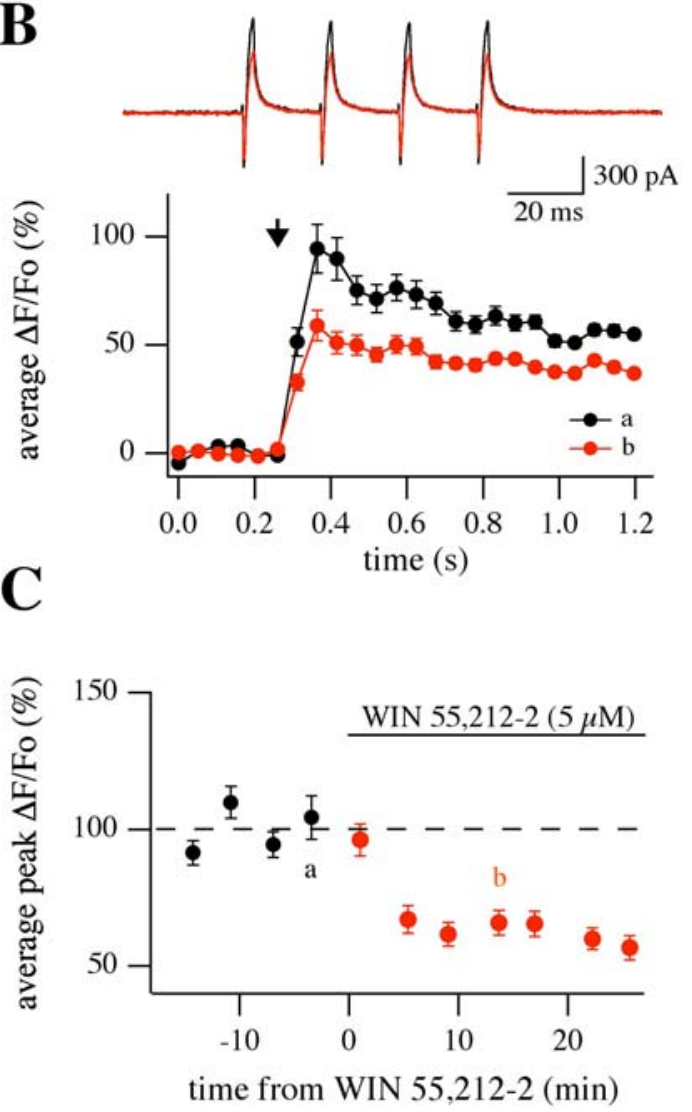

Figure 6.7: WIN 55, 212-2 inhibits presynaptic calcium transients A: $\mathrm{Ca}^{2+}$ transients observed in the axonal tree of an interneuron loaded with OG-1 $(250 \mu \mathrm{M})$. A: Peak response to a train of 4 action potentials in control saline. The upper panel shows the control level of fluorescence in the unstimulated axon, the lower one shows the increase in signal immediately after the depolarization train. White dots mark the position in the slice of two Purkinje cell somata contacted by axonal lateral branches of the recorded cell. The interneuron soma is about $20 \mu \mathrm{m}$ above, out of the imaged area. B: Analysis of $\mathrm{Ca}^{2+}$ transients for the same experiment as in A. Traces obtained in control and in the presence of $5 \mathrm{mM}$ WIN 55, 212-2 are labeled in black and red respectively. Upper panel: somatic action currents recorded during a train of 4 depolarizations. Lower panel: average $\Delta$ F/F0 signals for two different stimulations calculated from the 10 hotspots indicated by white arrowheads in A. The vertical black arrow indicates the time when the 4 spikes stimulus was applied. C: Time course of the effects of WIN 55, 212-2 for the same experiment. The points correspond to mean \pm s.e.m. of maximal $\Delta F / F_{0}$ changes after stimulation from the 10 hot spots. a and $b$ labels correspond to the data illustrated in $B$ in black and red, respectively. Values were normalized with respect to the four average control $\Delta \mathbf{F} / \mathbf{F}_{0}$. 
of the CB1R agonist. On average, the effects of WIN 55, 212-2 appeared as a reduction by $25.2 \pm 8.0 \%(\mathrm{n}=7$ experiments; $\mathrm{p}<0.05)$, which was complete 10 to 15 min after addition of the agonist. These experiments indicate that activation of CB1Rs leads to a reduction of action potential-dependent presynaptic calcium transients. In these experiments, we found no evidence of propagation failures in control conditions or after CB1R activation, confirming previous studies (Forti et al., 2000). However this does not rule out the possibility that DSI could involve enhanced propagation failures, as proposed in the hippocampus (Alger et al., 1996). Because we used 4 action potential stimuli, only massive failures would have been detected with our protocol. Furthermore, the experimental procedure for axon imaging involved dialysis of the intracellular milieu by the recording pipette, and this could have strongly influenced spike initiation and propagation properties.

\subsection{DSI, CB1R activation and interneuron firing frequency}

\subsubsection{Purkinje cell depolarizations inhibit firing in basket/stellate cells}

Does DSI affect the generation and/or the propagation of action potentials in molecular layer interneurons?

To answer this question, I made simultaneous recordings from stellate/basket cells and Purkinje cells. Both synaptically connected and not connected pairs were studied in this series of experiments. Interneurons were recorded in the current clamp mode of the perforated patch configuration, while Purkinje cells were voltage-clamped in the whole-cell configuration using a postsynaptic cesium gluconate-based solution with low concentration of chloride $(20 \mathrm{mM}$ $\mathrm{CsCl})$.

Small currents (normally in the order of very few pAmperes) were injected into interneurons to obtain, when necessary, a constant firing pattern. After 30/60 second-long control periods, Purkinje cells were depolarized for 1 second to $0 \mathrm{mV}$ to induce DSI. Fig.6.8 shows two DSI protocols from a single experiment; the interneuron in this case was synaptically connected with the Purkinje cell and the distance between the cell bodies was $22 \mu \mathrm{m}$; the upper traces are from the Purkinje cell, voltage clamped at $-65 \mathrm{mV}$, the lower ones represent the firing interneuron recorded in current clamp. The depolarization in the Purkinje cell induced DSI of the spontaneous IPSCs and, in the interneuron, a transient hyperpolarization: this led to an inhibition of the firing frequency, recovering to control levels in a few tens of seconds in parallel with the 


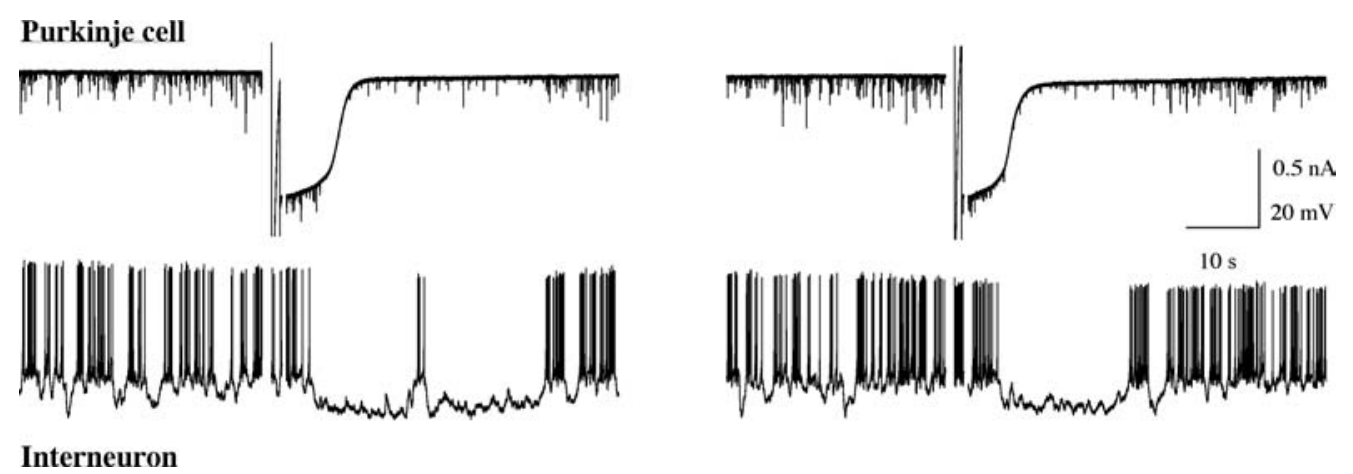

Figure 6.8: Two DSI protocols (left and right panels) are shown for a synaptically connected pair, given by a presynaptic low stellate cell (lower traces) and a postsynaptic Purkinje cell (upper traces); cell bodies were $22 \mu \mathrm{m}$ distant; the connection averaged $50.79 \pm 4.49 \mathrm{pA}(\mathrm{n}=43 \mathrm{eIPSCs}) .+0.5 \mathrm{pA}$ were injected in the current clamped interneuron to obtain a stable firing frequency; this cell fired in bursts. The depolarization in the PC inhibited the stellate cell firing by transiently hyperpolarizing it. See Fig.6.9 for the time course of DSI and firing inhibition in this experiment.

recovery of synaptic currents from DSI.

These two representive traces show a maximal inhibition of firing; in reality, there was a certain variability in the amount of decrease in firing for equal DSI-inducing protocols: for the same experiment as in Fig.6.8, Fig.6.9 shows the time course for the of DSI sIPSCs in Purkinje cells (left graph) and for the DSI of the spike frequency (right bar graph).

Studying this phenomenon in several cell pairs, it could be noticed that the inhibitory effect on synaptic transmission strongly depended on the distance between cell bodies. Fig.6.10 shows all the experiments performed with the same configuration as in Fig.6.8. Synaptically connected and not connected pairs were separately considered, to study whether the presence of synaptic terminals had an influence on the inhibition. A monoexponential fit of the amount of maximal inhibition as a function of distance for the two sets of data revealed no significant difference in the space constant for this phenomenon: $33.88 \mu \mathrm{m}$ and $31.23 \mu \mathrm{m}$ for connected and not connected pairs, respectively. On the contrary, a quantitative difference existed, once considering only pairs within a limited space distance; setting the threshold at $32 \mu \mathrm{m}$ (corresponding to 20 ticks on the scale of the microscope ocular), in connected pairs spike frequency was inhibited to $30.60 \pm 8.45 \%$ ( $n=13$ ) of control, while in unconnected pairs it was inhibited to a significantly different $51.85 \pm 6.76 \%$ ( $\mathrm{n}=9 ; \mathrm{p}<0.05$ ). Thus, inhibition at close range was $44 \%$ higher for structures which most likely presented closely juxtapposed membrane areas, as 

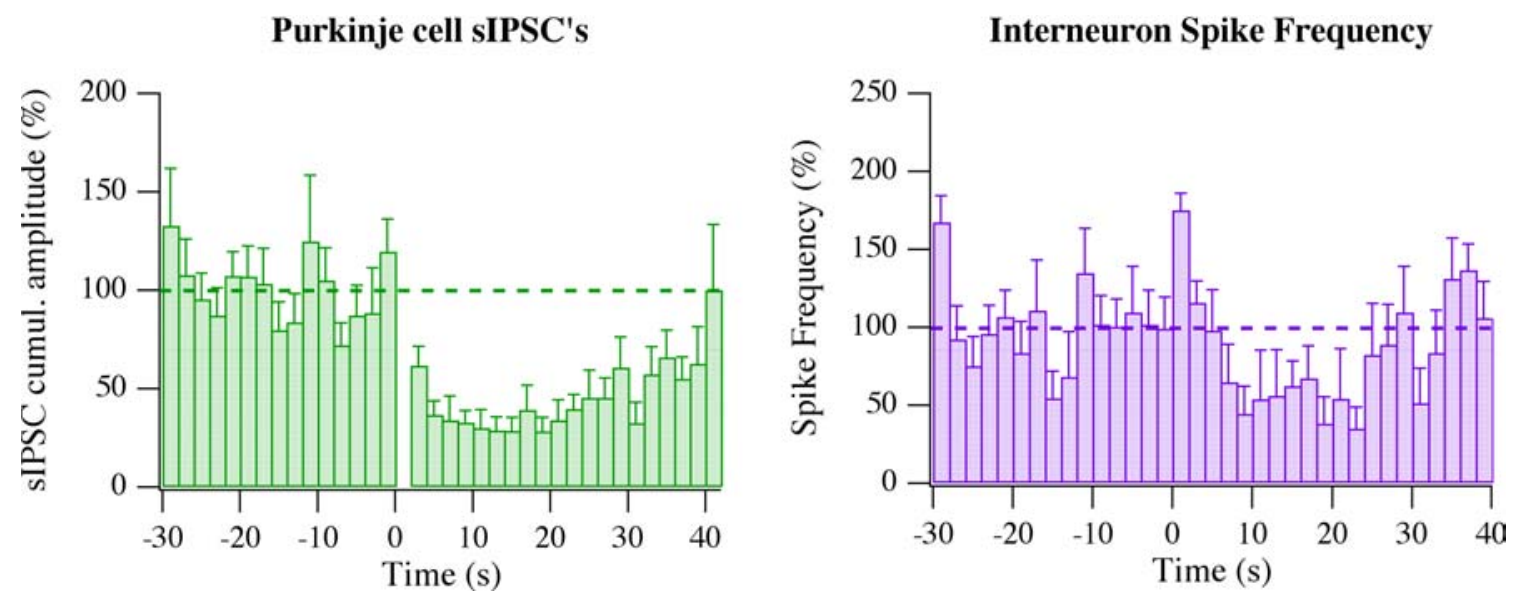

Figure 6.9: Time course for the DSI of the sIPSCs and of the firing frequency from the paired recording of Fig.6.8. Spike frequency went down to $54.35 \%$ with respect to the 30 s pre DSI control period, while the reduction of the sIPSC cumulative amplitude was $65.64 \%(n=7$ DSI trials). Values are calculated in the $4 \mathrm{~s}$ to $10 \mathrm{~s}$ period after the $\mathrm{PC}$ depolarization. The same binning, normalization and averaging protocols as for synaptic currents throughout this thesis were used for spike frequencies.

those mediating synaptic transmission are. Moreover, in the Purkinje cells from connected pairs DSI amounted to $75.75 \pm 3.54 \%(\mathrm{n}=13)$, while in unconnected PC DSI was $58.14 \pm 8.85 \%$ $(\mathrm{n}=9 ; 0.05<\mathrm{p}<0.10)$, thus not significantly different. Therefore, assuming a causal relationships between DSI induction and spike inhibition, the difference in firing inhibition cannot be attributed to a difference in the production of cannabinoids upon depolarization in the two groups of postsynaptic cells.

The maximal inhibitory effect of PC depolarizations in connected pairs reached even more dramatic levels when the spatial threshold was further decreased: considering only pairs within $25 \mu \mathrm{m}$ distance, interneurons fired at $9.78 \pm 3.47 \%(\mathrm{n}=5)$ of their control rate during DSI, while unconnected cells at $46.65 \pm 6.76 \%(\mathrm{n}=7 ; \mathrm{p}=0.005$ between these two groups). Purkinje cells were, thus, able to silence surrounding interneurons almost completely, provided that the distance was small enough.

Fig.6.11 shows the time course for spike inhibition for connected pairs (green-filled bars), unconnected pairs (violet bars) and for the cumulative amplitude of sIPSCs in Purkinje cells in control and after the $1 \mathrm{~s}$-long depolarizing step to $0 \mathrm{mV}$, which was given at $\mathrm{t}=0$. Shown data correspond to cell pairs at less than $32 \mu \mathrm{m}$ distance. First of all, it can be noticed that all along the DSI period, spike frequency was lower for connected pairs; moreover, the left y-axis has 


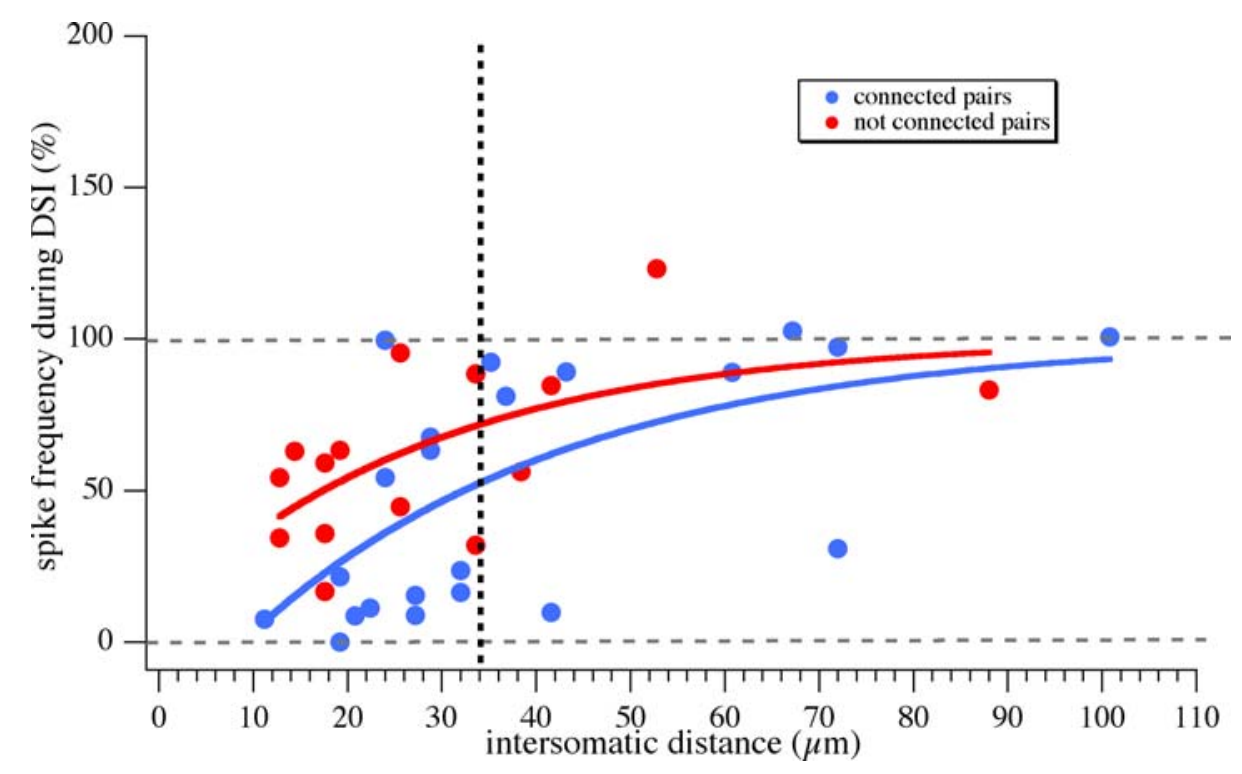

Figure 6.10: Dependence of firing inhibition with distance between interneuron and Purkinje cell cell bodies. Each point represents the interneuron spike frequency during maximal DSI plotted against intersomatic distance from one paired rrecording; blue points are for synaptically connected pairs $(n=22)$, red points for not connected ones $(n=15)$. The points for the two conditions were separately fitted with a monoexponential function constrained to a $100 \%$ at infinity. Space constants are $33.88 \mu \mathrm{m}$ and $31.23 \mu \mathrm{m}$ for connected and not connected pairs, respectively. 


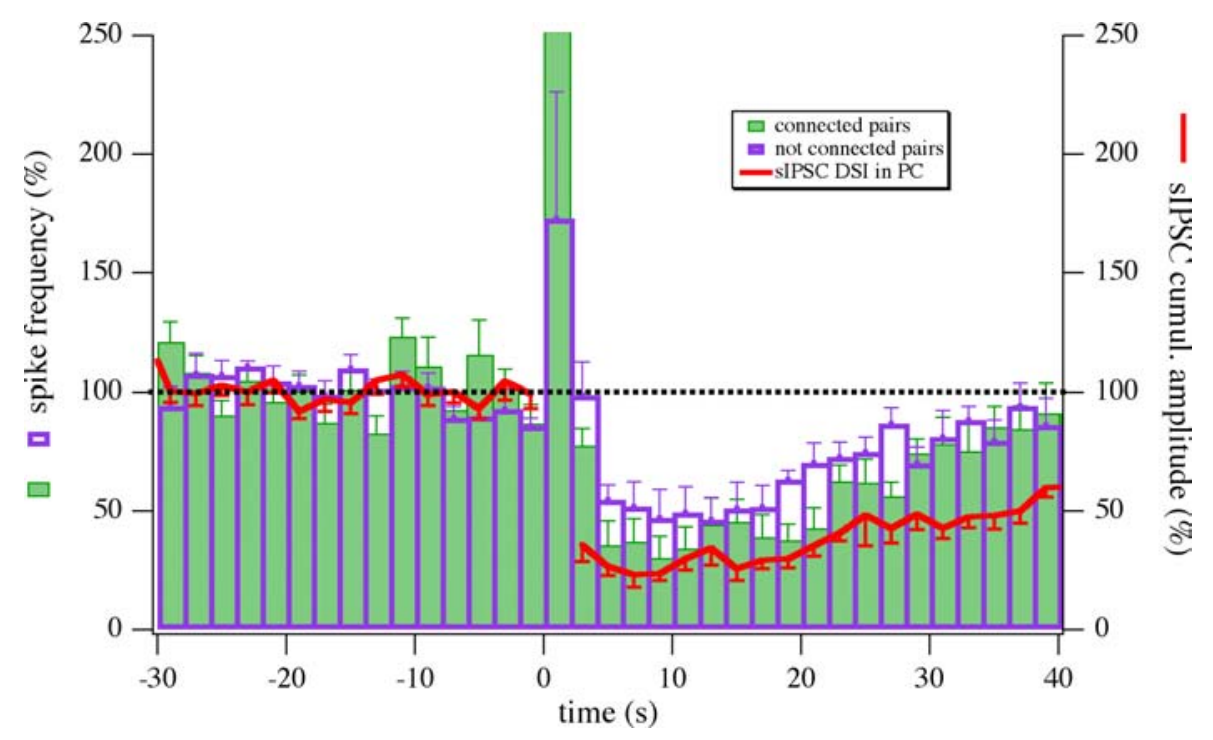

Figure 6.11: Time course for the DSI of the spike frequency in connected pairs (green filled bars), in not connected ones (purple bars) and for the DSI of the sIPSCs recorded in Purkinje cells. All values have been binned into $2 \mathrm{~s}$ periods, and values averaged for the control $30 \mathrm{~s}$ preceding DSI. A monoexponential fit with a constrained asymptotic value of $100 \%$ revealed that time constants for spike frequency were identical for connected and not connected pairs ( $\tau=\mathbf{2 6 . 3 2} \mathrm{s}$ ), while significantly longer for synaptic currents ( $\tau=\mathbf{5 8 . 8 2}$ s). Notice that the y-axis has been truncated for clarity: a clear facilitatory effect on firing is, nonetheless, visible shortly after $t=0$, when the DSI induction protocol starts.

been set to stress the time course of recovery of spike frequency from depression, nevertheless a big augmentation in firing frequency taking place at $t=0$ could be observed. I will return to this phenomenon in a later section.

The time courses for the DSI of sIPSCs and of spikes were different: monoexponential fits indicated that spike frequency returned to control level with an identical time constant $\tau=26.32 \mathrm{~s}$ for both connected and connected pairs, but with a slower time constant $\tau=58.82 \mathrm{~s}$ for spontaneous currents. This discrepancy suggests that spike frequency reduction cannot fully account for the DSI of spontaneous currents but that also another mechanism must be involved.

Finally, it is plausible to imagine that the stronger the synaptic connection, the more sites of contacts exist between presynaptic axon and postsynaptic Purkinje cell; in these conditions, the retrograde transfer of information between depolarized Purkinje cell and interneuron axons might be more effective in leading to a control of the firing frequency. This possibility was actually denied a relevant role in DSI by the data shown in Fig.6.12: in connected pairs at less than $32 \mu \mathrm{m}$ distance, evoked current amplitudes and spike frequency inhibition were infact not 


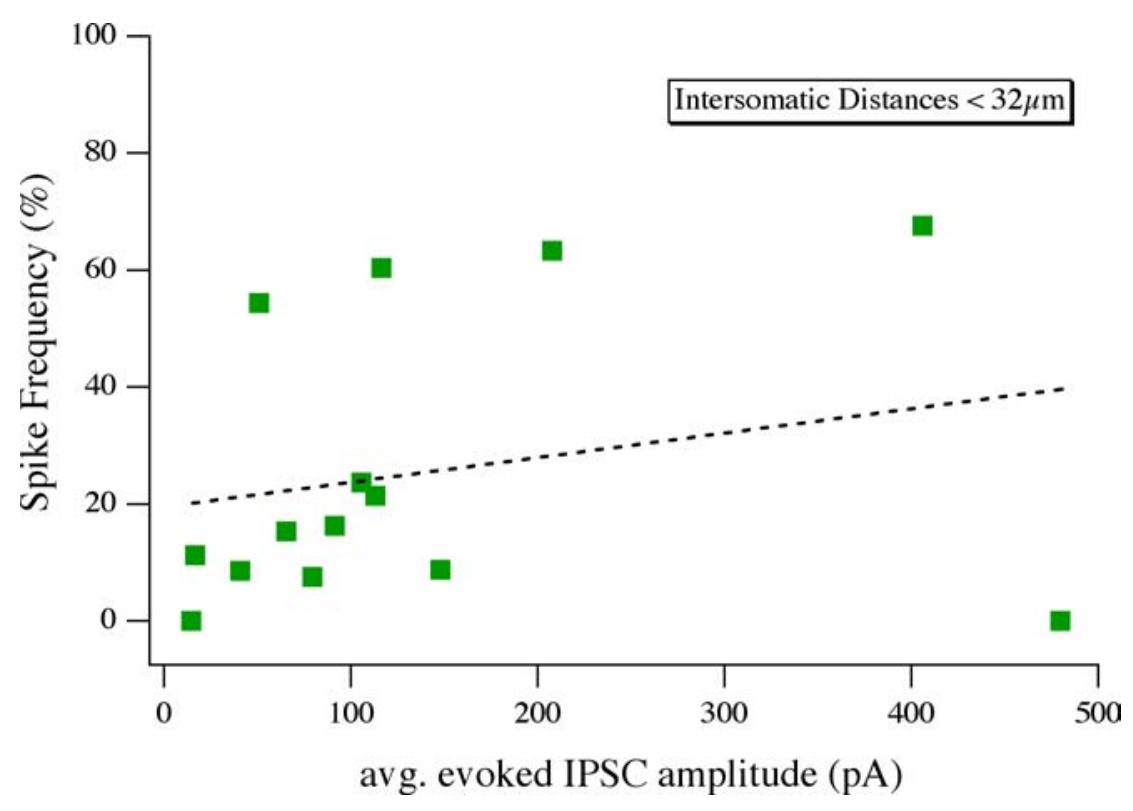

Figure 6.12: Plot representing spike frequency during DSI versus synaptic strength in connected pairs. Only distances less than $32 \mu \mathrm{m}$ have been considered $(n=13)$. The dashed regression line is a linear fit of the present data. The Spearman's correlation coefficient from these values is 0.38 and it indicates a not significant correlation $(0.1<\mathrm{p}<0.2)$.

significantly correlated.

\subsubsection{DSI-inducing protocols increase the threshold for action potential triggering in bas- ket/stellate cells}

A more direct assessment of the inhibitory effect of PC depolarizations on the firing of molecular layer interneurons is illustrated in Fig.6.13. Hyperpolarizing currents were injected to keep cells at around -60/-62 mV membrane potential (-5pA in the experiment shown); step depolarizing currents were then injected to make the cell fire at different rates and, thus, check for eventual changes during DSI. As shown, during DSI (blue traces both for the upper and lower series of traces, corresponding to the same DSI trial for this pair), the same amount of injected current (+5 pA upper, +10 pA lower) induced action potentials with a longer delay than in control; the delay increased for each consecutive spike during the $200 \mathrm{~ms}$-long depolarizing period. In some experiments (not in Fig.6.13), a small, transient hyperpolarization in the range of a few millivolts (generally from 1 to $3 \mathrm{mV}$ ) coincided with DSI. Altogether, the results are consistent 

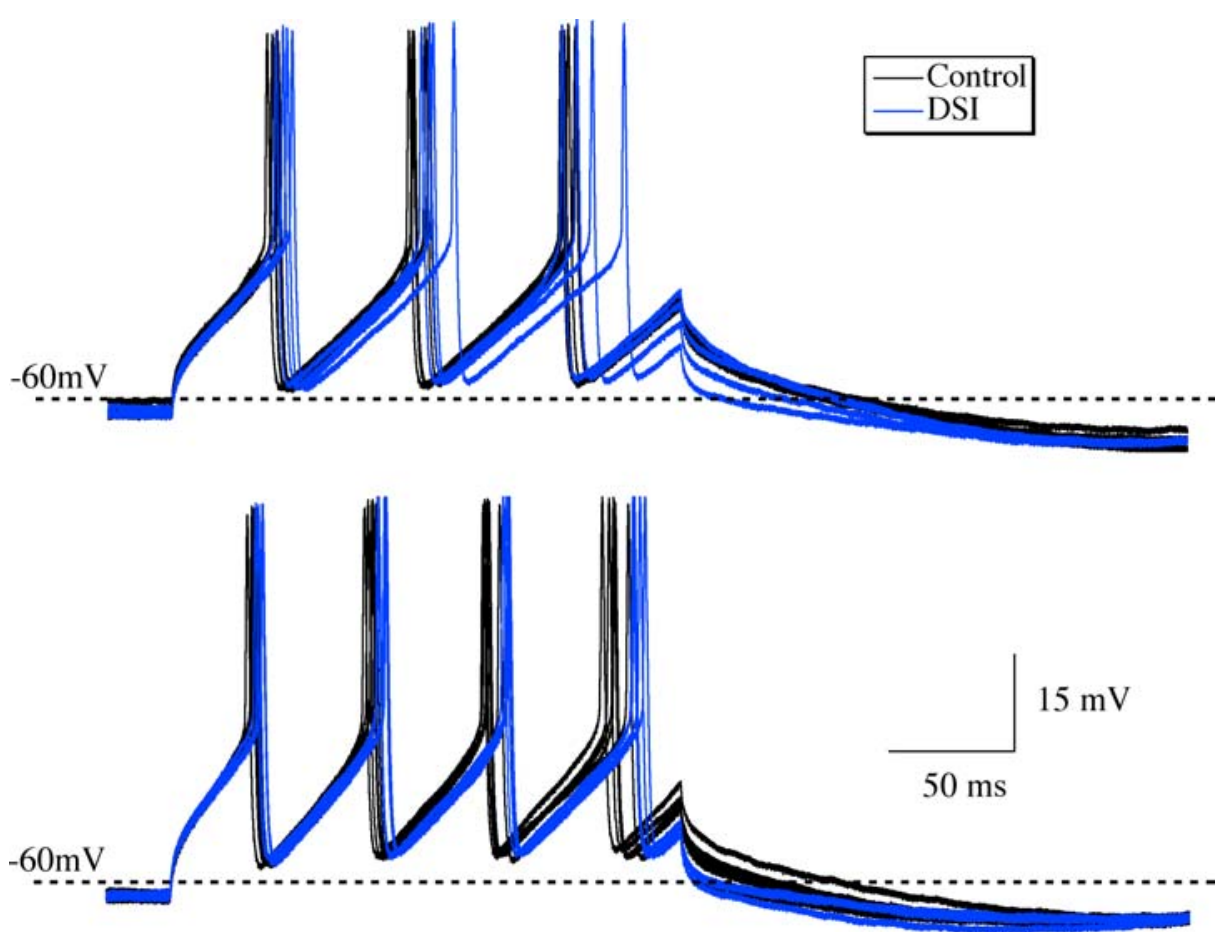

Figure 6.13: DSI is associated with an increase in the spike threshold of interneurons. These traces show 200 ms-long depolarizing current pulses ( $+5 \mathrm{pA}$ upper, $+15 \mathrm{pA}$ lower traces) given to a basket cell from a constant hyperpolarizing $-5 \mathrm{pA}$ current, to maintain it at around $-60 \mathrm{mV}$. In both upper and lower panels, 4 superimposed control (black traces) and 4 test current steps (blue traces) were given at $0.1 \mathrm{~Hz}$ before and after a DSI protocol in a nearby, synaptically connected Purkinje cell; the two cell bodies were $19.2 \mu \mathrm{m}$ distant. For both conditions, DSI is associated with a clear increase in the delay time of cell firing, which becomes more pronounced with increasing spike number. In some cases a small hyperpolarization of the cell membrane potential was detected (not visible in these examples). 
A
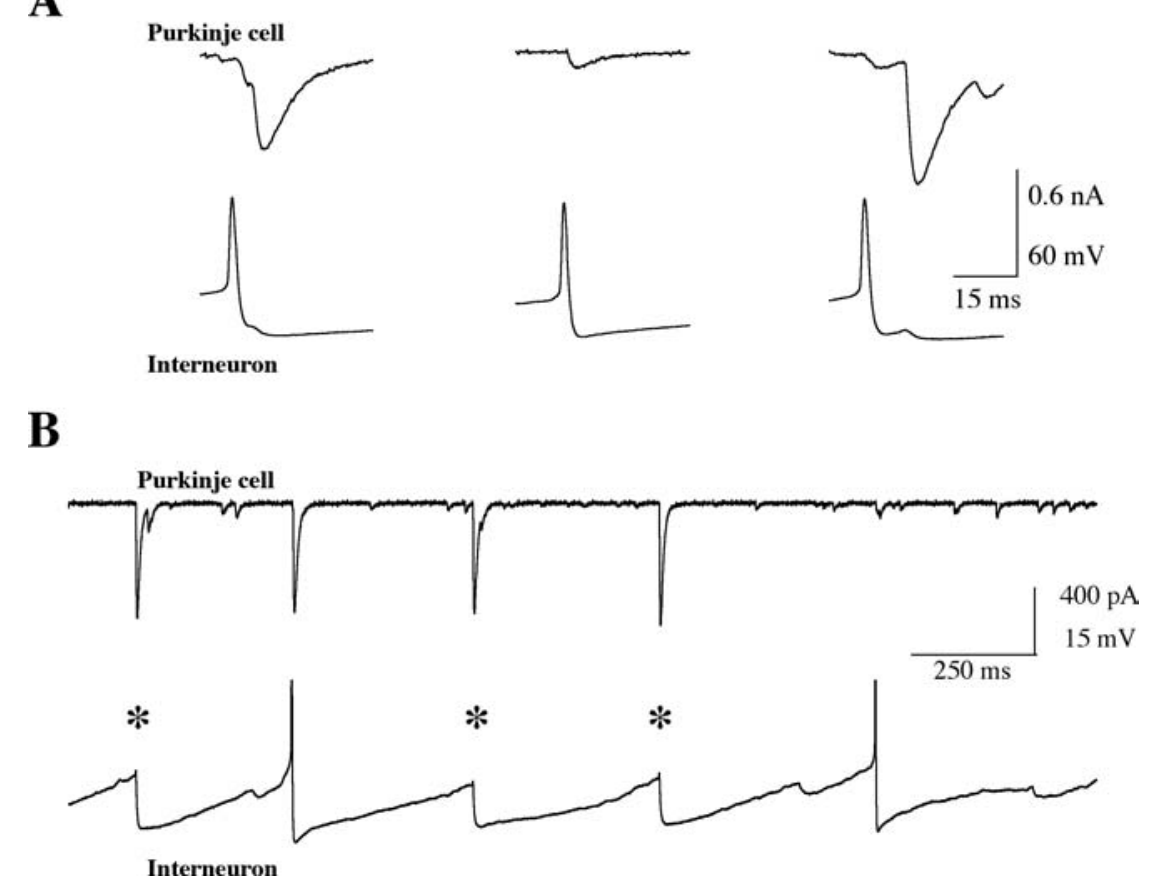

Figure 6.14: Traces from a current-clamped interneuron, synaptically connected to a postsynaptic Purkinje cell. In B, full action potentials in the recorded cell (truncated for clarity) are alternated to small depolarizing spikelets (black asterisks): spikelets and action potentials correspond to two different sets of spontaneous currents in the PC; as for the first full action potential from left in $\mathrm{B}$, in all the cases when a big IPSC appears to be associated with a full presynaptic spike, a careful look at the traces at faster time scales reveals that action potentials are followed by small spikelets and that, temporally, clearly distinct SIPSCs follow each other correspondingly in the PC (see for example first and third couple of traces in A). See text for average synaptic current values for each spike type.

with a change in the firing threshold of presynaptic interneurons and/or with a change in the resting conductance.

\subsubsection{DSI-induced inhibition of gap junctional spikelets in basket/stellate cells}

As mentioned in section 1.6, electrical synapses (i.d. gap junctions) permit the direct propagation of electrical signals between connected neurons through their characteristic low-resistance pathways.

Several predictions can be made about the properties of such signals, when recorded in electrophysiological experiments: for example, action potentials generated in a cell other than the 
recorded one will appear as depolarizing, small and fast deflections in current clamp and as downscaled and filtered action potentials in voltage clamp (we will call them in both cases spikelets). Furthermore, the electrical activity of nearby cells should be greatly affected by the transjunctional potentials deriving from the command voltages imposed on the recorded interneuron: this means, for example, that spikelet frequency will have to decrease with hyperpolarizations and increase with depolarizations. Finally, if actually representing action potentials generated in distant neurons, spikelets should be equally affected by Purkinje cell depolarizations as full action potentials are. We will see in these sections that all these predictions were indeed confirmed in my experiments. I will also show that paired recordings could provide a definitive proof that there actually exists direct electrical connections between our GABAergic interneurons.

So far, only two papers reported the electrical coupling via gap junctions of molecular layer interneurons in the cerebellum: Sotelo and Llinas, 1972 in adult cats and rats and Mann-Metzer and Yarom, 1999 in adult guinea pigs; no information was at present available about juvenile rats, where the importance of gap junctions (if not their same existence) was still controversial. Fig.6.14B illustrates a common finding in current clamp recordings from basket/stellate cells at depolarized membrane potentials: together with full spikes, small depolarizing peaks (spikelets: small black asterisks on the interneuron trace), often followed by more hyperpolarized potentials could be recorded. In the synaptically connected interneuron/Purkinje cell pair shown in Fig.6.14, small spikelets were associated with large sIPSCs in the Purkinje cells: the distribution of amplitudes of these synaptic currents was totally different from that of the evoked currents obtained by stimulating the presynaptic interneuron in voltage clamp (not shown). Fig.6.14A also shows that full presynaptic action potentials could be followed by spikelets (first and third couple of traces from right) and that, in the Purkinje cell, different synaptic currents were associated with these two distinct sets of presynaptic events; this was always the case when, at a slower time scale, big sIPSCs were apparently associated with full presynaptic spikes (as for the first action potential from left in the interneuron trace of Fig.6.14B). The average amplitude of this synaptic connection was, indeed, $65.56 \pm 1.95 \mathrm{pA}$ ( $\mathrm{n}=26$ eIPSCs) compared with the $698.36 \pm 25.73 \%(n=48)$ of the spikelet-locked events.

These small spikelets fulfilled many criteria identifying action potentials from electrically connected cells. Fig.6.15 shows that spikelets depended on the voltage of the recorded interneuron, suggesting an influence of the membrane voltage of one cell on the activity of a whole network 
A
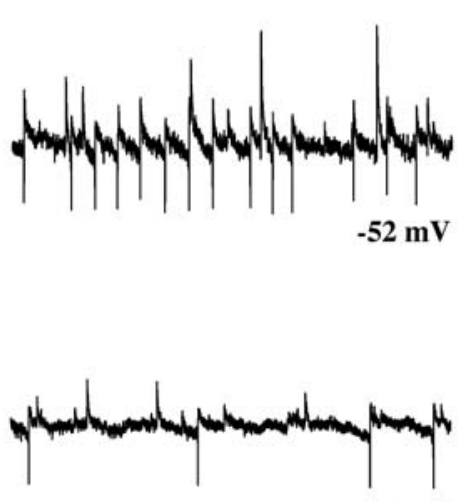

$-72 \mathrm{mV}$

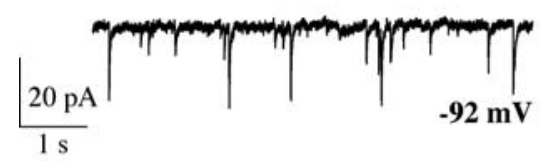

B
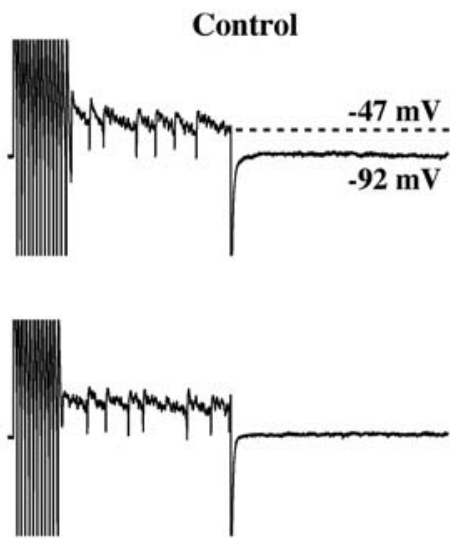

TTX $1 \mu \mathrm{M}$

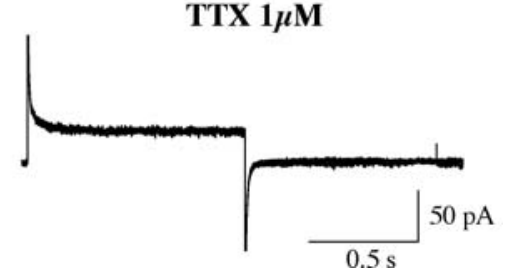

Figure 6.15: Spikelet voltage dependence: A shows that spikelet frequency critically depends on the recorded cell membrane potential. Notice that spikelets decreased in frequency as the membrane potentials was hyperpolarized. Only GABAergic sIPSCs could be detected at $\mathbf{- 9 2} \mathbf{~ m V}$, with opposite polarity with respect to those shown in the two upper traces. In $\mathrm{B}$, a different interneuron was voltage-clamped at $\mathbf{- 9 2 \mathrm { mV }}$ and $1 \mathrm{~s}$-long depolarizing pulses to $\mathbf{- 4 7} \mathrm{mV}$ were given; after a burst of unclamped action potentials (here truncated), spikelets appeared. These could not be detected at $\mathbf{- 9 2} \mathbf{~ m V}$, as shown in the 1 s-long post-step period. TTX in the bath $(1 \mu \mathrm{M})$ cancelled both types of events. In all these experiments, ionotropic GluR blockers were present in the bath. 
A

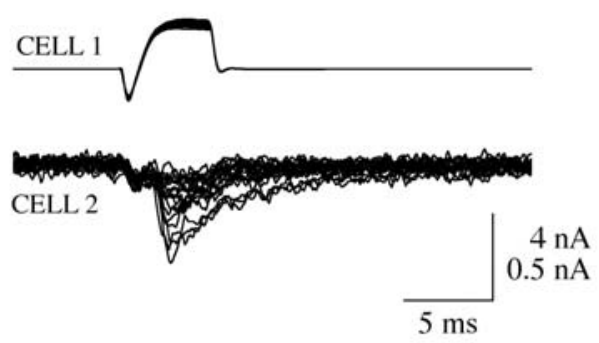

B
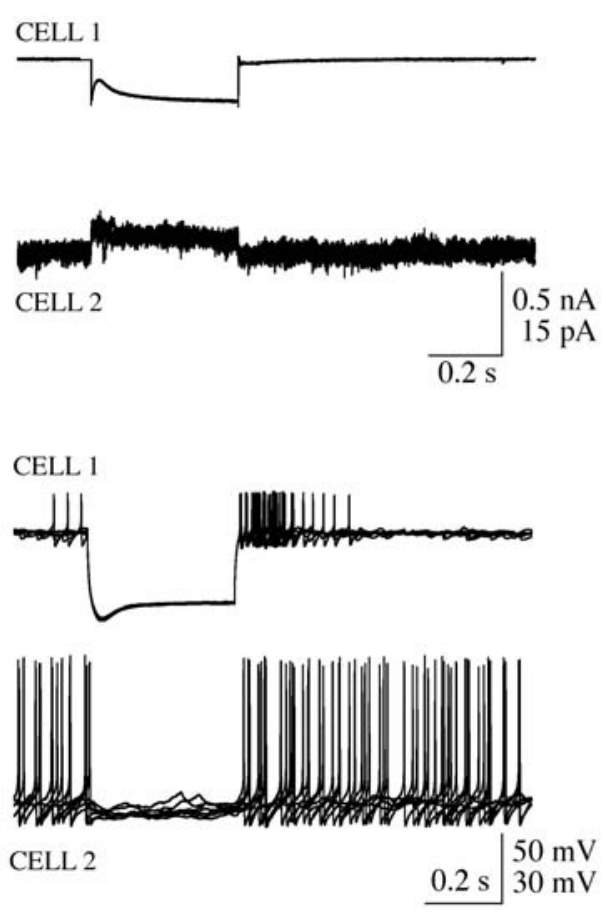

Figure 6.16: Mixed chemical/electrical signals in paired recordings between molecular interneurons; 2 low stellate cells at $37 \mu \mathrm{m}$ distance were simultaneously recorded in whole-cell with a potassium gluconate-based solution; in A, cell 1 was shortly $(5 \mathrm{~ms}$ ) depolarized from $-60 \mathrm{mV}$ to elicite an unclamped action potential: in cell 2 , small inward deflections were reliably induced in correspondance with the presynaptic action currents. These were followed by more variable and larger inward currents, with kinetics similar to GABergic synaptic events. Notice the difference in variability, time course and time of occurrence between the $\mathbf{2}$ sets of events. In trials not shown, an identical depolarizations of cell 2 induced only extremely reproducible inward currents in cell 1, with a small temporal delay. In B, cell 1 was hypepolarized for $500 \mathrm{~ms}$ from - $50 \mathrm{mV}$ to $-100 \mathrm{mV}$ : an inward current could be seen, with the classical slow onset of the hyperpolarization activated Ih conductance; in cell 2 at $\mathbf{- 5 0} \mathrm{mV}$, a squared outward current developed and returned to control levels in close time correspondance with the pulse in cell 1 . The same could be seen in cell 1 by hyperpolarizations of cell 2. Finally, in $\mathrm{C}$ both cells were current-clamped; hyperpolarizing pulses in cell 1 could fully inhibit tonic firing in cell 2; also the converse was true. 
of electrically, interconnected interneurons; in A, it can be noticed that the spikelet frequency diminished, the more the voltage-clamped interneuron was hyperpolarized; spikelets disappeared when the interneuron was kept at a very hyperpolarized potential $(-92 \mathrm{mV}$; notice also that GABAergic spontaneous currents were outward at $-52 \mathrm{mV}$ and $-72 \mathrm{mV}$, while changing polarity at $-92 \mathrm{mV}$ ); moreover, in $\mathrm{B}$ it can be seen that, in another voltage-clamped interneuron, 1 s-long voltage steps from $-92 \mathrm{mV}$ to $-47 \mathrm{mV}$ induced spikelets at the end of bursts of unclamped, full action potentials (upper two series of traces) and that TTX in the bath $(1 \mu \mathrm{M})$ canceled both sets of events (in $n=3$ interneurons, TTX was confirmed to fully inhibit spikelets). These spikelets did not depend on synaptic transmission: all the recordings were done with blockers of ionotropic glutamate transmission and the additional application of the GABAa receptor antagonist Bicuculline $(10 / 20 \mu \mathrm{M})$ had no effect ( $n=3$; data not shown). In more than $50 \%$ of my recordings, spikelets with these properties could be detected; moreover, in many cases, several spikelets of different shape and amplitude could be distinguished, suggesting the likely existence of widespread networks of several electrically and chemically interconnected GABAergic cells.

Importantly, direct electrical connections could be observed in simultaneous recordings from a pair of molecular layer interneurons. In Fig.6.16A, short depolarizations of one voltageclamped interneuron (cell1) from $-60 \mathrm{mV}$ to $10 \mathrm{mV}$ induced a mixed electrical/chemical response in the second cell (cell2). At first, a small inward current in the second interneuron (recorded at $-70 \mathrm{mV}$ ) was elicited in temporal correlation with the presynaptic unclamped action potential; short later, a larger inward current representing the evoked chemical IPSC could be observed. Conversely, the depolarization of cell 2 induced only an electrical signal in cell 1 (traces not shown). In B, prolonged hyperpolarizations of cell 1 from $-50 \mathrm{mV}$ to $-100 \mathrm{mV}$ induced an inward current in the same cell (with the characteristic slow onset of the inward Ih) and, correspondingly, a time locked outward current in cell 2 (at $-50 \mathrm{mV}$ ). Finally, C shows the superposition of several traces from the same 2 cells in current clamp: the injection of an hyperpolarizing current pulse in cell 1 totally inhibited firing in cell 2 . These behaviours were observed in several ( $n=4$ out of $n=7$ attempted pairs) paired recordings from molecular layer interneurons.

All these observations strongly suggest that interneurons form a network of extensively connected cells in the molecular layer, both through chemical synapses and electrical gap junctions. Importantly, Purkinje cell depolarizations induced the same pattern of early excitation followed 
A
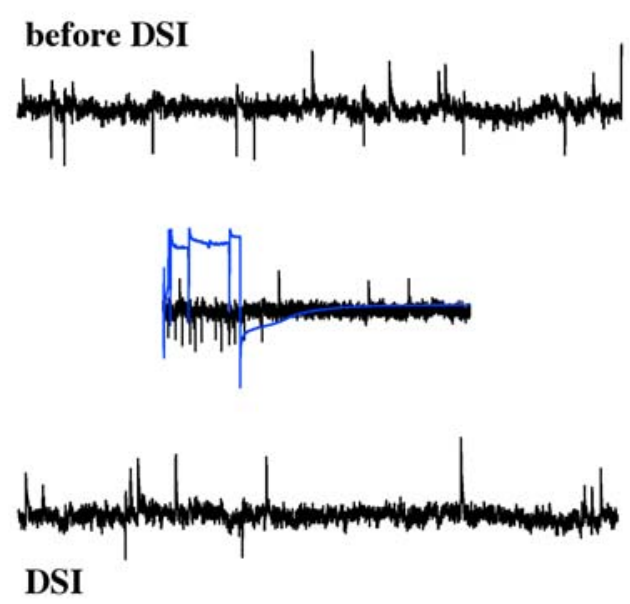

B
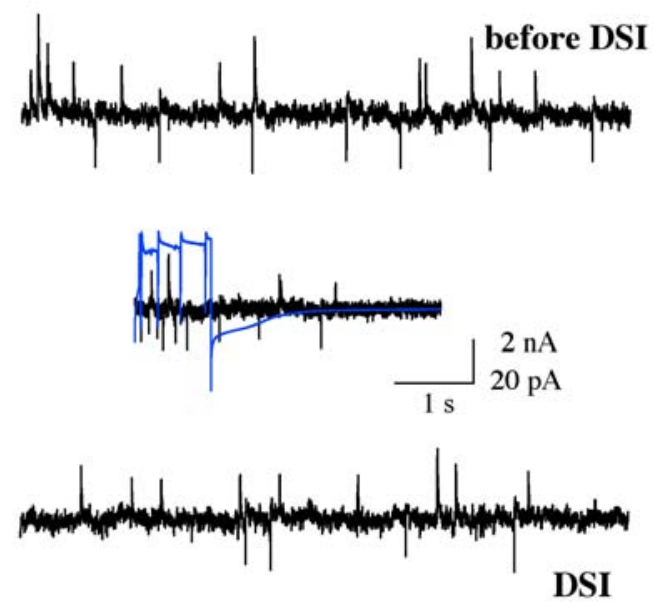

$20 \mathrm{pA}$

$1 \mathrm{~s}$

Figure 6.17: A and B show two DSI trials for a synaptically not connected basket/Purkinje cell pair, whose soma were $15 \mu \mathrm{m}$ distant. The interneuron was voltage-clamped in the whole-cell configuration at $\mathbf{- 4 7} \mathbf{m V}$ : in both A and B, upward (GABAergic SIPSCs) and downward (gap junctional spikelets) can be seen. Lower traces show that spikelets are inhibited in the first $\mathbf{5}$ seconds after PC depolarization withh respect to the control period (upper traces). The middle traces, where PC currents are shown in blue and interneuron currents in black, represent a 4 s-long period starting at the depolarization of the Purkinje cell; the acceleration of interneuron spike rate can be noticed. In this experiment, spikelet frequency decreased to $56.60 \pm \mathbf{5 . 7 8 \%}$ of control ( $n=4$ DSI trials), while during the PC depolarization it increased to $326.04 \pm 67.29 \%$. 
by inhibition, which was characteristic of full action potentials: in 5 interneuron / Purkinje cell pairs, in the depolarization period spikelet frequency increased to $563.89 \pm 203.12 \%$ of control (range from $176.72 \%$ to $1280.58 \%$ ), while, during DSI, it went down to $53.63 \pm 10.12 \%$ (range from $25.71 \%$ to $87.34 \%$ ). No information is, of course, available on the spatial location with respect to postsynaptic Purkinje cells of the interneurons from which these gap junctional spikelets may have originated; nevertheless, also this property strongly supports the hypothesis of a gap junctional origin for these events. Two DSI trials for one such experiment are shown in Fig.6.17. Notice the increase in spikelet frequency during PC depolarization (middle sweeps in both A and B: the blue traces represent the currents recorded in the Purkinje cell) and their inhibition in the period immediately following DSI (lower sweeps). See figure legends for more details. These results suggest that Purkinje cell depolarization can exert a modulatory role on the firing frequency of several interneurons of the molecular layer. Electrically and chemically connected GABAergic interneurons can form widespread (up to the millimeter range) networks of synchronously active cells (Tamas et al., 2000; Traub et al., 2001); the phenomenon described in this paragraph could, thus, endow single Purkinje cells with the possibility of indirectly controlling the coordinate activity of a vast population of cells and, thus, to regulate the output of the cerebellum over extremely wide regions.

\subsubsection{The inhibition of interneuron firing is mediated by endogenous cannabinoids}

Is the inhibition of action potential firing in interneurons induced by the same mechanisms as the DSI of synaptic currents?

To test this, I have studied the possible involvement of endogenous cannabinoids using paired recordings between interneurons and Purkinje cells. After verifying a strong inhibitory action on presynaptic firing by PC depolarizations in control DSI trials, in $n=5$ experiments I applied the specific CB1R antagonist AM-251 $(1 \mu \mathrm{M})$, before a new series of DSI test trials. One such experiment is shown in Fig.6.18 for a synaptically connected pair, in which the cell bodies were $27 \mu \mathrm{m}$ distant. As shown in A, the depolarization of the Purkinje cell leads to a strong DSI of sIPSCs in the same cell (upper traces) and, contemporaneously, to the silencing of the presynaptic stellate cell recorded in current clamp through the perforated patch (lower traces); in $\mathrm{B}$, the application of AM-251 is shown to greatly diminish both effects, consistently with the previously reported effect of CB1R block on the DSI of synaptic currents and, furthermore, also suggesting that endogenous cannabinoids modulate the spike rate of interneurons. $\mathrm{C}$ and $\mathrm{D}$ 
A
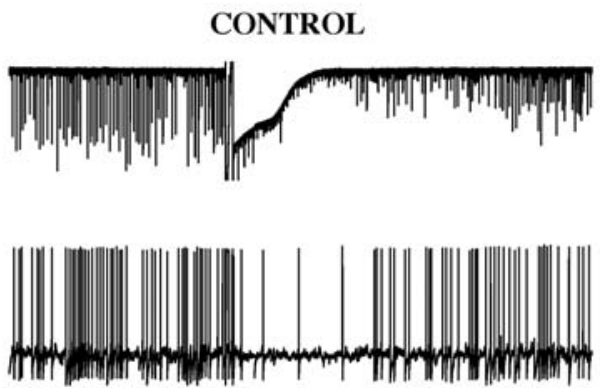

$\mathbf{C}$

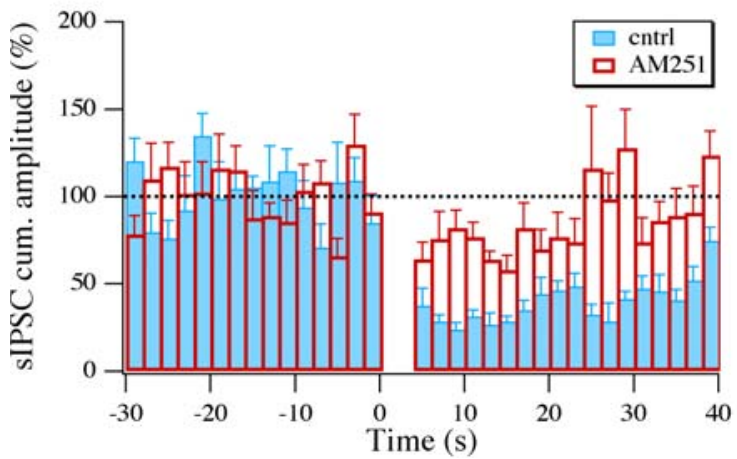

$\mathbf{B}$
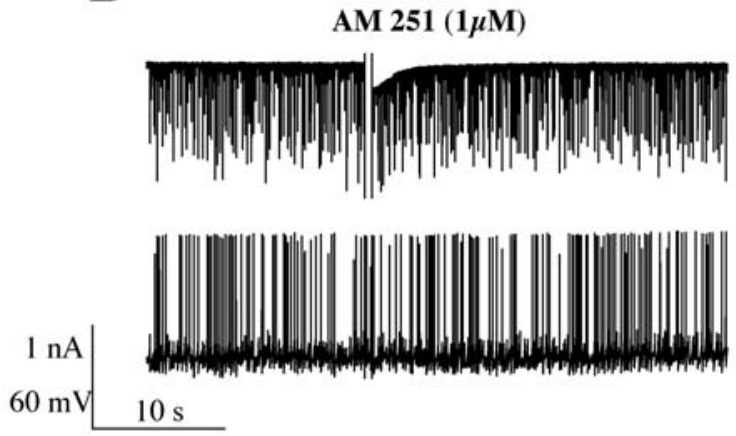

D

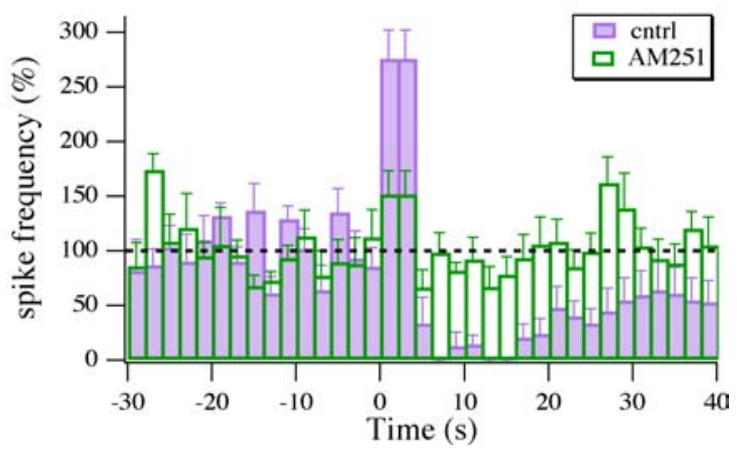

Figure 6.18: In A and B, upper traces are from a postsynaptic Purkinje cell, lower ones are from a synaptically connected stellate cell recorded in current clamp. In this experiment, in control conditions (one trial shown in A) PC depolarization inhibited the interneuron firing frequency to $15.37 \%$ of the pre DSI period while the SIPSC DSI amounted to 70.15\% ( $n=4$ DSI trials). In AM-251, the DSI of SIPSC was $26.10 \%$ and the spike frequency was only reduced to $82.5 \%$ of control $(n=5$ trials); thus, both effects were primarily mediated by CB1Rs. $C$ and D show the time course of firing rate (C) and SIPSC cumulative amplitude (D) for the control and test (AM-251) DSI trials in this pair as indicated ( $t=0$ corresponds to PC depolarization). 

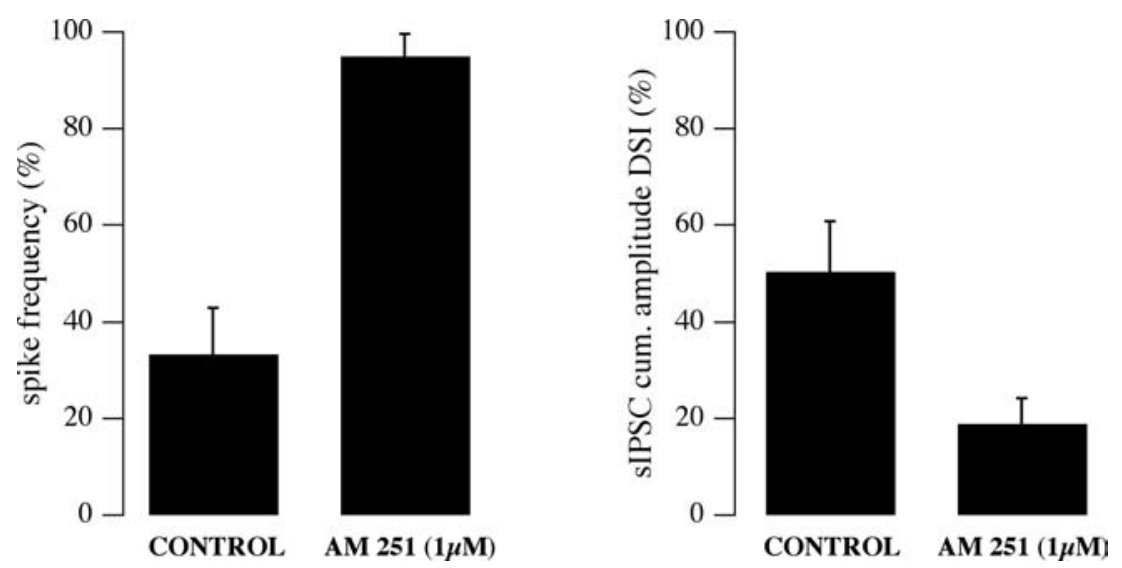

Figure 6.19: The CB1R specific antagonist AM-251 cancels the inhibitory effect of Purkinje cell depolarizations on the spike frequency of molecular layer basket/stellate cells (left bar graph) and reduces the DSI of sIPSCs in Purkinje cells (right). Data have been averaged for 5 paired recordings. In each case, distances between cell bodies were less than $30 \mu \mathrm{m}$ and in two experiments a synaptic connection was present (113.03 pA and $65.56 \mathrm{pA}$ were the average amplitude for evoked currents); see text for further details. Differences between control and AM-251 are highly significant in both panels $(p<0.05$, Wilcoxon paired rank test).

quantitatively show the action of AM-251 on the DSI of sIPSCs (C) and of the spike frequency for this experiment (D). Out of the 5 experiments where this protocol was followed, 3 were synaptically not connected pairs and 2 connected ones and the average intersomatic distance was $18.88 \mu \mathrm{m}$ (range 12.8 to $27.2 \mu \mathrm{m}$, thus all cases were comprised in the region of maximal expression of this phenomenon); Fig.6.19 shows that spike frequency was depressed to 33.38 $\pm 9.61 \%$ during DSI in external, control BBS (range $16.67 \%$ to $59.07 \%$ ) while it remained up to $94.92 \pm 4.64 \%$ after application of AM-251 in the bath (range $82.50 \%$ to $110.53 \% ; \mathrm{p}<<0.05$ ). AM-251 also decreased the DSI of sIPSCs from $50.28 \pm 10.47 \%$ to $18.90 \pm 5.31 \%$ ( $<<0.05$ ). These experiments show that endogenous cannabinoids have both a direct inhibitory effect on synaptic transmission, as shown in the previous paragraphs, and, importantly, that they exert their inhibitory action also by damping the firing frequency of presynaptic GABAergic interneurons.

\subsubsection{CB1R activation by WIN 55, 212-2 inhibits interneuron firing}

Further evidence in favour of a control by CB1Rs of the spiking activity of these cells has come from another series of experiments I have performed; current clamp recordings in perforated patch were done in single interneurons, which induced to fire by injecting small constant 
currents (maximally $\pm 2 \mathrm{pA}$ ), when necessary. After a minimum 5 s-long control period of stationary firing, the CB1R agonist WIN 55, 212-2 (1 or $5 \mu \mathrm{M})$ was applied in the bath for several minutes (range: from 10 to 20 minutes) to assess changes in the cell activity. In some experiments, the CB1R antagonist AM-251 (500 nM or $1 \mu \mathrm{M})$ was added to the bath BBS to verify the actual CB1R specificity of the agonist action. Values referring to drug action have been calculated over minimum 4 minutes in the period of maximal effect of the agent, which varied from one experiment to another (from 3 to $10 \mathrm{~min}$ after application); this fact was likely due to the great lipophilicity of these molecules and, thus, to problems connected with their full penetration into the sliced tissue.

One such experiment is shown in Fig.6.20; the lower graph shows the time course of the interneuron spike rate: each triangular symbol (black for control, red for WIN 55, 212-2, blue for AM-251) represents the average firing for a single $2 \mathrm{~s}$ bin; these bins were then averaged over 1 minute to give the values represented by the yellow dots. The upper voltage traces are $5 \mathrm{~s}$ sweeps from the three different experimental conditions, correspondingly with the aforementioned color code. WIN 55, 212-2 led to a significant inhibition of firing, which remained constant until AM-251 led to a recovery of the spike rate, which actually overshot the control frequency; this confirms the involvement of the CB1 receptors in the phenomenon.

For 7 out of 9 interneurons, for which this protocol was followed, GABAa receptors were continuosly blocked with bicuculline $(10 \mu \mathrm{M})$ to reduce firing variability; the use of bicuculline did not lead to significant changes in the spike rate, thus the 9 cells were pooled together. On average, WIN 55, 212-2 decreased the spike rate to $51.66 \pm 9.69 \%(n=9$; range: $0 \%$ to $91.10 \%)$ of control values, which is significantly less than the effect on spiking in paired recordings for the smaller intersomatic distance described above. In 6 out of 7 cells, where AM-251 was applied following the agonist, spike frequency returned to higher values than in control; the averaged value was $220.30 \pm 45.93 \%$ ( $n=7$; range: $68.54 \%$ to $450.61 \%$ ). Thus, CB1R activation itself leads to the inhibition of basket/stellate cell firing and, furthermore, these experiments suggest a basal level of activation of these receptors in cerebellar slices.

\subsubsection{What mechanisms for the CB1R-induced inhibition of firing?}

At the moment of the writing of this thesis, I am trying to characterize the possible targets of endogenous cannabinoids, which might be responsible for the decrease of basket/stellate cell spike rate. 

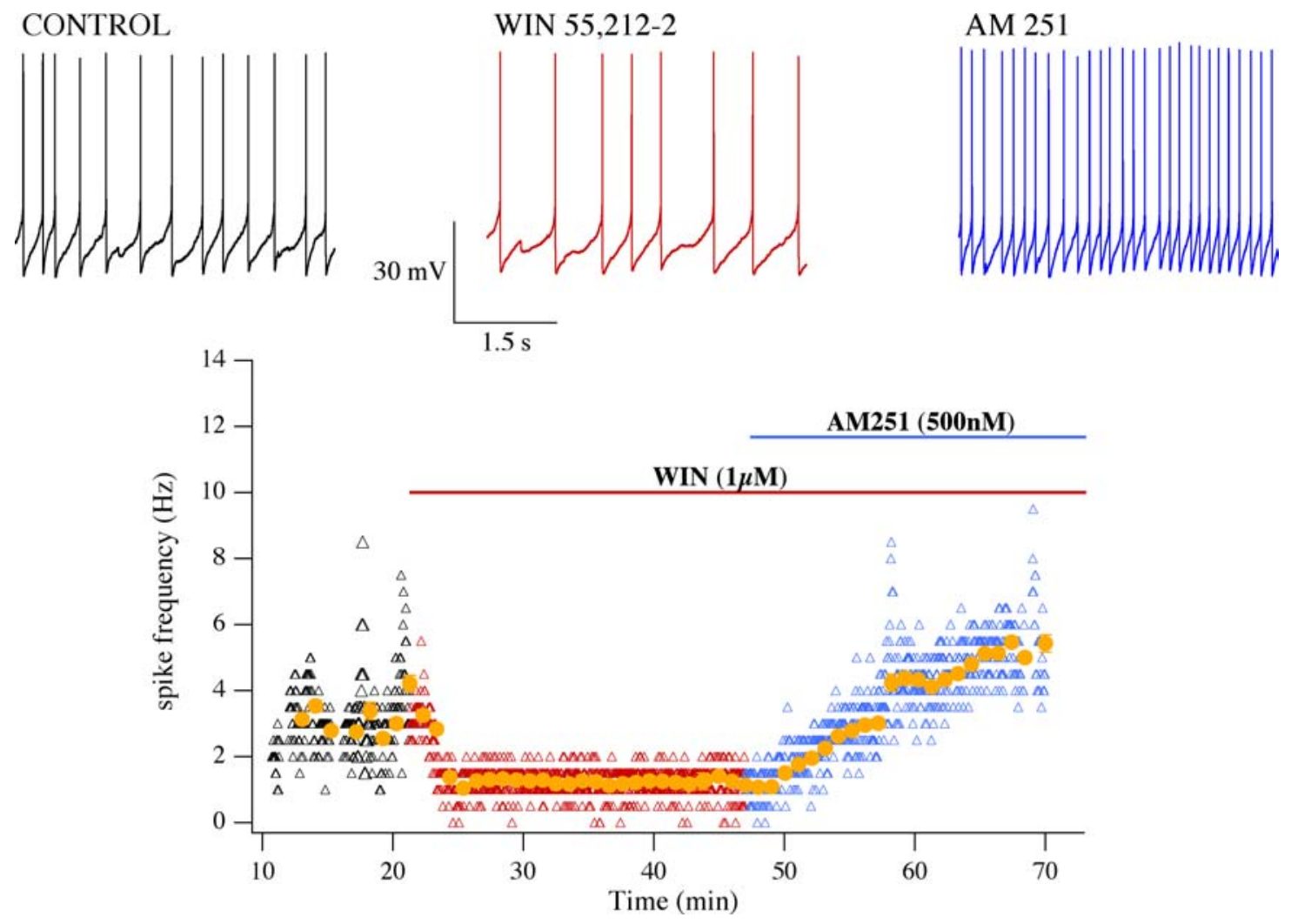

Figure 6.20: The lower graph shows the spike rate time course of a current-clamped stellate cell, recorded through the perforated patch. $+1 \mathrm{pA}$ was injected throughout the experiment; after a control period, in which the cell spiked at $3.17 \pm 0.069 \mathrm{~Hz}$, between 15 and 20 minutes after the application of WIN (added where shown by the corresponding horizontal bar) firing was reduced to $1.22 \pm 0.030 \mathrm{~Hz}(61.52 \%$ inhibition); finally, the CB1R antagonist AM-251 was added and this induced a recovery in the spike rate up to $5.23 \pm 0.073 \mathrm{~Hz}$, which corresponds to $164.98 \%$ of control; this suggests a basal activation of the receptor in the slice. Each triangular marker corresponds to the average spike frequency for one $2 \mathrm{~s} \mathrm{bin}$; bins were then grouped into 1 min sets of data and averaged, thus giving the time course of the values here depicted by yellow, full circles. The upper traces are $5 \mathrm{~s}$ samples of the cell membrane potential, from left to right: in control (black), in WIN (red) and in AM (blue). The color code is same as for the small markers in the lower graph. 
A

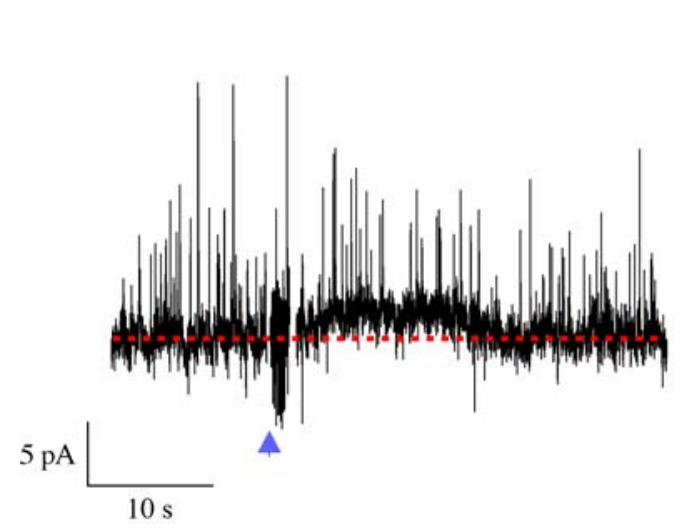

B

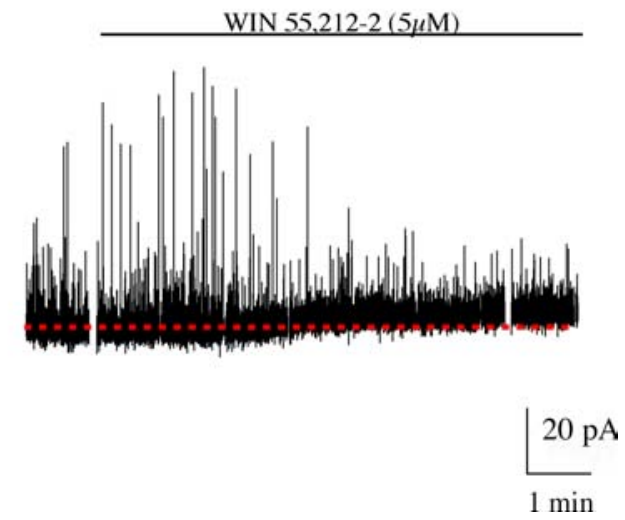

Figure 6.21: Both Purkinje cell depolarizations and exogenous activation of CB1Rs induce an outward current in basket/stellate cells. Traces in A and B are from the same low stellate cell. In A, a synaptically connected Purkinje cell was depolarized after a control period for $1 \mathrm{~s}$ to $0 \mathrm{mV}$, in correspondence with the blue arrow on the interneuron trace: this induced the DSI of sIPSCs in the Purkinje cell (not shown) and a reversible change in the holding current of the interneuron (lower trace; see the shift of the holding current from the control value represented by the red doted line). Notice the increase in gap junctional spikelets in the interneuron recording during depolarization (the small downward deflections). The interneuron was voltage-clamped at $57 \mathrm{mV}$ and the two cell bodies were $77 \mu \mathrm{m}$ distant. In B, WIN55,212-2 changed the holding current and, at the same time, decreased the frequency of sIPSCs. The gaps separating the trace samples are dead times between consecutive recording protocols. The red line represents the average control holding current.

I have obtained very preliminary results, which suggest that at least two important conductances might be modified by CB1R activation, in such a way as to change the threshold for action potentials.

First of all, in voltage clamp experiments, both the application of the exogenous CB1R agonist WIN 55, 212-2 and the depolarization of Purkinje cells induced the appearance of an outward current (or, alternatively, the disappearance of an inward current) in the GABAergic interneuron. In the case of PC depolarizations, this conductance was modified transiently and the holding current returned to control levels with a time course similar to that of spike inhibition. Fig.6.21A shows such an outward current in a voltage-clamped interneuron induced by the depolarization (at the arrowhead) of a synaptically connected Purkinje cells (trace not shown); notice also the increase in the small downward deflections (gap junctional spikelets) during the DSI induction protocol. In Fig.6.21B, the application of WIN 55,212-2 to the same interneuron 
led to a few pA change of the holding current; a few $\mathrm{pS}$ change in membrane conductance could in principle be enough to lead to a dramatic change in the firing rate, given the extremely high input resistance of basket/stellate cells $(>1 \mathrm{G} \Omega$ ). In both $\mathrm{A}$ and $\mathrm{B}$, the control holding current is indicated by the dashed red line.

The identity of this outward current has several possible candidates; Kreitzer et al., 2002, suggested that the upregulation of an inwardly rectifying potassium conductance might generate it. Consistent with our results, this hypothesis will be discussed later.

On the other hand, we considered the possibility that Ih, the hyperpolarization activated inward conductance, might be a target, since its importance in regulating neuron firing rate in many systems and the regulation of its activation curve by intracellular concentrations of cAMP. Thus, in another series of preliminary experiments, the pharmacological block of Th actually led to a promising decrease in the effectiveness with which PC depolarizations affected firing (data not shown). Altogether, these new results suggest that Ih might be downregulated through a shift in its activation curve towards more hyperpolarized potentials and, at the same time, that a second conductance, most likely a potassium current, might be activated. The combined effect would be the powerful silencing of interneurons previously characterized in paired recordings.

\subsubsection{The increase in firing rate during Purkinje cell depolarization: a "true" or an artefactual effect?}

The effect of Purkinje cell depolarizations on the firing frequency of basket/stellate cells is actually biphasic. The CB1R-mediated inhibition developed with some delay after the end of the voltage protocol: this was in the order of some seconds.

Typically, between 100 and $200 \mathrm{~ms}$ after starting the depolarizing pulse, the spike frequency dramatically increased to levels which were much higher than in control. Considering only the cell pairs with intrasomatic distances smaller than $32 \mu \mathrm{m}$, presynaptically connected interneurons reached an average spike frequency of $415.29 \pm 187.04 \%(n=13$; ranging from $74.68 \%$ to $2611.06 \%$ ) while not connected interneurons came to fire at $174.13 \pm 59.66 \%$ ( $\mathrm{n}=9$; range: $85.97 \%$ to $643.927 \%$ ); the difference is statistically significant ( $\mathrm{p}=0.05$; Mann-Whitney test; see Fig.6.22Ab, which is the same graph as in Fig.6.11, but on different scales to highlight this phenomenon). Fig. 6.22Aa shows an exemplar DSI trial for one experiment, in which this biphasic regulation of firing can be appreciated following the DSI induction protocol, whose onset is indicated by the black arrowhead. 
One immediate explanation for the acceleration in firing might come from a depolarizing action of cesium on the interneuron membrane. The postsynaptic intracellular solution contained cesium as main cation; although cesium greatly reduces the conductance of potassium channels, it is possible that, following depolarizations, cesium may exit the Purkinje cell through these channels in sufficient amount to depolarize surrounding cells.

I have therefore performed experiments where cesium was replaced by N-methyl-D-glucamine (NMDG), an impermeant cation, and where, in addition, the aspecific potassium channel blocker TEA $(10 \mathrm{mM})$ was present in the pipette. In this way, any artefact coming from partially conducting potassium conductances could be excluded. With this postsynaptic solution, DSI could be reliably induced and, furthermore, the regulation of firing remained biphasic as in the experiments performed with cesium. Several cell pairs $(n=5)$ have so far been performed in which this phenomenon was still visible, both for full spikes and for gap spikelets. Fig.6.22Ba shows one DSI trial from one such paired recording.

Several hypotheses explaining the facilitatory effect can be proposed. It will be particularly important to establish any possible role of cannabinoids and CB1Rs. A reliable finding in the experiments where cesium has been replaced with NMDG was the development of an inward current in interneurons in response to a DSI protocol. This current had a time course similar to that of the firing increase and could, thus, mediate it. Two examples are shown in Fig.6.22Bb; these traces have been taken while voltage clamping the presynaptic interneuron at different voltages, indicated in the figure. Surprisingly, the inward current appears to increase with hyperpolarized potentials; this could be an important indication about its identity.

At the time of the writing of this thesis, experiments are being performed to determine the molecular mechanisms underlying these observations. 
A

a

postsynaptic CesiumGluconate

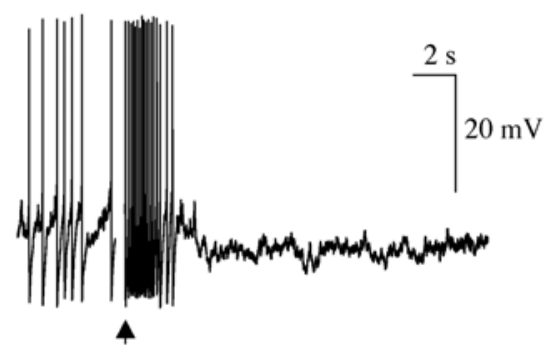

b

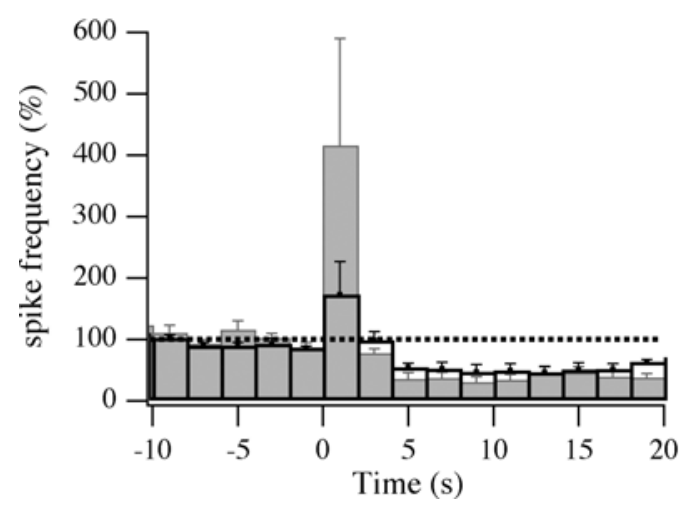

B

a postsynaptic NMDG-Cl + $10 \mathrm{mM}$ TEA

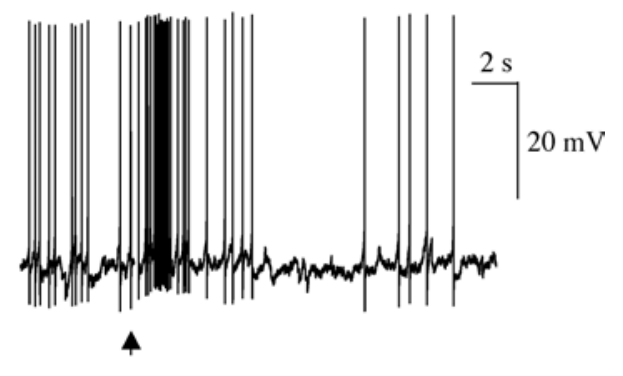

b
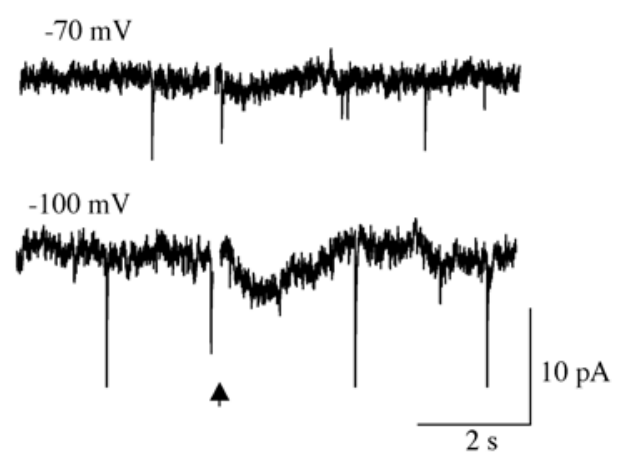

Figure 6.22: Biphasic control of interneuron spike rate by Purkinje cells.The first clear effect of DSI induction protocols on interneuron is an acceleration in firing. Aa shows a typical example, where between 100 to $200 \mathrm{~ms}$ after the onset of the depolarization the interneuron had a burst of action potentials, which then faded out leaving room to the slower inhibitory period. For this synaptically weakly connected pair (average eIPSC amplitude was $16.76 \pm 1.95 \mathrm{pA} ; \mathrm{n}=54$ ), firing increased to $543.34 \%$ of control during the pulse and was inhibited to $11.26 \%$ between 4 and $10 \mathrm{~s}$ after the end of the depolarization. Cell bodies were $22 \mu \mathrm{m}$ distant. Ab shows the same graph as in Fig.6.11, but with axis coordinates appropriately enlarged to show the big increase in firing during the pulse and the significant difference between connected (filled bars) and not connected (black lined bars) pairs. Only intersomatic distances smaller than $32 \mu \mathrm{m}$ were considered. Part Ba shows that spike rate facilitation did not artefactually depend upon exit of cesium from the postsynaptic cell during the DSI protocol. The Purkinje cell was recorded from with NMDG-Cl replacing CsGluconate in the intracellular solution plus $10 \mathrm{mM}$ of the aspecific $\mathrm{K}^{+}$channel blocker TEA. 1 s-long depolarizations (with onset for this trial shown by the arrowhead) increased firing to $356.99 \%$ of control; inhibition then took over, damping the spike rate to $37.29 \%$ of control. DSI of sIPSCs was $55.22 \%$, showing that the Purkinje cell was able to produce cannabinoids also with this internal solution. Cells were not synaptically connected and cell bodies were separated by $34 \mu \mathrm{m}$. Finally, Bb illustrates a common finding in NMDG experiments: when voltage-clamped during the DSI protocol, interneurons showed a transient increase in the holding current, that returned to control levels with time course similar to the effect on firing and that, surprisingly, increased with hyperpolarization of the membrane potential. Traces are from the same paired recording of part Ba. 


\section{Chapter 7}

\section{DISCUSSION}

A thorough characterization of DSI has been performed.

The use of the perforated patch configuration in paired recordings has allowed the description of a series of parameters of synaptic transmission, which have confirmed the presynaptic nature of DSI. The stability of transmission for long experimental periods, the control of the presynaptic fire rate and, finally, the preservation of the intracellular milieu preventing dialysis-induced artefacts: all these properties provided by this technique have led to the improvement of our knowledge on this form of retrograde modulation.

The nature of the retrograde messenger(s) was established and, as a consequence, an impressive spectrum of actions of cannabinoids on transmission was found, from inhibition of basic synaptic mechanisms to a biphasic modulation of the firing frequency.

Finally, all of these effects were shown to impinge on a wide network of electrically and synaptically connected GABAergic interneurons, whose complex level of interplay was also hinted at.

All these aspects are approached and discussed in the following sections.

\subsection{The various components of DSI: a quantitative evaluation}

The impact of DSI on different forms of GABAergic synaptic transmission onto Purkinje cells was studied: miniature IPSCs, spontaneous IPSCs and evoked synaptic currents. It was, thus, possible to establish that this inhibitory phenomenon affects transmission at all levels: from the generation of action potentials to action potential-induced calcium transients and, finally, down to the release machinery. 
Using the whole-cell technique in Purkinje cells, the DSI of sIPSCs was analyzed; its various components were dissected: sIPSCs are, indeed, an heterogeneous mixture of miniature IPSCs, which were isolated through the use of TTX, and of evoked IPSCs, which are induced by presynaptic action potentials and were studied in paired recordings.

What is the contribution of mIPSCs on the overall population of sIPSCs? My data would indicate that most of the events recorded as spontaneous currents were action potential-independent mIPSCs: the ratio of mIPSC over sIPSC frequency was $0.737(8.20 \mathrm{~Hz}$ versus $11.13 \mathrm{~Hz})$. In my view, these values are actually unlikely; in interneurons for which 0 or maximal $+1 \mathrm{pA}$ were injected in current clamp conditions (so that the actual basal firing frequency was not excessively overestimated), the average spike frequency was $3.81 \pm 1.11 \mathrm{~Hz}$ at room temperature $(\mathrm{n}=8$; see Fig.6.20). This value was confirmed in other series of experiments performed independently in the laboratory (J. Chavas, personal communication). According to this value, then, there would be on average only one active presynaptic cell per Purkinje cell contributing to the population of sIPSCs. This is unlikely. A possible explanation for this result comes from the fact that these values were obtained in independent experiments: sIPSC and mIPSC frequencies were never compared during the same experiment and mIPSC frequencies were extremely variable between cells, with a particularly strong dependence on the animal age. This is why I consider as a more reliable evaluation what was done in another series of experiments in our laboratory (Gonzalez, Llano and Marty, unpublished data), where TTX was added for a same Purkinje cell following a control period and the amount of mIPSCs was truly compared to that of the sIPSCs for a same recording; these studies have given a different ratio: 0.47 for the frequency and 0.21 for the cumulative amplitude (Gonzalez, Marty and Llano, unpublished data).

How does this heterogeneous population of events influence the total amount of DSI induced by Purkinje cells? One first hint comes from a quantitative comparison of the DSIs of the various components of transmission. It can be noticed that while the DSI of mIPSCs (43.3\%) was statististically different from that of sIPSCs (64.2\%) and of eIPSCs (68.8\%), these last 2 values were not. This may seem paradoxical. We argued earlier that mIPSCs represent a high percentage of the overall population of synaptic events recorded as SIPSCs and that mIPSCs are comparatively less affected than sIPSCs by DSI. This implies that action potential-dependent sIPSCs must be even more inhibited than the total population of sIPSCs during DSI. But this is not found by measuring the DSI of eIPSCs in paired recordings. The response to this apparent contradiction comes from the inhibition of presynaptic firing by endocannabinoids. Infact, this effect does not 
appear in our paired recordings with presynaptic voltage clamp, where the presynaptic stimulus was largely suprathreshold, and reliably gave rise to somatically recorded action currents even during DSI. While action potential-dependent currents contribute to the cumulative amplitude of the sIPSCs through the product of the presynaptic average firing rate and of the average amplitude of the evoked currents, the DSI of eIPSCs describes only the effect on the latter factor. The DSI of sIPSCs, as measured in Fig.5.1, can indeed be accounted for by its various components as derived from mIPSC and eIPSC measurement and from the characterization of the DSI of firing. We can justify this statement as follows:

\section{Frequency Analysis:}

The frequency of spontaneous IPSCs, $f_{s}$, is the sum of the frequency of sIPSCs occurring independently of presynaptic action potentials, $f_{m}$, and of the frequency of events triggered by presynaptic action potentials, $f_{A I}$ :

$$
f_{s}=f_{m}+f_{A I}
$$

The former frequency is assumed to be the same as the frequency of mIPSCs measured in the presence of TTX. The latter frequency is the product of the frequency of presynaptic action potentials, $f_{A P}$, with the probability of having no transmission failure. Calling FR the failure rate, we obtain:

$$
f_{A I}=f_{A P}(1-F R)
$$

We call $\% f_{m}$ the percentage of mIPSCs among sIPSCs:

$$
\% f_{m}=\frac{f_{m}}{\left(f_{m}+f_{A I}\right)}
$$

The remaining percentage of mIPSC frequency during DSI is:

$$
1-D S I_{m}=\frac{f_{m, D S I}}{f_{m, c t l}}
$$

where $f_{m, D S I}$ and $f_{m, c t l}$ are respectively the values of $f_{m}$ during DSI and in the control. Likewise the remaining percentage of action potential frequency is:

$$
1-D S I_{A P}=\frac{f_{A P, D S I}}{f_{A P, c t l}}
$$


and the remaining percentage of sIPSC frequency is:

$$
1-D S I_{f, s}=\frac{f_{s, D S I}}{f_{s, c t l}}
$$

Applying eq. (1) to both control and DSI conditions yields, in combination with eq. (6):

$$
1-D S I_{f, s}=\frac{\left(f_{m, D S I}+f_{A I, D S I}\right)}{\left(f_{m, c t l}+f_{A I, c t l}\right)}
$$

inserting eq. (2) and (3) we obtain:

$$
1-D S I_{f, s}=\left[f_{m, D S I}+f_{A P, D S I}\left(1-F R_{D S I}\right)\right] \frac{\% f_{m, c t l}}{f_{m, c t l}}
$$

By inserting eq. (5), and applying eq. (2) to the control situation, we obtain:

$$
1-D S I_{f, s}=\left\{f_{m, D S I}+\left[\left(1-D S I_{A P}\right) \times f_{A I, c t l} \frac{\left(1-F R_{D S I}\right)}{\left(1-F R_{c t l}\right)}\right]\right\} \frac{\% f_{m, c t l}}{f_{m, c t l}}
$$

This can be rearranged to yield:

$$
1-D S I_{f, s}=\% f_{m, c t l}\left(1-D S I_{m}\right)+\left[\left(1-\% f_{m, c t l}\right)\left(1-D S I_{A P}\right) \frac{\left(1-F R_{D S I}\right)}{\left(1-F R_{c t l}\right)}\right]
$$

inserting in this equation the proper parameter values

$\% f_{m, c t l}=0.47$ (J. Gonzalez, A. Marty and I. Llano, unpublished)

$D S I_{m}=0.452$

$D S I_{A P}=0.20$ (Kreitzer et al., 2002)

$F R_{D S I}=0.20$

$F R_{c t l}=0.012$

gives

$D S I_{f, s}=40.0 \%$

which is close to the experimental value from Fig.5.1 (39.9\%).

For this calculation, we have decided to use the value of 0.20 for the DSI of the spike frequency reported by Kreitzer et al., instead of our $50.41 \pm 8.25 \%(n=22)$; this value was obtained from all the paired recordings with a functional synaptic connection, for which the effect on firing of Purkinje cell depolarizations was studied. The reason is that the sample of cells we considered was clearly biased towards small intrasomatic distances: 17 out of 22 pairs were at distances 
smaller than $50 \mu \mathrm{m}$, and 13 out of 22 at less than $30 \mu \mathrm{m}$ (see Fig. 6.10). Given the space constant of $33.88 \mu \mathrm{m}$, which described the decrease of the inhibition with intersomatic distance, the average inhibition was likely overestimated by using this sample. We, then, decided to use the smaller estimation, which has been taken from the graph in Figure 4 of Kreitzer et al., 2002: here, they represent the time course of the phenomenon for all their pairs $(n=22)$ at distances smaller than $100 \mu \mathrm{m}$.

\section{Cumulative amplitude analysis:}

Similar equations can be derived for cumulative amplitudes. In this case, failures do not need to be considered, because the percentage of reduction of eIPSCs, $D S I_{e}$, already includes transmission failures. The calculations yield:

$$
1-D S I_{C, s}=\% C_{m, c t l}\left(1-D S I_{m}\right)+\left[\left(1-\% C_{m, c t l}\right)\left(1-D S I_{A P}\right)\left(1-D S I_{e}\right)\right]
$$

where $D S I_{C, s}$ and $\% C_{m, c t l}$ are respectively the percentage of reduction of the cumulative amplitude of sIPSCs, and the proportion of mIPSCs in the control cumulative amplitude. The numerical value of $\% C_{m, c t l}$ is 0.21 , that of $D S I_{e}$ is $68.8 \%$ and of $D S I_{m}$ is, for the cumulative amplitude, $43.34 \%$; hence eq.(11) gives: $D S I_{C, s}=68.8 \%$, which is close to the measured value of $64.2 \%$.

To calculate the share of the total inhibition which is due to the various components of DSI, we note that, out of $1 \mathrm{nA}$ of cumulative amplitude of sIPSCs, mini reduction amounts to $0.452 \times$ $0.21=0.0949 \mathrm{nA}$. The reduction due to action potential suppression amounts to $0.2 \times 0.79=$ $0.158 \mathrm{nA}$. Finally the reduction due to eIPSCs following presynaptic firing is $0.688 \times 0.8 \times$ $0.79=0.435 \mathrm{nA}$. The proportions of the respective reductions are $13.8 \%, 23.0 \%$ and $63.2 \%$.

The excellent match between predicted and calculated parameters for the DSI of sIPSCs indicates that this analysis correctly identifies the various components of DSI and their respective weights. Moreover, it confirms the importance of having performed paired recordings: the knowledge of failure rates and of the percentage reduction in evoked currents during DSI have allowed the prediction of the effect of DSI on firing, and, besides its characterization, the assessment of its precise share in the overall inhibition.

A final, interesting outcome of this analysis is the fact that action potential-independent IPSCs contribute to about $50 \%$ of the residual sIPSCs at the peak of DSI, compared to $21 \%$ in the control (cumulative amplitude data). Thus, the relative low sensitivity of these synaptic currents to DSI translates into an increase of their weight to very significant proportions during 
DSI periods.

\subsubsection{Further arguments for the existence of multiple mechanisms underlying DSI}

The previous evaluation shows that the main contribution to DSI is quantitatively given by the reduction of the amplitude of evoked IPSCs downstream somatic action potentials; thus, correspondingly, the relative sensitivity of miniature currents to DSI renders this component the source of most of the remaining GABAergic input onto Purkinje cells.

What are the possible mechanisms leading to this important reduction in evoked transmission? The experiments depicted in Fig.6.7 show that full CB1R activation led to a decrease of about $25 \%$ in axonal calcium transients in response to 4 somatically induced action potentials. This is likely to be originated by a direct inhibition of calcium conductances by CB1R activation as in hippocampal DSI (Wilson et al., 2001). But this may not be all, as far as cerebellar DSI is concerned.

First, it is important to state that, although the source of agonist was in this case exogenous, arguments exposed in section 7.4 on the similarity of the quantitative effects on release between DSI and saturating doses of WIN55,212-22 should ensure that these manipulations mirrored the real situation during DSI.

Secondly, assuming that WIN55,212-22 inhibited calcium transients elicited by 1 action potential to the same amount, this reduction in calcium could explain the dramatic decrease in eIPSC amplitude from paired recordings (DSI amounts to $84.87 \pm 3.59 \%$ in the pooled $n=11$ experiments for the data of Fig.6.3 and Fig.6.4) only if transmission depended upon calcium with a power coefficient between 6 and 7. This value is remarkably greater than so far described in the literature (Neuromuscular Junction: Dodge and Rahamimoff, 1967; Squid Giant synapse: Augustine and Charlton, 1986; Calix of Held: Bollmann et al., 2000; Schneggenburger et al., 2000); it appears, thus, unlikely that the reduction in calcium per action potential could account for the whole depression.

At least a second mechanism should contribute to the DSI of eIPSCs; the decrease in mIPSC frequency indicated that cannabinoids were able to influence release probability also acting on pathways unrelated to the $\mathrm{Ca}^{2+}$-dependent steps of transmission. Be they upstream (a modulation of the number of vesicles in the readily releasable pool?) or downstream (a direct modulation of synaptic proteins?) calcium entry, these steps were presumably able to modulate also the action potential-dependent part of release. 
Moreover, it must not be forgotten the action of cannabinoids on the firing frequency of interneurons; the small and not yet identified outward current induced in interneurons by cannabinoids and Purkinje cell depolarizations could cause changes in the shape of the action potentials and, thus, in the calcium levels reached during stimulation.

Consistently, in a series of experiments which are not described in this dissertation, the use of a cesium-based presynaptic solution to stimulate interneurons through the perforated patch led to a strong reduction in DSI. Cesium blocks and/or reduces potassium channel conductances, thus also dramatically increasing synaptic transmission from terminals by potentiating the inflow of calcium; the full exchange of intracellular potassium with the pipette cesium (to which AmphotericinB is permeable) could be assessed by the change in the properties of the evoked currents (amplitude, variability and kinetic parameters); these were consistent with those described for similar experiments with presynaptic whole-cell by Vincent and Marty, 1996. Importantly, the reduction of extracellular calcium brought back transmission to potassium-like levels and increased the amount of DSI, but not back to control values; in this way, the saturation of transmission could be identified as responsible for a part, but not all, of the decrease of DSI by presynaptic cesium.

These experiments strongly support the view that an upregulated potassium conductance might contribute to some, but not all, the DSI of evoked transmission.

In my view, the likeliest possibility sees in the regulation of calcium and potassium channels and in a direct action on release machinery the multiple origin of this striking effect of cannabinoids on action potential-dependent release. The possible molecular identities of these targets of the cannabinoid system are described in detail in the introduction section devoted to the cannabinoid system.

\subsection{Paired Pulse Ratio: comparison with previous work}

Contrasting reports have been published on the behaviour of the PPR during DSI in the hippocampus. No change (Alger et al., 1996) and an increase (Wilson and Nicoll, 2001) in the ratio have been described.

There are at least two possibilities to explain these diverging results; the first originates from the approach used in these studies to evoke GABAergic currents in CA1 pyramidal cells: extracellular stimulation elicited action potentials in cells, which then synapsed onto the recorded 
cell. This is a widely used technique, when paired recordings are not possible or when they are successful at too low a probability. Weak points associated with this experimental procedure are the following: the number and the identity of the stimulated cells cannot be identified and, furthermore, their firing pattern is not controlled between consecutive pulses. The amplitude of a series of currents evoked at high frequency (like for the measurement of the PPR) might, thus, be influenced by the basal firing activity of the stimulated cells; this could itself be variable between laboratories as a function, for example, of different protocols of slice preparations.

A second explanation might come from the way the PPR is measured: calculating the PPR as the ratio between average PPR from single sweeps can lead to an overestimation, especially in conditions where evoked currents are small and/or extremely variable (Kim and Alger, 2001). The bias given by the eIPSC variability can be overcome by considering the ratio between average second and first eIPSCs from a group of many currents. Unfortunately, neither of the groups contributing the quoted reports explained how the PPR was calculated from their data, so that a difference coming from this cannot be excluded.

For what concerns this thesis, both problems have been avoided: the PPR was calculated as the ratio between average current amplitudes and paired recordings allowed the precise control of the presynaptic spike rate. The results were straightforward: the PPR increased during DSI for all the paired cells where it was tested, in line with a presynaptic expression of DSI.

One more point concerning these data deserves further discussion; at $50 \mathrm{hz}$, the percentage PPR in control was $95.64 \pm 5.90 \%(n=11)$. Moreover, in experiments performed for other purposes (see Fig. 6.3), the PPR was $101.90 \pm 7.1 \%(\mathrm{n}=5)$ at $20 \mathrm{hz}$ and not statistically different from $100 \%$ at $30 \mathrm{~ms}$ interpulse interval (M. Galante, personal communication). Thus, throughout a wide range of high stimulation frequencies, this synapse is neither facilitating nor depressing; this is in contrast with previous reports, which described a depressing behaviour (i.d.: PPR < $100 \%$ in the rat: Pouzat and Hestrin, 1997 and in the mouse: Caillard et al., 2000) and with the high release probability of these connections at this developmental stage (Pouzat and Hestrin, 1997).

In my view, the difference is to be justified by the perforated patch versus whole cell recording configuration, upon which these 2 previous reports were based; as shown in Caillard et al., 2000, the absence of intracellular components and in particular of soluble calcium buffering proteins like parvalbumin can have dramatic effects on the dynamic behaviour of these synapses. Moreover, these authors showed that the PPR value could be modulated by the calcium buffering 
power of the intracellular solution; this supports the thesis that intracellular dialysis has dramatic effects on the dynamics of these synapses.

The perforated patch configuration allows to circumvent all these artefactual contributions given by intracellular dialysis, thus providing, in my view, data which more likely represent the "true" physiological conditions.

\subsection{Increased failure rate during DSI}

Failures can be unequivocably detected in paired recordings; in my case, a noise threshold was set for detection of the evoked currents in the analysis program and further visual inspection of each single trace confirmed the occurrence of a failure.

DSI has already been associated with failures of extracellularly evoked currents, when low intensity stimulation is used, or with failures of some of the components, which can be detected by inflexions on the rising phase of the currents (Vincent et al., 1992 and Alger et al., 1996).

A change in failure rate cannot easily be explained by postsynaptic arguments: a postsynaptic mechanism would yield a scaling of eIPSC amplitudes without the complete disappearance of the currents. Nevertheless, a consistent mechanism would be a complete silencing of the array of receptors, at all the active zones between the 2 cells. In the case of DSI, this should happen within a few seconds. No reports of such a series of event has been so far presented; insertion and withdrawal of receptors from postsynaptic membranes represents the most widely accepted hypothesis for the expression of LTP and LTD in the hippocampus (see Malenka and Malinow, 2002); however, these phenomena reach maximal expression and have in general a time course spanning much longer time intervals than DSI. It, thus, appears unlikely that a contribution from receptor trafficking may account for the inhibition during DSI.

So, the increase in failure rates in the first minute following the induction protocol is a strong element in favour of a presynaptic expression of DSI. The low failure rate in control conditions is, furthermore, an indication of the high release probability and of the strength of these connections in young animals.

\subsection{An endocannabinoid as retrograde messenger for cerebellar DSI}

The present results indicate that endogenous cannabinoids (endocannabinoids) are the retrograde messengers at inhibitory synapses onto Purkinje cells. This hypothesis is supported by 
several arguments, as follows.

First, endocannabinoids and DSI both act on presynaptic targets. We found that CB1R activation increased the paired-pulse ratio in paired recordings and decreased mIPSC frequency without modifying the quantal size, strongly indicating a presynaptic action of endocannabinoids. This conclusion was further supported by our morphological data showing the absence of the receptor from Purkinje cells or Bergmann glia and by its specific presence on presynaptic structures.

Secondly, the maximum effects of DSI and of CB1R were quantitatively very close. The induction protocol used for this work was chosen to produce maximum DSI (Glitsch et al., 2000); likewise, the WIN 55,212-2 concentration tested $(5 \mu \mathrm{M})$ was far beyond the saturating dose for CB1Rs. In this regard, this high concentration was not likely to give artefactual effects on synaptic transmission: indeed, several reports showed that the synaptic effects of similar, or of even higher, concentrations were fully blocked by application of specific antagonists (see for example: Chan and Yung, 1998; Hoffman and Lupica, 2000; Takahashi and Linden, 2000 and Robbe et al., 2001). Nevertheless, in both cases, GABAergic transmission was not completely inhibited. The percentage maximum effect was clearly different between TTX-sensitive and insensitive release but, in both cases, the amount of residual synaptic transmission was similar for DSI and WIN 55,212-2 inhibition (for eIPSCs: $13.0 \pm 2.6 \%$ of control in DSI experiments, $\mathrm{n}=10$, vs. $10.7 \pm 2.1 \%$ of control in the presence of $\operatorname{WIN} 55,212-2, \mathrm{n}=5 ; \mathrm{p}>0.05$; for mIPSCs: $57.1 \pm 3.6 \%$ of control in DSI experiments, $\mathrm{n}=9$, vs. $44.4 \pm 6.7 \%$ of control in the presence of WIN 55,212-2, $\mathrm{n}=10, \mathrm{p}>0.05$ ). Importantly, this similarity in effect is also a strong element suggesting that CB1Rs were saturated by the endocannabinoids produced by Purkinje cells during DSI.

The third line of evidence is that blocking CB1Rs with the antagonist AM-251, as well as full activation with a saturating dose of WIN 55, 212-2, led to a strong reduction of DSI. This applied both for the modulation of eIPSCs, of mIPSCs and of sIPSCs. These results confirm that DSI and CB1Rs share the same molecular pathway and indicate that CB1R activation is a necessary and sufficient condition for the expression of DSI.

The new conclusion that endocannabinoids account for DSI in our system is in agreement with their involvement in the similar form of transient inhibition of excitatory synaptic currents onto Purkinje cells called DSE (Kreitzer and Regehr, 2001a). Following depolarization of Purkinje cells, endocannabinoids inhibit both IPSCs coming from basket and stellate cells and EPSCs 
originating in parallel fibers and climbing fibers. All of these actions occur with a similar time course and tend to isolate the cell from its synaptic inputs. It remains to be found whether in physiological conditions this cannabinoid-mediated retrograde inhibition can be synapsespecific or whether it is just a mean to scale down the synaptic input onto Purkinje cells indiscriminately. The possible selectivity of cannabinoid action on different forms of synaptic transmission is likely to depend upon the physiological mechanisms leading to the synthesis of these compounds. In this regard, it must be reaffirmed that the $\mathrm{Ca}^{2+}$ sensitivity of cerebellar DSI has been reported to be extremely high (Glitsch et al., 2000): $\mathrm{Ca}^{2+}$ concentration increases in the order of a few hundred nanomolar are enough to elicit DSI while, for example, very strong depolarizations are required to elicit the DSE of climbing fiber input onto Purkinje cells (Kreitzer and Regehr, 2001a).

The conclusions drawn from our experiments are also in agreement with recent reports indicating a similar role for cannabinoids in hippocampal DSI (Wilson and Nicoll, 2001; Wilson et al., 2001; Ohno-Shosaku et al., 2001) and with other studies confirming our findings in the cerebellum (Kreitzer and Regehr, 2001b and Yoshida et al., 2002).

\subsection{What about the glutamate hypothesis?}

The question arises of their compatibility of the above conclusions with the previous data, that led to the suggestion of a role for glutamate or a related substance in cerebellar (Glitsch et al., 1996) and in hippocampal DSI (Morishita et al., 1998).

In the cerebellum, these data included the report of an imitation and occlusion of DSI with DCG IV, an agonist of group II mGluRs, and of an inhibition of DSI in the presence of LAP3, an antagonist of mGluRs. The former effect could be compatible with the proposal of cannabinoids as messenger if the mGluR and CB1R pathways were converging downstream of the receptor level to produce a DSI-like phenomenon; this is a likely possibility, since both CB1 and mGluR2 receptors activate the same pertussin toxin sensitive Gi/o protein subtype: occlusion could then be explained on the basis of competition for common steps of the transduction pathway leading to DSI. Concerning the latter effect (inhibition by L-AP3), it is first to be noticed that the inhibition was less pronounced than with AM-251: the residual DSI of mIPSCs observed in L-AP3 was 59\% of its original value compared to $33 \%$ in the presence of AM-251. Secondly, it should be stressed that L-AP3 is a poorly specific drug. In earlier work, L-AP3 
had to be used at a high concentration $(1 \mathrm{mM})$, and several side effects were found, including a partial agonist action on type II mGlu and on NMDA receptors (Glitsch et al., 1996). Since the broad action antagonist MCPG failed to inhibit DSI (Glitsch et al., 1996), the relatively weak inhibition exerted by L-AP3 could in retrospect be explained by the partial agonist effect on mGluRs, which would then lead to an inhibition of DSI through an occlusion effect similar to that observed with DCGIV, and/or on NMDA receptors, which facilitate transmission and could counteract the inhibitory action by endocannabinoids (Glitsch and Marty, 1999).

Finally, it must noticed that the DSI of mIPSCs (Fig.6.6) and of sIPSCs (Fig.6.19) were not completely canceled by the CB1R antagonist AM-251; 9.10\% and 18.90\% decreases of spontaneous and miniature currents, respectively, were still present after drug application; it has been reported that the mGluR2R specific blocker LY 349415 led to a small, but significant inhibition of DSI of mIPSCs for the same synapses (Glitsch and Jack, 2001) and Purkinje cells have actually been suggested to release glutamate in a calcium-dependent way following activation of group 1 mGlu receptors (Levenes et al., 2001). Thus, it would still be possible that glutamate released together with endocannabinoids from Purkinje cells (or from other sources like glial cells; glial cells can release glutamate in response to small increases in calcium concentrations induced by neurotransmitters: Parpura and Haydon, 2000 and for a review: Haydon, 2001) might lead to a small reduction in release probability through presynaptic group 2 mGluR (localized on basket cell pinceaux: Tamaru et al., 2001).

In contrast with the report by Glitsch and Jack, 2001, though, in our hands LY 341495 did not eliminate the residual DSI of mIPSCs left after CB1R block; furthermore, a depressive action by glutamate through presynaptic ionotropic glutamate receptors, as proposed by a recent report (Satake et al., 2000), could be excluded for the continuous presence of NBQX in all the performed experiments. In view of these results, even a small role of glutamate in mediating DSI appears unlikely.

As for the role of glutamate in the DSI of hippocampal GABAergic currents, the subject requires a small discussion on its own for the importance the relationships between group 1 mGluRs and cannabinoid system might have.

\subsubsection{Glutamate, group $1 \mathrm{mGlu}$ receptors and endocannabinoids}

Indeed, data concerning the involvement of glutamate in hippocampal DSI showed a role for group 1 but not group 2 mGluRs; the situation was parallel to that in the cerebellum: agonists 
occluded DSI and antagonists partially blocked it (Morishita et al., 1998).

Although it had been known for some time that group 1 mGluR activation depressed GABAergic transmission (Gereau IV and Conn, 1995), the involvement of presynaptic group 1 mGluRs was in contrast with the morphological evidence; this showed the presence of these receptors only on postsynaptic membranes, particularly in perisynaptic regions around excitatory terminals (Baude et al., 1993 and Nusser et al., 1994). Group 1 receptors are linked to the pertussis toxin insensitive Gq protein subtype, whose activation leads to the phospholipase C-mediated production of DAG (a precursor of 2-AG), of $\mathrm{IP}_{3}$ and to increases in intracellular calcium concentration from intracellular stores (Abe et al., 1992, Aramori and Nakanishi, 1992). By this way, a direct line connecting group 1 mGluRs with cannabinoid synthesis can be imagined. And, indeed, glutamate is involved in hippocampal DSI, but only as modulatory factor rather than as a retrograde messenger. Activation of mGluR1 receptors leads to the production of endocannabinoids, which inhibit glutamatergic transmission in the cerebellum (Maejima et al., 2001) and GABAergic transmission in the hippocampus (Varma et al., 2001 and Ohno-Shosaku et al., 2002a) through presynaptic CB1Rs. The same mechanisms underlie the group $1 \mathrm{mGluR}$ mediated inhibition of GABAergic transmission in the cerebellum (M. Galante and M. Diana, unpublished observation) and of glutamatergic transmission in the Amygdala (Marsicano et al., 2002), the Nucleus Accumbens (Robbe et al., 2002) and the Striatum (Gerdeman et al., 2002). Importantly, it is still unclear whether increases in intracellular calcium concentration are (Robbe et al., 2002) or are not (Maejima et al., 2001; Ohno-Shosaku, 2002) fundamental for the synthesis of cannabinoids downstream mGlu receptors.

The interaction of this pathway with DSI is extremely intriguing; low concentrations of group 1 mGluR agonists enhance DSI (Varma et al., 2001; Ohno-Shosaku, 2002) while group 1 mGluR antagonists partially block it (Morishita et al., 1998 and Varma et al., 2001). Low agonist concentrations or the basal level of extracellular glutamate, which can reach values in the micromolar range (Lerma et al., 1986; Timmerman and Westerink, 1997), may activate postsynaptic group 1 mGluRs to some extent in basal conditions, "warming up" and upregulating the cannabinoid machinery. The induction protocol of DSI may then fully trigger a machinery already primed for cannabinoids synthesis, thus resulting in enhanced presynaptic inhibition. This would explain the partial effect of group 1 mGluR blockers on hippocampal DSI; moreover, the variability observed in DSI between experiments might derive from the extracellular level of glutamate, which itself may depend on the state of the preparation, the temperature at 
which experiments are performed and the developmental stage of the animals used. Then, the question arises whether CB1Rs are already partially activated in control conditions in Purkinje cells and whether, if it is actually the case, group 1 mGluRs are involved. One report indicates that group $1 \mathrm{mGluRs}$ on Purkinje cells are partially activated by extracellular glutamate in control conditions (Yamakawa and Hirano, 1999). The data from my experiments showed no significant potentiating effect on eIPSC amplitude and on mIPSC frequency after application of the CB1R antagonist AM-251. Moreover, the application of the group $1 \mathrm{mGluR}$ antagonist MCPG did not reduce the DSI of mIPSCs in Purkinje cells (Glitsch, PhD thesis, 1998). In contrast, AM-251 led to a strong overshooting of spike rate in current-clamped interneurons after that saturating concentrations of the agonist WIN55, 212-2 decreased firing (Fig.6.20) and this would, indeed, be explicable with a basal activation of CB1Rs in control.

These partially contradictory results indicate that ambient glutamate might not be sufficient to trigger the cannabinoid machinery in the cerebellum. Alternatively, given the predominant localization of group $1 \mathrm{mGluRs}$ at the perifery of excitatory synapses, the passive diffusion of cannabinoids to GABAergic presynaptic terminals may not prove sufficient to activate CB1Rs under resting conditions.

The use of cannabinoid transporter blockers like AM-404 might provide important information; it actually did in the hippocampus, where Wilson and Nicoll, 2001, managed to uncover an inhibition of GABAergic transmission by ambient cannabinoids in this way.

\subsection{Controlling presynaptic spike rate: a novel form of plasticity}

The data of this dissertation describe a novel mechanism by which synaptic activity can be retrogradely modulated: postsynaptically produced cannabinoids inhibit the generation of action potentials in nearby presynaptic cells. Thus, they confirm a report published by other authors during the writing of this thesis (Kreitzer et al., 2002) and actually expand it by revealing an earlier excitatory effect of Purkinje cell depolarizations on the spike rate of basket/stellate cells. Importantly, these findings also provide an explanation to the TTX-dependent propagation of DSI in unstimulated Purkinje cells, which was reported by Vincent and Marty, 1993 (see section 2.1.4). 


\subsubsection{The "early", excitatory effect}

The faster effect on firing was excitatory; it typically developed with a delay of 100-200 ms after the onset of the DSI protocol (Fig.6.22A). In contrast to the later inhibitory phenomenon, it is still unclear what mediates it. Which hypotheses could be envisaged?

First of all, it was important to show that the effect was not dependent on the accumulation of extracellular cesium issueing from the postsynaptic compartment during the DSI protocols (Fig.6.22Ba). Possible artifacts deriving from the recording conditions could be, thus, excluded, although a role for a transient change in the ionic composition of the extracellular medium must still be considered. Dendritic depolarizations can indeed produce transient depletions of extracellular calcium (see for a modeling study: Egelman and Montague, 1998) after which, as a consequence, very brief periods of synaptic depressions can follow (like in the Calix of Held: Borst and Sakmann, 1999). Moreover, and importantly for this discussion, extracellular solutions devoid of calcium are known to induce epileptic-like activity and general increases in neuronal membrane excitability (Konnerth et al., 1986; Bouskila and Dudek, 1993; Li and Hatton, 1996 and, importantly, in cerebellar interneurons: Dugue', DEA thesis, 2002). Although DSI itself cannot be explained by this mechanism, at this stage it is nonetheless still consistent to imagine that transient calcium depletions due to strong Purkinje cell depolarizations could underly the early increase in spike rate.

Are CB1Rs involved? The onset of this phenomenon is actually compatible with a G-protein mediated mechanism. However, only few $(n=5)$ experiments were performed to approach this question and no straightforward results were obtained due to the great variability of this phenomenon in single experiments. (but see Fig.6.18). Further trials will be performed and, conjointly, CB1R K.O. mice will be used in the near future with this goal.

Finally, particularly interesting is the hypothesis that anandamide, but not CB1Rs, may be involved; anandamide can directly block several voltage-dependent conductances in a G-protein independent way: inhibitory effects have been described on the Shaker related Kv1.2 potassium channel (Poling et al., 1996; this subunit is present and functional in basket cell terminals: Laube et al., 1996; Southan and Robertson, 2000), on T-type calcium channels (Chemin et al., 2001) and on the background $\mathrm{K}^{+}$channel TASK-1 (Maingret et al., 2001).

It will be important in future experiments to try and identify the small voltage-dependent inward current, which developed in interneurons during the postsynaptic pulse (Fig.6.22Bb); even a small change of membrane conductance can infact give rise to strong changes in firing in cells 
with very high input resistance, as our interneurons are.

\subsubsection{The "late", inhibitory effect}

The observation of a retrograde inhibition of firing amplifies the potential of cannabinoid signalling in the cerebellum: the modulation of afferent firing, infact, dramatically extends the domain over which a single Purkinje cell can modulate the activity of other Purkinje cells and, so, the cerebellar output.

The phenomenon seemed to be dependent on the spatial range of diffusion of endocannabinoids in the extracellular environment: the decline of inhibition showed indeed a similar dependence on intrasomatic distance both for connected and not connected pairs (Fig.6.10). The concentration and the rate of activity of the specific cannabinoid transporter on neuronal and glial membranes (Beltramo et al., 1997) are likely to be limiting factors for the setting of the space constant; hence, it would be interesting to see whether the spatial extension of inhibition significantly changes at physiological temperatures, where transporters should work at much higher efficiency. In this regard, Kreitzer et al., 2002, have actually shown that, in contrast with DSI, DSE spreading disappeared almost completely at high temperature, thus attesting an increased transporter activity and a more limited diffusion of endocannabinoids in these nearly physiological conditions; furthermore, this observation raises important questions about the relative localization of the cannabinoid synthesis machinery with respect to the excitatory/inhibitory synaptic inputs onto Purkinje cells and, thus, about the synapse specificity of endocannabinoid actions.

There are relevant differences between the work of Kreitzer et al. and my data: these authors generally set the threshold intrasomatic distance, within which the effect was detected, at $100 \mu \mathrm{m}$ but did not provide any more detailed information about the maximal inhibition or its quantitative course. Here, I attest that Purkinje cell depolarizations could silence presynaptic interneurons almost completely, provided that the 2 cells were sufficiently close $(91.22 \%$ inhibition at less than $25 \mu \mathrm{m}$ ) and measured a precise rate of decline.

Furthermore and in contrast with Kreitzer et al., my experiments show that unconnected interneurons experienced significantly less inhibition than connected interneurons; the difference in the age of the animals used (14 to 21 days old instead of our 11 to 15 days old) could account for the discrepancies in this last point. On average, Kreitzer et al. have worked with more 
developed Purkinje cells spreading over much wider regions of the ML: this could explain the longer distance over which the effect was detected, and could have reduced the effective path for cannabinoids to cover in the extracellular space in order to activate CB1Rs, thus making the existence of closely juxtapposed membrane irrilevant for the expression of the phenomenon.

CB1R activation is, as for the DSI of synaptic currents, a necessary and sufficient condition for spike inhibition. The pharmacological block of the receptors totally hindered any change in firing due to the induction of DSI and, conversely, WIN 55,212-2 significantly reduced the firing of interneurons, even though with a smaller effectiveness than endogenous cannabinoids (see the difference in maximal inhibition by DSI for small distances, compared with the effect of WIN). Furthermore, the large majority (7 out of 9) of these experiments have been performed with bicuculline in the bath, besides the usual cocktail of glutamatergic receptor antagonists; this shows that the inhibitory effect did not depend on the concomitant modulation of synaptic activity, which cannabinoids exerted.

Kreitzer et al. described 2 other observations in agreement with my findings: AM-251 reversed and overshot the effect of WIN 55,212-2 on the firing of interneurons and, secondly, they described the same outward current developing during CB1R activation as I have in the same conditions but also following the endogenous production of cannabinoids (Fig.6.22).

The former observation would suggest a basal activation of the cannabinoid system in control, even though I have in detail outlined in section 7.5.1 that the data concerning CB1Rs and synaptic transmission do not support this. An intriguing and speculative explanation for this discrepancy could be found, if the facilitatory effect on firing were actually originating by cannabinoids acting independently from CB1 receptors. In this scheme the facilitatory effect, continuously triggered by the saturating concentration of WIN in the bath, would tonically oppose the inhibitory one from CB1Rs and could be uncovered when these were pharmacologically eliminated.

Concerning the latter point (the outward current), the 2 authors proposed that CB1Rs upregulated a small inwardly rectifying potassium conductance. The pharmacological profile of the current was the following: block by intracellular cesium and TEA, by extracellular barium and lack of sensitivity to 4-AP, an unspecific blocker of Ia currents. My data are consistent with this scheme, although also a role for a down-regulation of Ih could be envisaged. A concomitant modulation of Ih and of a GIRK-like channel was already observed in hippocampal interneurons (Svoboda and Lupica, 1998) after activation of $\mu$-opioid receptors. Interestingly, these receptors 
activate the same pertussis toxin-sensitive G protein as CB1Rs. The conjoint regulation of these conductances would fully explain the dramatic effect on firing exerted by CB1Rs.

Finally, once more it must not be forgotten that the conductance changes recorded at the somatic level are likely to take place also down the axon; this would imply the possibility for conduction failures to originate also, for example, at axonal bifurcation points. In this case, transmission failures would be detected by the postsynaptic sensor but could not be associated with spike failures at the soma of interneurons. This is an experimentally much harder hypothesis to approach; calcium imaging experiments in CNS neurons never reported the occurrence of conduction failures (see my imaging experiments and also Forti et al., 2000; Cox, et al., 2000 and Koester and Sakmann, 2001). The invasive characteristics of the technique employed for these experiments, nonetheless, still leave the question open for further verification.

\subsubsection{A comparison with the hippocampus}

Alger et al., 1996, proposed that also hippocampal DSI could be related to signal propagation along axons. The starting point was the lack in any change in PPR, which appeared inconsistent with a direct effect of DSI on the release machinery.

The recent works of Wilson and Nicoll, 2001 and of Wilson et al., 2001 invalidated the propagation failure thesis. They advanced the following arguments:

- the PPR actually increased during DSI, consistently with a decrease of presynaptic release probability;

- DSI actually spread between CA1 pyramidal cells. The depression of sIPSCs could be detected also in cells which had not been depolarized; but, in contrast to the cerebellum, this was limited to a very small region surrounding the stimulated cell: it faded out at distances greater than $20 \mu \mathrm{m}$, which was within the spatial limits of a passive diffusive process;

- again in contrast to cerebellar DSI, this spreading was not correlated with the amount of common input CA1 cells had.

These elements suggest that the spread of hippocampal DSI was accomplished through the passive diffusion of endogenous cannabinoids from their site of production; this also implies that no modification in the effectiveness with which action potentials were generated or did propagate occured. 


\subsection{Cannabinoids and gap junctions}

GABAergic interneurons in the cerebellar molecular layer form a widespread network of chemically and electrically connected cells. This is what my experiments suggest; moreover, they confirm what was found morphologically in adult cats and rats (Sotelo and Llinas, 1972) and physiologically in guinea pigs (Mann-Metzer and Yarom, 1999) and extend to the cerebellum what is a generalized property of GABAergic interneurons in the CNS. Inhibitory interneurons are connected by chemical and electrical synapses in many central areas, like in the neocortex (Galarreta and Hestrin, 1999 and Gibson et al., 1999; Tamas et al., 2000) and in the hippocampus (see for example in the latest literature: Fukuda and Kosaka, 2000 and Meyer et al., 2002), where this subject has been studied most extensively. Networks of interneurons can generate synchronized spiking under many different pharmacological conditions (Michelson and Wong, 1994 and Whittington et al., 1995 and Traub et al., 2001 for a modeling study); this can, in principle, be originated both by chemical or electrical synapses.

My data do not support an extensive synchronization of firing between interneurons in basal conditions; only in very few cases, a temporal correlation between full spikes and spikelets could be observed (like for the experiment in Fig.6.14). Typically, synchronized activity could be seen only after pharmacological treatments that substantially increased the firing rate, like aspecific $\mathrm{K}^{+}$channel blockers (with barium and TEA; data not shown); depletion of extracellular calcium was also used in another report to achieve the same effect (Dugue', DEA thesis, 2002). Moreover, spikelets were observed without distinction in basket cells as well as in low stellate cells. Both these observations are in contrast with what reported by Mann-Metzer and Yarom, 1999: these authors noticed a high level of firing synchronization in paired recordings in control conditions and a coupling limited only to stellate cells in the higher portion of the molecular layer.

A low basal firing frequency, a weak coupling conductance between interneurons and/or the lack of a spontaneous excitatory drive (parallel and climbing fibers are almost silent in the slice preparation) could explain the lack of synchronization in my experiments. An increased spike rate might be reached by performing the experiments at near physiological temperatures while an evaluation of the coupling conductance will be performed in future paired recordings between interneurons.

Purkine cell depolarizations are able to modulate biphasically the firing of a large population of interneurons; this could be seen both from the effects on the firing of the recorded cells but 
also through the effect on spikelets (Fig.6.17). It is important to stress that electrical coupling can, in principle, amplify both the absolute magnitude and the area over which cannabinoids exert a significant effect; an interneuron out of the area covered by diffusing cannabinoids might infact still feel their indirect action through other electrically connected cells, placed closer to the source. In this way, the activation of CB1Rs on a small sample of cells might disrupt the synchronized output of a whole network extending over several hundred micrometers.

Finally, gap junctions can be directly modulated by neurotransmitters: muscarinic, dopamine and NMDA receptors have been reported to change the degree of electrical coupling between neurons (Rorig et al., 1995; Pereda and Faber, 1996; Velazquez et al., 1997), in particular through cAMP-mediated phosphorylation of particular connexins (Lasater, 1987; De Vries and Schwartz, 1989). Also this possibility will have to be seriously considered to assess completely how CB1Rs can affect the functionality of interneuron networks in the cerebellum. 


\section{Bibliography}

[1] B.E. Abe, H. Sugihara, H. Nawa, R. Shigemoto, N. Mizuno, and S. Nakanishi. Molecular characterization of a novel metabotropic glutamate receptor mGluR5 coupled to inositol phosphate/Ca2+ signal transduction. J. Biol. Chem., 267: 13361-8, 1992.

[2] B.E. Alger, T.A. Pitler, J.J. Wagner, L.A. Martin, W. Morishita, S.A. Kirov, and R.A. Lenz. Retrograde signalling in Depolarization-induced Suppression of Inhibition in rat hippocampal CA1 cells. J. Physiol., 496: 197-209, 1996.

[3] A. Al-Hayani, K.N. Wease, R.A. Ross, R.G. Pertwee and S.N. Davies. The endogenous cannabinoid anandamide activates vanilloid receptors in the rat hippocampal slice. Neuropharmacology. 41: 1000-5, 2001.

[4] J. Altman and S.A. Bayer. Development of the cerebellar system: in relation to its evolution, structure and functions. CRC Press, Boca Raton, FL, USA, 1997.

[5] A. Ameri, A. Wilhelm, and T. Simmet. Effects of the endogenous cannabinoid, anandamide, on neuronal activity in rat hippocampal slices. Br. J. Pharmacol., 126: 1831-9, 1999.

[6] I. Aramori and S. Nakanishi. Signal transduction and pharmacological characteristics of a metabotropic glutamate receptor, mGluR1, in transfected CHO cells. Neuron, 8: 757-65, 1992.

[7] N. Auclair, S. Otani, P. Soubrie, and F. Crepel. Cannabinoids modulate synaptic strength and plasticity at glutamatergic synapses of rat prefrontal cortex pyramidal neurons. $J$. Neurophysiol., 83: 3287-93, 2000.

[8] G.J. Augustine. Regulation of transmitter release at the squid giant synapse by presynaptic delayed rectifier potassium current. J. Physiol., 431: 343-64, 1990. 
[9] G.J. Augustine and M.P. Charlton. Calcium dependence of presynaptic calcium current and post-synaptic response at the squid giant synapse. J. Physiol., 381: 619-40, 1986.

[10] A.M. Batchelor and J. Garthwaite. Novel synaptic potentials in cerebellar Purkinje cells: probable mediation by metabotropic glutamate receptors. Neuropharmacology, 32: 11$20,1993$.

[11] A.M. Batchelor and J. Garthwaite. Frequency detection and temporally dispersed synaptic signal association through a metabotropic glutamate receptor pathway. Nature, 385: 74-7, 1997.

[12] A. Baude, Z. Nusser, J.D. Roberts, E. Mulvihill, R.A. McIlhinney, and P. Somogyi. The metabotropic glutamate receptor (mGluR1 alpha) is concentrated at perisynaptic membrane of neuronal subpopulations as detected by immunogold reaction. Neuron, 11: 771-87, 1993.

[13] V. Beaumont and R.S. Zucker. Enhancement of synaptic transmission by cyclic AMP modulation of presynaptic Ih channels. Nat. Neurosci., 3: 133-41, 2000.

[14] J.M. Bekkers and C.F. Stevens. Presynaptic mechanism for Long-Term Potentiation in the hippocampus. Nature, 346: 724-9, 1990.

[15] M. Beierlein, J.R. Gibson, and B.W. Connors. A network of electrically coupled interneurons drives synchronized inhibition in neocortex. Nature Neurosci., 3: 904-10, 2000.

[16] M. Beltramo and D. Piomelli. Carrier-mediated transport and enzymatic hydrolysis of the endogenous cannabinoid 2-arachidonylglycerol. Neuroreport, 11: 1231-5, 2000.

[17] M. Beltramo, N. Stella, A. Calignano, S.Y. Lin, A. Makriyannis, and D. Piomelli. Functional role of high-affinity anandamide transport, as revealed by selective inhibition. Science, 277: 1094-7, 1997.

[18] T.V. Bliss and G.L. Collingridge. A synaptic model of memory: Long-Term Potentiation in the hippocampus. Nature, 361: 31-9, 1993.

[19] J.H. Bollmann, B. Sakmann, and J.G. Borst. Calcium sensitivity of glutamate release in a Calyx-type terminal. Science, 289: 953-7, 2000. 
[20] J.G. Borst and B. Sakmann. Depletion of calcium in the synaptic cleft of a Calyx-type synapse in the rat brainstem. J. Physiol., 521: 123-33, 1999.

[21] M. Bouaboula, C. Poinot-Chazel, B. Bourrie, X. Canat, B. Calandra, M. RinaldiCarmona, G. Le Fur, and P. Casellas. Activation of Mitogen-Activated Protein kinases by stimulation of the central cannabinoid receptor CB1. Biochem. J., 312: 637-41, 1995.

[22] Y. Bouskila and F.E. Dudek. Neuronal synchronization without calcium-dependent synaptic transmission in the hypothalamus. Proc. Natl. Acad. Sci. U.S.A., 90: 3207-10, 1993.

[23] C.S. Breivogel, G. Griffin, V. Di Marzo, and B.R. Martin. Evidence for a new G proteincoupled cannabinoid receptor in mouse brain. Mol Pharmacol, 60: 155-63, 2001.

[24] H. Cadas, E. Di Tomaso, and D. Piomelli. Occurrence and biosynthesis of endogenous cannabinoid precursor, n- arachidonoyl phosphatidylethanolamine, in rat brain. J. Neurosci., 17: 1226-42, 1997.

[25] H. Cadas, M. Gaillet, S. Beltramo, L. Venance, and D. Piomelli. Biosynthesis of an endogenous cannabinoid precursor in neurons and its control by calcium and cAMP. $J$. Neurosci., 16: 3934-42, 1996.

[26] O. Caillard, H. Moreno, B. Schwaller, I. Llano, M.R. Celio, and A. Marty. Role of the calcium-binding protein parvalbumin in short-term synaptic plasticity. Proc. Natl. Acad. Sci. U.S.A., 97: 13372-7, 2000.

[27] A. Calignano, G. La Rana, A. Beltramo, M. Makriyannis, and D. Piomelli. Potentiation of anandamide hypotension by the transport inhibitor, AM404. Eur. J. Pharmacol., 337: R1-2, 1997.

[28] J.C. Callaway, N. Lasser-Ross, and W.N. Ross. IPSPs strongly inhibit climbing fiberactivated $[\mathrm{Ca} 2+] \mathrm{i}$ increases in the dendrites of cerebellar Purkinje neurons. J. Neurosci., 15: 2777-87, 1995.

[29] A.G. Carter and W.G. Regehr. Prolonged synaptic currents and glutamate spillover at the parallel fiber to stellate cell synapse. J. Neurosci., 20: 4423-34, 2000.

[30] M.J. Caterina and D. Julius. The vanilloid receptor: a molecular gateway to the pain pathway. Annu Rev Neurosci., 24: 487-517, 2001. 
[31] M.P. Caulfield and D.A. Brown. Cannabinoid receptor agonists inhibit Ca current in NG108-15 neuroblastoma cells via a pertussis toxin-sensitive mechanism. Pflugers Arch. 420: 486-92, 1992.

[32] C.Y. Chan, J. Hounsgaard, and J. Midtgaard. Excitatory synaptic responses in turtle cerebellar Purkinje cells. J. Physiol., 409: 143-56, 1989.

[33] P.K. Chan and W.H. Yung. Occlusion of the presynaptic action of cannabinoids in rat substantia nigra pars reticulata by cadmium. Neurosci Lett, 249: 57-60, 1998.

[34] J. Chemin, A. Monteil, E. Perez-Reyes, J. Nargeot, and P. Lory. Direct inhibition of Ttype calcium channels by the endogenous cannabinoid anandamide. Embo J., 20: 703340, 2001.

[35] C.J. Coates and A.G. Bulloch. Synaptic plasticity in the molluscan peripheral nervous system: physiology and role for peptides. J. Neurosci., 5: 2677-84, 1985.

[36] S. Coco, G. Raposo, S. Martinez, J.J. Fontaine, S. Takamori, A. Zahraoui, R. Jahn, M. Matteoli, D. Louvard, and T. Galli. Subcellular localization of tetanus neurotoxininsensitive vesicle- associated membrane protein (VAMP)/VAMP7 in neuronal cells: evidence for a novel membrane compartment. J. Neurosci., 19: 9803-12, 1999.

[37] W. A. Coetzee, Y. Amarillo, J. Chiu, A. Chow, D. Lau, T. McCormack, H. Moreno, M. S. Nadal, A. Ozaita, D. Pountney, M. Saganich, E. Vega-Saenz de Miera, and B. Rudy. Molecular diversity of K+ channels. Ann. N.Y. Acad. Sci., 868: 233-85, 1999.

[38] C. L. Cox, W. Denk, D. W. Tank, and K. Svoboda. Action potentials reliably invade axonal arbors of rat neocortical neurons. Proc. Natl. Acad. Sci. U.S.A., 97: 9724-8, 2000.

[39] B.F. Cravatt, K. Demarest, M.P. Patricelli, M.H. Bracey, D.K. Giang, B.R. Martin, and A.H. Lichtman. Supersensitivity to anandamide and enhanced endogenous cannabinoid signaling in mice lacking Fatty Acid Amide Hydrolase. Proc. Natl. Acad. Sci. U.S.A., 98: 9371-6, 2001.

[40] B.F. Cravatt, D.K. Giang, S.P. Mayfield, D.L. Boger, R.A. Lerner, and N.B. Gilula. Molecular characterization of an enzyme that degrades neuromodulatory fatty-acid amides. Nature, 384: 83-7, 1996. 
[41] K.P. Currie and A.P. Fox. Comparison of N- and P/Q-type voltage-gated calcium channel current inhibition. J. Neurosci., 17: 4570-9, 1997.

[42] H. Daniel and F. Crepel. Control of $\mathrm{Ca}(2+)$ influx by cannabinoid and metabotropic glutamate receptors in rat cerebellar cortex requires $\mathrm{K}(+)$ channels. J. Physiol., 537: 793-800, 2001.

[43] J. deRooij, F.J. Zwartkruis, M.H. Verheijen, R.H. Cool, S.M. Nijman, A. Wittinghofer, and J.L. Bos. EPAC is a RAP1 guanine-nucleotide-exchange factor directly activated by cyclic AMP. Nature, 396: 474-7, 1998.

[44] S.A. Deadwyler, R.E. Hampson, B.A. Bennett, T.A. Edwards, J. Mu, M.A. Pacheco, S.J. Ward, and S.R. Childers. Cannabinoids modulate potassium current in cultured hippocampal neurons. Receptors Channels, 1: 121-34, 1993.

[45] S.A. Deadwyler, R.E. Hampson, J. Mu, A. Whyte, and S. Childers. Cannabinoids modulate voltage sensitive potassium A-current in hippocampal neurons via a cAMPdependent process. J. Pharmacol. Exp. Ther., 273: 734-43, 1995.

[46] D. Debanne, N.C. Guerineau, B.H. Gahwiler, and S.M. Thompson. Action-potential propagation gated by an axonal I(A)-like $\mathrm{K}+$ conductance in hippocampus. Nature, 389: 286-9, 1997.

[47] K. Delaney, D.W. Tank, and R.S. Zucker. Presynaptic calcium and serotonin-mediated enhancement of transmitter release at crayfish neuromuscular junction. J. Neurosci., 11: 2631-43, 1991.

[48] W.A. Devane, F.A. Dysarz, M.R. Johnson, L.S. Melvin, and A.C. Howlett. Determination and characterization of a cannabinoid receptor in rat brain. Mol. Pharmacol., 34: 605-13, 1988.

[49] W.A. Devane, L. Hanus, A. Breuer, R.G. Pertwee, L.A. Stevenson, G. Griffin, D. Gibson, A. Mandelbaum, A. Etinger, and R. Mechoulam. Isolation and structure of a brain constituent that binds to the cannabinoid receptor. Science, 258: 1946-9, 1992.

[50] S.H. DeVries and E.A. Schwartz. Modulation of an electrical synapse between solitary pairs of catfish horizontal cells by dopamine and second messengers. J. Physiol., 414: $351-75,1989$. 
[51] V. Di Marzo, A. Fontana, H. Cadas, S. Schinelli, G. Cimino, J.C. Schwartz, and D. Piomelli. Formation and inactivation of endogenous cannabinoid anandamide in central neurons. Nature, 372: 686-91, 1994.

[52] V. Di Marzo, D. Melck, T. Bisogno, and L. De Petrocellis. Endocannabinoids: endogenous cannabinoid receptor ligands with neuromodulatory action. Trends Neurosci., 21: 521-8, 1998.

[53] S. Dieudonne and A. Dumoulin. Serotonin-driven long-range inhibitory connections in the cerebellar cortex. J. Neurosci., 20: 1837-48, 2000.

[54] T.P. Dinh, D. Carpenter, F.M. Leslie, T.F. Freund, I. Katona, S.L. Sensi, S. Kathuria, and D. Piomelli. Brain monoglyceride lipase participating in endocannabinoid inactivation. Proc. Natl. Acad. Sci. U.S.A., 99: 10819-24, 2002.

[55] F.A. Dodge and R. Rahamimoff. Co-operative action a calcium ions in transmitter release at the neuromuscular junction. J. Physiol., 193: 419-32, 1967.

[56] C.T. Drake, G.W. Terman, M.L. Simmons, T.A. Milner, D.D. Kunkel, P.A. Schwartzkroin, and C. Chavkin. Dynorphin opioids present in dentate granule cells may function as retrograde inhibitory neurotransmitters. J. Neurosci., 14: 3736-50, 1994.

[57] G. Dugué. Electrical coupling between cerebellar interneurons. DEA thesis, Université Paris VII, 2002.

[58] J.C. Eccles, R. Llinas, and K. Sasaki. The inhibitory interneurones within the cerebellar cortex. Exp. Brain. Res., 1: 1-16, 1966.

[59] D.M. Egelman and P.R. Montague. Computational properties of peri-dendritic calcium fluctuations. J. Neurosci., 18: 8580-9, 1998.

[60] M. Egertova and M.R. Elphick. Localisation of cannabinoid receptors in the rat brain using antibodies to the intracellular C-terminal tail of cb. J. Comp. Neurol., 422: 159-71, 2000 .

[61] M. Egertova, D.K. Giang, B.F. Cravatt, and M.R. Elphick. A new perspective on cannabinoid signalling: complementary localization of Fatty Acid Amide Hydrolase and the CB1 receptor in rat brain. Proc R Soc Lond B Biol Sci, 265: 2081-5, 1998. 
[62] D.S. Faber and H. Korn. Applicability of the coefficient of variation method for analyzing synaptic plasticity. Biophys. J., 496: 1288-1294, 1991.

[63] L. Fierro and I. Llano. High endogenous calcium buffering in Purkinje cells from rat cerebellar slices. J. Physiol., 496: 617-25, 1996.

[64] R.M. Fitzsimonds and M.M. Poo. Retrograde signaling in the development and modification of synapses. Physiol Rev, 78(1): 143-70, 1998.

[65] E.M. Fykse, C. Li and T.C. Sudhof. Phosphorylation of rabphilin-3A by Ca2+/calmodulin- and cAMP-dependent protein kinases in vitro. J. Neurosci., 15: 2385$95,1995$.

[66] L. Forti, C. Pouzat, and I. Llano. Action potential-evoked $\mathrm{Ca} 2+$ signals and calcium channels in axons of developing rat cerebellar interneurones. J. Physiol., 527: 33-48, 2000.

[67] T. Fukuda and T. Kosaka. Gap junctions linking the dendritic network of GABAergic interneurons in the hippocampus. J. Neurosci., 20(4): 1519-28, 2000.

[68] M. Galarreta and S. Hestrin. A network of fast-spiking cells in the neocortex connected by electrical synapses. Nature, 402: 72-5, 1999.

[69] M. Galarreta and S. Hestrin. Electrical synapses between GABA-releasing interneurons. Nat. Rev. Neurosci., 2: 425-33, 2001.

[70] S. Galiegue, S. Mary, J. Marchand, D. Dussossoy, D. Carriere, P. Carayon, M. Bouaboula, D. Shire, G. Le Fur, and P. Casellas. Expression of central and peripheral cannabinoid receptors in human immune tissues and leukocyte subpopulations. Eur. J. Biochem., 232: 54-61, 1995.

[71] T. Galli, A. Zahraoui, V.V. Vaidyanathan, G. Raposo, J.M. Tian, M. Karin, H. Niemann, and D. Louvard. A novel tetanus neurotoxin-insensitive vesicle-associated membrane protein in SNARE complexes of the apical plasma membrane of epithelial cells. Mol. Biol. Cell., 9: 1437-48, 1998.

[72] J.R. Geiger and P. Jonas. Dynamic control of presynaptic $\mathrm{Ca}(2+)$ inflow by fastinactivating $\mathrm{K}(+)$ channels in hippocampal mossy fiber boutons. Neuron, 28: 927-39, 2000. 
[73] G. Gerdeman and D.M. Lovinger. CB1 cannabinoid receptor inhibits synaptic release of glutamate in rat dorsolateral striatum. J. Neurophysiol., 85: 468-71, 2001.

[74] G.L. Gerdeman, J. Ronesi, and D.M. Lovinger. Postsynaptic endocannabinoid release is critical to long-term depression in the striatum. Nat. Neurosci., 5: 446-51, 2002.

[75] R.W. Gereau IV and P.J. Conn. Roles of specific metabotropic glutamate receptor subtypes in regulation of hippocampal ca1 pyramidal cell excitability. J. Neurophysiol., 74: 122-9, 1995.

[76] J.R. Gibson, M. Beierlein, and B.W. Connors. Two networks of electrically coupled inhibitory neurons in neocortex. Nature, 402: 75-9, 1999.

[77] A. Giuffrida, L. H. Parsons, T.M. Kerr, F. Rodriguez de Fonseca, M. Navarro, and D. Piomelli. Dopamine activation of endogenous cannabinoid signaling in dorsal striatum. Nat. Neurosci., 2: 358-63, 1999.

[78] M. Glitsch. Modulation inhibitorischer synaptischer transmission und identifizierung eines retrograden botenstoffes in kleinhirnschnitten der ratte. PhD thesis, Georg-AugustUniversitaet Goettingen, 1998.

[79] M. Glitsch, I. Llano, and A. Marty. Glutamate as a candidate retrograde messenger at interneurone-Purkinje cell synapses of rat cerebellum. J. Physiol., 497: 531-7, 1996.

[80] M. Glitsch and A. Marty. Presynaptic effects of NMDA in cerebellar Purkinje cells and interneurons. J. Neurosci., 19: 511-9, 1999.

[81] M.D. Glitsch and J.J. Jack. Evidence that glutamate acting on presynaptic type-II metabotropic glutamate receptors alone does not fully account for the phenomenon of Depolarisation-induced Suppression of Inhibition in cerebellar Purkinje cells. Pflugers Arch., 442: 404-8, 2001.

[82] N. Hajos, I. Katona, S.S. Naiem, K. MacKie, C. Ledent, I. Mody, and T.F. Freund. Cannabinoids inhibit hippocampal GABAergic transmission and network oscillations. Eur J. Neurosci., 12: 3239-49, 2000.

[83] N. Hajos, C. Ledent, and T.F. Freund. Novel cannabinoid-sensitive receptor mediates inhibition of glutamatergic synaptic transmission in the hippocampus. Neuroscience, 106: $1-4,2001$. 
[84] R.E. Hampson, G.J. Evans, J. Mu, S.Y. Zhuang, V.C. King, S.R. Childers, and S.A. Deadwyler. Role of cyclic AMP dependent protein kinase in cannabinoid receptor modulation of potassium A-current in cultured rat hippocampal neurons. Life Sci., 56: 2081-8, 1995.

[85] M. Hausser and B.A. Clark. Tonic synaptic inhibition modulates neuronal output pattern and spatiotemporal synaptic integration. Neuron, 19: 665-78, 1997.

[86] P.G. Haydon. Glia: listening and talking to the synapse. Nat. Rev. Neurosci., 2: 185-93, 2001.

[87] F. Helmchen, K. Imoto, and B. Sakmann. Ca2+ buffering and action potential-evoked Ca2+ signaling in dendrites of pyramidal neurons. Biophys. J., 70: 1069-81, 1996.

[88] D.J. Henry and C. Chavkin. Activation of inwardly rectifying potassium channels (GIRK1) by co- expressed rat brain cannabinoid receptors in Xenopus oocytes. Neurosci. Lett., 186: 91-4, 1995.

[89] M. Herkenham, A.B. Lynn, M.R. Johnson, L.S. Melvin, B.R. de Costa, and K.C. Rice. Characterization and localization of cannabinoid receptors in rat brain: a quantitative in vitro autoradiographic study. J. Neurosci., 11: 563-83, 1991.

[90] M. Herkenham, A.B. Lynn, M.D. Little, M.R. Johnson, L.S. Melvin, B.R. de Costa, and K.C. Rice. Cannabinoid receptor localization in brain. Proc. Natl. Acad. Sci. U.S.A., 87: 1932-6, 1990.

[91] S. Herlitze, D.E. Garcia, K. Mackie, B. Hille, T. Scheuer, and W.A. Catterall. Modulation of Ca2+ channels by G-protein beta gamma subunits. Nature, 380: 258-62, 1996.

[92] B.Y. Ho, Y. Uezono, S. Takada, I. Takase, and F. Izumi. Coupling of the expressed cannabinoid $\mathrm{CB} 1$ and $\mathrm{CB} 2$ receptors to phospholipase $\mathrm{C}$ and $\mathrm{G}$ protein-coupled inwardly rectifying K+ channels. Receptors Channels, 6: 363-74, 1999.

[93] A.F. Hoffman and C.R. Lupica. Mechanisms of cannabinoid inhibition of GABA(a) synaptic transmission in the hippocampus. J. Neurosci., 20: 2470-9, 2000.

[94] M. Hosaka, R.E. Hammer, and T.C. Sudhof. A phospho-switch controls the dynamic association of Synapsins with synaptic vesicles. Neuron, 24: 377-87, 1999. 
[95] A.C. Howlett and R.M. Fleming. Cannabinoid inhibition of Adenylate Cyclase. Pharmacology of the response in neuroblastoma cell membranes. Mol. Pharmacol., 26: 532-8, 1984.

[96] C.C. Huang, S.W. Lo, and K.S. Hsu. Presynaptic mechanisms underlying cannabinoid inhibition of excitatory synaptic transmission in rat striatal neurons. J. Physiol., 532: $731-48,2001$.

[97] M. Iizuka, I. Tsunenari, Y. Momota, I. Akiba, and T. Kono. Localization of a G-proteincoupled inwardly rectifying $\mathrm{K}+$ channel, CIR, in the rat brain. Neuroscience, 77: 1-13, 1997.

[98] S.R. Ikeda. Voltage-dependent modulation of N-type calcium channels by G-protein beta gamma subunits. Nature, 380: 255-8, 1996.

[99] M. Ito. Cerebellar long-term depression: characterization, signal transduction, and functional roles. Physiol. Rev., 81: 1143-95, 2001.

[100] S. Iwasaki, A. Momiyama, O.D. Uchitel, and T. Takahashi. Developmental changes in calcium channel types mediating central synaptic transmission. J. Neurosci., 20: 59-65, 2000 .

[101] M.B. Jackson, A. Konnerth, and G.J. Augustine. Action potential broadening and frequency-dependent facilitation of calcium signals in pituitary nerve terminals. Proc. Natl. Acad. Sci. U.S.A., 88: 380-4, 1991.

[102] E.H. Jaffe, A. Marty, A. Schulte and R.H. Chow. Extrasynaptic vesicular transmitter release from the somata of substantia nigra neurons in rat midbrain slices. J. Neurosci., 18: 3548-59, 1998.

[103] E.A. Jennings, C.W. Vaughan, and M.J. Christie. Cannabinoid actions on rat superficial medullary dorsal horn neurons in vitro. J. Physiol., 534: 805-12, 2001.

[104] M. Kano, U. Rexhausen, J. Dreessen, and A. Konnerth. Synaptic excitation produces a long-lasting rebound potentiation of inhibitory synaptic signals in cerebellar Purkinje cells. Nature, 356: 601-4, 1992. 
[105] K. Kato, G.D. Clark, N.G. Bazan, and C.F. Zorumski. Platelet-Activating Factor as a potential retrograde messenger in CA hippocampal long-term potentiation. Nature, 367: 175-9, 1994.

[106] I. Katona, E.A. Rancz, L. Acsady, C. Ledent, K. Mackie, N. Hajos, and T.F. Freund. Distribution of CB1 cannabinoid receptors in the amygdala and their role in the control of GABAergic transmission. J. Neurosci., 21: 9506-18, 2001.

[107] I. Katona, B. Sperlagh, A. Sik, A. Kafalvi, E.S. Vizi, K. Mackie, and T.F. Freund. Presynaptically located CB1 cannabinoid receptors regulate GABA release from axon terminals of specific hippocampal interneurons. J. Neurosci., 19: 4544-58, 1999.

[108] B. Katz and R. Miledi. The timing of calcium action during neuromuscular transmission. J. Physiol., 189: 535-44, 1967.

[109] K. Kaupmann, K. Huggel, J. Heid, P.J. Flor, S. Bischoff, S.J. Mickel, G. McMaster, C. Angst, H. Bittiger, W. Froestl, and B. Bettler. Expression cloning of GABA(b) receptors uncovers similarity to metabotropic glutamate receptors. Nature, 386: 239-46, 1997.

[110] H. Kawasaki, G.M. Springett, N. Mochizuki, S. Toki, M. Nakaya, M. Matsuda, D.E. Housman, and A.M. Graybiel. A family of cAMP-binding proteins that directly activate RAP1. Science, 282(5397): 2275-9, 1998.

[111] J. Kim and B.E. Alger. Random response fluctuations lead to spurious paired-pulse facilitation. J. Neurosci., 21: 9608-18, 2001.

[112] M.T. Kirby, R.E. Hampson, and S.A. Deadwyler. Cannabinoid receptor activation in ca1 pyramidal cells in adult rat hippocampus. Brain Res., 863: 120-31, 2000.

[113] M. Klein, J. Camardo, and E.R. Kandel. Serotonin modulates a specific potassium current in the sensory neurons that show presynaptic facilitation in Aplysia. Proc. Natl. Acad. Sci. U.S.A., 79: 5713-7, 1982.

[114] H.J. Koester and B. Sakmann. Calcium dynamics associated with action potentials in single nerve terminals of pyramidal cells in layer $2 / 3$ of the young rat neocortex. $J$. Physiol., 529: 625-46, 2000. 
[115] S.B. Kombian, D. Mouginot, and Q.J. Pittman. Dendritically released peptides act as retrograde modulators of afferent excitation in the supraoptic nucleus in vitro. Neuron, 19: 903-12, 1997.

[116] S. Kondo and A. Marty. Protein kinase A-mediated enhancement of miniature IPSC frequency by noradrenaline in rat cerebellar stellate cells. J. Physiol., 498: 165-76, 1997.

[117] A. Konnerth, J. Dreessen, and G.J. Augustine. Brief dendritic calcium signals initiate long-lasting synaptic depression in cerebellar Purkinje cells. Proc. Natl. Acad. Sci. U.S.A., 89: 7051-5, 1992.

[118] A. Konnerth, U. Heinemann, and Y. Yaari. Nonsynaptic epileptogenesis in the mammalian hippocampus in vitro. i. Development of seizurelike activity in low extracellular calcium. J. Neurophysiol., 56: 409-23, 1986.

[119] L. Korbo, B.B. Andersen, O. Ladefoged, and A. Moller. Total numbers of various cell types in rat cerebellar cortex estimated using an unbiased stereological method. Brain Res., 609: 262-8, 1993.

[120] H. Korn and H. Axelrad. Electrical inhibition of Purkinje cells in the cerebellum of the rat. Proc. Natl. Acad. Sci. U.S.A., 77: 6244-7, 1980.

[121] H. Korn, A. Triller, and D.S. Faber. Structural correlates of recurrent collateral interneurons producing both electrical and chemical inhibitions of the Mauthner cell. Proc. $R$. Soc. Lond. B. Biol. Sci., 202: 533-8, 1978.

[122] M. Korte, P. Carroll, E. Wolf, G. Brem, H. Thoenen, and T. Bonhoeffer. Hippocampal Long-Term Potentiation is impaired in mice lacking brain- derived neurotrophic factor. Proc. Natl. Acad. Sci. U.S.A., 92: 8856-60, 1995.

[123] M. Korte, O. Griesbeck, C. Gravel, P. Carroll, V. Staiger, H. Thoenen, and T. Bonhoeffer. Virus-mediated gene transfer into hippocampal CA1 region restores Long-Term Potentiation in Brain-Derived Neurotrophic Factor mutant mice. Proc. Natl. Acad. Sci. U.S.A., 93: 12547-52, 1996. 
[124] G. Krapivinsky, L. Krapivinsky, K. Wickman, and D.E. Clapham. G beta gamma binds directly to the G protein-gated K+ channel, IK(ach). J. Biol. Chem., 270: 29059-62, 1995.

[125] A.C. Kreitzer, A.G. Carter, and W.G. Regehr. Inhibition of interneuron firing extends the spread of endocannabinoid signaling in the cerebellum. Neuron, 34: 787-96, 2002.

[126] A.C. Kreitzer and W.G. Regehr. Retrograde inhibition of presynaptic calcium influx by endogenous cannabinoids at excitatory synapses onto Purkinje cells. Neuron, 29: 71727, 2001a.

[127] A.C. Kreitzer and W.G. Regehr. Cerebellar Depolarization-induced Suppression of Inhibition is mediated by endogenous cannabinoids. J. Neurosci., 21: RC174, 2001 b.

[128] H. Kuromi and Y. Kidokoro. Tetanic stimulation recruits vesicles from reserve pool via a cAMP- mediated process in Drosophila synapses. Neuron, 27(1): 133-43, 2000.

[129] L.M. Larramendi. Electron microscopic studies of cerebellar interneurons. UCLA Forum Med. Sci., 11: 289-307, 1969.

[130] E.M. Lasater. Retinal horizontal cell gap junctional conductance is modulated by dopamine through a cyclic AMP-dependent protein kinase. Proc. Natl. Acad. Sci. U.S.A., 84: 7319-23, 1987.

[131] G. Laube, J. Roper, J.C. Pitt, S. Sewing, U. Kistner, C.C. Garner, O. Pongs, and R.W. Veh. Ultrastructural localization of Shaker-related potassium channel subunits and synapseassociated protein 90 to septate-like junctions in rat cerebellar pinceaux. Brain Res. Mol. Brain Res., 42: 51-61, 1996.

[132] R.A. Lenz, J.J. Wagner, and B.E. Alger. N- and L-type calcium channel involvement in depolarization-induced suppression of inhibition in rat hippocampal CA1 cells. $J$. Physiol., 512(1): 61-7, 1998.

[133] J. Lerma, A.S. Herranz, O. Herreras, V. Abraira, and R. Martin del Rio. In vivo determination of extracellular concentration of amino acids in the rat hippocampus. A method based on brain dialysis and computerized analysis. Brain Res., 384: 145-55, 1986. 
[134] C. Levenes, H. Daniel, and F. Crepel. Retrograde modulation of transmitter release by postsynaptic subtype 1 metabotropic glutamate receptors in the rat cerebellum. J. Physiol., 537(1): 125-40, 2001.

[135] C. Levenes, H. Daniel, P. Soubrie, and F. Crepel. Cannabinoids decrease excitatory synaptic transmission and impair Long-Term Depression in rat cerebellar Purkinje cells. J. Physiol., 510(3): 867-79, 1998.

[136] Z. Li and G.I. Hatton. Oscillatory bursting of phasically firing rat supraoptic neurones in low-Ca2+ medium: $\mathrm{Na}+$ influx, cytosolic $\mathrm{Ca} 2+$ and gap junctions. J. Physiol., 496: 379-94, 1996.

[137] I. Llano, R. DiPolo, and A. Marty. Calcium-induced calcium release in cerebellar Purkinje cells. Neuron, 12: 663-73, 1994.

[138] I. Llano and H.M. Gerschenfeld. Inhibitory synaptic currents in stellate cells of rat cerebellar slices. J. Physiol., 468: 177-200, 1993.

[139] I. Llano, J. Gonzalez, C. Caputo, F.A. Lai, L.M. Blayney, Y.P. Tan, and A. Marty. Presynaptic calcium stores underlie large-amplitude miniature IPSCs and spontaneous calcium transients. Nat. Neurosci., 3: 1256-65, 2000.

[140] I. Llano, N. Leresche, and A. Marty. Calcium entry increases the sensitivity of cerebellar Purkinje cells to applied GABA and decreases inhibitory synaptic currents. Neuron, 6: 565-74, 1991.

[141] C. Luscher, J. Streit, P. Lipp, and H.R. Luscher. Action potential propagation through embryonic dorsal root ganglion cells in culture. ii. Decrease of conduction reliability during repetitive stimulation. J. Neurophysiol., 72: 634-43, 1994.

[142] C. Luscher, J. Streit, R. Quadroni, and H.R. Luscher. Action potential propagation through embryonic dorsal root ganglion cells in culture. i. Influence of the cell morphology on propagation properties. J. Neurophysiol., 72: 622-33, 1994.

[143] E.R. Macagno, K. J. Muller, and R.M. Pitman. Conduction block silences parts of a chemical synapse in the leech central nervous system. J. Physiol., 387: 649-64, 1987. 
[144] G. Maccaferri and C.J. McBain. The hyperpolarization-activated current (Ih) and its contribution to pacemaker activity in rat CA1 hippocampal stratum oriens-alveus interneurones. J. Physiol., 497: 119-30, 1996.

[145] K. Mackie, W.A. Devane, and B. Hille. Anandamide, an endogenous cannabinoid, inhibits calcium currents as a partial agonist in N18 neuroblastoma cells. Mol. Pharmacol., 44: 498-503, 1993.

[146] K. Mackie and B. Hille. Cannabinoids inhibit N-type calcium channels in neuroblastomaglioma cells. Proc. Natl. Acad. Sci. U.S.A., 89: 3825-9, 1992.

[147] K. Mackie, Y. Lai, R. Westenbroek, and R. Mitchell. Cannabinoids activate an inwardly rectifying potassium conductance and inhibit Q-type calcium currents in ATT20 cells transfected with rat brain cannabinoid receptor. J. Neurosci., 15: 6552-61, 1995.

[148] T. Maejima, K. Hashimoto, T. Yoshida, A. Aiba, and M. Kano. Presynaptic inhibition caused by retrograde signal from metabotropic glutamate to cannabinoid receptors. $\mathrm{Neu}$ ron, 31(3): 463-75, 2001.

[149] F. Maingret, A.J. Patel, M. Lazdunski, and E. Honore. The endocannabinoid anandamide is a direct and selective blocker of the background $\mathrm{K}(+)$ channel TASK-1. Embo J., 20: 47-54, 2001.

[150] R. Malinow and R.C. Malenka. Ampa receptor trafficking and synaptic plasticity. Annu. Rev. Neurosci., 25: 103-26, 2002.

[151] R. Malinow and R.W. Tsien. Presynaptic enhancement shown by whole-cell recordings of Long-Term Potentiation in hippocampal slices. Nature, 346: 177-80, 1990.

[152] T. Manabe, D.J. Wyllie, D.J. Perkel, and R.A. Nicoll. Modulation of synaptic transmission and long-term potentiation: effects on paired pulse facilitation and EPSC variance in the CA1 region of the hippocampus. J. Neurophysiol., 70: 1451-9, 1993.

[153] P. Mann-Metzer and Y. Yarom. Electrotonic coupling interacts with intrinsic properties to generate synchronized activity in cerebellar networks of inhibitory interneurons. $J$. Neurosci., 19: 3298-306, 1999.

[154] O. Manzoni and J. Bockaert. Cannabinoids inhibit GABAergic synaptic transmission in mice nucleus accumbens. Eur. J. Pharmacol., 412: R3-5, 2001. 
[155] S. Marinelli, C.W. Vaughan, M.J. Christie and M. Connor. Capsaicin activation of glutamatergic synaptic transmission in the rat locus coeruleus in vitro. J. Physiol., 543: 531-40, 2002.

[156] G. Marsicano, C.T. Wotjak, S.C. Azad, T. Bisogno, G. Rammes, M.G. Cascio, H. Hermann, J. Tang, C. Hofmann, W. Zieglgansberger, V. Di Marzo, and B. Lutz. The endogenous cannabinoid system controls extinction of aversive memories. Nature, 418: 530-4, 2002.

[157] B. Martin, A. Bloom, A. Howlett, and S. Welch. Cannabinoid action in the central nervous system. NIDA Res. Monogr., 90: 275-83, 1988.

[158] A. Marty and I. Llano. Modulation of inhibitory synapses in the mammalian brain. Curr. Opin. Neurobiol., 5: 335-41, 1995.

[159] L.A. Matsuda, T.I. Bonner, and S.J. Lolait. Localization of cannabinoid receptor mRNA in rat brain. J. Comp. Neurol., 327: 535-50, 1993.

[160] L.A. Matsuda, S.J. Lolait, M.J. Brownstein, A. C. Young, and T.I. Bonner. Structure of a cannabinoid receptor and functional expression of the cloned cDNA. Nature, 346: $561-4,1990$.

[161] D.A. McCormick and H.C. Pape. Noradrenergic and serotonergic modulation of a hyperpolarization activated cation current in thalamic relay neurones. J. Physiol., 431: 319-42, 1990.

[162] R. Mechoulam, S. Ben-Shabat, L. Hanus, M. Ligumsky, N.E. Kaminski, A.R. Schatz, A. Gopher, S. Almog, B.R. Martin, D.R. Compton, and et al. Identification of an endogenous 2-monoglyceride, present in canine gut, that binds to cannabinoid receptors. Biochem. Pharmacol., 50: 83-90, 1995.

[163] A.H. Meyer, I. Katona, M. Blatow, A. Rozov, and H. Monyer. In vivo labeling of parvalbumin-positive interneurons and analysis of electrical coupling in identified neurons. J. Neurosci., 22: 7055-64, 2002.

[164] H.B. Michelson and R.K. Wong. Synchronization of inhibitory neurones in the guineapig hippocampus in vitro. J. Physiol., 477: 35-45, 1994. 
[165] J. Midtgaard, N. Lasser-Ross, and W.N. Ross. Spatial distribution of Ca2+ influx in turtle Purkinje cell dendrites in vitro: role of a transient outward current. J. Neurophysiol., 70: 2455-69, 1993.

[166] I.M. Mintz, B.L. Sabatini, and W.G. Regehr. Calcium control of transmitter release at a cerebellar synapse. Neuron, 15: 675-88, 1995.

[167] I.M. Mintz, V.J. Venema, K.M. Swiderek, T.D. Lee, B.P. Bean, and M.E. Adams. P-type calcium channels blocked by the spider toxin omega-AGA-IV. Nature, 355: 827-9, 1992.

[168] D.L. Misner and J.M. Sullivan. Mechanism of cannabinoid effects on Long-Term Potentiation and Depression in hippocampal ca1 neurons. J. Neurosci., 19: 6795-805, 1999.

[169] W. Morishita and B.E. Alger. Sr2+ supports depolarization-induced suppression of inhibition and provides new evidence for a presynaptic expression mechanism in rat hippocampal slices. J. Physiol., 505: 307-17, 1997.

[170] W. Morishita and B.E. Alger. Evidence for endogenous excitatory amino acids as mediators in DSI of GABA(a)ergic transmission in hippocampal CA1. J. Neurophysiol., 82: 2556-64, 1999.

[171] W. Morishita and B.E. Alger. Direct depolarization and antidromic action potentials transiently suppress dendritic IPSPs in hippocampal CA1 pyramidal cells. J. Neurophysiol., 85: 480-4, 2001.

[172] W. Morishita, S.A. Kirov, and B.E. Alger. Evidence for metabotropic glutamate receptor activation in the induction of Depolarization-induced Suppression of Inhibition in hippocampal ca1. J. Neurosci., 18: 4870-82, 1998.

[173] W. Morishita, S.A. Kirov, T.A. Pitler, L.A. Martin, R.A. Lenz, and B.E. Alger. Nethylmaleimide blocks Depolarization-induced Suppression of Inhibition and enhances GABA release in the rat hippocampal slice in vitro. J. Neurosci., 17: 941-50, 1997.

[174] V. Morisset and L. Urban. Cannabinoid-induced presynaptic inhibition of glutamatergic EPSCs in substantia gelatinosa neurons of the rat spinal cord. J. Neurophysiol., 86: 40-8, 2001.

[175] S. Munro, K.L. Thomas, and M. Abu-Shaar. Molecular characterization of a peripheral receptor for cannabinoids. Nature, 365: 61-5, 1993. 
[176] Z. Nusser, E. Mulvihill, P. Streit, and P. Somogyi. Subsynaptic segregation of metabotropic and ionotropic glutamate receptors as revealed by immunogold localization. Neuroscience, 61: 421-7, 1994.

[177] T. Ohno-Shosaku, T. Maejima, and M. Kano. Endogenous cannabinoids mediate retrograde signals from depolarized postsynaptic neurons to presynaptic terminals. Neuron, 29: 729-38, 2001.

[178] T. Ohno-Shosaku, S. Sawada, and C. Yamamoto. Properties of Depolarization-induced Suppression of Inhibitory transmission in cultured rat hippocampal neurons. Pflugers Arch., 435: 273-9, 1998.

[179] T. Ohno-Shosaku, J. Shosaku, H. Tsubokawa, and M. Kano. Cooperative endocannabinoid production by neuronal depolarization and group 1 metabotropic glutamate receptor activation. Eur. J. Neurosci., 15: 953-61, 2002.

[180] T. Ohno-Shosaku, H. Tsubokawa, I. Mizushima, N. Yoneda, A. Zimmer, and M. Kano. Presynaptic cannabinoid sensitivity is a major determinant of depolarization-induced retrograde suppression at hippocampal synapses. J. Neurosci., 22: 3864-72, 2002b.

[181] X. Pan, S.R. Ikeda, and D.L. Lewis. Rat brain cannabinoid receptor modulates N-type Ca2+ channels in a neuronal expression system. Mol. Pharmacol., 49: 707-14, 1996.

[182] V. Parpura and P.G. Haydon. Physiological astrocytic calcium levels stimulate glutamate release to modulate adjacent neurons. Proc. Natl. Acad. Sci. U.S.A., 97: 8629-34, 2000.

[183] A.E. Pereda and D.S. Faber. Activity-dependent short-term enhancement of intercellular coupling. J. Neurosci., 16: 983-92, 1996.

[184] D.A. Pettit, M.P. Harrison, J.M. Olson, R.F. Spencer, and G.A. Cabral. Immunohistochemical localization of the neural cannabinoid receptor in rat brain. J. Neurosci. Res., 51: 391-402, 1998.

[185] D. Piomelli, A. Giuffrida, A. Calignano, and F. Rodriguez de Fonseca. The endocannabinoid system as a target for therapeutic drugs. Trends Pharmacol. Sci., 21: 218-24, 2000.

[186] T.A. Pitler and B.E. Alger. Postsynaptic spike firing reduces synaptic GABAa responses in hippocampal pyramidal cells. J. Neurosci., 12: 4122-32, 1992. 
[187] T.A. Pitler and B.E. Alger. Depolarization-induced Suppression of GABAergic Inhibition in rat hippocampal pyramidal cells: $G$ protein involvement in a presynaptic mechanism. Neuron, 13: 1447-55, 1994.

[188] J.S. Poling, M.A. Rogawski, N. Salem, and S. Vicini. Anandamide, an endogenous cannabinoid, inhibits Shaker-related voltage-gated K+ channels. Neuropharmacology, 35: 983-91, 1996.

[189] C. Pouzat and S. Hestrin. Developmental regulation of basket/stellate cell to Purkinje cell synapses in the cerebellum. J. Neurosci., 17: 9104-12, 1997.

[190] C. Pouzat and A. Marty. Autaptic inhibitory currents recorded from interneurones in rat cerebellar slices. J. Physiol., 509: 777-83, 1998.

[191] C. Pouzat and A. Marty. Somatic recording of GABAergic autoreceptor current in cerebellar stellate and basket cells. J. Neurosci., 19(5): 1675-90, 1999.

[192] L. Prezeau, O. Manzoni, V. Homburger, F. Sladeczek, K. Curry, and J. Bockaert. Characterization of a metabotropic glutamate receptor: direct negative coupling to adenylyl cyclase and involvement of a pertussis toxin- sensitive G protein. Proc. Natl. Acad. Sci. U.S.A., 89: 8040-4, 1992.

[193] S. Ramon y Cajal. Histology of the Nervous System of Man and Vertebrates. English Translated Edition. Oxford University Press, New York, NY, USA, 1995.

[194] M.J. Rebecchi and S.N. Pentyala. Structure, function, and control of phosphoinositidespecific phospholipase C. Physiol. Rev., 80: 1291-335, 2000.

[195] E. Reuveny, P.A. Slesinger, J. Inglese, J.M. Morales, J.A. Iniguez-Lluhi, R.J. Lefkowitz, H.R. Bourne, Y.N. Jan, and L. Y. Jan. Activation of the cloned muscarinic potassium channel by G protein beta gamma subunits. Nature, 370: 143-6, 1994.

[196] C. Risinger and M.K. Bennett. Differential phosphorylation of syntaxin and synaptosome-associated protein of $25 \mathrm{kda}$ (snap-25) isoforms. J. Neurochem., 72: 61424, 1999.

[197] D. Robbe, G. Alonso, F. Duchamp, J. Bockaert, and O.J. Manzoni. Localization and mechanisms of action of cannabinoid receptors at the glutamatergic synapses of the mouse nucleus accumbens. J. Neurosci., 21: 109-16, 2001. 
[198] D. Robbe, M. Kopf, A. Remaury, J. Bockaert, and O.J. Manzoni. Endogenous cannabinoids mediate Long-Term synaptic Depression in the nucleus accumbens. Proc. Natl. Acad. Sci. U.S.A., 99: 8384-8, 2002.

[199] J.J. Rodriguez, K. Mackie and V.M. Pickel. Ultrastructural localization of the CB1 cannabinoid receptor in mu-opioid receptor patches of the rat Caudate putamen nucleus. J. Neurosci., 21: 823-33, 2001.

[200] B. Rorig, G. Klausa, and B. Sutor. Dye coupling between pyramidal neurons in developing rat prefrontal and frontal cortex is reduced by protein kinase A activation and dopamine. J. Neurosci., 15: 7386-400, 1995.

[201] B.L. Sabatini and W.G. Regehr. Control of neurotransmitter release by presynaptic waveform at the granule cell to Purkinje cell synapse. J. Neurosci., 17: 3425-35, 1997.

[202] F. Saitow and S. Konishi. Excitability increase induced by beta-adrenergic receptormediated activation of hyperpolarization-activated cation channels in rat cerebellar basket cells. J. Neurophysiol., 84: 2026-34, 2000.

[203] T. Sakaba and E. Neher. Preferential potentiation of fast-releasing synaptic vesicles by cAMP at the Calyx of Held. Proc. Natl. Acad. Sci. U.S.A., 98: 331-6, 2001.

[204] S. Satake, F. Saitow, J. Yamada, and S. Konishi. Synaptic activation of AMPA receptors inhibits GABA release from cerebellar interneurons. Nat. Neurosci., 3: 551-8, 2000.

[205] E. Schlicker and M. Kathmann. Modulation of transmitter release via presynaptic cannabinoid receptors. Trends Pharmacol. Sci., 22: 565-72, 2001.

[206] R. Schneggenburger and E. Neher. Intracellular calcium dependence of transmitter release rates at a fast central synapse. Nature, 406: 889-93, 2000.

[207] P. Schweitzer. Cannabinoids decrease the $K(+)$ M-current in hippocampal CA1 neurons. J. Neurosci., 20: 51-8, 2000.

[208] I. Segev. Computer study of presynaptic inhibition controlling the spread of action potentials into axonal terminals. J. Neurophysiol., 63: 987-98, 1990.

[209] G.M. Shepherd. The synaptic organization of the brain. Oxford University Press, New York, N.Y, U.S.A, 2001. 
[210] S.M. Sherman and R.W. Guillery. Exploring the Thalamus. Academic Press, San Diego, CA, U.S.A, 2001.

[211] S.A. Siegelbaum, J.S. Camardo, and E.R. Kandel. Serotonin and cyclic AMP close single K+ channels in Aplysia sensory neurones. Nature, 299: 413-7, 1982.

[212] D.M. Slipetz, G.P. O’Neill, L. Favreau, C. Dufresne, M. Gallant, Y. Gareau, D. Guay, M. Labelle, and K. M. Metters. Activation of the human peripheral cannabinoid receptor results in inhibition of Adenylyl Cyclase. Mol. Pharmacol., 48: 352-61, 1995.

[213] P. Somogyi and J. Hamori. A quantitative electron microscopic study of the Purkinje cell axon initial segment. Neuroscience, 1: 361-5, 1976.

[214] C. Sotelo and R. Llinas. Specialized membrane junctions between neurons in the vertebrate cerebellar cortex. J. Cell. Biol., 53: 271-89, 1972.

[215] A.P. Southan, N.P. Morris, G.J. Stephens, and B. Robertson. Hyperpolarization-activated currents in presynaptic terminals of mouse cerebellar basket cells. J. Physiol., 526: 91-7, 2000.

[216] A.P. Southan and B. Robertson. Electrophysiological characterization of voltage-gated $\mathrm{K}(+)$ currents in cerebellar basket and Purkinje cells: Kv1 and Kv3 channel subfamilies are present in basket cell nerve terminals. J. Neurosci., 20: 114-22, 2000.

[217] A.N. Spencer, J. Przysiezniak, J. Acosta-Urquidi, and T.A. Basarsky. Presynaptic spike broadening reduces junctional potential amplitude. Nature, 340: 636-8, 1989.

[218] N. Stella and D. Piomelli. Receptor-dependent formation of endogenous cannabinoids in cortical neurons. Eur. J. Pharmacol., 425: 189-96, 2001.

[219] N. Stella, P. Schweitzer, and D. Piomelli. A second endogenous cannabinoid that modulates Long-Term Potentiation. Nature, 388: 773-8, 1997.

[220] G.J. Stephens, N.P. Morris, R.E. Fyffe, and B. Robertson. The CaV2.1/alpha1a (P/Qtype) voltage-dependent calcium channel mediates inhibitory neurotransmission onto mouse cerebellar Purkinje cells. Eur J. Neurosci., 13: 1902-12, 2001. 
[221] J.M. Sullivan. Mechanisms of cannabinoid-receptor-mediated inhibition of synaptic transmission in cultured hippocampal pyramidal neurons. J. Neurophysiol., 82: 1286-94, 1999.

[222] K.R. Svoboda and C.R. Lupica. Opioid inhibition of hippocampal interneurons via modulation of potassium and hyperpolarization-activated cation (Ih) currents. J. Neurosci., 18: 7084-98, 1998.

[223] B. Szabo, L. Dorner, C. Pfreundtner, W. Norenberg, and K. Starke. Inhibition of GABAergic inhibitory postsynaptic currents by cannabinoids in rat corpus striatum. Neuroscience, 85: 395-403, 1998.

[224] B. Szabo, I. Wallmichrath, P. Mathonia, and C. Pfreundtner. Cannabinoids inhibit excitatory neurotransmission in the substantia nigra pars reticulata. Neuroscience, 97: 89-97, 2000.

[225] A. Szallasi and V. Di Marzo. New perspectives on enigmatic vanilloid receptors. Trends in Neurosci., 23: 491-7, 2000.

[226] K.A. Takahashi and D.J. Linden. Cannabinoid receptor modulation of synapses received by cerebellar Purkinje cells. J. Neurophysiol., 83: 1167-80, 2000.

[227] Y. Tamaru, S. Nomura, N. Mizuno, and R. Shigemoto. Distribution of metabotropic glutamate receptor mGluR3 in the mouse cns: differential location relative to pre- and postsynaptic sites. Neuroscience, 106: 481-503, 2001.

[228] G. Tamas, E.H. Buhl, A. Lorincz, and P. Somogyi. Proximally targeted GABAergic synapses and gap junctions synchronize cortical interneurons. Nat. Neurosci., 3: 366$71,2000$.

[229] W. Timmerman and B.H. Westerink. Brain microdialysis of GABA and glutamate: what does it signify? Synapse, 27: 242-61, 1997.

[230] R.D. Traub, N. Kopell, A. Bibbig, E.H. Buhl, F.E. LeBeau, and M.A. Whittington. Gap junctions between interneuron dendrites can enhance synchrony of gamma oscillations in distributed networks. J. Neurosci., 21: 9478-86, 2001. 
[231] K. Tsou, S. Brown, M.C. Sanudo-Pena, K. Mackie, and J.M. Walker. Immunohistochemical distribution of cannabinoid CB1 receptors in the rat central nervous system. Neuroscience, 83: 393-411, 1998.

[232] K. Tsou, K. Mackie, M.C. Sanudo-Pena, and J.M. Walker. Cannabinoid CB1 receptors are localized primarily on cholecystokinin- containing GABAergic interneurons in the rat hippocampal formation. Neuroscience, 93: 969-75, 1999.

[233] K. Tsou, M.I. Nogueron, S. Muthian, M.C. Sanudo-Pena, C.J. Hillard, D.G. Deutsch, and J.M. Walker. Fatty Acid Amide Hydrolase is located preferentially in large neurons in the rat central nervous system as revealed by immunohistochemistry. Neurosci. Lett., 254: 137-40, 1998.

[234] W. Twitchell, S. Brown, and K. Mackie Cannabinoids inhibit N- and P/Q-type calcium channels in cultured rat hippocampal neurons. J. Neurophysiol. 78 :43-50, 1997.

[235] N. Varma, G.C. Carlson, C. Ledent, and B.E. Alger. Metabotropic glutamate receptors drive the endocannabinoid system in hippocampus. J. Neurosci., 21: RC188, 2001.

[236] C.W. Vaughan, M. Connor, E.E. Bagley, and M.J. Christie. Actions of cannabinoids on membrane properties and synaptic transmission in rat periaqueductal gray neurons in vitro. Mol. Pharmacol., 57: 288-95, 2000.

[237] C.W. Vaughan, S.L. Ingram, M.A. Connor, and M.J. Christie. How opioids inhibit GABA-mediated neurotransmission. Nature, 390: 611-4, 1997.

[238] C.W. Vaughan, I.S. McGregor, and M.J. Christie. Cannabinoid receptor activation inhibits GABAergic neurotransmission in rostral ventromedial medulla neurons in vitro. Br. J. Pharmacol., 127: 935-40, 1999.

[239] J. L. Velazquez, D. Han, and P.L. Carlen. Neurotransmitter modulation of gap junctional communication in the rat hippocampus. Eur J. Neurosci., 9: 2522-31, 1997.

[240] L. Venance, D. Piomelli, J. Glowinski, and C. Giaume. Inhibition by anandamide of gap junctions and intercellular calcium signalling in striatal astrocytes. Nature, 376: 590-4, 1995. 
[241] P. Vincent, C.M. Armstrong, and A. Marty. Inhibitory synaptic currents in rat cerebellar Purkinje cells: modulation by postsynaptic depolarization. J. Physiol., 456: 453-71, 1992.

[242] P. Vincent and A. Marty. Neighboring cerebellar Purkinje cells communicate via retrograde inhibition of common presynaptic interneurons. Neuron, 11: 885-93, 1993.

[243] P. Vincent and A. Marty. Fluctuations of inhibitory postsynaptic currents in Purkinje cells from rat cerebellar slices. J. Physiol., 494: 183-99, 1996.

[244] A. Vyshedskiy, T. Allana, and J.W. Lin. Analysis of presynaptic Ca2+ influx and transmitter release kinetics during facilitation at the inhibitor of the crayfish neuromuscular junction. J. Neurosci., 20: 6326-32, 2000.

[245] J.J. Wagner and B.E. Alger. Increased neuronal excitability during depolarizationinduced suppression of inhibition in rat hippocampus. J. Physiol., 495: 107-12, 1996.

[246] J. Wang and R.S. Zucker. Photolysis-induced suppression of inhibition in rat hippocampal ca1 pyramidal neurons. J. Physiol., 533: 757-63, 2001.

[247] S.S. Wang, W. Denk, and M. Hausser. Coincidence detection in single dendritic spines mediated by calcium release. Nat. Neurosci., 3: 1266-73, 2000.

[248] T.W. White, D.L. Paul. Genetic diseases and gene knockouts reveal diverse connexin functions. Annu. Rev. Physiol., 61: 283-310, 1999.

[249] M.A. Whittington, R.D. Traub, and J.G. Jefferys. Synchronized oscillations in interneuron networks driven by metabotropic glutamate receptor activation. Nature, 373: 612-5, 1995.

[250] J.H. Williams, M.L. Errington, M.A. Lynch, and T.V. Bliss. Arachidonic acid induces a long-term activity-dependent enhancement of synaptic transmission in the hippocampus. Nature, 341: 739-42, 1989.

[251] R.I. Wilson, G. Kunos, and R.A. Nicoll. Presynaptic specificity of endocannabinoid signaling in the hippocampus. Neuron, 31: 453-62, 2001.

[252] R.I. Wilson and R.A. Nicoll. Endogenous cannabinoids mediate retrograde signalling at hippocampal synapses. Nature, 410: 588-92, 2001. 
[253] Y. Yamakawa and T. Hirano. Contribution of mGluR1 to the basal activity of a mouse cerebellar Purkinje neuron. Neurosci. Lett., 277: 103-6, 1999.

[254] T. Yoshida, K. Hashimoto, A. Zimmer, T. Maejima, K. Araishi, and M. Kano. The cannabinoid CB1 receptor mediates retrograde signals for Depolarization-induced Suppression of Inhibition in cerebellar Purkinje cells. J. Neurosci., 22: 1690-7, 2002.

[255] M. Yoshihara, K. Suzuki, and Y. Kidokoro. Two independent pathways mediated by cAMP and protein kinase A enhance spontaneous transmitter release at Drosophila neuromuscular junctions. J. Neurosci., 20: 8315-22, 2000.

[256] J.F. Zhang, P.T. Ellinor, R.W. Aldrich, and R.W. Tsien. Multiple structural elements in voltage-dependent $\mathrm{Ca} 2+$ channels support their inhibition by $\mathrm{G}$ proteins. Neuron, 17: 991-1003, 1996.

[257] Y. Zilberter, K.M. Kaiser and B. Sakmann. Dendritic GABA release depresses excitatory transmission between layer $2 / 3$ pyramidal and bitufted neurons in rat neocortex. Neuron, 24: 979-88, 1999.

[258] Y. Zilberter. Dendritic release of glutamate suppresses synaptic inhibition of pyramidal neurons in rat neocortex. J. Physiol., 528: 489-496, 2000.

[259] P.M. Zygmunt, J. Petersson, D.A. Andersson, H. Chuang, M. Sorgard, V. Di Marzo, D. Julius, and E.D. Hogestatt. Vanilloid receptors on sensory nerves mediate the vasodilator action of anandamide. Nature, 400: 452-7, 1999. 


\section{Marco Alberto Diana}

\section{Lebenslauf}

25.01.1969

1975-1980

1980-1983

1983-1988

Juli 1988

1989

1993-1995

31.03.1995

1995-1996

1996-1998

1998-2001

2001-2002
Geboren in Mailand (Italien).

Besuch der Grundschule in Mailand.

Besuch der Mittelschule in Mailand.

Besuch des staatlichen Gymnasiums - wissentschaftlicher Zweig - Liceo Scientifico "Luigi Cremona" in Mailand.

Abitur am Gymnasium "Luigi Cremona" (60/60).

Immatrikulation an der Università degli Studi di Milano in Mailand für das Fach Physik.

Diplomarbeit am Istituto Scientifico Dibit, Ospedale San Raffaele in Mailand in der Abteilung von Neurobiologie von Professor Jacopo Meldolesi und unter der Anleitung von Dr. Antonio Malgaroli.

Diplom im Fach Physik (110/110 cum Laude).

Sozialdienstpflicht.

Forschungsarbeit am Dibit Institut in Mailand im Labor von Dr. Malgaroli. Doktorarbeit am Max-Planck-Institut fuer biophysikalische Chemie in der Abteilung Zelluläre Neurobiologie unter der Anleitung von Dr. Alain Marty und der Betreuung von Professor Erwin Neher seitens der Universität Göttingen.

Nach dem Umzug der Abteilung von Göttingen nach Paris wird die Doktorarbeit unter der Anleitung von Dr. Marty im Laboratoire de Physiologie Cérébrale an der Universität René Descartes-Paris 5 abgeschlossen. 